\title{
EXTENDING THE SENSITIVITY TO THE DETECTION OF WIMP DARK MATTER WITH AN IMPROVED UNDERSTANDING OF THE LIMITING NEUTRON BACKGROUNDS
}

\author{
by \\ SHARMILA KAMAT
}

Submitted in partial fulfilment of the requirements

For the degree of Doctor of Philosophy

Dissertation Advisor: Dr Daniel S. Akerib

Department of Physics

Case Western Reserve University

January 2005 


\section{CASE WESTERN RESERVE UNIVERSITY SCHOOL OF GRADUATE STUDIES}

We hereby approve the dissertation of

candidate for the Ph.D. degree.

(signed)

Daniel S. Akerib, Chair of the Committee

Tanmay Vachaspati

Corbin E. Covault

J. Christopher Mihos

(date) 
TO MY PARENTS 


\section{Contents}

1 Introduction $\quad 19$

1.1 The Dark Side of the Universe . . . . . . . . . . . . . . . . . . 19

1.2 Searching for WIMPs . . . . . . . . . . . . . . . 22

1.3 Layout of the thesis . . . . . . . . . . . . . . 25

2 The Dark Matter Problem 28

2.1 The State of the Universe . . . . . . . . . . . . . 28

2.2 A Flat Universe . . . . . . . . . . . . . . . . . 29

2.2.1 Cosmic Microwave Background Radiation . . . . . . . . . 31

2.3 A Dark Universe . . . . . . . . . . . . . . . . . 32

2.3.1 The First Evidence . . . . . . . . . . . . . . 33

2.3.2 Rotational Curves of Spiral Galaxies . . . . . . . . . . . 34

2.3.3 Elliptical galaxies .................. 35

2.3.4 Clusters of galaxies . . . . . . . . . . . . . 36

2.4 Cold Exotic Dark Matter . . . . . . . . . . . . . . . . . 39

2.4.1 Big Bang Nucleosynthesis . . . . . . . . . . . . . 39

2.4.2 Cold and hot dark matter ............ . . 41 
2.5 WIMP Dark Matter . . . . . . . . . . . . . . . 42

2.5.1 WIMP relic density . . . . . . . . . . . . . . . 42

2.5.2 The Neutralino as a Dark Matter Candidate . . . . . . . . 47

2.6 WIMP detection $\ldots \ldots \ldots \ldots \ldots \ldots$

2.6.1 Direct detection . . . . . . . . . . . . . . . . 48

2.6 .2 Indirect detection . . . . . . . . . . . . . . . 53

2.7 The CDMS approach . . . . . . . . . . . . . . . 54

3 The CDMS Search for Dark Matter 59

3.1 The ZIP detector $\ldots \ldots \ldots \ldots \ldots \ldots$

3.1.1 Event-by-event discrimination . . . . . . . . . . . 60

3.1 .2 Two-fold signature $\ldots \ldots \ldots$. . . . . . . . . . . 61

3.1.3 The Charge Channel . . . . . . . . . . . . . . . . . . . 63

3.1.4 The Phonon Channel . . . . . . . . . . . . . . . . . . . . 65

3.1.5 Rise Time as a discrimination parameter . . . . . . . . . 68

3.2 Run 21 at Stanford . . . . . . . . . . . . . . . . . . . 70

3.2 .1 Rationale for the Run . . . . . . . . . . . . . . 70

3.2.2 Time line and structure of the Run . . . . . . . . . . 70

3.2.3 The Icebox and Dilution Refrigerator . . . . . . . . . . . 71

$3.3 \quad$ Backgrounds seen at SUF . . . . . . . . . . . . 71

3.4 First analysis of the $3 \mathrm{~V}$ data $\ldots \ldots \ldots \ldots \ldots$

3.4.1 Data cuts applied- . . . . . . . . . . . . . . . . 74

3.5 Maximum Likelihood Ratio Analysis of the extended Run 21 data . 76 


\section{The Neutron Background and how it Limits Sensitivity to WIMP $\begin{array}{ll}\text { Detection } & 81\end{array}$}

4.1 Sources of the Neutron Backgrounds . . . . . . . . . . . . . 82

4.1.1 Depth Dependence . . . . . . . . . . . . . . . . . . . 83

4.1.2 Neutrons from Natural Radioactivity of the Rock . . . . . 86

4.1.3 Neutrons from Radioactivity in the Shield . . . . . . . . . 86

4.2 Neutrons from Cosmic Ray Muons _. . . . . . . . . . . . . 87

4.2 .1 Mean Muon Energy . . . . . . . . . . . . . . . . . . . . 88

4.2 .2 Muon Capture by Nuclei . . . . . . . . . . . . . . . . . . 90

4.2.3 Neutrons from Electromagnetic Showers _. . . . . . . . 90

4.2.4 Muon-nucleus Inelastic Scattering . . . . . . . . . . . . . . 91

4.3 Uncertainties in the Neutron Yield . . . . . . . . . . . . . 93

4.4 The Neutron Flux at SUF . . . . . . . . . . . . . . . . 95

4.4.1 Muon-Induced Neutrons in the Shield . . . . . . . . . . . . 96

4.4.2 The Limiting Background at Stanford . . . . . . . . . . . 97

4.5 The Flux at Soudan . . . . . . . . . . . . . . . . . . . 98

4.5.1 Neutrons from the Shield . . . . . . . . . . . . . . . . . . 98

4.5.2 Contribution from the Rock _. . . . . . . . . . 99

4.5.3 Limiting Sensitivity . . . . . . . . . . . . . . . . . . . . 101

5 Simulating Neutron Backgrounds at a Shallow Site 105

5.1 Modelling the Neutrons . . . . . . . . . . . . . . . . . . 106

5.1.1 The GEANT Particle Transport Code . . . . . . . . . . . 106

5.1 .2 Coding in the Geometry . . . . . . . . . . . . . 108 
5.2 The Neutron Calibrations _. . . . . . . . . . . . . . . 110

5.3 Simulating the Calibrations . . . . . . . . . . . . . 111

5.4 3V Neutron Calibrations . . . . . . . . . . . . . . . . . . . 114

5.4.1 First analysis using the flat rise time cut . . . . . . . . 114

5.4 .2 Maximum Likelihood Analysis . . . . . . . . . . . . . 120

5.5 Muon coincident nuclear-recoil events . . . . . . . . . . . . 127

5.6 Simulating the Veto-coincident Neutrons . . . . . . . . . . . 129

5.6.1 First analysis using the flat rise time cut . . . . . . . 132

5.6 .2 Maximum Likelihood Analysis . . . . . . . . . . . 136

5.7 Use of the simulations . . . . . . . . . . . . . . . . 139

6 Improved WIMP Limits at the Shallow Site 144

6.1 Simulating the limiting neutron background $\ldots \ldots \ldots \ldots$

6.1.1 Using the Run 19 results to normalize the external neutron flux . . . . . . . . . . . . . . . . . . 148

6.1.2 Addition of an inner polyethylene layer . . . . . . . . . . 149

6.2 Veto Anti-coincident Data at $3 \mathrm{~V}$ charge bias . . . . . . . . . 150

6.2.1 Efficiency of the fiducial-volume cut . . . . . . . . . 150

6.2 .2 Total efficiency . . . . . . . . . . . . . . . . . . 154

6.2.3 Results of the first analysis of the 3V WIMP-search data . . 155

6.3 Consistency of the Neutron hypothesis . . . . . . . . . . 156

6.3 .1 3V WIMP-search data . . . . . . . . . . . . . 156

6.3.2 Predictions of the external neutron simulations $\ldots \ldots . .156$

6.3 .3 Testing the neutron hypothesis $\ldots \ldots \ldots$ 
6.3 .4 Improved Limits . . . . . . . . . . . . . . . . . 163

6.4 Using Maximum Likelihood Analysis Techniques for beta rejection . 165

6.4.1 Estimating the efficiencies of the data cuts . . . . . . 167

6.4.2 Estimating the neutron background . . . . . . . . . . 176

6.4.3 Limits on the WIMP-nucleon cross section . . . . . . . . . 176

7 The WIMP-search at a deeper site 182

7.1 Run 118 at the Soudan Underground Facility . . . . . . . . . . 182

7.1.1 Backgrounds seen at Soudan . . . . . . . . . . . . . . 183

7.1.2 Shielding the detectors . . . . . . . . . . . . . . . 184

7.1.3 The Detecting Assembly . . . . . . . . . . . . . . . . 185

7.1.4 Cryogenics and Data Acquisition . . . . . . . . . . 185

7.1.5 Time line of the Run . . . . . . . . . . . . . . . . . . . 189

7.1.6 Neutralizing the detectors . . . . . . . . . . . . . . . . 189

7.1.7 Calibration runs with the ${ }^{133} \mathrm{Ba}$ source . . . . . . . . . . . 189

7.1 .8 Event discrimination . . . . . . . . . . . . . . . . . . . 191

7.2 Defining the fiducial-volume cut . . . . . . . . . . . . . 193

7.3 Neutron calibrations with the ${ }^{252} \mathrm{Cf}$ source . . . . . . . . . . 197

7.3.1 Coding in the geometry _ . . . . . . . . . . . 197

7.3.2 Identifying optimal source positions . . . . . . . . . . . 198

7.3 .3 Defining the signal region . . . . . . . . . . . . 200

7.3 .4 Data cuts . . . . . . . . . . . . . . . . . 201

7.4 Analysis of data from the first Soudan WIMP-search run . . . . . . 207

7.4 .1 WIMP candidate events . . . . . . . . . . . . 208 
7.4.2 The unblinded analysis . . . . . . . . . . . . . . . 210

7.5 Limits on the WIMP-nucleon cross section . . . . . . . . . . 210

8 Improved Sensitivity at a Deeper site $\quad 216$

8.1 The Neutron Flux at Soudan . . . . . . . . . . . . . 217

8.1.1 Neutron production from muons at deep sites . . . . . . 218

8.2 Neutrons produced in the shield . . . . . . . . . . . . . 223

8.3 Neutrons produced in the rock . . . . . . . . . . . . . . 227

8.4 Simulating the vetoed neutron population $\ldots \ldots \ldots$

8.5 Analysis of veto-coincident nuclear-recoil events in the data . . . . 232

8.5.1 Veto coincident neutrons - comparing data and simulations. 235

8.6 Simulating the un-vetoed neutron population . . . . . . . . 236

8.7 Analysis of veto-anti-coincident nuclear-recoil events in the data . . 239

8.7.1 Veto anti-coincident multiple-scatter events . . . . . . . . 240

8.7.2 Veto-anti-coincident single-scatter nuclear-recoil events . . . 241

8.8 Predicting the neutron backgrounds . . . . . . . . . . . . . . 242

9 Towards a Detection $\quad 247$ 


\section{List of Figures}

2.1 Rotation curve of the spiral galaxy M33 . . . . . . . . . . 35

2.2 Gravitational lens . . . . . . . . . . . . . . . . . . 38

2.3 Evolution of abundance of light elements . . . . . . . . . . . . . . . 40

2.4 WIMP number density . . . . . . . . . . . . . . 46

2.5 Feynman diagrams of neutralino-nucleus interactions . . . . . . 51

3.1 ZIP detector ........................ 60

3.2 Charge and phonon measurement . . . . . . . . . . . 63

3.3 Rise time as a discrimination parameter. . . . . . . . . . . . 69

3.4 Icebox and shield at SUF. . . . . . . . . . . . . . 73

4.1 Neutron Backgrounds . . . . . . . . . . . . . . . . . . . . 83

4.2 Neutron flux as a function of depth . . . . . . . . . . . . 84

4.3 Neutron interaction rates in Silicon and Germanium . . . . . . . . . 85

4.4 Mean muon energy as a function of depth. . . . . . . . . . . . . 89

4.5 Muon spallation . . . . . . . . . . . . . . . . . . 91

5.1 Modelling the shield and icebox . . . . . . . . . . . . . . 109

5.2 Close-up of the tower in the icebox . . . . . . . . . . . . 110 
5.3 Neutron calibration source positions . . . . . . . . . . . . . . 112

5.4 Yield vs. Recoil for the 3V Neutron Calibration data. . . . . . . . 117

5.5 Data vs. MC: 3V Neutron calibration (all and multiple scatter events)118

5.6 Data vs. MC : Single scatter events in 3V Neutron Calibration . . . 119

5.7 Likelihood function ratio for neutron events. . . . . . . . . . . 123

5.8 6V Neutron Calibration - Comparison of Spectra . . . . . . . 127

5.9 3V Muon veto-coincident nuclear recoil events. . . . . . . . . . . 130

5.10 3V Muon-coincident data . . . . . . . . . . . . . . . . . 134

5.11 3V Muon-coincident single scatters . . . . . . . . . . . . 135

5.12 3V Veto Coincident Neutrons . . . . . . . . . . . . . . . 138

5.13 Single scatters in 6V Muon-coincident data. . . . . . . . . . . 140

6.1 Veto AntiCoincident Data taken at $3 \mathrm{~V}$ bias . . . . . . . . . . . 151

6.2 Qinner cut efficiencies: Data vs. MC . . . . . . . . . . . 153

6.3 Single scatters: Total efficiency. . . . . . . . . . . . . . . . . . 154

6.4 Efficiency for single scatter events in the Germanium detectors. . . 155

6.5 Yield plots: Nearest neighbor scatters . . . . . . . . . . . . 157

6.6 Comparison of spectra : External neutrons . . . . . . . . . . 160

6.7 K-S Test for germanium single scatter events . . . . . . . . . . . 161

6.8 K-S test of the Veto anti-coincident data and the internal and external neutron simulations. . . . . . . . . . . . . . 162

6.9 Limits on the WIMP-nucleon cross section. . . . . . . . . . . . . 164

6.10 K-S test for 3V calibration singles using the Likelihood Analysis. . . 168

6.11 Efficiency Comparison : 3V calibration . . . . . . . . . 170 
6.12 K-S test of single scatter nuclear recoil energy spectrum for 3V veto coincident neutrons. . . . . . . . . . . . . . . . 171

6.13 Efficiency comparisons: 3V internals . . . . . . . . . . . . . 172

6.14 K-S test for the single scatter nuclear recoil candidates for the $6 \mathrm{~V}$ Neutron calibrations. . . . . . . . . . . . . . . . . 173

6.15 Efficiency vs. time: 6V Neutron calibrations. . . . . . . . . . . 174

6.16 K-S test for the veto coincident single scatter nuclear recoil candidates at $6 \mathrm{~V}$ charge bias. . . . . . . . . . . . . . . 175

6.17 Comparison of efficiencies: 6V Internals . . . . . . . . . . . . 175

6.18 Limits on the WIMP-nucleon cross section (assuming background leakage). . . . . . . . . . . . . . . . . . . 179

6.19 Limits on the WIMP-nucleon cross section (assuming zero background leakage) . . . . . . . . . . . . . . . . 180

7.1 Icebox and shield at Soudan . . . . . . . . . . . . . . 186

7.2 Icebox and shield: Sketch of the layout. . . . . . . . . . . 187

7.3 Efficiency of Live Time . . . . . . . . . . . . . . . . . 190

7.4 Data and simulations comparison for the neutron calibration run for Run $118 \ldots \ldots \ldots \ldots$. . . . . . . . . . . . . . . 191

7.5 Ba calibration -comparisons . . . . . . . . . . . . . . . . . 192

7.6 The fiducial volume cut . . . . . . . . . . . . . . . . . 195

7.7 Efficiency of the fiducial volume cut from the neutron calibration data.196

7.8 Soudan Icebox simulation $\ldots \ldots \ldots$

7.9 Close-up of the two towers . . . . . . . . . . . . . . 200

7.10 Event rate vs Neutron source position . . . . . . . . . . . . . . 201

7.11 Yield vs. Recoil: Neutron calibrations . . . . . . . . . . . . . . 202 
7.12 Run 118 Neutron calibration: Data vs. MC . . . . . . . . . 205

7.13 Run 118 Neutron calibration: single scatters events for data and MC. 206

7.14 Veto AntiCoincident Single Scatter Nuclear Recoils _. . . . . . . 209

7.15 Event passing all cuts in unblinded analysis. . . . . . . . . . . . 211

7.16 Run 118 limits . . . . . . . . . . . . . . . . . . . . . 213

8.1 Neutron Yield vs. Depth . . . . . . . . . . . . . . . . . . 219

8.2 Mean muon energy vs. depth . . . . . . . . . . . . . 220

8.3 Veto-coincident events with no rise time cut applied. . . . . . . . . 233

8.4 Veto-coincident events- rise time cut . . . . . . . . . . . . 234

8.5 External Neutron Flux . . . . . . . . . . . . . . . . . . 237

8.6 External recoil spectra . . . . . . . . . . . . . . . . 238

8.7 Veto anti-coincidence events, rise time cut applied. . . . . . . . . 240

8.8 Veto anti-coincident singles, rise time cut applied. . . . . . . . . . 243 


\section{List of Tables}

4.1 Soudan rock composition . . . . . . . . . . . . . . . 87

5.1 Event rates for the neutron calibration: Data vs. Simulations _. . 120

5.2 Neutron calibration: Data vs Simulations (All events) . . . . . . . 121

5.3 Neutron calibration using the Likelihood cuts: Rates Comparison . 124

5.4 Neutron calibration: Data vs Simulations (All events) . . . . . . 125

5.5 Neutron calibration using the Likelihood cuts: Rates Comparison . 128

5.6 Neutron calibration: Data vs Simulations (All events) . . . . . . 128

5.7 Event rates for the neutron calibration: Data vs. Simulations . . 137

5.8 Neutron calibration using the Likelihood cuts: Rates Comparison . 138

5.9 Neutron calibration: Data vs Simulations (All events) . . . . . . . 139

5.10 Neutron calibration using the Likelihood cuts: Rates Comparison 139

5.11 Neutron calibration: Data vs Simulations (All events) . . . . . . 140

6.1 Neutron calibration using the Likelihood cuts: Rates Comparison . 158

7.1 Neutron calibration: Data vs Simulations (All events) . . . . . . 208

8.1 Expected rates-internals $\ldots \ldots \ldots \ldots \ldots \ldots$ 
8.2 Expected neutrons-internals . . . . . . . . . . . . . . 231

8.3 Veto coincident rates II . . . . . . . . . . . . . . . . 235

8.4 Expected rates-internals . . . . . . . . . . . . . . . 239 


\section{ACKNOWLEDGMents}

When I came to the USA from India five years ago with the intention of studying for a Ph.D. degree, I had been prepared for change. What amazed me, and still does till today, is the extent and scale of this change. For one, the bitter cold of a Cleveland winter could not have been farther removed from the sunny climes of my homeland, Goa. Unlike the weather back home, where the temperature moved up and down a few degrees throughout the year, here a typical Cleveland day would begin with bright sunshine and end with a snowstorm, with a light drizzle thrown in for good measure. As for the pace of life, it was as if I had suddenly stepped on the gas on a highway with no speed limits and populated with comatose highway patrols.

The first year of graduate school, with its six courses, Teaching Assistantship duties and qualifier exams, passed in a daze of long days, sleepless nights and a frantic effort to make each second stretch to a minute and each minute to an hour. Light switches that turn on the other way, cars driving on the wrong side of the road, people using the word like as a comma instead of a verb -everything was different! I discovered the virtues of coffee not as a beverage but as a sleepsuppressant.

And I learnt that you can travel half-way round the globe to find people who are as generous with their time and knowledge as family and friends back home.

Getting a Ph.D. degree is a long-winded journey. For me, the travails of commuting down that road were eased by the support generously extended by a large number of people. One of them has been my thesis advisor, Dan Akerib. I first had the opportunity to interact with Dan during my Teaching Assistantship in my first year. He was always willing to help, whether in assisting foreign students like me navigate the tricky waters of teaching in the United States or lending a sympathetic ear to freshmen agonizing over how to do error analysis as part of the Introductory Labs. In the four years that I was his Ph.D. student, I came to 
appreciate how fortunate I was to have him as my advisor. He takes his duties as a mentor very seriously. In the course of my association with him I have drawn freely on his help, be it with regard to his generosity with his time, his ability to cast aside the technicalities and 'tell a story' when it comes to presentations or his enormous reserves of patience (which I must confess I have sometimes tested to the full). He has always been receptive to individual interests - he did not hesitate to let me take three months off my research work to take up a national science writing fellowship and encouraged to continue pursuing my creative writing along with my research. I have sought his help in finding my niche in the experiment, when stuck with a simulation problem, when poring over literature on neutron backgrounds, before giving a seminar talk, all through the course of my job search and in the crunch time of thesis writing and defense preparation - and never returned empty-handed. Thank you, Dan, for your unwavering faith in my abilities.

I would like to express my thanks to Richard Schnee, the analysis pointsperson of our collaboration, for teaching me how to turn raw data into a physics result. His ability to think logically, to interpret graphs just by glancing at them and his strong command on statistical techniques coupled with his ever-willingness to share this knowledge with others, has stood me well during the last few years of my graduate studies.

To Thushara Perera, a graduate student and later post-doc in the group, I would like to say thanks for teaching me how to do Monte Carlo simulations and lending a helping hand in debugging particularly stubborn pieces of code. His enthusiasm in the project, and his desire to pass this on the newcomers in the group, helped me get a firm grounding in the experiment.

I'd like to thank fellow graduate students Don Driscoll for helping out with my computer woes whenever my Windows machine decided to act up (which I have to admit was quite often) and Gensheng Wang for being able to appreciate my feelings after a particularly tough Soudan shift. I am grateful to Mike Dragowsky for reading through my thesis chapters. Thanks to our technician Adam and the undergraduates David, Bryan and Brandon for their help with computer issues. I 
wish Darren Grant, Paul Brusov, Raul Hennings-Yeomans and Cathy Bailey well as they continue the search for WIMPs.

To Lawrence Krauss, our department Chair, a big thank you for his continuous support and appreciation of my interest in freelance writing. It was sheer luck for me to seek to do research in a department whose Chair not only did not look askance when I looked beyond the laboratory but actually encouraged me each step of the way. He encouraged me to apply for the American Association for Advancement of Science Mass Media Science and Engineering Fellowship which brought together two of my interests - scientific research and popular writing.

I would like to thank Charles Rosenblatt, Director of Graduate Studies, for his prompt help with academic and administrative issues. My defense committee - Tanmay Vachaspati, Corbin Covault and Chris Mihos - thank you for patiently bearing with my changes in schedule and for making the oral defense such a pleasant experience. I also thank our department secretaries - Lucy and Lori - for help with administrative (and mailing) issues.

I would like to thank Bernard Sadoulet, Spokesperson for the CDMS Collaboration, for his support during the course of my association with the experiment and in my job search. Blas Cabrera, thanks for ensuring that Big Blue behaved and gave me data for my thesis. Dan Bauer, thanks for patiently bearing with my many changes to the Soudan schedule. My fellow Soudan troupers - Vuk, Clarence, Walter, Joel, Long, Angela, Rupak, Mike, Jip, Paul and many others thanks for making the Soudan shifts a pleasant experience. Thanks Steve Yellin for help with the neutron simulations. I would like to thank everyone in the Collaboration for the support extended to me both at Soudan and at my home institution. The success at Soudan, with the current best-ever limits on dark matter detection could not have come to a more decent bunch of people.

I would like to thank my well-wishers - the late Prof. Kashinath Mahale for his continued encouragement, Dr. Vamona and Violante Sinari for showing a keen interest in my endeavors, Sushila and Arun Rao for being my cheerleaders and 
Datta and Lata Naik for their support. I would like to thank my friends - Laxmi and Tony, Sushmita and Verdi, Tim, Cheshana, Anjali, Carrie, Vivek, Nitin for their friendship and support during the last five years.

Lastly I would like to thank my family for their unstinted support in all my endeavors - my brother, Prashant, for patiently listening to my woes when the Ph.D. seemed too long a haul for me to navigate and my sister, Pratima, who provided the much-needed support as the defense date loomed near. To my parents, a heartfelt thanks for their unconditional love and belief in my capabilities. I would like to thank my father for refusing to accept that his daughter could be anything but an achiever and my mother for encouraging me to take up a career in astrophysics. I am glad that the date I chose for my thesis defense also happened to be my mother's birthday.

Thanks, everyone! 


\title{
Extending the Sensitivity to the Detection of WIMP Dark Matter with an Improved Understanding of the Limiting Neutron Backgrounds
}

\author{
Abstract \\ by \\ Sharmila Kamat
}

The Cryogenic Dark Matter Search (CDMS) uses position-sensitive Germanium and Silicon crystals in the direct detection of Weakly Interacting Massive Particles (WIMPs) believed to constitute most of the dark matter in the Universe. WIMP interactions with matter being rare, identifying and eliminating known backgrounds is critical for detection. Event-by-event discrimination by the detectors rejects the predominant gamma and beta backgrounds while Monte Carlo simulations help estimate, and subtract, the contribution from the neutrons.

This thesis describes the effort to understand neutron backgrounds as seen in the two stages of the CDMS search for WIMPs. The first stage of the experiment was at a shallow site at the Stanford Underground Facility where the limiting background came from high-energy neutrons produced by cosmic-ray muon interactions in the rock surrounding the cavern.

Simulations of this background helped inform the analysis of data from an experimental run at this site and served as input for the background reduction techniques necessary to set new exclusion limits on the WIMP-nucleon cross-section, excluding new parameter space for WIMPs of masses $8-20 \mathrm{GeV} / \mathrm{c}^{2}$.

This thesis considers the simulation methods used as well as how various event populations in the data served as checks on the simulations to allow them to be used in the interpretation of the WIMP-search data. The studies also confirmed the presence of a limiting neutron background at the shallow site, necessitating the move to the 713-meter deep Soudan Underground Facility. 
Similar computer-based studies helped quantify the neutron background seen at the deeper site and informed the analysis of the data emerging from the first physics run of the experiment at Soudan. In conjunction with the WIMP-search and calibration data, the simulations confirmed that increased depth considerably reduced the neutron backgrounds seen, greatly improving the sensitivity to WIMP detection.

The data run set an upper limit of $4 \times 10^{-43}$ on the WIMP-nucleon cross section for a WIMP mass of $60 \mathrm{GeV} / \mathrm{c}^{2}$. Upper limits to the rate of background neutrons have also been determined. 


\section{Chapter 1}

\section{Introduction}

\subsection{The Dark Side of the Universe}

There's more to the Universe than the eye can see. Recent astronomical evidence indicates that only $5 \%$ of the cosmos is known to us while $95 \%$ remains stubbornly hidden from view. Of this $22 \%$ constitutes the missing mass of the Universe, the so-called dark matter.

Theoretical predictions suggest that this unknown mass could be made of a generic class of particles called Weakly Interacting Massive Particles (WIMPs), relics of the Big Bang that still exist today. In the particle accelerator that was the early Universe, these particles were continuously produced and destroyed through self-annihilation, maintaining an equilibrium density.

As the Universe expanded and cooled, their abundance began to drop. When their number-density was too low for self-annihilations to occur, these particles froze out, allowing them to be available for detection even today.

Supersymmetry suggests a possible WIMP candidate in the lightest supersymmetric partner, the neutralino. Weakly interacting, massive and, in most cases, stable, this particle holds promise to be make up the missing mass in the Universe. 
WIMPs are believed to exist in a spherically-symmetric distribution in the galaxy, making up a dark matter cloud through which the solar system, and hence the Earth, moves. Occasionally a WIMP would experience a collision with the atomic nuclei of an Earth-based detector passing through the WIMP wind, leading to a detectable signal.

The Cryogenic Dark Matter Search looks for WIMPs using position-sensitive germanium and silicon crystals which simultaneously measure the charge and phonon signals that occur when a particle interaction takes place. These very rare WIMP interactions with matter are sought to be detected in an environment swamped with backgrounds. It does not help that the energies deposited in such interactions are of the order of a few $\mathrm{keV}$. These challenges are tackled using a two-pronged strategy - cooling the detectors to milli-Kelvin temperatures to improve sensitivity to low-energy events and operating the experiment in a shielded environment deep underground in a bid to reduce particle backgrounds.

Crucial to a rare particle search such as this is estimating the backgrounds seen by the experiment and the methods adopted to discriminate against them. Of special concern are backgrounds arising due to neutrons because these particles tend to mimic the WIMP signal in the detectors. With both particles giving rise to nuclear-recoil events, the event-by-event discrimination capability of the detector, so effective in the case of $\gamma$ and $\beta$-induced backgrounds, fails to distinguish between a neutron and a WIMP. We use Monte Carlo simulation techniques in conjunction with actual data to estimate, and subtract, any backgrounds arising due to neutrons.

The use of two different detecting material aids this process of sifting out the neutrons from likely dark matter candidates. Single-scatter events in the germanium detectors could arise either from neutrons or WIMPs while single-scatters in the silicon detectors are more likely to be a consequence of the former. Multiplescatters occurring in more than one detector need naturally be neutrons; it is highly unlikely that the very low interaction rate of a WIMP would allow it to undergo two scatters in rapid succession. 
By providing ratios of likely single-scatters in germanium to those in silicon, and likely single to multiple-scatters, the simulations provide a means of subtracting the neutron contribution.

The CDMS WIMP-search consists essentially of reducing the few million events seen during the course of an experimental run to a sample of bona-fide singlescatter nuclear-recoil events in germanium coupled with an independent estimate of the number of such recoils arising from neutrons.

This dissertation considers the use of computer-based simulations to help make such an estimate of the neutron backgrounds. The path it traces parallels the course of two stages in the CDMS-II search for WIMPs.

The first part of the experiment was conducted at the 17 meters-of-waterequivalent deep Stanford Underground Facility (SUF) at Stanford University. While this was enough to block the hadronic component of the cosmic-ray muon flux, the relatively shallow depth ensured that the limiting background seen by the experiment arose from high-energy neutrons produced from muon interactions with the rock of the cavern[1].

This work details how simulations of various neutron populations seen by the detectors helped inform the data analysis and confirm that the WIMP-candidate events seen in a recent experimental run at the site were consistent with high-energy neutrons punching through the shielding material and leaving traces akin to WIMP signals in the detectors.

The results from analysis of data emerging from the experimental run at Stanford excluded new parameter space for WIMPs of masses between 8-20 GeV/c ${ }^{2}$ [2] and set limits on the WIMP-nucleon cross section that were lower than previous CDMS results for higher WIMP masses.

Moving deeper underground to the 2090 m.w.e Soudan Underground Laboratory led to a considerable reduction in muon, and hence neutron, flux and a consequent improvement in the sensitivity to WIMP detection. Analysis of various populations in the data confirmed that the just-concluded first experimental 
Run at the deep site considerably reduced the neutron backgrounds seen by the experiment.

The rest of the dissertation details the use of simulations in predicting expected neutron backgrounds and informing the analysis of data from the first physics run at a deep site. The first run extended the sensitivity to dark matter detection and set upper limits of $4 \times 10^{-43}$ on the WIMP-nucleon cross section for a WIMP mass of $60 \mathrm{GeV} / \mathrm{c}^{2}[3]$, the best seen by any dark matter experiment in the world to date.

\subsection{Searching for WIMPs}

Searching for a particle that is believed to constitute a large proportion of the Universe but which, perversely, evades detection, is not the stuff of most Ph.D. dissertations. The way it became part of mine can be traced back to the first year of my graduate studies when I chose to study Superconducting Transition Edge Sensors as part of my course work for a class. Employing something as elementary as the linear variation of resistance with temperature in looking for exotic matter intrigued me enough to want to be part of the Cryogenic Dark Matter Search for my research experience.

When I first joined the group, my thesis advisor, Dan Akerib, asked me which particular aspect of the experiment interested me the most. I had quickly come to realize that the project had a bit of everything - some parts cosmology, some parts condensed-matter physics, some cryogenic technology, some more particle physics and a large dash of computer-based simulations and analytical work. I expressed interest in learning how to simulate the backgrounds that continuously bombard our experimental apparatus.

With Case having one of the two detector testing facilities of the collaboration, it made sense to first get a good grounding in detector characterization. Cooling the Kelvinox 400 dilution refrigerator located in the basement of Rocke- 
feller to a base temperature of around $20 \mathrm{mK}$ is the first step in this process. Each CDMS detector has four phonon sensors whose transition temperatures, normal and superconducting resistances and critical currents had to be measured at base temperatures. Energy calibrations were done by shining collimated gamma sources such as ${ }^{241} \mathrm{Am}$ with distinctive lines at 14, 18 and $60 \mathrm{keV}$. Along with other sources such as ${ }^{57} \mathrm{Co}$ and ${ }^{137} \mathrm{Cs}$, the resolution of the charge and phonon channels, information about the position and energy distribution in the sensors and the distribution of yield were studied.

Side by side, I began learning how to use the GEANT3 particle-transport code to be able to simulate the production and propagation of different particles through our experimental apparatus.

Though my first exercise in modelling an existing experiment involved the propagation of photons incident from a ${ }^{57}$ Co source onto three ZIP detectors in a standard test-facility calibration run, I quickly moved to the main task at hand working on simulating the neutron background for the ongoing WIMP-search run at the Stanford Underground Facility. The shallow depth of the facility ensured that neutrons were our limiting background at this site. As our detectors were unable to distinguish between events arising from neutrons or the hypothetical WIMPs, simulations were our only means of understanding these backgrounds.

It took a while for me to appreciate what a critical part these studies played in the final dark matter analysis. It helped that a finishing graduate student in our group, Thushara Perera, had till then been working on such simulations. It was from Thushara that I learned how to code in the geometry of the experimental setup, throw different classes of neutrons at the detectors and see how they responded to these backgrounds.

I began with coding in the geometry of the experimental run in progress at the Stanford facility. Next I simulated the calibration runs using a ${ }^{252} \mathrm{Cf}$ source. These runs are necessary to characterize the detector response to nuclear-recoil events and also serve as a cross-check of the simulations by comparing them with 
data. I coded in the various calibration runs that occurred during the course of the data taking and compared rates and spectra with the analyzed data to ensure the computer modelling was progressing along the right lines.

Subsequently, I started simulating the population of neutrons which arise due to cosmic-ray muon interactions with the shield. Vetoed out of the dark matter analysis, these neutrons occur throughout the duration of a run and serve as a second calibration measure.

Lastly, I began work on simulating the "punch-throughs" - neutrons of energies greater than $50 \mathrm{MeV}$ that are produced from muon interactions with the rock which punch through the shielding and serve as the limiting background seen by the experiment.

The results of the simulations were converted to a Matlab-friendly output to use them in tandem with the data. I was helped greatly in this by Richard Schnee who is the analysis mainstay of the collaboration.

By this time, the shifting of the experiment to the deep site at the Soudan Underground Laboratory in Minnesota was on everyone's radar screen and soon I began travelling there to help in the commissioning of the next stage of CDMS II.

At Soudan, I was involved in different things, actually each trip seems to involve some aspect of the experiment that was far removed from the earlier trip. From analyzing data as it was churned out in the early stages of the run to doing an all nighter in the mine during a Helium fill, I covered the entire gamut of the experience that is CDMS@Soudan.

For the current experimental run at Soudan, my contribution went along two parallel tracks. As before, I simulated the neutron background for the experiment. This involved coding in the geometry of the Soudan icebox and running simulations of the various neutron populations likely to be seen in the mine. In tandem, I worked on data analysis, helping to define data cuts, checking the efficiencies against the neutron-calibration simulations and studying likely neutron candidates in the data. 
The combination of deep site and relatively short duration of the first data run ensured that neutrons were yet to be a problem at this site. But with increased exposure, we would expect to start seeing first the muon-coincident "internal" neutrons and subsequently the more worrisome "punch-throughs". Dan suggested that my thesis include examining the impact of these neutrons on the subsequent CDMS experimental runs at Soudan. Using the current simulations as my basis, I sought to set an upper limit on the rate of background neutrons likely to be seen at Soudan.

\subsection{Layout of the thesis}

As specified earlier, this thesis focuses on the neutron backgrounds seen at the shallow and deep sites of the CDMS WIMP-search.

Every experimental probe has a theoretical underpinning to it. Chapter II examines the theoretical basis of the dark matter search - what makes us believe most matter is dark, why it could be made up of WIMPs and how we try to detect it.

The next chapter presents specifics of the first stage of the CDMS-search at a shallow site, including the detectors used and the challenges faced in reducing backgrounds incident on them.

Chapter IV focuses on the neutron backgrounds and the variation of neutron flux with depth.

As rejecting neutrons is something the detectors are ill-equipped to do, we use computer-modelling instead. Chapter $\mathrm{V}$ discusses the simulation techniques used to quantify the neutron contribution and why we can believe these models.

This provides a segue to Chapter VI which elaborates on how these simulations inform the data, and the dark matter analysis, for the WIMP-search at a shallow site

Chapter VII goes further underground to the deep site at Soudan where 
the principal difference is the dramatic reduction in the neutron backgrounds. The geometry and shielding of the experiment are examined again, but in the context of this knowledge.

Chapter VIII provides an in-depth look at the analysis methods applied to candidate neutron populations in the data and how this information, coupled with simulations, helped make estimates of the expected neutron flux at this site as well as improve sensitivity to WIMP detection.

The final chapter looks ahead to greater exposure, better constraints on the backgrounds and the hope of a possible WIMP detection. 


\section{References}

[1] D. Abrams et al., Phys. Rev. D, 66, 122003 (2002)

[2] D. S. Akerib et al., Phys. Rev. D, 68, 082002 (2003).

[3] D. S. Akerib et al., astro-ph/0405033 (2004). 


\section{Chapter 2}

\section{The Dark Matter Problem}

\subsection{The State of the Universe}

A plethora of astrophysical observations in recent times have helped paint a picture of the Universe that is as intriguing as it is disconcerting. It is now clear that the Universe is essentially non-visible, with over $95 \%$ of it made of something fundamentally different from the ordinary stuff of which we are made. Of this, $73 \%$ constitutes a mysterious dark energy that is accelerating the expansion of the cosmos[1]. The rest is dark matter, the missing-mass believed to be made up of non-conventional, exotic particles postulated by theory but still resisting the reach of experimental detection. Ordinary matter makes up just $5 \%$ of the total matterenergy density of the cosmos, with the luminous component constituting barely $1 \%$ of the whole [2].

This chapter looks at the dark matter problem in some detail. It considers the present-day understanding of the Universe and the cosmological parameters based on studies of galaxy clusters, supernovae and the microwave background radiation. It presents experimental evidence that most of the Universe may be made up of a non-luminous component that we call dark matter. It then explains why dark matter is considered to be both cold (i.e., non-relativistic) and exotic (i.e., non- 
baryonic), based on our understanding of Big Bang Nucleosynthesis and the largescale structure of the Universe. The chapter examines the theoretical motivations that govern the choice of Weakly Interacting Massive Particles (WIMPs) as possible dark matter candidates. It indicates that supersymmetry offers a possible WIMP candidate in the lightest supersymmetric particle. Also covered are the reasons why WIMPs may be detected and the means adopted to detect them.

\section{$2.2 \quad$ A Flat Universe}

The current-day understanding of the Universe is that, on large scales, it is homogenous, i.e., a point in space is indistinguishable from any other point, and isotropic, i.e., there exists no preferred direction [3][109]. We also know that it is expanding, with the expansion increasing in time. An expanding Universe with an uniform energy-density and curvature, and with no preferred orientation, may be described by the Robertson Walker metric:

$$
d s^{2}=-d t^{2}+a(t)^{2}\left(\left(d \chi^{2}+\Sigma^{2}(\chi) d \Omega^{2}\right)\right.
$$

where $\chi$ is a dimensionless radial co-ordinate, $\mathrm{d} \Omega$ is an infinitesimal solid-angle element and $\mathrm{a}(\mathrm{t})$ is a scaling factor that describes the expansion of the Universe. The value of $\Sigma$ may be either $\sin (\chi)$ which describes a closed, spheroidal Universe, or $\sinh (\chi)$ describing an open, hyperboloid universe with $a$ as its radius of curvature. In the limit of $\chi \rightarrow 0, \Sigma(\chi) \rightarrow \chi$, giving a flat, Euclidean universe. From General Relativity we have,

$$
H^{2} \equiv\left(\frac{1}{a} \frac{d a}{d t}\right)^{2}=\frac{8 \pi G}{3} \rho+\frac{\Lambda}{3}-\frac{k}{a^{2}}
$$

where $G$ is the gravitational constant and $\Lambda$ is the cosmological constant identified with a non-zero vacuum energy-density having pressure $p_{\Lambda}=-\rho_{\Lambda}$. $\mathrm{H}$ is the Hubble 
parameter describing the expansion rate of the Universe. Here $\rho$ and $p$ are the density and pressure of the constituents of the Universe respectively. Equation 2.2 is called the Friedmann equation. It informs us that the kinetic energy of expansion of the Universe is equal to its gravitational potential energy, which is given by the matter-density, the curvature-density and the vacuum-energy density. The time derivative of the Friedmann equation and the conservation of energy can be used to express the acceleration rate of the Universe:

$$
\frac{1}{a}\left(\frac{d^{2} a}{d t^{2}}\right)=-\frac{4 \pi G}{3}(\rho+3 p)+\frac{\Lambda}{3} .
$$

Equation 2.3 is a term expressing the acceleration of the expansion of the Universe. It shows how normal matter slows down the expansion of the Universe while the cosmological constant accelerates it.

We define $\mathrm{k}$ as the curvature index such that $\mathrm{k}=1$ gives a closed Universe, $\mathrm{k}=-1$ gives an open Universe while $\mathrm{k}=0$ gives a flat Universe. The normalized densities of matter, curvature and cosmological constant are given by:

$$
\begin{gathered}
\Omega_{m} \equiv \frac{8 \pi G \rho_{o}}{3 H_{o}^{2}} \\
\Omega_{k} \equiv \frac{-k}{a_{0}^{2} H_{0}^{2}} \\
\Omega_{\Lambda} \equiv \frac{\Lambda}{3 H_{0}^{2}}
\end{gathered}
$$

where $\mathrm{H}_{0}$ and $\rho_{o}$ are the present-day values of the Hubble constant and the matter density of the Universe respectively. Thus, Equation 2.2 becomes: 


$$
\Omega_{m}+\Omega_{k}+\Omega_{\Lambda} \equiv 1
$$

This implies that the total-density parameter of the Universe today $\Omega=\Omega_{m}+\Omega_{\Lambda}$ is directly related to its present curvature. A flat universe has $\Omega=1$ and the kinetic energy of expansion is exactly balanced by the gravitational potential. An open Universe has $\Omega<1$ and a closed Universe has $\Omega>1$. The value of the density that separates the three cases, $\rho_{c}$, is the critical-density of the Universe.

Our current understanding is that we live in a flat Universe where the total amount of matter and energy-density $\Omega \simeq 1$. $\Omega$ comprises a matter component $\Omega_{m}$ $\simeq 0.3$ and a vacuum-energy component $\Omega_{\Lambda} \equiv 0.7[1][5]$. The next section summarizes some of the recent experimental observations that helped us arrive at this conclusion.

\subsubsection{Cosmic Microwave Background Radiation}

The Universe was created thirteen billion years ago in a cataclysmic explosion that is popularly known as the Big Bang. The explosion, and the expansion that followed it, created photons as well as matter in the form of leptons, baryons and various non-baryonic particles. That the Big Bang occurred is evinced from the measurement of an isotropic radiation that permeates the entire Universe known as the Cosmic Microwave Background (CMB) Radiation. By studying this radiation, we are able to examine the conditions in the Universe 400,000 years after the Big Bang, known as the 'surface of last scattering'. Though the virtual isotropy of the CMB is better than one part in 100,000, there exist tiny ripples in the temperature of the microwave sky which provide us information about the seed fluctuations that existed at the time of the de-coupling of matter and radiation, much prior to the formation of galaxy structure. These seed fluctuations grew by gravitational attraction into the stars, galaxies and galaxy clusters we see today. Measurements of the 
anisotropies in the CMB may thus be employed to determine various cosmological parameters.

There have been various studies in recent years of the angular anisotropy power spectrum of the $\mathrm{CMB}$ characterizing the size of the temperature fluctuations. Peaks in the power spectrum are indicative of the harmonics in the sound waves that filled the early Universe. Until 400,000 years after the Big Bang, the Universe was so hot that matter and radiation were entangled in a primordial soup in which sound (pressure) waves could vibrate. At the time of the de-coupling of matter and radiation, these pressure waves left traces of their existence in the temperature fluctuations seen in the CMB radiation today. Cosmological models predict the existence of acoustic peaks in the angular power spectrum. The relative position and height of the peaks provide estimates of $\Omega$ and $\Omega_{m}$. Studies of the peaks in the CMB angular anisotropy power spectrum by experiments such as BOOMERANG[112], DASI [7] and MAXIMA[8] point to $\Omega_{m}+\Omega_{\Lambda} \simeq 1$,suggesting a flat Universe. This has been corroborated by the Wilkinson Microwave Anisotropy Probe launched in 2001 to study the microwave background sky with unprecedented accuracy. The WMAP results indicate that the matter-energy density $\Omega_{m}=0.27 \pm 0.04$ while the vacuumenergy density is $\Omega_{\Lambda}=0.73 \pm 0.04$ giving a total density of $\Omega=1.02 \pm 0.02[1]$.

\subsection{A Dark Universe}

The previous section indicated that the total matter-density of the Universe $\Omega_{m} \simeq 0.3$. The matter-density due to the luminous component is given by $\Omega_{\text {lum }} \simeq$ 0.005. Clearly, most of the matter in the Universe is made up of what is known as dark matter. This section examines the experimental evidence that helped arrive at this conclusion. 


\subsubsection{The First Evidence}

When certain astronomical observations made within the conventional framework of gravity gave results at complete variance with the prevalent understanding of the matter-content of the Universe, scientists were forced to confront the idea that we live in an overwhelmingly "dark" Universe. The first suggestion came in 1933 with Zwicky's studies of the radial velocities of galaxies in the Coma Cluster[113]. Zwicky measured the radial velocities for a set of galaxies in the region of the cluster, and computed the radial velocity dispersion. He then applied the virial theorem (explained subsequently in Section 2.3.4) to estimate the total mass of the cluster which he found to be substantially greater than the sum of the masses of the individual galaxies. This led him to suggest that most of the mass in the cluster was non-luminous or dark. This claim was supported in 1936 by Smith [10], who observed galaxies in the Virgo cluster moving faster than expected. Studies of the mass-to-light ratio by Oort [11] also indicated that $90 \%$ of the mass in the local group of spiral nebulae was missing.

Studies of the rotation curves of spiral galaxies in the 1970s proved further evidence. Rubin and Ford [12] showed that the gravitational force generated by the luminous mass was too less to account for the observed rotational speed of certain spiral galaxies. The plot of radius versus velocity flattened out instead of trailing down at large radii, implying that the enclosed mass increased with radii even where no luminous matter was seen. This suggested that large amounts of dark matter resided in the extended halos that surround visible stars, an hypothesis

confirmed by computer simulations of the stability of galactic disks by Ostriker and Peebles[13].

Subsequent astronomical observations further supported the case for nonluminous matter. Today, most scientists agree that most of the matter in the Universe is dark with strong evidence for this being provided by studies of rotational curves of spiral galaxies and galaxy clusters. 


\subsubsection{Rotational Curves of Spiral Galaxies}

In a spiral galaxy, the stars are concentrated in a thin luminous disk, moving in nearly circular orbits around the galaxy center. The optical radius of such a galaxy, $\mathrm{R}_{\text {opt }}$, is the radius containing $83 \%$ of the luminous matter content of the galaxy. This is about $10 \mathrm{kpc}$ for typical luminous spiral galaxies. Beyond this, clouds of neutral hydrogen are visible till about $20-30 \mathrm{kpc}$. The velocities of stars are measured from the red-shifts of the atomic structure lines in the visible and near infra-red region. For the clouds of interstellar hydrogen, the fine structure line $(\lambda \sim 21 \mathrm{~cm})$ is used.

The gravitational mass is obtained by observing the motion of stars and gas clouds as they orbit around the center of the galaxy. At a large distance from the center of the galaxy, the gravitational potential should be that produced by a central point mass. If $\theta$ is the rotational velocity, $\mathrm{M}=$ galactic mass, $\mathrm{G}=$ universal gravitational constant and $\mathrm{R}=$ galacto-centric radius then we should have:

$$
\frac{\theta^{2}}{R}=\frac{G M}{R^{2}}
$$

The $\theta \propto 1 / \sqrt{R}$ variation of the velocity of stars with the distance from the center of the galaxy represents the rotation curve of the galaxy[14]. As indicated in the equation, it was expected that the rotational velocity, and hence the masscontent of the galaxy, would fall off with increasing radial distance. Instead the curve flattens out at large distance from the center. Rotation curves for 1100 spiral galaxies were measured by Persic and Salucci[15]. They observed the flattening out of the rotation curve at distances greater than $\mathrm{R}_{\text {opt }}$ unlike the expected decline. At such distance, the luminous matter is unable to trace out the observed curve. Most spiral galaxies show flat rotation curves out as far as we can trace them, even where no more stars are visible. Figure 2.1 shows the rotation curve of the spiral galaxy M33 [44]. Shown in the figure is the observed rotation curve and the expected curve from luminous matter alone. The flattening out of the curve is indicative 
of a substantial presence of the non-luminous, 'dark', component of matter in the galaxy.

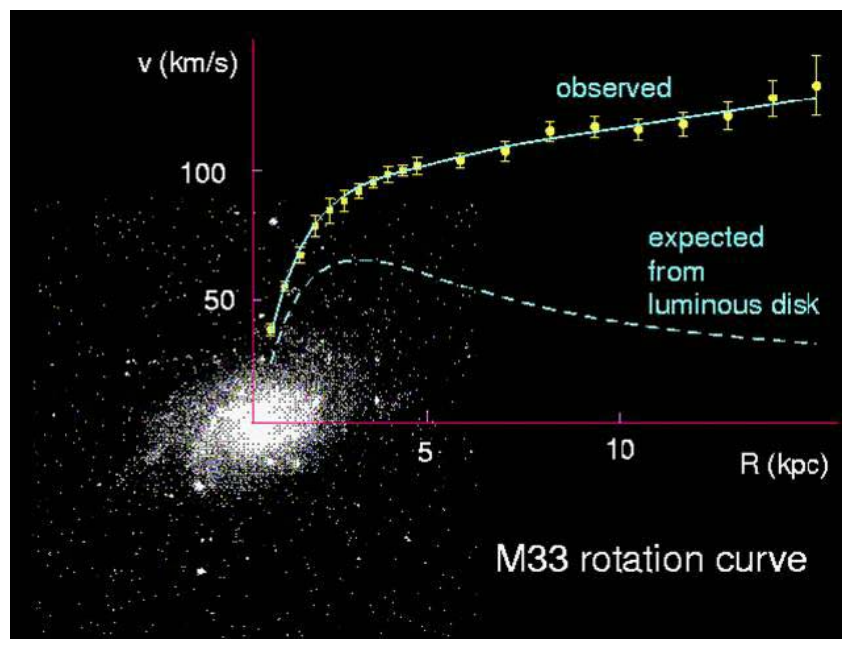

Figure 2.1: Rotation curve of the spiral galaxy M33 (taken from [44]).

\subsubsection{Elliptical galaxies}

Another source of evidence of the existence of dark matter are elliptical galaxies. Elliptical galaxies are some of the most massive galaxies in the Universe and are often found in galaxy clusters. They are often described as an amorphous distribution of stars that move in randomized orbits, unlike the coherent directionality shown by stars in spiral galaxies. The mass content of an elliptical galaxy may be determined from the stellar velocity dispersion and the halos of ionized, X-ray emitting gases surrounding these galaxies. As these hot gaseous halos are considered to be in hydrostatic equilibrium, measurements of the density and temperature distributions provide constraints on the distribution of mass in the galaxy. If $\rho_{g}(r)$ is the distribution of the density of the gas, $\mathrm{p}_{g}(r)$ is the gas pressure, $\mathrm{M}(\mathrm{r})$ is the total mass enclosed at distance $\mathrm{r}$ and $\mathrm{T}$ is the observed $\mathrm{X}$-ray temperature, then 


$$
\frac{d p_{g}}{d r}=-\frac{G M(r) \rho_{g}(r)}{r^{2}}
$$

Under thermal equilibrium, gas pressure and temperature are related by the ideal gas law:

$$
p_{g}=\frac{k_{B} T \rho_{g}}{\mu m_{p}}
$$

where $\mu m_{p}$ is the average ion mass and $\mathrm{T}=$ temperature of the halo. Hence:

$$
\frac{d}{d r}\left(\frac{k_{B} T \rho_{g}}{\mu m_{p}}\right)=-\frac{G M(r) \rho_{g}(r)}{r^{2}} .
$$

If the matter-content of the galaxy was determined by the luminous component alone, then the stars should have a density distribution consistent with thermal equilibrium. Both the stars and gas would move in the same gravitational well and have the same velocity dispersion:

$$
\sigma_{*}^{2}=\frac{k_{B} T}{\mu m_{p}}
$$

where $\sigma_{*}$ is the velocity dispersion of stars. Measurements of $\sigma_{*}$ and $\mathrm{T}$ for various elliptical galaxies[16] indicated that the above relation was not satisfied. Stars were found to have about half the velocity dispersion they should have had according to the equation. The assumption that dark matter halos in the galaxies provides the gravitational well necessary for the observed temperature allows for a more reasonable agreement with the halo [17].

\subsubsection{Clusters of galaxies}

Galactic clusters are aggregates of a few hundred to a few thousand galaxies, gravitationally bound to each other and otherwise isolated in space. The relative 
contribution of the dark matter component in a galactic cluster is specified by the Mass-to-Light Ratio (M/L), a term giving the total amount of mass relative to the total light within a given scale. By considering the $\mathrm{M} / \mathrm{L}$ ratio at different scales, we arrive at the conclusion that most of the Universe is made up of a non-luminous component. The $\mathrm{M} / \mathrm{L}$ ratio increases from the luminous parts of the galaxies to their fainter halos, and increases further when considering local groups of galaxies and galaxy clusters[18]. For galaxies, the M/L ratio tends to $>10 h \frac{M_{\odot}}{L_{\odot}}$ while for galactic clusters, it is in the range of $(250-450) h \frac{M_{\odot}}{L_{\odot}}$. Here $\mathrm{h}$ is the dimensionless quantity called the Hubble parameter given by:

$$
h=\frac{H_{0}}{100 k m s^{-1} M p c^{-1}}
$$

The methods used to determine the mass-to-light ratios from galaxy clusters include measuring the velocities of individual galaxies in the clusters at dynamical equilibrium[19] and estimating the total cluster mass from the virial theorem. According to the theorem, the kinetic and potential energies of a system are related by

$$
<T>=-\frac{1}{2}<V>
$$

where $\langle\mathrm{T}\rangle$ is the average kinetic energy derived from the dispersion in the velocities and $\langle\mathrm{V}\rangle$ is the average potential energy. The latter is used to determine the mass of a cluster.

The X-ray emission of hot intra-cluster gas, assumed to be at hydrostatic equilibrium, can also be used to estimate the mass of clusters [20]. X-ray profiles of the gas are measured and then fit to temperature and density distribution models to determine the mass of the cluster.

The mass of a cluster can also be determined using gravitational lensing methods[21]. When light rays pass through large gravitational masses such as 


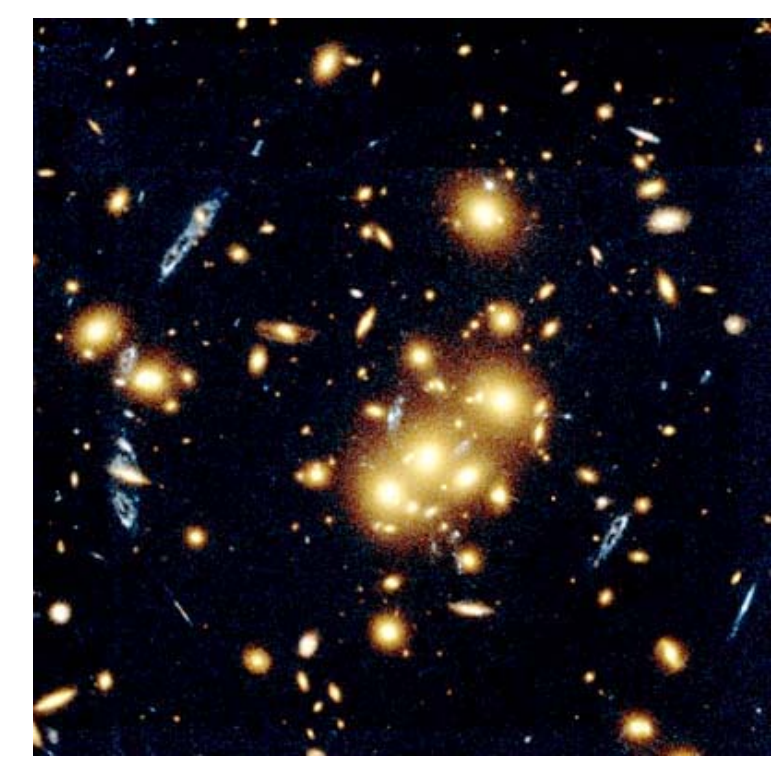

Figure 2.2: Image of the galaxy cluster $0024+1654$ taken by the Hubble Space Telescope demonstrates gravitational lensing by large galaxy clusters. Light from distant galaxies passes by the gravitational mass of the cluster and gets bent, creating a lensing effect (taken from [45]).

galaxy clusters, they are deflected by the enormous gravitational field produced by the cluster, in a manner similar to the way an optical lens bends light to form an image. By analyzing the amount of bending of light, we can then determine the mass of the galaxy cluster. The estimates indicate that there is far more mass exerting a gravitational effect than suggested by the luminous component. Figure 2.2 illustrates the gravitational lens effect produced by Cluster 0024+1654 as seen through the Hubble Space Telescope. Light from distant galaxies is bent as it passes by the cluster giving rise to a lensing effect. 


\subsection{Cold Exotic Dark Matter}

\subsubsection{Big Bang Nucleosynthesis}

The experimental evidence presented earlier on in the chapter indicates that a substantial part of the Universe is made up of a non-luminous component. By considering the constraints set on the amount of luminous matter in the Universe based on astronomical observations of galaxies and galaxy clusters alone, we conclude that $\Omega_{\text {lum }} \sim 0.005$. Recent measurements of clusters have indicated that the matter density of the Universe is $\Omega_{\mathrm{m}}=0.325 \pm 0.034[46]$. Clearly the deficit is made up by what we call dark matter.

To determine the nature of most of this dark matter, we turn to Big Bang Nucleosynthesis. This term refers to the calculations of the abundances of the light elements such as ${ }^{2} \mathrm{H},{ }^{3} \mathrm{H},{ }^{4} \mathrm{He}$ and ${ }^{7} \mathrm{Li}$ relative to photons within the framework of the Big Bang model of the Universe. Less than one second after the Big Bang, the neutron-to- proton ratio is maintained in thermal equilibrium through the following reactions:

$$
\begin{aligned}
& p+e^{-} \leftrightarrow n+v \\
& n+e^{+} \leftrightarrow p+v
\end{aligned}
$$

About 1 second after the Big Bang, these reactions become slower than the expansion rate of the Universe, and the neutron-to-proton ratio freezes out at about 1:7. The temperature of the Universe falls from the phenomenally hot $10^{32}$ Kelvin to $10^{9}$ Kelvin, below the nuclear binding energies, suppressing the numbers of photons with energies high enough to disassociate these nuclei. Light elements begin to form. Figure 2.3 gives the evolution of the abundances of light elements over time (and temperature) in the first few minutes after the Big Bang.

By the time the temperature of the Universe fell to $\mathrm{T} \approx 0.03 \mathrm{MeV}$, the light elements ${ }^{4} \mathrm{He},{ }^{3} \mathrm{He},{ }^{3} \mathrm{H},{ }^{7} \mathrm{Li}$, and ${ }^{7} \mathrm{Be}$ have established their final abundances. 


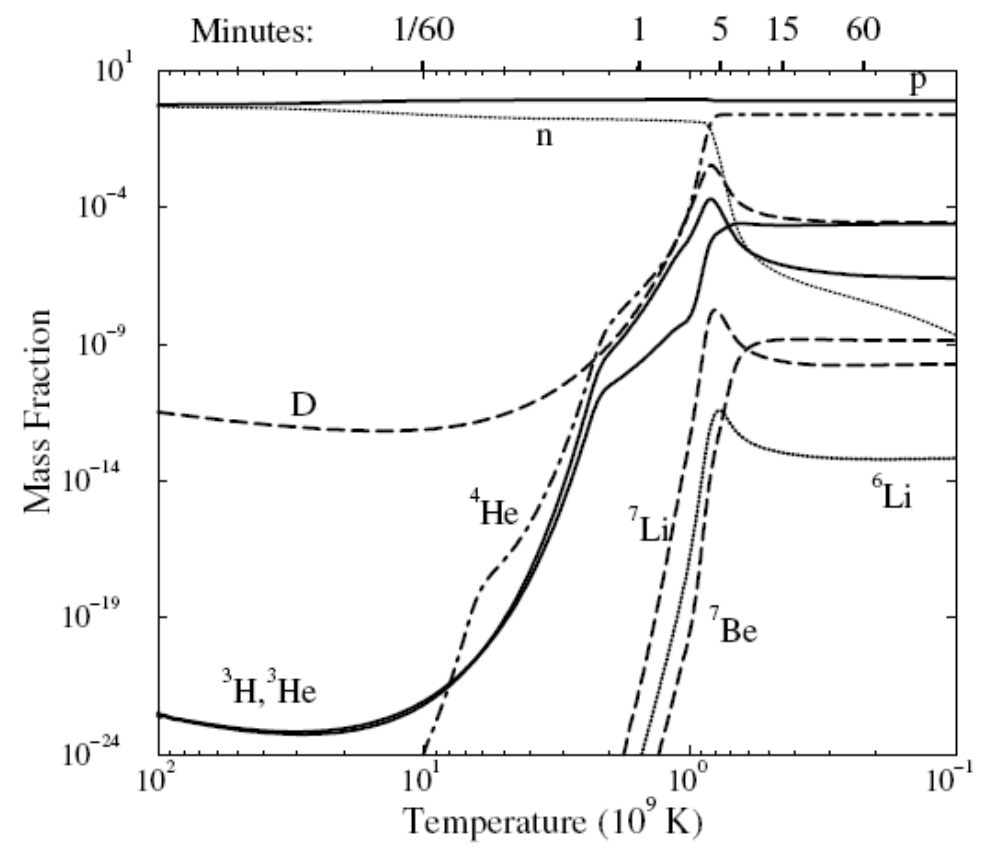

Figure 2.3: Evolution of the abundances of light elements over temperature and time (taken from [22]) 
When all the neutrons have been used, the intermediate nuclei do not form but the reactions through which they combine continue. The amount of leftover deuterium is very sensitive to the density because this gets frozen in once the processes through which deuterium and other elements that form helium stop. Hence, the neutronto-proton ratio set at the time of the freeze out is important in deciding the final abundances of these elements in the early Universe. For a neutron to proton ratio of $1: 7$ at the time of the formation of these elements, $25 \%$ of the mass of the Universe ends up in helium. Measurements of the abundance of the light elements in the Universe can, therefore, place limits on the baryonic density. Increased accuracy in astrophysical measurements have placed tighter constraints on the baryonic matterdensity. These include studies of quasars[22] and the abundance of ${ }^{2} \mathrm{H}$ in high redshift clouds[23]. Current measurements place the constraints on the baryonic matter- density as:

$$
0.018<\Omega_{b} h^{2}<0.023
$$

The WMAP findings of $\Omega_{b} h^{2}=0.0224 \pm 0.0009$ or $\Omega_{b}=0.044 \pm 0.004$ corroborate the earlier results. Given our knowledge of the matter-density $\Omega_{m} \simeq 0.3$, this suggests that most of the matter in the Universe is non-baryonic in nature. Given that $\Omega_{\text {lum }} \simeq 0.005$, this also suggest that most of the baryonic matter as well happens to be dark.

\subsubsection{Cold and hot dark matter}

That dark matter is principally made up of an exotic form of matter was shown in the earlier section. Dark matter candidates that satisfy this condition include neutrinos, axions and Weakly Interacting Massive Particles (WIMPs). Unlike the latter two cases, neutrinos have been detected experimentally and typically have masses of around $10^{-5} \mathrm{eV}$. However, we rule out neutrinos as making up the bulk of dark matter in the following way: Dark matter candidates may be classified as 
'hot' or 'cold' based on their energy at the time they de-coupled from the rest of the Universe. If they had been moving at relativistic speeds at that time, they are known as hot. Neutrinos fall in this category.

The present Universe points to dark matter being predominantly cold. This is because tiny fluctuations in the matter-density of the very early Universe have evolved into the large scale structure we see today. Fluctuations in the baryonic matter density show up as anisotropies in the cosmic microwave background radiation. However, these variations are not enough to create the clumped nature in the distribution of matter that we see today. We need to see such fluctuations in the density of matter that does not couple to photons. This would allow for the evolution of the present-day structure of the Universe without affecting the amplitude of the anisotropies seen in the temperature of the CMB radiation. This suggests that dark matter is mainly cold.

Axions and WIMPs are cold dark matter candidates. Axions are particles proposed to solve the problem of the observed absence of $\mathrm{CP}$ violation in the strong interaction. Experimental observations have set the constraints for the axion mass to be $10^{-6}-10^{-3} \mathrm{eV}[25]$. They are considered a good candidate to explain the dark matter in the Universe.

\subsection{WIMP Dark Matter}

\subsubsection{WIMP relic density}

A promising dark matter candidates is the Weakly Interacting Massive Particle. WIMPs are generic particles that are believed to have been created in the earlier stages of the Big Bang. They are weakly interacting with matter and massive, about 200 times the mass of a proton. Hence the term WIMP. The reasons for their relic abundance today is given by the freeze-out argument [26]. The following explanation has been drawn, in part, from [27] and [28]. 
In the early Universe, WIMPs were both at chemical and thermal equilibrium. The rate of their creation was balanced by their annihilation rate allowing them to be in chemical equilibrium. Collisions with other neighboring particles in the dense plasma that was the early Universe allowed them to maintain thermal equilibrium. As the Universe expanded and cooled to a temperature much lower than that required to produce the particle, the annihilation rate began to exceed the rate of creation and the number-density of these particles began to decrease. Eventually, the density became small enough so as not to allow further annihilation, i.e., the expansion of the Universe caused the particle to fall out of thermal equilibrium giving it a non-zero relic density. The evolution of the number density of the particle over time is given by the Boltzmann equation:

$$
\frac{d n_{\chi}}{d t}+3 H n_{\chi}=-\left\langle\sigma_{A} v\right\rangle\left(n_{\chi}^{2}-\left(n_{\chi}^{e q}\right)^{2}\right) .
$$

where $\chi$ denotes the particle, $\mathrm{H}=$ Hubble constant, $n_{\chi}$ is the number-density and $n_{\chi}^{e q}$ is the number-density in thermal equilibrium[26]. Let us consider temperature as the variable instead of time

$$
t \rightarrow x \equiv \frac{m}{T}
$$

and the co-moving number-density instead of number-density

$$
n_{\chi} \rightarrow Y \equiv \frac{n_{\chi}}{s}
$$

where $\mathrm{s}$ is the entropy density. The parameter s scales inversely with the volume of the Universe when the entropy is conserved. Hence Equation 2.18 becomes:

$$
\frac{x}{Y_{e q}} \frac{d Y}{d x}=\frac{-n_{\chi}^{e q}\left\langle\sigma_{A} v\right\rangle}{H}\left(\frac{Y^{2}}{Y_{e q}^{2}}-1\right)
$$


Before freeze out, when the annihilation rate is large compared to the expansion rate, $\mathrm{Y}$ follows its equilibrium value. After freeze-out, $\mathrm{Y} \rightarrow$ constant which depends on the annihilation cross section. The larger the cross section, the longer $\mathrm{Y}$ follows its exponentially falling value at equilibrium and the lower the value of the relic density. The relic density is inversely proportional to $\left\langle\sigma_{A} v\right\rangle$ where $\sigma_{A}$ is the annihilation cross section and $v$ the relative velocity. This is illustrated in Figure 2.4 which shows the variation of the WIMP relic density with the annihilation cross section. The annihilation cross section thus informs us of the number density of the particle at freeze out. For WIMPs to have a relic density on the order of the critical density, the annihilation cross section is set by the weak scale

$$
m^{2}=\frac{1}{\left\langle\sigma_{A} v\right\rangle}
$$

In Equation 2.18, the term $3 H n_{\chi}$ gives the expansion of the Universe while $\left\langle\sigma_{A} v\right\rangle n_{\chi}^{2}$ describes the annihilation of the particle. At the time of freeze out:

$$
H n_{\chi}=\left\langle\sigma_{A} v\right\rangle\left(n_{\chi}^{2}\right)
$$

which implies that the expansion and the annihilation rates are equal. Equation 2.23 is called the freeze-out criteria. As the temperature at freeze-out is less than the rest-mass energy of the particle, this implies that WIMPs fell out of equilibrium after becoming non-relativistic. They may thus be classified as cold relics. For such cold relics, the relic abundance can be determined from the rate of annihilation at the time of the freeze-out. For a non-relativistic particle,

$$
n_{\chi}^{e q} \sim(m T)^{\frac{3}{2}}\left\langle e^{-m / T}\right)
$$

Also $\mathrm{H} \sim \frac{T^{2}}{M_{*}}$. Here 


$$
M_{*} \cong 2.4 \times 10^{18} \mathrm{GeV}
$$

is the reduced Planck's mass. The freeze-out thus occurs when:

$$
\frac{m}{T} \sim \ln \left[\left\langle\sigma_{A} v\right\rangle m M_{*}\left(\frac{m}{T}\right)^{1 / 2}\right] \sim 30
$$

using

$$
\frac{1}{2} m v^{2}=\frac{3}{2} T
$$

where $\mathrm{c}=\mathrm{k}_{B}=1$. This implies that WIMPs freeze out with velocity 0.3 .

The relic density of such a particle can thus be determined from its mass, which determines the time at which the particle fell out of equilibrium, and the annihilation cross section, which determines the temperature at freeze out. Using the conserved quantity $\frac{n_{\chi}}{s}$ and the freeze-out criteria, we get

$$
\left(\frac{n_{\chi}}{s}\right)_{f} \propto \frac{H_{f}}{\left\langle\sigma_{A} v\right\rangle T_{f}^{3}} \propto \frac{1}{m_{\chi}\left\langle\sigma_{A} v\right\rangle}
$$

where $\mathrm{T}_{f}$ is the freeze-out temperature and $\mathrm{s}$ is the entropy density such that $s \propto T^{3}$. The mass density of the particle today is given by

$$
\Omega \chi h^{2}=\frac{m_{\chi} n_{\chi}}{\rho_{c}}=\frac{\left(3 \times 10^{-27} \mathrm{~cm}^{3} s^{-1}\right)}{\left\langle\sigma_{A} v\right\rangle}
$$

Recent measurements from WMAP [5] have indicated that the cold dark matter density in the Universe is given by:

$$
\Omega_{C D M} h^{2}=0.113_{-0.009}^{+0.008}
$$




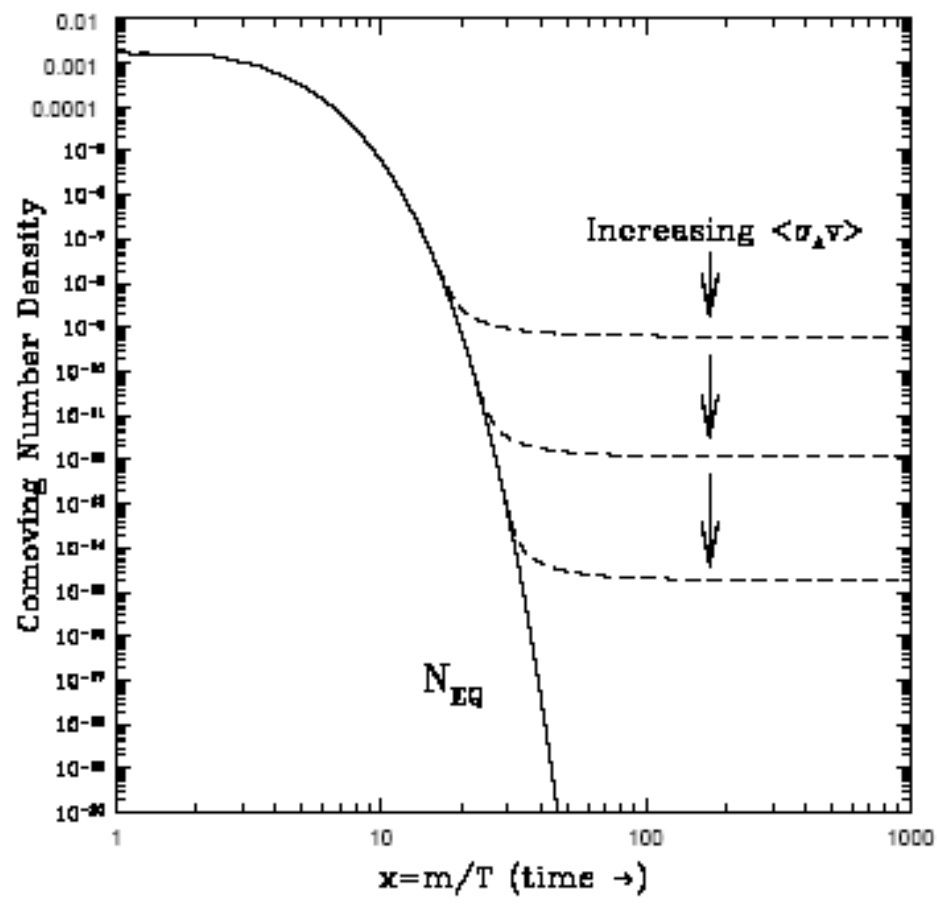

Figure 2.4: The number density of WIMPs in the early Universe vs. time. The solid line refers to the equilibrium density while the various dashed lines give the relic densities for increasing values of the annihilation cross sections (taken from [29]. 
If the cold dark matter in the Universe were made up a stable massive particle, then by Equation 2.28, its annihilation cross section $\left\langle\sigma_{A} v\right\rangle \sim 10^{-28} \mathrm{~cm}^{2} \mathrm{~s}^{-1}$. A typical weak interaction cross section is $\left\langle\sigma_{A} v\right\rangle \sim 10^{-25} \mathrm{~cm}^{2} \mathrm{~s}^{-1}$. This suggests that a particle showing interaction strengths characteristic of the weak force may be a viable dark matter candidate.

\subsubsection{The Neutralino as a Dark Matter Candidate}

It is now clear that WIMPs, if they exist, could make up a form of cold exotic dark matter. To describe their constitution, and properties, we need to move beyond the Standard Model of particle physics to find explanations in Supersymmetry. Supersymmetry is a theory that was postulated as a means of unifying the fundamental forces of nature such as the strong, electro-weak and gravitational forces. According to the theory, every particle has associated with it a massive superpartner whose spin differs by $1 / 2$. The particles are related by a supersymmetric transformation. Each fermion has a boson associated with it as a super-partner and a boson in turn has a fermion partner particle. So a fermionic quark will have a bosonic super-partner called the squark while the photon, which is a boson according to the Standard Model, has a fermionic partner called the photino.

In supersymmetry, baryon and lepton numbers of particles are not assumed to be conserved, instead R-parity is assumed to be conserved and

$$
R=(-1)^{3(B-L)+2 S}
$$

where $B$ and $L$ are baryon and lepton number operators, and $S$ is the spin operator for the particle. R-parity conservation implies that $\mathrm{R}=1$ for Standard Model particles while $\mathrm{R}=-1$ for their supersymmetric partners[29].

Insofar as the values of the parameters of the model are not known, there exist many theories of supersymmetry. For the purpose of understanding the nature of a possible dark matter candidate, it is enough to consider the Minimal Supersymmetric Extension to the Standard Model (MSSM). In the MSSM interpretation, 
every particle defined by the Standard Model plus a Higgs particle is assigned a supersymmetric partner. R-parity needs to be conserved as specified earlier. The lightest supersymmetric particle (LSP) is defined as a superposition of the neutral gaugino and the neutral higgsino and is called the neutralino[29]. It is stable, massive (30 -200 times the mass of a proton) and electrically neutral, and is theorized to have an annihilation cross section which yields a relic abundance that fits in with our understanding of the current cosmological parameters. A Majorana particle, the neutralino is its own anti-particle. Also, since R-parity is conserved, this implies that the Lightest Supersymmetric particle is stable, allowing for its present-day existence since its creation in the Big Bang. In short, it makes a natural candidate for dark matter[30][31].

\subsection{WIMP detection}

Supersymmetric models predict a wide range of neutralino mass and interaction cross sections with ordinary matter. While a WIMP has not been experimentally detected so far, there have been a slew of experiments world wide searching for the elusive particle. It is believed that WIMPs are distributed in an isothermal halo about a galaxy[32]. They are sought to be found either by direct detection methods that look for signals associated with the scattering of WIMPs by ordinary matter or by indirect searches that look for the products formed as a consequence of their annihilation such as neutrinos and gamma rays.

\subsubsection{Direct detection}

It is believed that dark matter clusters gravitationally in galactic halos, having a local energy-density given by $0.3 \mathrm{GeV} / \mathrm{cm}^{3}$ and moving with a RMS velocity of $220 \mathrm{~km} / \mathrm{s}$ with respect to the Earth's motion[33]. Using the two values as a normalization factor allows us to compute the flux of WIMPs as: 


$$
\Phi_{\chi}=6.6 \times 10^{4} \mathrm{~cm}^{-2} \mathrm{~s}^{-1} \frac{\rho_{\chi}}{0.3 G e V / \mathrm{cm}^{3}} \frac{100 \mathrm{GeV}}{m_{\chi}} \frac{v}{220 \mathrm{~km} / \mathrm{s}}
$$

The flux allows for a possible detection by an Earth-based detector looking for WIMPs. The maximum recoil energy from a WIMP scattering off a nucleus of a detecting material is given by:

$$
E_{\text {recoil }}=\frac{2 m_{\chi}^{2} m_{N}}{\left(m_{\chi}+m_{N}\right)^{2}} v^{2}
$$

where $\mathrm{m}_{\chi}$ is the mass of the dark matter particle and $\mathrm{m}_{N}$ is the mass of the recoiling nucleus. For WIMPs with a Boltzman velocity distribution of $220 \mathrm{~km} / \mathrm{s}$, the recoil energies recorded are typically of the order of a few $\mathrm{keV}$ to about a hundred keV[33]. As the recoil energies are low, WIMP detection usually proceeds through the process of elastic scattering with nuclei of atoms of materials like germanium and silicon. Here, the WIMP interacts with the target nucleus, causing it to recoil. The event rate per unit mass on a target of atomic mass A with cross section per nucleus $\sigma$ is

$$
d R=\frac{N_{0}}{A} \sigma v d n
$$

where $\mathrm{N}_{0}$ is the Avogadro's number. Therefore,

$$
R=\frac{N_{0}}{A} \sigma_{0} n_{0}\langle v\rangle
$$

for the case of zero momentum transfer. The WIMP interaction rate per unit mass is thus given by

$$
R=\sigma n\langle v\rangle
$$


where $\mathrm{n}$ is the number density of WIMPs, $\sigma$ is the WIMP-nucleus cross section and $\langle v\rangle$ is the average relative velocity between a WIMP and a nucleus. The number density can be represented as $\frac{\rho_{D}}{M_{D}}$ where $\rho_{D}$ is the local halo density taken as 0.3 $\mathrm{GeV} / \mathrm{cm}^{3}$ and $\mathrm{M}_{D}$ is the WIMP mass.

The WIMP-nucleus scatter occurs at the level of WIMP-quark interactions. The Feynman diagram for such interactions is shown in Figure 2.5. The scattering is coherent if the wavelength corresponding to the momentum transfer is comparable to the nuclear radius. For such a case, the WIMP-nucleus cross section is increased by $\mathrm{A}^{2}$ as compared to the WIMP-nucleon cross section. This applies only to the case of coherent spin-independent interactions which tends to dominate the cross section in most interaction models.

The WIMP interaction rate per nucleus is thus given by:

$$
R=\sigma \frac{\rho_{D}}{M_{D}}\langle v\rangle
$$

Knowing the interaction rate of particles in a detector can help us set limits on the detection of WIMPs of various masses and with various spin-independent WIMP-nucleon cross sections. For a WIMP mass of $<10^{6} \mathrm{GeV}$, we can arrive at a WIMP-nucleon cross section of $\sim 10^{-35} \mathrm{~cm}^{2}$,implying an event rate of 1 event $/ \mathrm{kg}$ day in a detector[32][33].

Besides the interaction rate in the detectors, we need to calculate the recoil energy spectra in the detectors produced from the elastic scatter of a WIMP off a target nucleus. The recoil energy spectra can be determined as a function of WIMP masses using the nuclear form factor correction. Such a correction is made when the momentum transfer is such that the wavelength is no longer large compared to the nuclear radius and the effective interaction cross section begins to fall with increasing momentum transfer. The Helm form factor is given by 

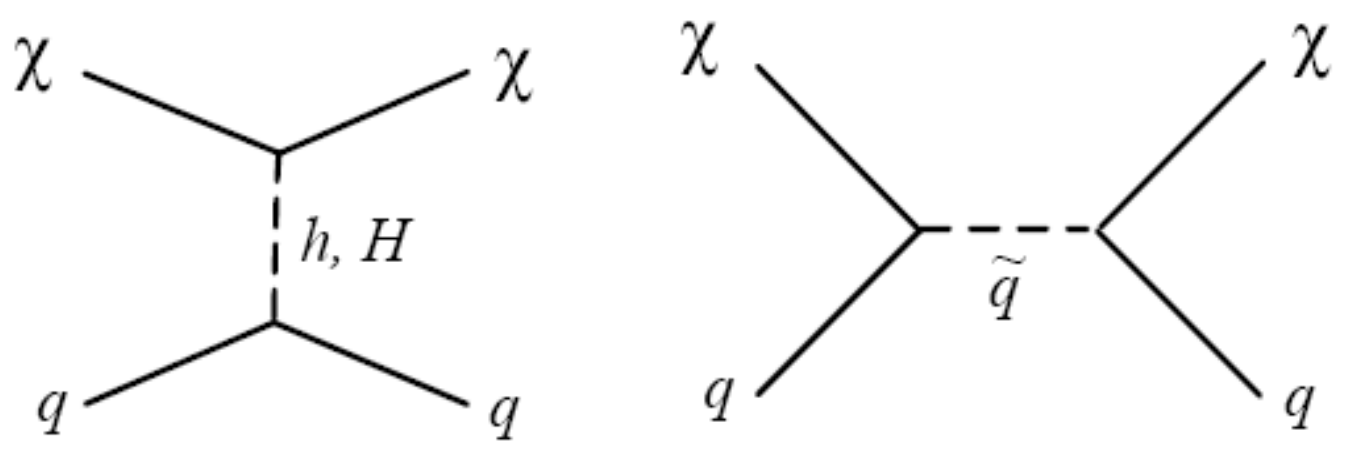

Figure 2.5: Representation of the neutralino-nucleus interactions at the neutralinoquark scale (taken from [28])

$$
F\left(q r_{n)}=3 \frac{j\left(q r_{n}\right)}{\left(q r_{n}\right)} \times e_{0}^{-(q s)^{2} / 2}\right.
$$

where $\mathrm{q}$ is the momentum transfer, $\mathrm{r}_{n}$ is the effective nuclear radius, $\mathrm{j}$ is the Bessel function and $\mathrm{s}=0.9 \mathrm{fm}[33]$. We take into account the integration of the velocity distribution and the form factor when computing the WIMP rate and spectrum.

From the above analysis, we can deduce that the interaction cross section increases with $\mathrm{A}^{2}$, where $\mathrm{A}=$ atomic weight of the target nucleus. This suggests that heavier nuclei like germanium make good target nuclei in direct detection experiments. For a germanium nucleus, we can determine that an elastic scatter of a WIMP of mass $100 \mathrm{GeV}$ would give rise to a mean recoil energy of $20 \mathrm{keV}$ and an event rate of less than 1 event per day for one kilogram of germanium.

\section{Inelastic scattering}

WIMPs scatter inelastically with the nucleus or the electrons of a target nucleus. The excited electron, or the nucleus as the case may be, that results from such a scatter de-excites emitting a decay photon which may be detected. 
Direct detection experiments rely on the scintillation and ionization or ionization and heat signals produced in a detector material due to a particle interaction. The CDMS search for WIMPs is explained in Section 2.7. Following is a brief look at some of the other ongoing direct detection WIMP-searches worldwide:

\section{Edelweiss}

The Edelweiss[34] experiment looks for WIMPs by measuring the charge and heat signals produced by the recoil of the dark matter particles off a germanium nucleus. The detectors are cylindrical-shaped germanium crystals of $70 \mathrm{~mm}$ diameter, $20 \mathrm{~mm}$ thickness and weighing $320 \mathrm{~g}$. The phonon or the heat signal is

measured using Neutron Transmutation Doped (NTD) thermistors that measure the rise in temperature of the crystal following a particle interaction. Electrodes on the top and bottom surface of the crystal record the charge signal produced by the production of electron-hole pairs as a result of a particle interaction. The information provided by the simultaneous measurement of the heat and charge produced in an interaction is used to discriminate between background and signal events. The experiment is located deep underground in the Laboratoire Souterrain de Moudane in the French Alps at a depth of 4800 m.w.e. Till the release of the CDMS data from the first experimental run at Soudan, the best limits on WIMP detection were recorded by Edelweiss from a $11.7 \mathrm{~kg}$-day exposure over data taken in 2000 and 2002.

\section{DAMA}

The only WIMP-search that claims to have seen a dark matter signal, the DAMA (DArk MAtter) experiment[35] studies the scintillation produced in a NaI crystal to determine the energy of a possible WIMP interaction with matter. The fiducial mass consists of nine NaI crystals making up a total of $100 \mathrm{~kg}$ mass. The experiment relies on scintillation in the detector material to record the energy of an interaction. The recoiling particle excites electrons to higher energy levels from 
which they de-excite emitting a photon that can be detected using photomultiplier tubes. Background rejection is done through pulse shape discrimination but this method of discrimination is not very effective for low energies. As the experiment can be scaled to large exposures quite easily, such detectors may be used to record the annual variation in the WIMP-nucleon scattering rate due to the Earth's movement through the galactic halo. DAMA claims to have seen an WIMP annual modulation signal that could correspond to a WIMP mass of $52 \mathrm{GeV} / \mathrm{c}^{2}$ and a spin independent nucleon cross-section of $7.2 \times 10^{-42} \mathrm{~cm}^{2}$. The claim has, however, been disputed by the non-observance of WIMPs by experiments such as CDMS[36][37] and Edelweiss[34].

\section{ZEPLIN}

The ZEPLIN[38] experiment records the scintillation and ionization produced from a particle interaction in a volume of liquid Xenon. An electric field applied across the Xe volume allows for an event-wise discrimination by suppressing the recombination of the ionized charge carriers. This gives rises to a prompt and a delayed signal. The relative sizes of the two signals help discriminate between electron and nuclear-recoils. Like the NaI detectors, the Xenon detectors also can be scaled easily to higher exposures, allowing for WIMP-searches to probe WIMP parameter space of cross-sections of $10^{-45} \mathrm{~cm}^{2}$.

\subsubsection{Indirect detection}

Another method adopted in searching for WIMPs is looking for by-products of WIMP-WIMP annihilations that occur either in the Sun or galactic halos. It was shown in Section 2.5.1 that the annihilation rate depends on the relic density of the species. As the neutralino is its own anti-particle, the annihilation rate of this form of dark matter depends on the square of the WIMP relic density. It is believed that WIMPs cluster gravitationally in dark matter halos about galaxies. WIMPs may 
also be trapped in stars and planets. If the annihilation rate is proportional to the square of the relic density, this can lead to a detectable signal of the by-products of the annihilation. The by-products are of the following types:

- WIMP annihilation in the core of the Sun or in the center of the Earth produce experimentally detectable high-energy (10 -1000 GeV) neutrinos. Such neutrinos are detected using Earth-based neutrino telescope that converts the neutrino into a charged lepton by virtue of a charged-current interaction in the material such as rock or ice that surrounds the detecting assembly[39][43].

- Annihilation in galactic halos give rise to gamma rays or rare cosmic rays such as positrons and anti-protons. The decay of the primary annihilation products give rise to a cascade of particles that include deuterons, positrons and gamma rays. The positron flux is sought to be detected by various space based or balloon based experiments[40]. The gamma ray flux may be detected by ground-based atmospheric Cherenkov radiation telescopes or space-based detectors[41]. The detection process involving searching for a line corresponding to the neutralino mass in the gamma ray spectrum[42].

\subsection{The CDMS approach}

The Cryogenic Dark Matter Search experiment employs direct detection techniques to search for WIMPs. It records the elastic scattering of WIMPs on target germanium and silicon nuclei by measuring the recoil energy $E_{r}$ of the nuclei. The detectors used are crystals of semiconductor material such as germanium and silicon. Since the recoil energies of interests are in the 10 -100 keV range, the detecting assembly needs to be maintained at cryogenic temperatures to ensure that the signal we seek is not masked by thermal vibrations of atoms and molecules seen at room temperatures. As the interaction rate for WIMPs is of the order of 1 event/kg-day while the self-same detectors can experience up to 4 million interactions per kg-day 
due to backgrounds arising from cosmic rays and natural radioactivity, the detectors are operated deep underground in a well-shielded environment. This acts to considerably reduce the backgrounds incident on the detecting assembly. Chapter III gives the basics of the CDMS search for WIMPs. 


\section{References}

[1] C. L. Bennett, M. Halpern et al, Astrophys. J. Suppl. 148, 1 (2003).

[2] G. Huey, R. H. Cyburt and B. D. Wandelt, astro-ph/0307080 (2003).

[3] P.J.E. Peebles, Principles of Physical Cosmology. Princeton University Press (1993).

[4] J.A. Peacock, Cosmological Physics, Cambridge University Press (1999).

[5] D. N. Spergel et al, Astrophys.J.Suppl., 148, 175 (2003).

[6] C.B. Netterfield et al., Astrophys.J., 571, 604 (2002).

[7] N.W. Halverson et al., Astrophys. J., 568, 38 (2002).

[8] R. Stompor et al., Astrophys. J., 561, 7 (2002).

[9] F. Zwicky, Helv. Phys. Acta, 6, 124 (1933)

[10] S. Smith, Astrophys J., 83, 23 (1936)

[11] J. H. Oort, Bull. Astron. Inst. Neth. 6, 249 (1932)

[12] V. C. Rubin and W. K. Ford, Ap. J., 159, 379 (1970).

[13] J. P. Ostriker and P. J. E. Peebles, Ap. J., 186, 467 (1973).

[14] E. Battaner and E. Florido, Fund. Cosmic Physics, 21, 1 (2000). 
[15] M. Persic, P. Salucci, and F. Stel, Mon. Not. Roy. Astron. Soc. 281, 27 (1996).

[16] Davis, D. S., and White, R. E., III., Astrophys. J., 470, 35 (1996).

[17] M. Loewenstein and R. E. White, Astrophys. J., 518, 50 (1999).

[18] N. Bahcall et al, Astrophys. J., 447, 81 (1995).

[19] R.G. Carlberg et al., Astrophys. J., 462, 32 (1996).

[20] A.E. Evrard et al, Astrophys. J., 469, 494507 (1996).

[21] I. Smail et al. Astrophys. J., 479, 70 (1996).

[22] D. Tytler et al., Physica Scripta, T85, 1231 (2000).

[23] S. Burles et al. Astrophys. J., 483, 778 (1997).

[24] O. Elgaroy et al, Phys. Rev. Lett., 89061301 (2002).

[25] E. P. S. Shellard and R. A. Battye, astro-ph/9802216.

[26] B.W. Lee and S. Weinberg, Phys. Rev. Letts., 39, 165 (1977).

[27] http://pupgg.princeton.edu/ martin/Ross_Nathaniel_JP.pdf

[28] J. Feng, hep-ph/0405215 (2004).

[29] G. Jungman et al, Physics Reports, 267, 195373 (1996).

[30] H. Goldberg, Phys. Rev. Letts., 50, 1419 (1983).

[31] J. R. Ellis et al, Nucl. Phys. B, 238, 453 (1984).

[32] M. W. Goodman and E. Witten, Phys. Rev. D 31, 3059 (1985).

[33] J.D. Lewin and P. F. Smith, 6Astropart. Phys., 6, 87 (1996).

[34] A. Benoit et al., Phys. Lett. B 545, 43 (2002). 
[35] R. Bernabei et al., Phys. Lett. B 480, 23 (2000).

[36] D. S. Akerib et al., Phys. Rev. D, 68, 082002 (2003).

[37] D. S. Akerib et al., astro-ph/0405033 (2004).

[38] S. Hart, Nucl. Phys. B Proc. Suppl. 110, 91 (2002).

[39] J. Ahrens et al., Phys. Rev. D 66, 032006 (2002).

[40] S. W. Barwick et al., Phys. Rev. Lett. 75, 390 (1995).

[41] L. Bergstrom, J. Edsjo and P. Ullio, Astropart.Phys. 9, 137 (1998).

[42] V. S. Berezinsky, A. Bottino and V. de Alfaro, Phys. Lett. B 274, 122 (1992).

[43] A. Habig et al., hep-ex/0106024.

[44] E. Corbelli and P. Salucci, MNRAS, 311, 441 (2000).

[45] http://www.astrographics.com/GalleryPrintsIndex/GP0017.html.

[46] A. C. Fabian and S. W. Allen, Proc. of the XXI Texas Symposium on Relativistic Astrophysics, Dec. 9-13 (2002). 


\section{Chapter 3}

\section{The CDMS Search for Dark Matter}

In July 2001, four germanium and two silicon crystals, instrumented for dark matter detection, went ultra-cold to temperatures of around $40 \mathrm{mK}$ and a new search for dark matter began at the Stanford Underground Facility.

The period of my graduate work overlapped with two CDMS WIMPsearches. In this chapter, I will discuss particulars of the first of these two runs conducted at a shallow site at the Hansen Experimental Physics Laboratory on the Stanford University campus. I will describe the detectors used, the shielding necessary to reduce unwanted backgrounds and the data acquisition and analysis techniques adopted.

The analysis of the data emerging from Run 21, as it has been so called, has been the subject of the theses of Tarek Saab[1] and Don Driscoll[2]. My work was geared towards simulating the neutron backgrounds seen at the shallow site and providing inputs to the background-reduction techniques necessary to reduce a data set of over 6 million low-background events to a small subset of WIMP-candidate events. 


\subsection{The ZIP detector}

The CDMS WIMP-search is conducted using Z-sensitive Ionization and Phonon Detectors(ZIPs). Figure 3.1 shows a typical ZIP detector which consists of $1 \mathrm{~cm}$ thick, $7.62 \mathrm{~cm}$ in diameter cylindrical crystals of germanium and silicon, weighing $250 \mathrm{~g}$ (for germanium) and $100 \mathrm{~g}$ (for silicon). Operated at cryogenic temperatures of $\leq 40 \mathrm{mK}$, these detectors are capable of particle identification on an eventby-event basis. A typical WIMP-search run may see over 6-7 million events, the overwhelming number of which arise from gamma, beta and neutron backgrounds incident on the detector. By employing such event-by-event discrimination techniques, the ZIP detectors are able to sift the signal from the background and allow the data analysis to reduce the total event population in a dark matter run to a WIMP-candidate data set.

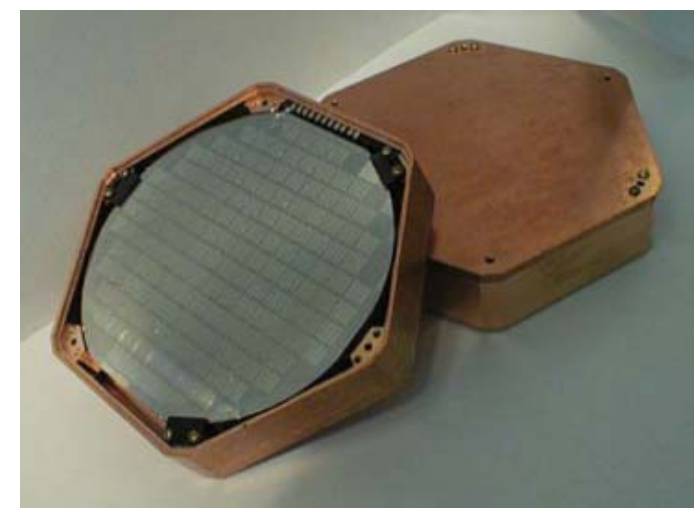

Figure 3.1: A Z-sensitive Ionization and Phonon Detector

\subsubsection{Event-by-event discrimination}

Crucial to the discrimination process is the nature of the recoil of the particle incident on the detector. As mentioned in Section 3.1, the detection relies on the elastic scattering of an incident particle from the germanium and silicon atoms 
that make up the detectors. Depending on the kind of interactions, the particles recoil either off the electrons or the nuclei of the germanium (or silicon) atoms. Photons and charged particles such as electrons and alphas undergo electromagnetic interactions. Such particles recoil off the electrons of the atoms of the detectors. Neutrons and WIMPs have nuclear or weak interactions which cause them to recoil off the nuclei of the germanium or silicon atoms. As such, but for the case of the neutrons, almost all backgrounds show electrons recoils as opposed to nuclearrecoils by possible WIMPs. The event-by-event discrimination capability of the ZIP detector consists essentially of distinguishing between the electron and nuclearrecoils seen by the dark matter detectors.

\subsubsection{Two-fold signature}

The ZIP's discrimination capability relies on the two-fold signature of a recoil of a particle from the atoms of the detector. The following process occurs when a particle interacts in a ZIP detector:

- The energy deposited in the interaction is used to generate electron-hole pairs in the crystal. If an electric field is applied across the crystal, the charge carriers drift to the opposite surfaces of the crystals giving rise to an ionization or charge signal.

- The particle interaction disturbs the crystalline lattice, the models of oscillation of which are defined as phonons. The deposited energy thus appears as a spectrum of high-energy $(\mathrm{THz})$ athermal phonons.

As the charge carriers drift across the surface of the crystal, they disturb the lattice giving rise to what are known as the Neganov-Luke phonons [3][4]. The charge bias applied across the crystal determines the work done in producing this second population of phonons. The total phonon energy is thus the sum of the 
athermal phonon energy that results from the original recoil and the energy of the Luke phonons created as a consequence of the voltage bias. This is given by:

$$
E_{\text {phon }}=E_{\text {recoil }}+e N_{e-h} V
$$

where $\mathrm{E}_{\text {phon }}$ is the measured phonon energy, $\mathrm{N}_{e-h}$ is the number of electron-hole pairs, $\mathrm{V}$ is the voltage bias and $\mathrm{E}_{\text {recoil }}$ is the phonon energy deposited in the recoil. The energy deposited in the phonon system due to the Neganov-Luke effect is the product of the charge $Q=e N_{e-h}$ produced in the initial interaction and the voltage bias $\mathrm{V}$ applied across the crystal.

$$
E_{\text {phon }}=E_{\text {recoil }}+\frac{Q V}{3.0(3.8)}
$$

where $3.0 \mathrm{eV}$ is the energy required to create an electron-hole pair in germanium while $3.82 \mathrm{eV}$ is the corresponding energy for silicon[5].

In the case of electron-recoil events, almost $70 \%$ of the energy is deposited as phonons. For low-energy nuclear-recoil events, the proportion is even larger with up to $90 \%$ energy being converted into phonons for a $10 \mathrm{keV}$ particle interaction[6]. Phonons thus constitute a major part of the energy deposited.

The number of electron-hole pairs produced due to a recoil depends on the nature of the interaction. The energy fraction used to liberate electron-hole pairs is greater in the case of an electron-recoil as compared to a nuclear-recoil event. A simultaneous measurement of the charge and phonon energy deposited in an interaction provides a means of discriminating against the particles causing the recoils. The discrimination parameter is defined as the Yield

$$
Y=\frac{Q}{E_{\text {recoil }}}
$$




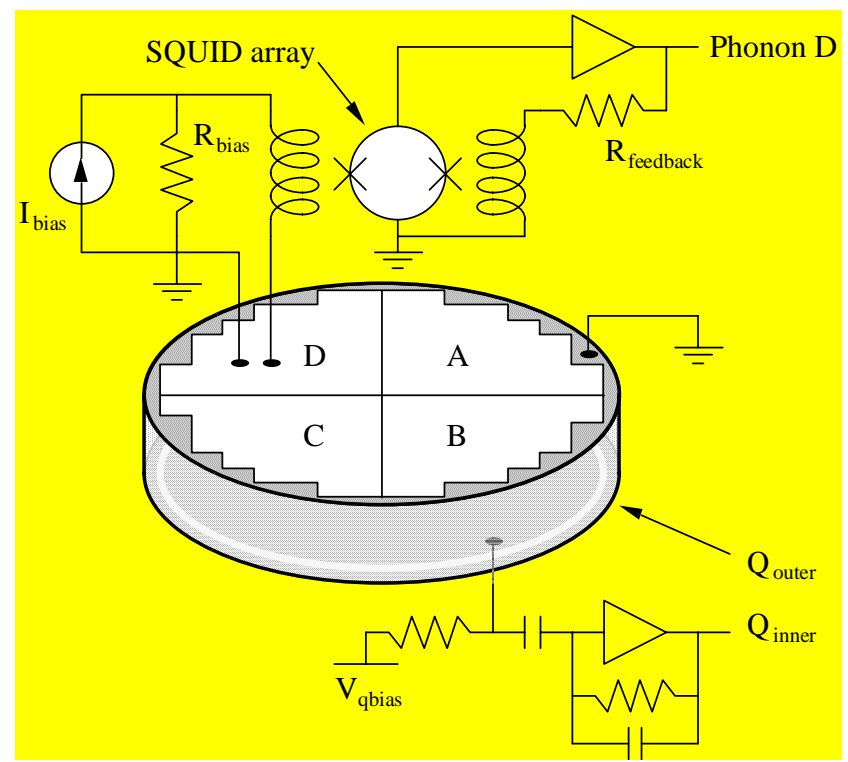

Figure 3.2: The two-fold measurement of the ionization or charge signal and the phonon or heat signal produced by a particle interaction in the ZIP detectors. The top half of the diagram shows the measurement of the phonon signal while a simple circuit to measure the charge signal is shown at the bottom of the page.

which gives the ratio of the charge to phonon energy produced in an interaction.

We now look at the instrumentation of the detector for the simultaneous measurement of the charge and phonon energy deposited due to a particle interaction. Figure 3.2 shows a schematic of the measuring circuit for the two energy channels.

\subsubsection{The Charge Channel}

The ZIP detector has two charge electrodes instrumented on it - an inner disk which covers $85 \%$ of the detector surface and an outer guard ring. A $1 \mathrm{~mm}$ gap separates the inner and outer charge sensors. Events occurring in the outer electrode are generally excluded from the dark matter analysis as they suffer from 
poor charge collection, allowing for low-yield electron-recoil events to be mistaken for nuclear-recoils. The two charge sensors are covered by a grid of $2 \mu \mathrm{m}$ aluminium lines spaced $20 \mu m$ apart[6].

When a recoil event occurs, electron-hole pairs are created. The energy required to create a electron-hole pair in germanium is $3 \mathrm{eV}$ while it is $3.82 \mathrm{eV}$ for silicon[7]. The number of electron-hole pairs created is thus given by:

$$
N_{e-h}=\frac{E_{r}}{3.0(3.8)}=\frac{Q}{1.6 \times 10^{-19}}
$$

A charge bias applied across the crystal causes the charge carriers to drift to their respective electrodes. The circuit produces voltage pulses such that the pulse height depends linearly on the energy of the recoil.

\section{Neutralization}

Poor charge collection can cause electron-recoil events to be mistaken for nuclear-recoil events. Charge carriers trapped in shallow impurity sites in the bulk of the crystal lead to poor charge collection. This problem is tackled by exposing the detectors to light from LEDs in a process called neutralization. Photons from the LED produce electron-hole pairs throughout the volume of the detector. These charge carriers fill the impurity traps and neutralize them, thus preventing charge carriers produced in a recoil event from being trapped by these sites. The LED baking is undertaken soon after cool-down of the detectors to temperatures below $1 \mathrm{~K}$ before data taking starts. LED flashing of about a minute long duration is done every few hours during normal data taking.

\section{Surface Effect}

Recoils which occur within a few $\mu m$ of the surface also suffer from poor charge collection. This surface effect, as it is so called, occurs because the charge 
carriers produced diffuse back against the electric field into the similarly charged electrode. A thin $40 \mathrm{~nm}$ layer of amorphous silicon, which has a larger band gap as compared to silicon and germanium, is deposited between the bulk of the crystal and the electrodes to minimize the diffusion of charge carriers into the electrode having the same charge [8].

\section{Choice of ionization-bias voltage}

The choice of the ionization-bias voltage to be applied is an important consideration for data taking. We noted that events that occur near the surface of the detector suffer from poor charge collection which can cause them to be mistaken for nuclear-recoil events. Increasing the charge-bias voltage causes an increase in the potential barrier against which charge carriers must diffuse to reach the electrode having the same charge, and thus reduces the surface effect. On the other hand, an increase in the bias voltage leads to an increase in the noise seen in the ionization and phonon channels and reduces the detector's capability of rejecting electron-recoils occurring in the bulk of the material. The choice of the charge bias, thus, depends on optimal discrimination of surface vis-a-vis bulk electron-recoils.

\subsubsection{The Phonon Channel}

One side of the ZIP detector is instrumented with the charge electrodes as described in Section 3.2.3. The other side of the crystal has Aluminium and Tungsten films deposited on it to form the phonon sensors. The surface is divided in four quadrants to form four separate phonon channels. Each quadrant has 888 tungsten meanders connected in parallel where each meander has dimensions of the order of $250 \mu \mathrm{m} \times 1 \mu \mathrm{m} \times 40 \mathrm{~nm}[9]$. Each meander is connected to Aluminium fins which absorb the phonon energy produced in a particle interaction and channel it into the tungsten sensors. 


\section{Transition Edge Sensor}

In its most basic form the ZIP detector behaves like a low temperature microcalorimeter consisting of a superconducting tungsten thin film deposited on a silicon or germanium substrate. The tungsten thin films have a sharp superconducting to normal transition in the range between $70 \mathrm{mK}$ and $130 \mathrm{mK}$ and are biased with a $1 \mathrm{mK}$ wide transition at about $70 \mathrm{mK}[9]$.

When particle interactions take place in the germanium or silicon crystal, the resulting energy is deposited as high-energy optical phonons, electron hole pairs and lattice damage, with the energy partition being dictated by the type of interaction and energy scales involved[6]. Phonons produced in the interactions propagate to the surface of the crystal where they are absorbed by the tungsten film. As the superconductor is biased near its transition temperature, it contains thermally excited quasiparticles that interact with the incoming athermal phonons and cause them to thermalize. This raises the temperature of the electrons in the superconductor.

The choice of tungsten as the superconducting element has been dictated by the very low transition temperature (typically $\sim 15.5 \mathrm{mK}$ ) the material can attain in the bulk state[10]. Temperature changes appear as resistive variations that are measured by SQUID-based electronics. These resistive variations are then turned back into a measure of the deposited energies, locations and arrival times of the incident particles.

\section{Electrothermal feedback}

To tackle problems of transition non-linearity and limited dynamic range in the detector, the transition edge sensor uses the heat input as an electrothermal negative feedback (ETF) that maintains the temperature of the superconducting film constant within its transition[11]. In the ETF mode, the sensor consists of a superconducting tungsten thin film deposited in a silicon substrate held at a temperature 
much below the Tc. When the film is cooled, its resistance approaches zero leading to a rise in the Joule heating. When heat loss to the substrate becomes equal to the Joule heating, stable thermal equilibrium is established. The bias resistance is chosen by varying the bias voltage applied. When a particle interaction occurs, the energy is deposited in the film causing a change of temperature, and a consequent change in the Joule power dissipation that in turn affects the temperature. The deposited energy is thus removed by a reduction in the feedback Joule heating and the detector self-biases on its transition.

\section{Quasiparticle assisted Trapping}

As WIMPS are weakly interacting, scattering events associated with them are rare. Detecting a sufficient number of such events requires the microcalorimeter to have a large absorber mass. For the detector to have a large absorber mass and yet exhibit good position and energy resolution, a larger detector surface area is desired. To do this without increasing the heat capacity of the thin film, the quasiparticle trapping technology is employed in the detector. Parallel arrays of tungsten thin film segments are deposited on the crystal substrate. Around each of these segments are large pads of Aluminum connected to the sensor by short spurs of tungsten. When incident particles are scattered in the silicon substrate, phonon pulses are produced. This phonon energy is incident on the Aluminum pads producing quasiparticles that diffuse in the TES on time scales short as compared to the quasiparticle recombination times. When these quasiparticles diffuse into the tungsten film, they interact with the electrons in the tungsten and get thermalized. This raises the temperature of the film, giving rise to a signal that can be measured. The phonon channel measurement thus amounts to determining the amount of heat deposited in the tungsten electron system. 


\subsubsection{Rise Time as a discrimination parameter}

The energy produced in a particle interaction manifests itself as the height of the voltage pulse produced at the read-out stage. Section 3.1.1 explained how different sources of backgrounds produce different signatures in the ZIP detectors. Gammas, alphas and electrons give rise to electron-recoil events while neutrons and the hypothetical WIMP scatter off the nucleus of a target atom. As specified in Section 3.1.2, the charge signal produced due to a nuclear-recoil event is smaller (about one third) compared to that produced as a consequence of an electron-recoil. electron-recoil producing backgrounds thus manifest themselves as high-yield events in the detectors but for one particular case - electrons producing recoils close to the surface of the detector. As stated in Section 3.1.3, such events suffer from poor charge collection and can be mistaken for low-yield nuclear-recoil events. The rise time of the phonon pulse is used a means of discriminating against such events. Figure 3.3 shows how the rise time of the phonon pulse may be used as a means of discriminating against low-yield betas which can be mistaken for nuclear-recoil events. The two-stage analysis of the Run 21 data was based on a difference in the criteria for rejecting surface betas. While the first analysis employed a flat rise time cut, rejecting all events that had rise times $<12 \mu \mathrm{s}$ for the germanium detectors and $<6 \mu$ s for the silicon detectors[1], the combined analysis of the $3 \mathrm{~V}+6 \mathrm{~V}$ data used the maximum likelihood ratio analysis method[2] to discriminate against lowyield surface betas leaking into the signal region. The rise time analysis for the $3 \mathrm{~V}$ data was primarily done by Tarek Saab and Vuk Mandic and that of the 3V+6V by Don Driscoll. 


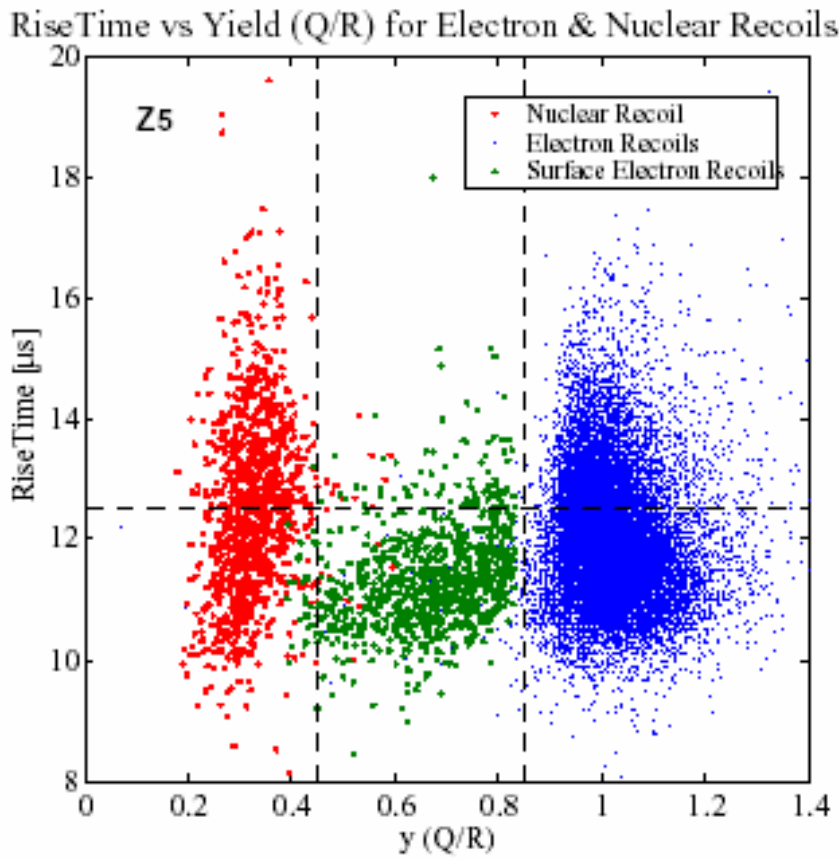

Figure 3.3: Rise time of the phonon pulse as a discrimination parameter. Low yield electron recoil events such as those arising from surface electrons have short rise times on account of their being surface events. Bulk nuclear recoil events which also have low yield have larger rise times. This parameter is thus used to reject surface beta leakage into the signal region (taken from [1]). 


\subsection{Run 21 at Stanford}

\subsubsection{Rationale for the Run}

With cryogenic-related problems delaying the move deeper down to the 2090 m.w.e Soudan Underground Mine in Northern Minnesota, the experiment had perforce to continue at the Stanford facility. An earlier run at the same site had established the existence of a limiting background produced by high-energy neutrons produced from cosmic-ray muons interactions in the rock of the experimental cavern[12].

Even so, it was expected that another run here would improve on our sensitivity to WIMP detection given that it would feature the full complement of the new design Z-sensitive Ionization and Phonon Detectors with their superior background discrimination capabilities. Besides, additional shielding in the form of an extra layer of polyethylene had been added to further contain the neutron background.

The "tower" of six ZIP detectors, four germanium and two silicon, to be used at Stanford would subsequently be moved to Soudan, allowing for Run 21 to serve as a dry run for the detector configuration. Another rationale for the run was to confirm the hypothesis that "punch-through" neutrons were the limiting neutron background seen at this site.

\subsubsection{Time line and structure of the Run}

From July 2001 to August 2002, six ZIP detectors took over 8.7 million events in the low-background data-taking mode to assemble a comprehensive WIMP-search data set. The experiment was conducted at the 17 m.w.e. Underground Facility at Stanford. 


\subsubsection{The Icebox and Dilution Refrigerator}

As explained in Section 2.7, a critical part of the CDMS WIMP-search is operating the ZIP detectors at milliKelvin temperatures so as not to allow thermal vibrations in atoms and molecules at room temperature to mask the recoil energies produced in a particle interaction. For the run at Stanford, a Kelvinox 400-S dilution refrigerator was used to cool the detectors to temperatures of $\sim 20 \mathrm{mK}$. The refrigerator is connected through a 'Cold stem' to the 'Icebox', that consists of, moving outward, a series of concentric cylindrical copper cans heat sunk to temperatures of $20 \mathrm{mK}, 50 \mathrm{mK}, 600 \mathrm{mK}, 4 \mathrm{~K}, 77 \mathrm{~K}$ and room temperature. The innermost can of the Icebox holds the 'Tower' of six detectors used for the WIMPsearch.

\subsection{Backgrounds seen at SUF}

At the 10.6-meter depth of the SUF facility, the integrated muon flux is reduced from the value of 180 muons $/ \mathrm{m}^{2} / \mathrm{s}$ at sea level to 44.4 muons $/ \mathrm{m}^{2} / \mathrm{s}[13]$. At this depth, the hadronic component of the cosmic rays is negligible while their muonic component is reduced by a factor of 4 from the surface value. Backgrounds arising from natural radioactivity and cosmic rays were of the order of 1 event $/ \mathrm{kg} / \mathrm{day}[14]$. The relatively large muon flux ensured that we needed good shielding to be able to discriminate against the various backgrounds incident on the detectors.

The principal backgrounds seen at the site included:

- Gamma rays resulting from natural radioactivity, radioactive contaminants in the detector and shielding material and those arising from the cosmic-ray muon flux seen at the experimental site[13]. Radioactive nuclides from the ${ }^{238} \mathrm{U}$ and ${ }^{232} \mathrm{Th}$ chains and the presence of ${ }^{40} \mathrm{~K}$ in the rock and the surrounding material give rise to photons. Radioactive nuclides are also produced in the atmosphere and in the materials of the detector and shielding from the 
interactions of cosmic-ray muons with the material. Photons can also arise as a consequence of contaminants in the detectors and the shield.

- Betas can arise from radio-contaminants on the surface of the detectors, presence of radon in the atmosphere as well as radioactive nuclides in the detector and shielding material. Of special interest are the class of electrons that scatter near the detector surface. The events that arise from such interactions suffer from poor charge collection and may be mistaken for nuclear-recoils. Gamma rays that scatter in a detector can knock off electrons from target atoms which in turn exhibit surface-scatters in neighboring detectors. We call this class of events 'ejectrons'. For the current run at SUF, surface electrons arising from radio-contaminants on the detector surfaces coupled with such ejectrons were a considerable source of background.

- Neutrons are produced from cosmic-ray muon interactions with the material of the rock and the shield and from the presence of radioactive nuclides in the rock and the surrounding material. This topic has been covered in great detail in Chapter IV.

As the expected interaction rate of WIMPs in our detectors is of the order of 1 event per kg-day, it is essential to have a good shielding assembly to reject most backgrounds. Figure 3.4 shows the shield used for the WIMP-search run at SUF. Moving inwards, the components of the shield are:

- A scintillator muon veto that rejects beta, gamma and neutrons produced by cosmic-ray muon interactions with $99.99 \%$ efficiency[1].

- A $15 \mathrm{~cm}$ outer lead shield that acts like a gamma attenuator and reduces the photon flux by a factor of 1000 .

- A $25 \mathrm{~cm}$ outer polyethylene shield that reduces the muon-induced neutron flux produced in the outer rock and the surroundings by a factor of 100 . 


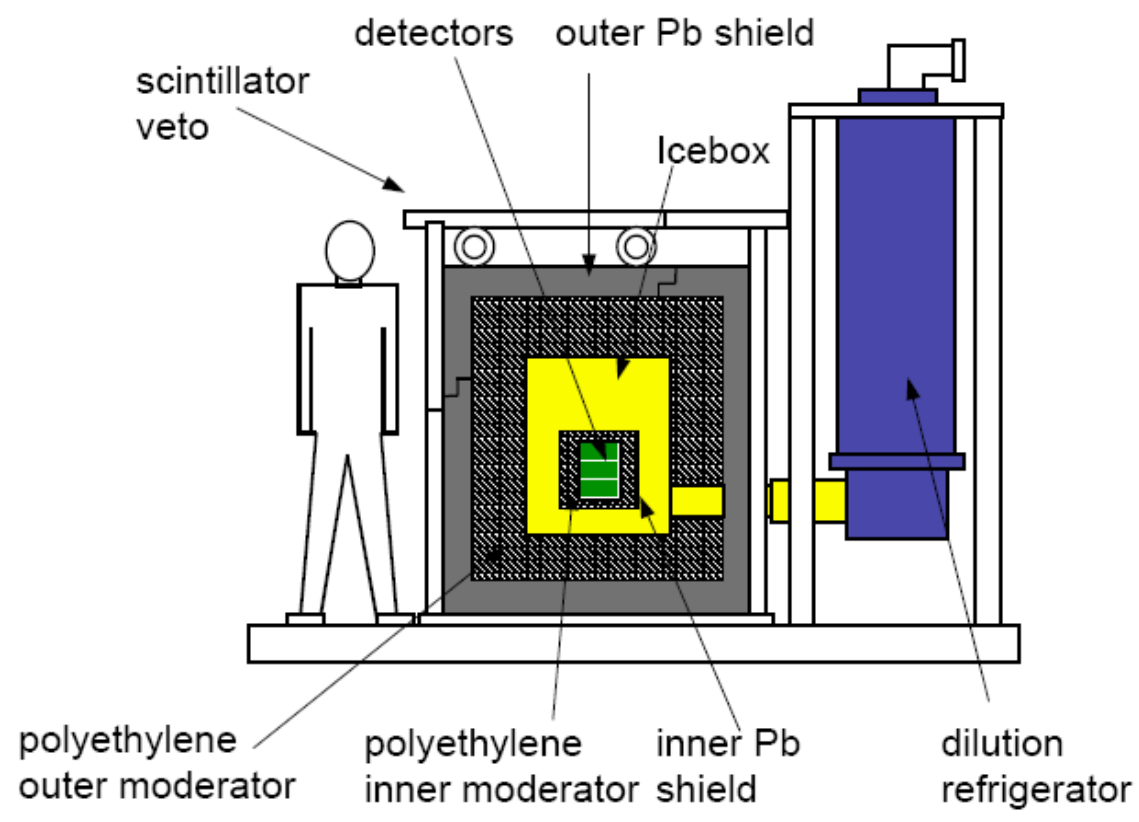

Figure 3.4: Icebox and shield for Run21 at the Stanford Underground Facility. Shown is the dilution refreigerator and the icebox with the veto, lead and polyethylene layers.

- A $43 \mathrm{~kg}$ inner lead layer placed inside the icebox that reduces the photon flux by a factor of 10 .

- $11 \mathrm{~kg}$ of additional polyethylene placed inside the inner lead shield that suppresses the neutron flux produced by muon interactions in the material of the shield by a factor of 2.5. This layer of shielding was added following the results of the previous WIMP-search at SUF which indicated the presence of a limiting neutron background at the site[12].

The shielding reduces the incident gamma rate to $\sim 1$ event $/ \mathrm{keV} / \mathrm{kg} /$ day. It also reduced the neutron rate to 0.01 event $/ \mathrm{keV} / \mathrm{kg} /$ day in the energy range of 5 -100 keV[1]. 


\subsection{First analysis of the $3 \mathrm{~V}$ data}

For Run 21, the WIMP-search data was taken at two ionization bias voltages - $3 \mathrm{~V}$ and $6 \mathrm{~V}$. At $3 \mathrm{~V}$ bias, 65.8 live days of WIMP-search data were taken from December 2001 to April 2002 which reduced to $28.3 \mathrm{~kg}$-days after cuts. The first

analysis of the $3 \mathrm{~V}$ data has been covered in detail in [1]. The analysis indicated that the new-design ZIP detectors were capable of gamma rejection greater than $99.99 \%$ for the energy range of 5-100 keV and rejected bulk electrons at greater than $99 \%$ for energies above $10 \mathrm{keV}$. The run also confirmed the presence of a limiting background from high-energy neutrons emerging from the rock and punching through the shield to produce secondaries that give rise to recoil events in our detectors.

The WIMP-search data was taken over a period of eight months. Several calibration runs with test sources such as ${ }^{137} \mathrm{Cs},{ }^{60} \mathrm{Co}$ and ${ }^{252} \mathrm{Cf}$ were carried out interspersed with the WIMP-search data taking. The ${ }^{137} \mathrm{Cs}$ source was used to ensure that the detectors were neutralized prior to data taking. This is done by exposing the detectors to an external monochromatic source of radiation and verifying that the shape and position of the $662 \mathrm{keV}$ line in the ${ }^{137} \mathrm{Cs}$ spectrum are as expected in the ionization spectra according to Monte Carlo simulations. This, in turn, allows us to determine the value of the calibration constants used to convert pulse height to an energy representation and set the energy calibration scale for both the charge and the phonon channels[15].

\subsubsection{Data cuts applied-}

The over six million events during a WIMP-search were reduced to a subset of 20 WIMP-candidate events[15] using a series of data cuts. The cuts applied were as follows:

- Data quality cuts: Cuts removing pile-up events, bad data, events occurring during periods of electronic glitches and those associated with variations in the charge and phonon baselines. 
- Cuts to define the threshold energies for the charge and phonon channels. The charge threshold cut ensure that events with no charge signal are not mistaken for low-yield events by selecting events with charge pulses large enough to be inconsistent with noise fluctuations. For Run 21, the charge threshold was set at $1.5 \mathrm{keV}[1]$. The phonon threshold was set at $5 \mathrm{keV}$ for all detectors except Z1 which had a threshold of $20 \mathrm{keV}$ due to its poor energy resolution.

- The fiducial-volume cut rejecting events that fall in the outer guard electrode of the detectors. Such events suffer from poor charge collection and are not considered in the dark matter analysis. For the first analysis of the Run 21 data, the fiducial-volume cut passed all events with:

$$
0.8<\frac{q_{i}-q_{o}}{q_{i}+q_{o}}<1.2
$$

- The nuclear-recoil band cut selecting low-yield events that may be characterized to fall in the signal region. The band is defined from Gaussian fits to the histograms of the yield distribution over various energy bins for neutron calibration data taken with a ${ }^{252} \mathrm{Cf}$ source. Based on the fits, the mean and standard deviation of the mean of the nuclear-recoil band are found and the band defined, choosing events within $\pm 2 \sigma$ of this means value.

- The rise time cut which discriminates against low-yield electron-recoil events leaking into the signal events and being mistaken for nuclear-recoil events. As most of these low-yield electron-recoil events occur near the surface where there is poor charge collection, a cut on the rise time of the phonon pulse acts as a good discrimination parameter as explained in Section 3.1.5. The first analysis of the Run 21 data employed a flat rise time cut rejecting events that fell below $12 \mu \mathrm{s}$ in rise time for the germanium detectors and $6 \mu \mathrm{s}$ in rise time for the silicon detectors.

- The singles cut selects events which are characterized by the absence of a trigger in the phonon channels in all but one detector within $50 \mu s$ of an event trigger. 
- The multiples cut defines an event as a multiple if all detectors pass the data quality cuts and two or more detectors pass the phonon threshold and fiducial-volume cuts. For the case of nuclear-recoil candidates, two or more scatters should also fall in the signal band. The multiple cuts passes the rise time cut if at least one detector passes the rise time cut.

- A muon veto cut that selects all events in the detectors for which the time to the most recent trigger in the muon veto scintillator is more than $40 \mu \mathrm{s}$.

The WIMP-candidate data set comprises the subset of single-scatter nuclearrecoil events in the germanium detectors. On application of all the cuts specified above, we arrived at 20 single-scatter events in the germanium detectors Z1, Z2, Z3 and Z5. 2 single-scatter events were recorded in the silicon detector Z4. Z6, the other silicon detector, was not considered for the analysis as it had a history of ${ }^{14} \mathrm{C}$ contamination.

In Chapters V, we study the use of the data analysis to check the simulations of the neutron calibration and muon-coincident neutrons events in the low background data. Chapter VI uses the results of the simulations to estimate and subtract the neutron contribution to the signal.

\subsection{Maximum Likelihood Ratio Analysis of the extended Run 21 data}

The neutron subtraction method applied to the dark matter analysis provided ratios of single-scatter events in the germanium detectors to the number of multiple-scatters. This ratio, when applied to the number of multiples seen in the WIMP-search data, provides a means of estimating the neutron contribution to the single-scatter event population. It is therefore important to be able to get as good a measure of the number of multiples in the data so as to make the statistical 
subtraction as precise as possible. The first analysis of the Run 21 data was hampered by the inability to quantify the leakage of low-yield surface betas into the nearest-neighbor double-scatter population. While the population of triple-scatters and non-nearest-neighbor scatters showed good separation between the signal and background regions, this was not the case for the nearest-neighbor candidates.

The analysis of the Run 21 data taken at $3 \mathrm{~V}$ and $6 \mathrm{~V}$ bias saw the application of the maximum likelihood ratio analysis method to quantify the beta leakage into the signal region. The WIMP-search analysis using the method was done by Don Driscoll[2]. Instead of a flat cut in the rise time-yield plane as was the method for discrimination in the first case, here we seek to find the probability of an event arising from a beta based on an examination of the rise time of the phonon pulse, the yield and the recoil energy for an event. An explanation of the method has been detailed in Don Driscoll's thesis. I will give a brief explanation of the method used. A sample population of betas is selected from the nearest-neighbor double-scatter events in the gamma calibration. To this population, we fit a distribution function for the betas. To the single-scatter event population in the neutron calibration, a similar distribution function is fit for the neutrons. For the yield, recoil energy and rise time associated with each event, the relative probability that an event is a beta or a neutron is determined. A cut level is selected depending on the ratio of the probabilities which preferentially reject the betas.

For the extended analysis, the fiducial-volume cut was also redefined to accept events that were $5 \sigma$ above the noise in the inner charge electrode region and consistent with noise in the outer region[15]. This cut was more restrictive in that it did not accept events shared between the two electrodes. The fiducial-volume cut takes into account the energy dependence in the cross-talk between the inner and outer electrodes. This cross-talk is both detector and bias voltage dependent.

Based on this analysis, the $3 \mathrm{~V}+6 \mathrm{~V}$ data gave a WIMP-candidate data set of 19 single-scatter events in the three germanium detectors Z2, Z3 and Z5. Chapter $\mathrm{V}$ details how the cuts were applied to check the simulations of the neutron calibrations. In Chapter VI, we check the efficiencies of the cuts against a sample 
population of simulated neutrons and apply the efficiencies of the data to the simulations of the external neutron background to estimate, and subtract, the neutron contribution to the signal. 


\section{References}

[1] T. Saab, Ph.D. thesis, Stanford University (2002).

[2] D. D. Driscoll, Ph.D. Thesis, Case Western Reserve University (2004).

[3] P. Luke, J. Appl. Phys., 64, 6858 (1988).

[4] B. Neganov and V. Trofimov, Pis'ma v Zhurnal Experimental'noi $i$ Teoreticheskoi Fiziki, 28, 356 (1978).

[5] G.F. Knoll, Radiation Detection and Measurement., John Wiley and Sons, Inc., (1999).

[6] K. D. Irwin, Ph.D. Thesis, Department of Physics, Stanford University (1995).

[7] K.D. Irwin et al, Rev. Sci. Instrum., 66, 10 (1995).

[8] T. Shutt et al., Nucl. Instr. and Meth. A, 444, 340344 (2000).

[9] B Cabrera, Proc. of the VIII Intl. Workshop on Low Temp. Detectors, Dalfsen, The Netherlands (1999).

[10] T. Van Duzer, Principles of Superconducting Devices and Circuits, PrenticeHall, New Jersey, 1999.

[11] K.D. Irwin, Appl. Phys. Lett., 66, 1998 (1995).

[12] R. Abusaidi et al, Phys. Rev Lett., 84, 5695 (2000). 
[13] A. da Silva, Ph. D. Thesis, The University of British Columbia, (1996).

[14] D. Abrams et al., Phys. Rev. D 66, 122003 (2002).

[15] D. S. Akerib et al., Phys. Rev. D, 68, 082002 (2003).

[16] D.D. Driscoll, Run 21 Ebook Note 305 (2003). 


\section{Chapter 4}

\section{The Neutron Background and how it Limits Sensitivity to WIMP Detection}

A kilogram of germanium at sea level is expected to have less than one WIMP interaction per day. The same material experiences over 4 million interactions per day due to backgrounds arising from cosmic-ray muons, natural radioactivity and various sources of contamination. To allow for the signal we are seeking to be heard above this level of noise, it is essential to quantify and discriminate against the various backgrounds seen by the experiment.

The previous chapter showed how depth, good shielding and the excellent discrimination provided by ZIP detectors considerably reduce most backgrounds in the CDMS WIMP-search. It also indicated that while anti-coincidence shielding and the detector's rejection parameters work well against electromagnetic backgrounds such as $\alpha, \beta$ and $\gamma$ rays, they are not as effective in rejecting neutrons. For the latter, the solution lies in moving deeper underground.

This chapter examines the neutron backgrounds seen at the shallow and deep sites of the CDMS WIMP-search. It discusses the sources of neutron back- 
grounds, the physical processes involved in their production and the relative importance of the different processes at different depths.

As better shielding, higher levels of radio-purity and excellent discrimination against typical gamma and beta backgrounds allow dark matter searches to aim for sensitivities of $10^{-9}-10^{-10} \mathrm{pb}$ to the WIMP-nucleon cross-section for WIMP masses of $40-80 \mathrm{GeV} / \mathrm{c}^{2}$, the need to identify and suppress the neutron contribution becomes that much more critical.

\subsection{Sources of the Neutron Backgrounds}

The principal sources of neutron backgrounds at the two CDMS experimental sites underground are cosmic rays and natural radioactivity. Figure 4.1 shows a schematic of the main types of neutron backgrounds incident on the detecting assembly.

Neutrons from natural radioactivity result from $(\alpha, \mathrm{n})$ reactions induced by alpha decay in the uranium and thorium traces in the rock and the detector and shielding material. Spontaneous fission of ${ }^{238} \mathrm{U}$ and ${ }^{232} \mathrm{Th}$ in the rock and other materials in the vicinity of the detectors also contributes to this flux.

At underground sites, cosmic rays consist mainly of muons and neutrinos. As they traverse the Earth's crust, the muons undergo interactions that produce particles such as neutrons, protons, pions, $\gamma$-quanta and various radioactive isotopes in hadronic cascades and electromagnetic showers. The charged components of the cosmic rays can be rejected at high efficiency by an active veto surrounding the detectors. Not so the case with neutron secondaries produced by this muon flux.

Neutron production by muons underground can arise from $\mu^{-}$capture by a nucleus followed by the emission of a neutron or as a consequence of photoproduction by the inelastic-scattering of muons on nuclei. Neutrons may also be produced by hadrons in secondary nuclear-cascades or by photons in electromagnetic showers, initiated by virtual and real photons respectively. 


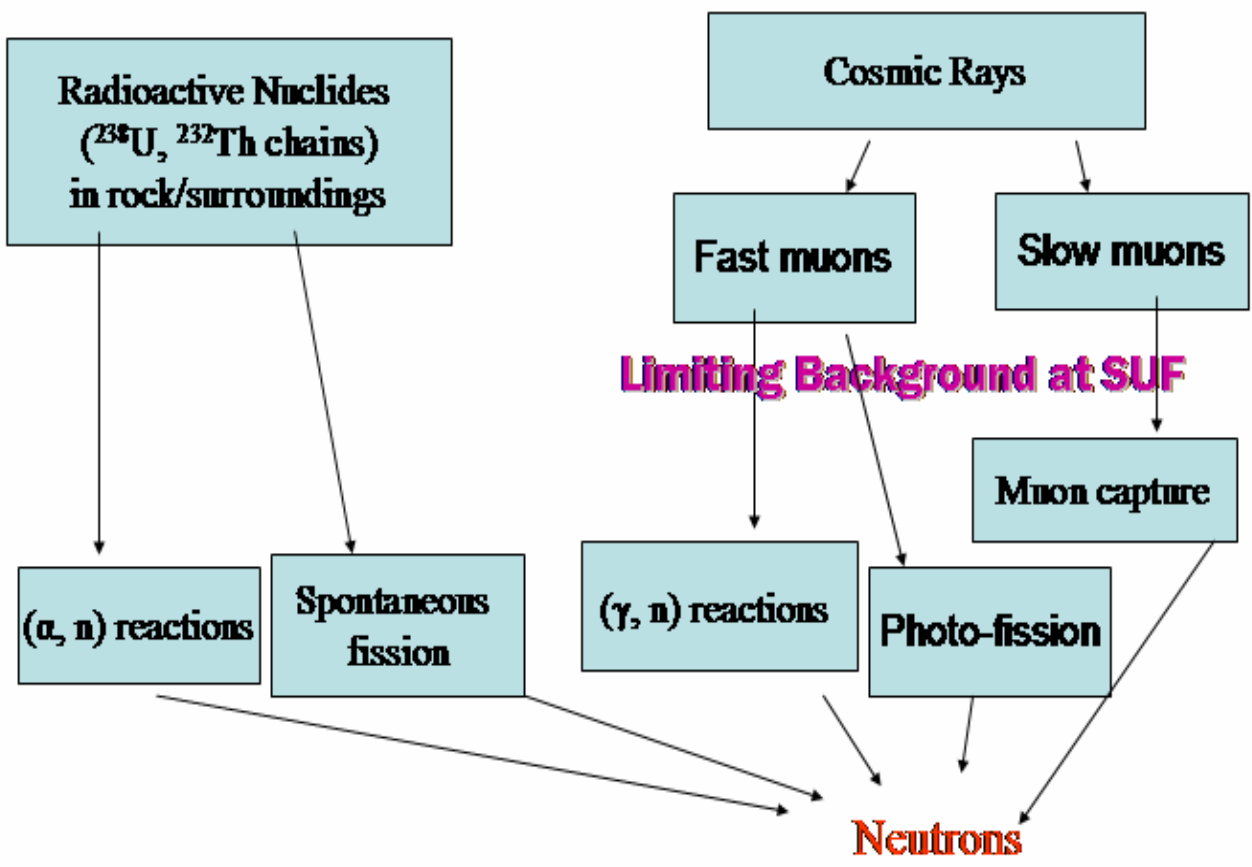

Figure 4.1: Neutron Backgrounds

While the neutrons arising from radioactivity and spontaneous fission have low energies (of the order of few $\mathrm{MeV}$ ), those generated from inelastic muon-nucleus scattering can have energies of up to $100 \mathrm{GeV}[1]$.

\subsubsection{Depth Dependence}

Depth plays an important role in determining the relative importance of the various neutron fluxes. The neutron yield from cosmic-ray muon interactions, in particular, is strongly related to depth given that a large overburden of rock can greatly suppress the muon, and hence the neutron, flux.

Very close to the surface, the hadronic component of the cosmic-ray muons is the principal source of the neutron flux. This contribution is depth- -dependent, reducing by a factor of 10 at a depth of 5 m.w.e. and by a factor of 1000 at depths 
of 10 m.w.e[2].

Neutron production from cosmic-ray muon interactions with the surrounding material is the main production mechanism at intermediate depths, as has been observed at the 17-m.w.e Stanford Underground Facility.

As the muon flux attenuates with depth, so does the rate of muon-induced neutrons. At depths greater than $3 \mathrm{~km}$. w.e., the rate falls to a factor of 1000 below the rate of neutrons from rock activity[3], making neutrons from radioactivity the main source of neutrons deep underground. Figure 4.2 illustrates the relative importance of the different neutron production processes in a material as a function of depth.

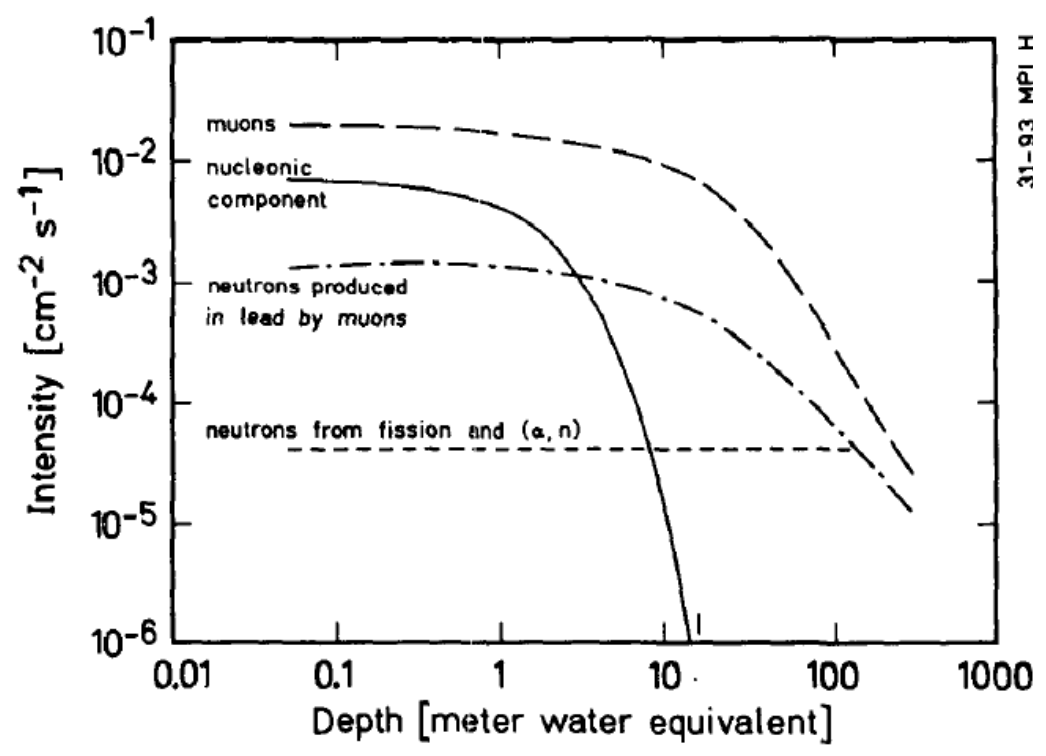

Figure 4.2: Flux of neutrons produced in lead as a function of depth. As can be seen, the nucleonic component falls off at low depths and becomes negligible at depths greater than 10 m.w.e. Muon-induced neutrons also decrease with depth as shown. Above 100 m.w.e neutrons produced from natural radioactivity become the dominant flux. (taken from [3]) 
However, even at deep sites, neutrons produced by muon interactions continue to be the troubling backgrounds as neutrons resulting from $(\alpha, \mathrm{n})$ and fission reactions tend to have energies of less than $10 \mathrm{MeV}$, allowing them to be easily suppressed by a layer of polyethylene shielding [3]. The muon-induced neutrons, having much higher energies, are able to travel greater distances away from the associated muon track, punching through the shield to produce secondary neutrons that give rise to nuclear-recoil events similar to WIMP signals in our detectors.

Figure 4.3 indicates the different interaction rates of neutrons in silicon and germanium, the two elements that constitute the detector material. The recoils produced due to neutrons have energies between $10-100 \mathrm{keV}$, the energy range of the WIMP signal of interest. While silicon has a higher interaction rate per kilogram for neutrons, germanium is more sensitive to WIMPs, allowing for means of materialwise discrimination of the signal seen in the two types of detectors. This difference in interaction rates of germanium and silicon for neutrons is used to make estimates of the neutron background in the background subtraction techniques explained in Chapter VI.
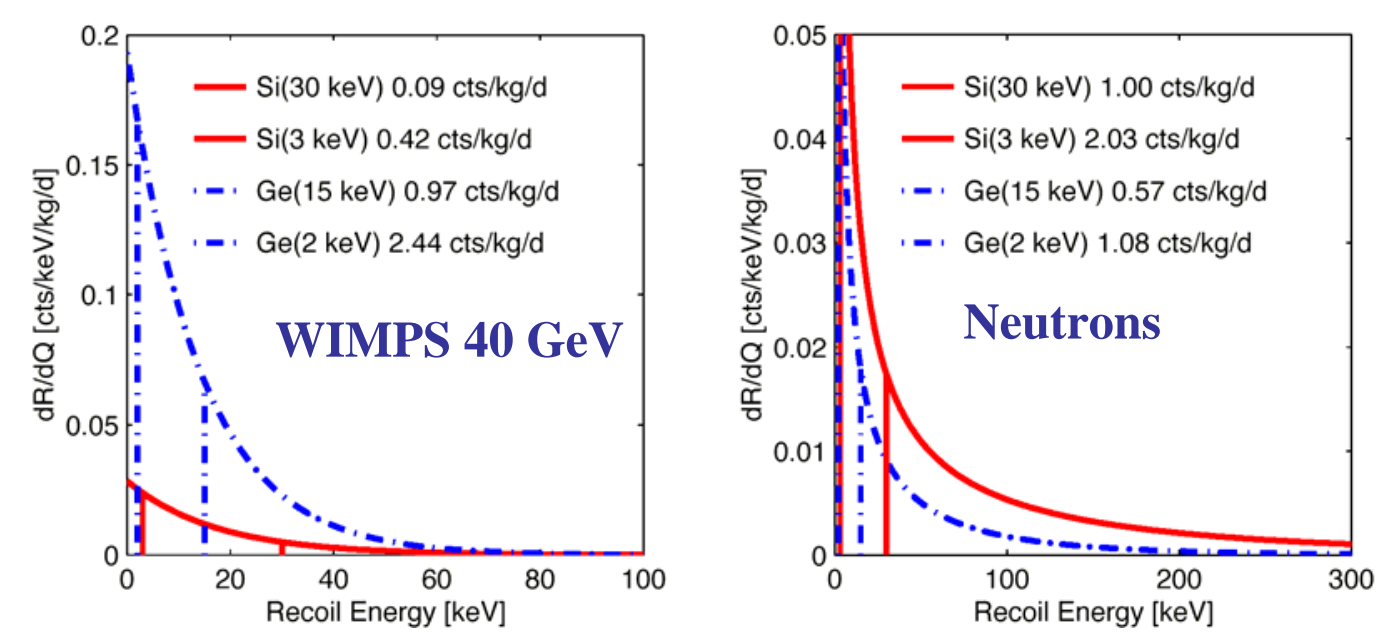

Figure 4.3: Interactions rate of neutrons and WIMPs in Silicon and Germanium (from B. Cabrera). 


\subsubsection{Neutrons from Natural Radioactivity of the Rock}

The natural activity of rocks is dominated by the ${ }^{238} \mathrm{U}$ and ${ }^{232} \mathrm{Th}$ decay chains and ${ }^{40} \mathrm{~K}$ which has a relative abundance of ${ }^{40} \mathrm{~K} / \mathrm{K}$ of $1.2 \times 10^{-4}[3]$. Alpha decay in the uranium and thorium chains lead to $(\alpha, \mathrm{n})$ reactions that produce neutrons. The alpha particles produced from these processes have energies less than 8.78 $\mathrm{MeV}$, too low to induce $(\alpha, \mathrm{n})$ reactions in ${ }^{16} \mathrm{O},{ }^{28} \mathrm{Si}$ and ${ }^{40} \mathrm{Ca}$ which together make up $79 \%$ of the Earth's crust. $(\alpha, \mathrm{n})$ reactions are induced in $\mathrm{Al}, \mathrm{Na}, \mathrm{Mg}$ and ${ }^{18} \mathrm{O}$ yielding about 30 neutrons/kg/day of rock, having energies of a few $\mathrm{MeV}[4]$.

The low-energy neutron flux also has a contribution from the spontaneous fission of ${ }^{238} \mathrm{U}$ and ${ }^{232} \mathrm{Th}$. The energy spectrum [5] of neutrons from this process is estimated as:

$$
d N(E)=\sqrt{E} e^{-E / 1.29} d E .
$$

where $\mathrm{E}$ is the energy in $\mathrm{MeV}$. The spectrum is a steeply falling exponential with a characteristic energy of $1.29 \mathrm{MeV}$. At the shallow site, the production rate is expected to be 4.1 neutrons $/ \mathrm{kg} /$ day in rock [6]. This flux, however, is easily suppressed by the use of passive hydrocarbon shielding.

Neutrons from radioactivity are also important at the 2090 m.w.e. Soudan Underground Facility. Here the cavern is made of a green rock of volcanic origin known as Ely Greenstone made principally of $50.6 \% \mathrm{SiO}_{2}, 15 \% \mathrm{Al}_{2} \mathrm{O}_{3}$ and 7 $9 \%$ of $\mathrm{CaO}, \mathrm{FeO}$ and $\mathrm{MgO}$, besides other compounds. Table 4.1, taken from [7], lists the concentration of the radioactive isotopes present in the rock. The neutron production rate due to $(\alpha, \mathrm{n})$ processes in the rock is estimated to be $(2.1 \pm 0.2) \mathrm{x}$ $10^{-8}$ neutrons/g/s and fission is expected to yield $2.7 \times 10^{-9}$ neutrons/g/s [7].

\subsubsection{Neutrons from Radioactivity in the Shield}

Neutrons may also be produced from $(\alpha, \mathrm{n})$ reactions and spontaneous fission in the traces of ${ }^{238} \mathrm{U}$ and ${ }^{232} \mathrm{Th}$ found in the material of the detectors and the shield. 


\begin{tabular}{|c|c|}
\hline Radioactive Isotopes & Concentration \\
\hline${ }^{238} \mathrm{U}$ & $0.17 \pm 0.06 \mathrm{in} \mathrm{ppm}$ \\
\hline${ }^{232} \mathrm{Th}$ & $0.89 \pm 0.2 \mathrm{in} \mathrm{ppm}$ \\
\hline${ }^{40} \mathrm{~K}$ & $0.79 \pm 0.04 \%$ \\
\hline
\end{tabular}

Table 4.1: Concentration of radioactive isotopes in the Soudan rock

Alpha particles produced from the decay of ${ }^{238} \mathrm{U}$ and ${ }^{232} \mathrm{Th}$ have energies too low to induce $(\alpha, \mathrm{n})$ reactions in the materials. Neutrons from such processes are produced from spontaneous fission. As neutrons produced in this manner have low energies and can easily be suppressed by passive shielding, only the contribution from the material of the shield within the polyethylene layer need be considered. Earlier studies for the shield configuration at the shallow site[8] estimate a detection of $<$ 1 neutron for a $27 \mathrm{~kg}$-days exposure.

With most dark matter searches achieving a high level of radio-purity in their shielding material, such backgrounds are expected to fall to a factor of 1000 below other neutron contributions[9] and may safely be neglected.

\subsection{Neutrons from Cosmic Ray Muons}

Neutrons arising from cosmic-ray muons are the other principal source of this flux underground. Unlike their radioactivity-induced counterparts, these may be fast neutrons with energies of the order of several hundreds of $\mathrm{MeV}$. Their rate depends on the muon flux seen at the site and consequently exhibits a dramatic reduction (of the order of $10^{3}-10^{6}$ from the ground level) as you move deeper underground.

Moving from the 17 m.w.e. shallow site to the 2090 m.w.e. Soudan Laboratory decreased the muon flux incident on our shield from $\sim 200$ muons per second to $<1$ muon per minute, reducing the rate of neutrons seen from 1 neutron $/ \mathrm{kg} /$ day to 1 neutron $/ \mathrm{kg} /$ year and increasing our sensitivity to WIMP detection by a factor 
of 100 .

\subsubsection{Mean Muon Energy}

A knowledge of the flux and energy spectrum of muons at a deep site is necessary to correctly interpret such backgrounds. As is well known, the muon flux reduces with depth, which is the reason rare particle searches converge underground.

An important measure for quantifying the muon-induced neutron production is the mean muon energy measured at the detector, taking into account as it does both the depth and the geometry of the rock overburden [10][31]. This value may be considered to characterize the muon energy spectrum. With more energetic muons having greater penetration power, the mean muon energy increases with depth. Figure 4.4 shows the variation of mean muon energy with depth as determined by a range of different analytical studies and direct measurements. The production mechanisms that generate muon-induced neutrons underground are listed as follows:

- Negative muon capture by a nucleus

- Direct muon-induced spallation

- Neutron production by hadrons in muon-generated hadronic showers.

- Neutron production by photons in muon-induced electromagnetic showers.

The following sections consider these processes in greater detail and discuss how the contribution of each process varies according to the depth and the energy of the incident muon flux. 


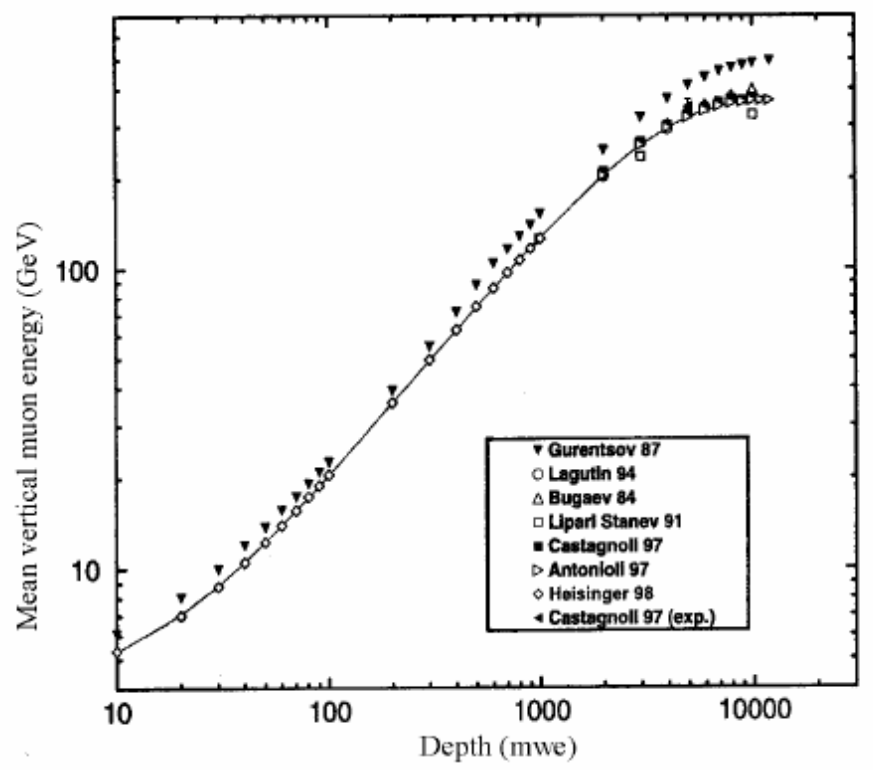

Figure 4.4: Variation of mean vertical muon energy with depth based on calculations[11] [12] and simulations [13][14] (taken from [15]). 


\subsubsection{Muon Capture by Nuclei}

In the first process, slow moving negative muons are captured by nuclei via

$$
\mu^{-}+p \longrightarrow n+\nu_{\mu}
$$

The resulting excited nucleus de-excites by direct emission or evaporation of neutrons. The average number of neutrons produced per muon capture event depends on the material and may vary from 0.7 to $1.7[16]$. Neutrons produced by this process matter only up to depths of $<100$ m.w.e[1]. Since they have energies of a few $\mathrm{MeV}[17][18]$, they are easily moderated by passive hydrocarbon shielding.

Deeper underground, the number of stopping muons is negligible and this process does not contribute much to the overall neutron production. Muon capture thus assumes importance only for the SUF phase of the CDMS experiment.

\subsubsection{Neutrons from Electromagnetic Showers}

Fast muons can produce neutrons though photo-nuclear interactions mediated by real and virtual photons. Processes like bremsstrahlung, pair production and the production of delta rays (knock-on electrons of high-energy produced from Coulomb-scattering interactions of muons with electrons) produce electromagnetic showers. The photons in the showers generate neutrons through $(\gamma, n)$ and $(\gamma, x n)$ reactions. The spectrum of shower-inducing photons falls off as $\mathrm{E}_{\gamma}^{-2}$, where $E_{\gamma}$ is the photon energy for $E_{\gamma} \ll E_{\mu}$, the mean muon energy[19]. The energy spectrum of the neutrons is similar to that of muon capture as neutron production follows the direct emission or evaporation path.

At 17 m.w.e. depth, where the mean muon energy is $\sim 8 \mathrm{GeV}$, as indicated by Figure 4.3, muon-induced neutrons generated in the shield resulted primarily from these two production mechanisms $[6]$. 


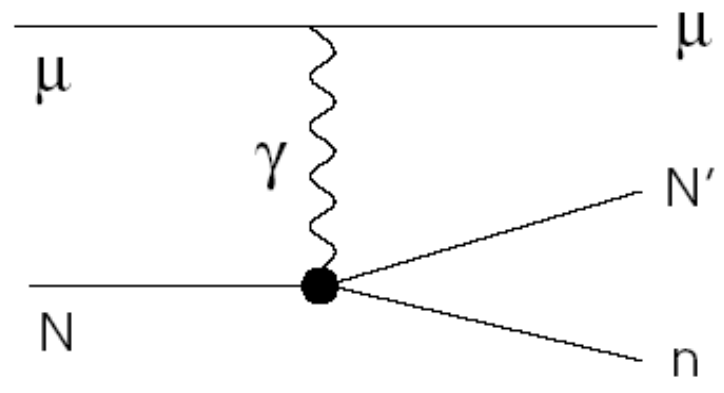

Figure 4.5: Muon spallation

\subsubsection{Muon-nucleus Inelastic Scattering}

When high-energy muons interact with nuclei through a virtual photon, the process is termed as muon-nucleus inelastic scattering. When the inelastic interaction of the primary muon with a nucleus via a virtual photon produces a nuclear disintegration, direct muon-induced spallation results. The Feynman diagram of a typical spallation reaction is shown in Figure 4.5.

The virtual photon of the muon's electromagnetic field interacts with the electric dipole moment of the nucleus, resulting in the absorption of the photon by the nucleus which drives the latter into an excited state. If the absorbed photon has a large enough energy, the nucleus undergoes a transition to another nucleus with the resultant emission of one or more neutrons or other hadrons.

The energy of the initial photon determines the energy of the neutrons produced. For photons of energies $<10-30 \mathrm{MeV}$, a photon is absorbed and the nucleus can decay following the emission of one or more nucleons. The resulting neutrons have energies of a few $\mathrm{MeV}$, corresponding to the nuclear binding energy per nucleon, similar to neutrons from slow muon capture.

As with muon capture, evaporation and direct emission are the principal neutron generating mechanisms. Neutrons in electromagnetic showers are generated 
mainly by low-energy photons.

With increasing photon energies of the order of 10 - $30 \mathrm{MeV}$, a giant dipole resonance due to the nuclear electric dipole moment translates into a large increase in the cross section for photo-nuclear interactions. Nucleons are emitted as before with the decay of the nucleus, with similar energies of the order of $<10 \mathrm{MeV}$. Neutrons produced thus have a spectrum similar to those produced from the evaporative process and can be easily moderated by the use of passive shielding.

Between $30-150 \mathrm{MeV}$, the neutron is emitted with half the energy of the photon, of the order of $15-75 \mathrm{MeV}$, minus its binding energy, for light and medium nuclei[19]. When the energy crosses the pion production threshold (140 $\mathrm{MeV}$ ), the photons interact with individual nucleons in a nucleus to produce highenergy hadrons through:

$$
\begin{aligned}
& \gamma+p \\
& \gamma+n \longrightarrow n+\pi^{+} \\
& \longrightarrow p+\pi^{-}
\end{aligned}
$$

The resulting high-energy pions, neutrons and protons may all interact with other nuclei to produce more neutrons and other particles through hadronic cascades. For pions, this follows the $\pi$ - nuclear interaction:

$$
\pi^{-}+(Z, A) \rightarrow(Z-1, A-1)+n
$$

The energy of the cascade neutrons falls off as $\mathrm{E}^{-1 / 2}$ between $10-50 \mathrm{MeV}$ and more sharply at higher energies.

Equation 4.5 is of particular importance to dark matter searches such as ours as it yields neutrons of several hundred $\mathrm{MeV}$ which are able to travel far enough from the cascade track to punch through the shielding material, giving rise to secondaries in the shield which produce nuclear-recoils in the same energy range as an expected WIMP signal.

At the shallow site, this was our limiting background[20]. 


\subsection{Uncertainties in the Neutron Yield}

Inelastic muon interactions produce neutron backgrounds that can limit sensitivity to WIMP detection. Unfortunately these backgrounds happen to be plagued by uncertainties, specially in estimates of the total neutron yield from muon spallation and the cascade generating secondary processes.

Various underground experiments [21][22][23][24] have used the FLUKA[25] and, in some cases GEANT4[26], particle transport programs to model the neutrons produced by hadronic and electromagnetic cascades initiated by muons and make predictions about the yield, spectrum, multiplicity and angular distributions of these neutrons and estimate the uncertainties. The primary muon spallation process has been studied[27] by allowing a $190 \mathrm{GeV}$ muon beam (corresponding to the mean muon energy at depths $\sim 2000$ m.w.e) to be incident on different materials.

In this section, I will present the current state of knowledge on estimates of neutron yield from such processes based both on simulations and experimental studies. In the following sections, and in subsequent chapters, I will use these results to make similar estimates on the neutron flux seen at the shallow and deep sites of the CDMS experiment.

The number of neutrons produced per muon $\mathrm{N}_{\mu}$ per unit path length in nuclear cascades increases with the mean muon energy $\mathrm{E}_{\mu}$ at a site, allowing the latter to be a reasonable indicator of neutron yield. The dependence has been shown[28][29][30] to be:

$$
N_{\mu} \propto E_{\mu}^{0.75 \pm 0.05} .
$$

The neutron flux density per muon also depends on the mean muon energy as a consequence. As depth, and hence mean muon energy increases, more neutrons are produced for every muon in the nuclear cascades. So, while depth should give rise to a reduction in the muon, and hence the muon-induced neutron flux, this increase 
in the neutron multiplicity cause the neutron flux to decrease at a slower rate than the muon flux with depth.

Attempts to quantify this dependence of the total neutron yield on the mean muon energy at a site have been made both by simulations and actual measurements. Various Monte Carlo simulations have been run to quantify the average number of neutrons generated by muons per unit path length $\left(\mathrm{g} / \mathrm{cm}^{2}\right)$ of a material underground. A FLUKA simulation of the neutron production from mono-energetic muons has estimated [10] the total neutron yield per muon in a liquid scintillator $\mathrm{C}_{10} \mathrm{H}_{22}$ and leads to a parametrization of the form:

$$
N_{\mu}=4.14 \times\left(E_{\mu}^{0.74}\right) \times 10^{-6} \mathrm{n} / \mu / \mathrm{g}_{-} \mathrm{cm}^{-2}
$$

where $\mathrm{E}_{\mu}$ is the muon energy in $\mathrm{GeV}$. This yield results from processes such as muon spallation and neutron production from muon induced nuclear and electromagnetic cascades and is consistent with the original predictions in [31]. The contribution to the yield from hadronic cascades has been shown[31] to increase with depth. This has implications for the nature of the spectrum of neutrons produced from muon interactions as will be shown in Chapter VIII for the case of neutron production in the Soudan environment.

Similar modelling using FLUKA was done using various target materials, including liquid scintillator, by a different experimental group. Their results[22] indicate a power-law dependence of yield on $A$, the atomic weight of the target material:

$$
N_{\mu}=(5.33 \pm 0.17) \times\left(A^{0.76 \pm 0.01}\right) \times 10^{-5} \mathrm{n} / \mu /{\mathrm{g}-\mathrm{cm}^{-2}}^{-}
$$

As the cross-section of the radiative processes of the primary muon varies as $Z^{2} / A$, the contribution to the total neutron yield from electromagnetic showers increases with heavier target materials. This dependence on atomic weight is seen in the 
contribution to the yield from neutron producing processes initiated by low-energy photons in electromagnetic showers.

Where measurements of the absolute muon energy spectra are available, it has been estimated[22] that the neutron production is smaller by $10-15 \%$ than by considering the one from muons, all having the energy equal to the mean energy of the spectrum. This holds good for depths from surface to $3 \mathrm{~km}$.w.e. below and for mean muon energies between $100-300 \mathrm{GeV}$.

\subsection{The Neutron Flux at SUF}

Given the importance of the neutron backgrounds at SUF, I will cover this subject in greater detail in later chapters. In Chapter V, I will study the muoninduced neutron backgrounds produced in the shield as I demonstrate how various event populations in the data served as checks on the GEANT3 simulations of the neutron backgrounds. In Chapter VI, I will use the output of the simulations of the muon-induced neutrons produced in the rock, the so-called "punch-throughs" to inform the analysis of the WIMP candidate data set. This section gives an overview of the various neutron fluxes seen at SUF, assessing their relative contribution in affecting the sensitivity to dark matter detection.

The first stage of the CDMS experiment was located at $17 \pm 1$ m.w.e. depth. This reduced the integrated muon flux to 44.4 muons $/ \mathrm{m}^{2} / \mathrm{s}$ [32], only a factor of 4 below the value of 180 muons $/ \mathrm{m}^{2} / \mathrm{s}$ at sea level.

The 25-cm outer polyethylene shield effectively suppressed the contribution from neutrons arising from natural radioactivity and spontaneous fission from the rock of the cavern and surrounding material. As pointed out earlier, neutrons from such processes in the material within the polyethylene can safely be neglected.

The depth was enough to make the hadronic component of cosmic rays negligible. As regards the contribution from the muon-induced neutrons, I will divide them in two categories: low-energy neutrons ( of energies up to $20-30 \mathrm{MeV}$ ) 
produced from muon capture, and low and medium energy photo-nuclear reactions; and the fast neutrons generated by very high-energy photo-nuclear reactions from hadronic showers initiated by the muons.

\subsubsection{Muon-Induced Neutrons in the Shield}

Muon capture and low-energy photo-nuclear interactions inside the shield produce an important background but one that is rejected by the scintillator muon veto. As explained in Chapter $\mathrm{V}$, only the contribution from the inner lead and the copper cans of the icebox is significant. Based on measured production rates in lead[6] and estimates in copper, the neutron rate has been estimated to be $1 \mathrm{x}$ $10^{4}$ /day from low-energy photo-nuclear interactions and $3.2 \times 10^{4}$ /day from muon capture in the inner lead and copper cans[33]. The spectral distribution follows:

$$
\frac{d N}{d E}=\left\{\begin{array}{c}
0.812 E^{5 / 11} e^{\left(-\frac{E}{1.22}\right)} \text { for } \mathrm{E}<4.5 \mathrm{MeV} \\
0.018 e^{\frac{-E}{9}} \text { for } \mathrm{E}>4.5 \mathrm{MeV}
\end{array}\right\}
$$

This is the "internal" neutron flux. Though vetoed out of the dark matter analysis, the fact that it occurs throughout a data run allows for its use as an important means of calibrating the detector response to a large sample population of nuclearrecoil events over a period of time.

high-energy photonuclear interactions induced by muons in the shield generate very high-energy neutrons with relatively small interaction cross-sections in the detectors. The rate of such neutrons produced in the material within the polyethylene is $\sim 4 / \mathrm{kg} /$ day[6]. This contribution is small in comparison to the more dominant rates $(\sim 100 / \mathrm{kg} /$ day in copper and $243 / \mathrm{kg} /$ day in lead) due to muon capture. high-energy neutrons produced in this manner in the outer lead can punch through the polyethylene and leave signals in the detectors but, again, their contribution is small $(\sim 5 \%)$ compared to the muon capture neutrons. 


\subsubsection{The Limiting Background at Stanford}

Neutrons from muon capture and low-energy photonuclear interactions in the surrounding rock, though unvetoed, are easily suppressed by the outer polyethylene and do not significantly contribute to the final neutron tally.

High-energy photonuclear interactions induced by muons in the rock and the associated hadronic showers produce high-energy neutrons that "punch through" the shield producing secondaries that can mimic the WIMP signal. This background has been documented in [8]. The energy distribution follows:

$$
\frac{d N}{d E}=\left\{\begin{array}{c}
6.05 e^{\left(-\frac{E}{77}\right)} \text { for } \mathrm{E}<200 \mathrm{MeV} \\
e^{\frac{-E}{250}} \text { for } \mathrm{E}>200 \mathrm{MeV}
\end{array}\right\}
$$

being independent of the nature or energy of the projectile $(\pi, \gamma, n, p)$ provided this energy exceeds $2 \mathrm{GeV}[29]$. The rate of neutron production is taken as (4.14 $\pm 0.3)$ neutrons $/ \mathrm{kg} /$ day of $\operatorname{rock}[34]$ which yields a flux of $(2.14 \pm 0.02) \times 10^{-6}$ neutrons $/ \mathrm{cm}^{2} / \mathrm{s}$ incident on the detecting apparatus.

However, there is a factor of 3 uncertainty associated with the rate obtained from literature. The neutron production rate in rock at 17 m.w.e. has been poorly quantified by earlier measurements. Additional uncertainty arises from the fact that a certain fraction of these neutrons $(\sim 40 \%)$ trigger the veto. Charged particles in the hadronic showers associated with the neutron production can also trigger the veto, causing the data analysis to reject such events.

Given these uncertainties, it is difficult to get a measure of the production rate of these neutrons. For Run 21, we made use of the fact that an earlier data run at the same site had arrived at a set of candidate events that was consistent with all events seen being neutrons[20] [35]. The results of the earlier run were used to normalize simulations of the external neutron background to remove the factor of 3 uncertainty associated with the rate. The procedure has been explained in Chapter VI. 


\subsection{The Flux at Soudan}

At Soudan, most of the neutrons seen arise from natural radioactivity and spontaneous fission. The muon flux is reduced from 44.4 muons $/ \mathrm{m}^{2} / \mathrm{s}$ at the Stanford Facility to $(2.21 \pm 0.03) \times 10^{-3} / \mathrm{m}^{2} / \mathrm{s}$ at the deep site[36]. (An alternate measurement puts the muon flux at $\left.1.8 \times 10^{-3} / \mathrm{m}^{2} / \mathrm{s}[7]\right)$. At this depth, muoninduced neutron production is dominated by secondaries arising from hadronic and electromagnetic showers initiated by inelastic muon-nucleus scattering. As stated

earlier, the total neutron yield from such muon induced processes shows a $\mathrm{E}_{\mu}^{0.7}$ dependence on the mean muon energy at a site. At Soudan the mean muon energy has been measured to be $210 \mathrm{GeV}$ [7].

There have been varying measurements of the muon flux at the Soudan site. The Soudan II detector studies estimated the muon flux averaged over all zenith angles as $1.80 \times 10^{-3} / \mathrm{m}^{2} / \mathrm{s}[7]$. An alternate measurement using a muon telescope places this value at $(2.21 \pm 0.03) \times 10^{-3} / \mathrm{m}^{2} / \mathrm{s}[36]$. The $20 \%$ increase in the flux in the second case may have arisen from including hadronic showers in the muon estimates. We fold in the two values of the flux as uncertainties in the measurement and set the muon flux to be $(2.0 \pm 0.2) \times 10^{-3} / \mathrm{m}^{2} / \mathrm{s}$.

\subsubsection{Neutrons from the Shield}

Following the same line of reasoning as for the shallow site, it is clear that the principal contribution to the muon-coincident neutrons produced in the shield comes from the lead in the shielding. Not only does lead have a high neutron yield, but at Soudan, it is located within most of the polyethylene, allowing for just 7.6 $\mathrm{cm}$ of polyethylene interior to the lead to shield the detectors from the lower energy neutrons produced in it. The muon-induced neutron flux has a high-energy (50 - 600 $\mathrm{MeV}$ ) component from hadronic cascades resulting in low interaction cross-sections in the detectors and lower energy $(<50 \mathrm{MeV})$ neutrons produced by muon-nuclear interactions mediated by low-energy photons. 
We make the assumption that the high-energy component follows the spectral distribution given by Equation 4.10 for neutrons generated in hadronic showers from very high-energy photo-nuclear interactions. A FLUKA simulation generating the neutron spectrum from muons incident on lead at a depth of 2450 m.w.e shows a reasonably good agreement[21] with this distribution.

While muon capture may be essentially ignored at this depth due to the paucity of slow moving muons, lower energy neutrons are produced from muonnuclear interactions mediated by lower energy real or virtual photons in electromagnetic or nuclear cascades. The spectrum of lower energy shower neutrons can be considered to eventually approach the evaporative form given by Equation 4.9. A FLUKA simulation[21] starting from the primary muon tracks produces a neutron spectrum that corroborates such an assumption.

For the neutron production in lead at the Soudan depths, we draw from both experimental measurements and simulations [37][38][54][55] to make estimates of the average yield . For a mean muon energy of $110 \mathrm{GeV}$, the average yield per muon per unit path length in lead has been found to be $1.81 \times 10^{-3}$ neutrons/ muon $/\left(\mathrm{g} / \mathrm{cm}^{2}\right)$ in lead. By folding in the dependence on mean muon energy as specified in Equation 4.6, and using $\mathrm{E}_{\mu}=210 \mathrm{GeV}$ for Soudan, we get a production rate of $2.9 \times 10^{-3}$ neutrons $/ \mathrm{muon} /\left(\mathrm{g} / \mathrm{cm}^{2}\right)$ of neutrons in lead. A similar computation[24] of the neutron yield at a different depth based on this dependency on mean muon energy shows agreement with a simulation computing the neutron yield at that depth. Specifics of the calculations of the rates and spectra as well as the simulations of the neutron background are in Chapter VIII. This follows the presentation in Chapter VII of the data taken at Soudan.

\subsubsection{Contribution from the Rock}

The unvetoed component of the neutron flux consists of neutrons generated by muon interactions in the rock and those produced by natural radioactivity and spontaneous fission in the rock. The $25 \mathrm{~cm}$ polyethylene shield effectively suppresses 
all but the very high-energy component arising from hadron cascades initiated by muon-nuclear interactions. This is similar to the SUF case, except with a vastly reduced flux and so we use the same spectrum as Equation 4.9 to model these neutrons.

For the higher energies $(>200 \mathrm{MeV})$, the spectral distribution agrees well with the $e^{(-E / 230 M e V)}$ distribution quoted in [7]. Chapter VIII goes into more details on the specifics of the simulation.

The external neutron rate can be determined given that the average number of neutrons produced by a muon per unit path length $\left(1 \mathrm{~g} / \mathrm{cm}^{2}\right)$ is $(3.3 \pm 1.0) \mathrm{x}$ $10^{-4}[7]$ and the integrated muon flux at the site is taken as $(2.0 \pm 0.2) \times 10^{-3} / \mathrm{m}^{2} / \mathrm{s}$. The neutron production rate from cosmic ray induced interactions in the rock has been determined to be $(7.2 \pm 2.2) \times 10^{-8}$ neutrons $/ \mathrm{kg} / \mathrm{s}$.

An alternate value determined from a theoretical calculation of the average neutron yield from various muon induced processes per muon per unit path length in a material at different depths[41] puts the yield at $4.1 \times 10^{-4}$ neutrons per muon per unit path length, which is within the uncertainties specified in [7]. By using the production yield in lead at a depth of $270 \mathrm{GeV}$ and folding in the $\mathrm{E}_{\mu}^{0.75}$ dependency for the corresponding value at the mean energy at Soudan, we get $2.95 \times 10^{-4}$ neutrons $/ \mu /\left(\mathrm{g} / \mathrm{cm}^{2}\right)$, again within the uncertainties given.

A third estimate, applying the Soudan muon energies to the total neutron yield produced from an incident muon flux based on FLUKA simulations [10] arrives at a number that also lies within the uncertainties specified above. Details may be found in Chapter VIII, along with specifics on rates, spectra and the simulations modelling the unvetoed background follow in the discussion of the Soudan phase of the experiment. 


\subsubsection{Limiting Sensitivity}

A rare signal is best heard when the background noise is muted, or at least, considerably reduced. It is this principle that persuades WIMP-searches to strive for very low background conditions. As explained in Chapter II, the sensitivity of an experiment is directly proportional to the product of detecting mass $\mathrm{M}$ and exposure time $\mathrm{T}$ in the absence of backgrounds.

With backgrounds present, the rate of progress reduces to $\sqrt{M T}$ owing to statistical fluctuations in background subtraction. Ultimately, the background subtraction may be limited by the systematics involved in calibrating the detector response to the background. Beyond this point, increasing the exposure does not realize additional improvements in sensitivity.

Un-vetoed neutrons backgrounds, like the high-energy component produced by muon-induced hadronic showers in the rock, are the most worrisome for dark matter searches like ours where the detectors are unable to distinguish between a neutron and a possible WIMP signal. Various methods have been considered to tackle such backgrounds - go even deeper underground, add more passive shielding, introduce an active neutron veto into the shielding arrangement, or devise a means of tagging the charged particles that accompany neutron production in the rock to be able to veto such events.

In the final chapter of the thesis, I will summarize some of these methods proposed to reduce the muon-induced neutron flux in a bid to achieve the ideal very low-background environment conducive for a rare particle search. 


\section{References}

[1] M. Aglietta et al, Il Nuovo CimentoC, 12, 467 (1989).

[2] D. Lal and B. Peters, Handbuchder Physik, 46/2, 551 (1967).

[3] G. Heusser, Ann. Rev. Nucl. Part. Sci. 45, 543 (1995).

[4] T. Florkowski et al, Nuclear Geophysics, 2, 1 (1988).

[5] E. Segre, Nuclei and Particles, Second Edition W A Benjamin Inc. (1977).

[6] A. da Silva et al, Nucl. Instrum. E3 Meth. in Phys. Res. A, 354, 553 (1995).

[7] K. Ruddick, MINOS Internal Note, NuMI-L-210 (1996).

[8] T. A. Perera, Ph.D. Thesis, Case Western Reserve University (2002), unpublished.

[9] G. Chardin and G. Gerbier, Proc. of the IV Internat. Workshop on Identification of Dark Matter, 470 (2002).

[10] Y-F. Wang et al, Phys. Rev. D, 64, 013012 (2001).

[11] Heisinger, B., Ph.D. Thesis, Technical University of Munich (1998), unpublished.

[12] Gurentsov, V.I. et al., Sov. J. Nucl. Phys., 23, 527 (1976).

[13] Castagnoli, C. et al., Astropart. Phys., 6, 187 (1997). 
[14] Antonioli, P. et al., Astropart. Phys., 7, 357 (1997).

[15] H. Wulandari, Ph.D. Thesis, Technical University of Munich (2003).

[16] B. Macdonald et al. Phys. Rev.B, 139, 5 (1965).

[17] P. Singer, Springer Tracts in Modern Physics, 71, 39 (1974).

[18] W. U. Schröder, Ph.D. Thesis, Technische Hochschule Darmstadt (1971).

[19] J. C. Barton, Proceedings of the 19th International Cosmic Ray Conference, 8, 98 (1985).

[20] R. Abusaidi et al, Phys. Rev. Lett., 84, 5699 (2000).

[21] J. M. Carmona et al, hep-ex/0403009 (2004).

[22] V. A. Kudryavtsev et al, Nucl. Instrum. \& Meth. in Phys. Res. A, 505, 688 (2003).

[23] M. J. Carson et al, hep-ex/0404042 (2004).

[24] H. Wulandari et al, hep-ex/0401032 (2004).

[25] A. Fasso et al, Proc. of the $4^{\text {th }}$ Intl. Conf. on Calorimetry in high-energy Physics, 493 (1993).

[26] S. Agostinelli et al, Nucl. Instrum. \& Meth. in Phys. Res. A, 506, 250 (2003).

[27] V. Chazal et al, Nucl. Instrum. \& Meth. in Phys. Res. A, 490, 334 (2002).

[28] A. S. Mal'gin et al, JETP Lett., 36, 376 (1983).

[29] F. F. Khalchukov et al, Il Nuovo Cimento C, 6, 320 (1983).

[30] O. G. Ryajskaya and G. T. Zatsepin, Proc. of the IX Internat. Conf. on Cosmic Rays, London, 3, 987 (1966).

[31] L. B. Bezrukov et al., Sov. J. Nucl. Phys., 17, 51 (1973). 
[32] A. da Silva, Ph. D. Thesis, The University of British Columbia, (1996).

[33] S. R. Golwala, Ph.D. Thesis, University of California at Berkeley (2000), unpublished.

[34] M. Chen et al, Nucl. Instrum. Es Meth. in Phys. Res. A, 336, 232 (1993).

[35] D. Abrams et al, Phys. Rev. D, 66, 122003 (2002).

[36] R. Nelson, B.S. Dissertation, University of California at Santa Barbara (2003), unpublished.

[37] G. V. Gorshkov et al., Sov. J. Nucl. Phys., 18, 57 (1974).

[38] G. V. Gorshkov et al., Sov. J. Nucl. Phys., 7, 470 (1968).

[39] G. V. Gorshkov et al., Sov. J. Nucl. Phys., 12, 187 (1971).

[40] G. V. Gorshkov et al., Sov. J. Nucl. Phys., 13, 450 (1971).

[41] F. F. Khalchukov et al, Il Nuovo Cimento C, 18, 517 (1995). 


\section{Chapter 5}

\section{Simulating Neutron Backgrounds at a Shallow Site}

A neutron interacting with a ZIP detector leaves a signature akin to that of a hypothetical WIMP making it necessary for us to adopt alternate means to discriminate against it.

The necessity of achieving very low-background conditions for a rare particle search was described in Chapter IV. While our detectors have demonstrated over $99.9 \%$ rejection capabilities against electromagnetic backgrounds such as gammas and betas, estimating and subtracting the neutron contribution is done using ratios of single-scatter events in the germanium detectors (possible WIMPs) to multiple-scatter events in all detectors and single-scatters in the silicon detectors (likely neutrons).

This chapter focuses on how such simulations of the neutron backgrounds are undertaken. The salient features of the GEANT particle transport code used to perform such simulations is covered, including the advantages, and limitations, of this approach.

The rest of the chapter describes the tasks that need to be undertaken before the output of the simulations is allowed to inform the data analysis and 
provide the inputs necessary for background-subtraction techniques to be applied to the WIMP-candidate data set. These tasks include comparing data and simulations for various calibration runs both to study the detector response to a large number of nuclear-recoil events and, more importantly, confirm that the modelling is faithful to the conditions of the run.

While simulations were undertaken for both the shallow and deep site phases of the experiment, this chapter essentially focuses on Run 21, given the importance of the neutron simulations in the context of the run. The following chapter will describe how the simulations informed the data analysis for Run 21 . Chapter VII and VIII will look at the simulations undertaken for the data run at Soudan.

\subsection{Modelling the Neutrons}

Computer models of various backgrounds seen by a detecting apparatus need to be faithful to the manner in which an incident flux propagates through an experimental set-up. The modelling should accurately reproduce the intensity of the incident flux, the propagating mechanisms within the different media that constitute the shield, the particles produced and the interaction cross-sections that characterize such mechanisms.

\subsubsection{The GEANT Particle Transport Code}

Particle transport codes such as GEANT[1] track the passage of elementary particles through an experimental set-up and simulate energy deposits in them. GEANT began life as a tool for high-energy physicists but has today found applications in medicine, astrophysics, space science and low- temperature rare-particle searches such as our experiment.

A GEANT user encodes the experimental configuration using a structure of 
geometrical volumes whose shape, material and tracking-medium parameters may be defined, and then simulates the transport of particles drawing on an extensive library describing the nature of elementary particles and the physical processes detailing their interactions with matter[1].

The simulations covered in this thesis use the GEANT 3.21 particle transport code. The neutron simulations described hereafter draw on the GHEISHA[2], MICAP[3] and FLUKA[4] packages which simulate hadronic interactions. The importance of FLUKA in simulating the muon-induced neutron background seen in dark matter searches was mentioned in the previous chapter.

In the GEANT code, the FLUKA routines deal mainly with hadronic elastic and inelastic interactions. For the propagation of neutrons with energies greater than $20 \mathrm{MeV}$, the GEANT-FLUKA package is used while GEANT-MICAP is employed for the lower-energy component[5]. For the neutrons generated by muoninduced interactions in the rock ("externals") and those produced in the shield ("internals"), the incident flux has been estimated from literature based on previously measured production rates. The production of the neutrons from the muon-induced processes is not simulated and so correlations from a single parent muon, expected to be a small effect at the SUF energies, are not simulated.

To run a simulation, we first code in the geometry of the detector and shielding arrangement. We must also specify the nature of the incident particles as well as their initial positions and three momenta. In GEANT, the units of energy and momenta are $\mathrm{GeV}$ and $\mathrm{GeV} / \mathrm{c}$ while position co-ordinates are given in $\mathrm{cm}$.

Simulating a process involves

- evaluating the probability that the process may occur by sampling the total cross-section;

- generating the final state after the interaction by sampling the differential cross-section;

- determining the energy loss due to radiation and other physical parameters. 
When new particles are produced due to the interactions, their information is stored, allowing for their tracking once the tracking of the original particle is done. The generation of the incident particle is termed as a 'throw'. Throws result in scatters and hits which is how we refer to individual interactions of particles during the propagation.

Our specific area of interest, especially in studying the effect of internal multiple-scatters or in estimating the efficiency of the fiducial-volume to WIMPs, is the hits occurring within the detector material.

An event includes all processes, including the production and interactions of secondaries, that occur between successive throws of a primary particle. An "ntuple"[6] saves information about all events that cause energy to be deposited in the detectors. This includes information about the type of incident particle and its energy, as well as the energy deposited in the detectors by neutrons and other particles for that event. The mechanism of the simulation has been well documented in $[5]$.

\subsubsection{Coding in the Geometry}

The first task in any simulation is to model the experimental set-up. For the Run 21 simulations, the modelling involved coding in the geometry of the detectors and the shield. As explained in Chapter III, Run 21 took place at a shallow site where the relatively high muon flux ensured backgrounds of the order of 1 event $/ \mathrm{kg} /$ day. Reflecting the needs of the site, the shield used a lead-polylead-poly sandwich to shield the detectors against the different backgrounds seen.

While the simulations were mostly true to real conditions, in some cases, the coding was simplified without compromising the results. For example, a cylindrical volume of copper with the same total volume was used instead of modelling the sidecoaxes, SQUET cards and other pieces of cold hardware that make up the detector assembly. This is a reasonable approximation given that the neutron backgrounds we deal with are not localized to the detectors. 


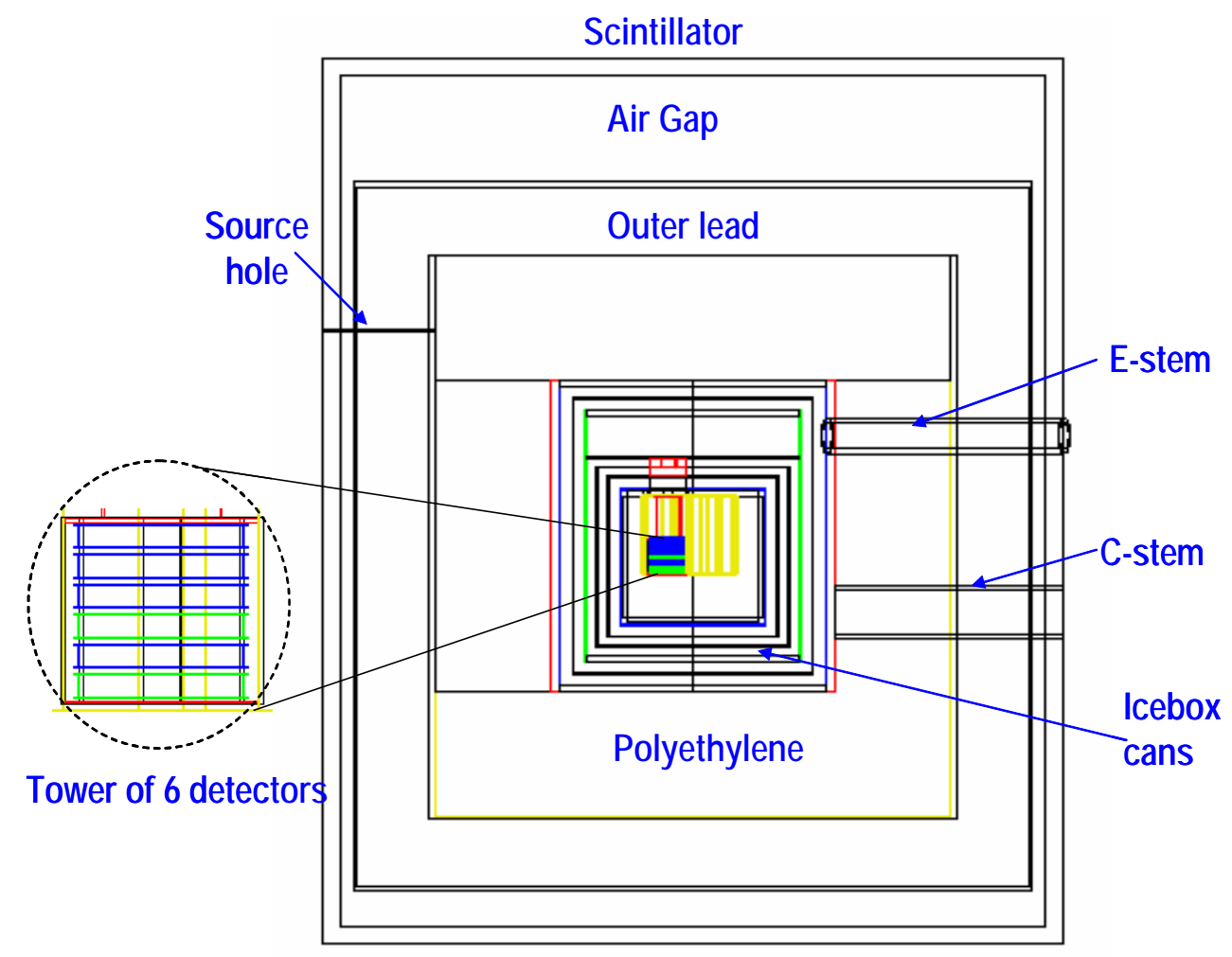

Figure 5.1: Representation of the geometry of the detecting assembly as used in the simulations of the Run 21 neutron background. Shown are the different layers of the shield and icebox with a zoomed in view of the tower with its six detectors.

Besides, since neutrons tend to be a diffusive background, it is more important for the geometry to reflect the correct total mass of the structural components than be true to the specific line-of-sight thickness, as is the case with simulations involving gamma and beta particles. Representations of the geometry of the shield and detectors are shown in Figures 5.1 and 5.2. 


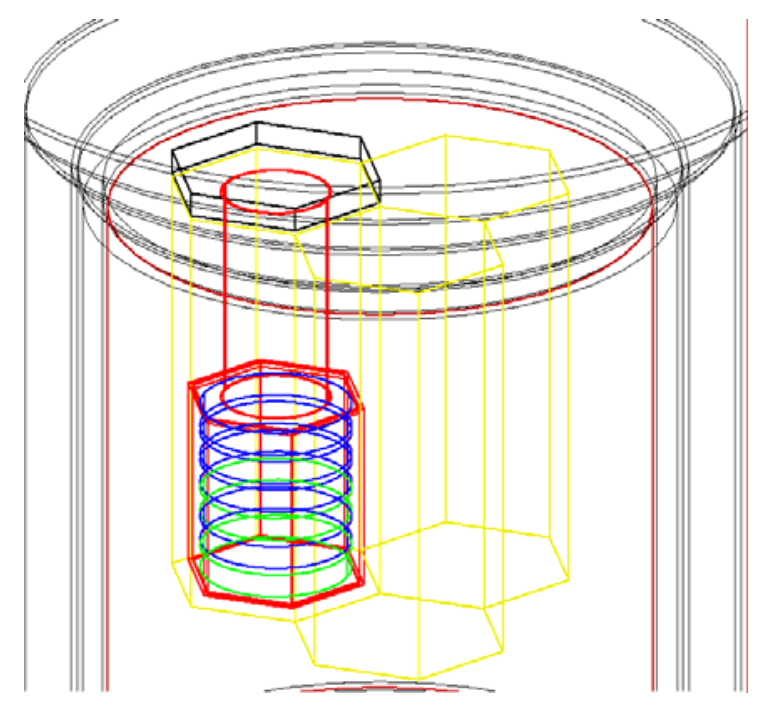

Figure 5.2: A closeup of the tower with the four germanium( green color) and two silicon (blue) detectors. Holes are cut into the inner poly to allow for the placement of the tower assembly.

\subsection{The Neutron Calibrations}

As explained in Chapter III, part of a dark matter run involves conducting periodic calibrations with standard sources to monitor detector response. Such runs are used to set a calibration scale of the charge and phonon channels and identify the signal and background regions. The neutron calibration runs help define the signal region where potential WIMP candidates may be detected. They also allow us to monitor the stability of the nuclear-recoil band to achieve the high level of background discrimination necessary for a WIMP-search.

A ${ }^{252} \mathrm{Cf}$ source with a nominal activity of $0.05 \mathrm{mCi}$ on $23^{\text {rd }}$ July, 1991 was used for the neutron calibrations. ${ }^{252} \mathrm{Cf}$ is a neutron emitter through spontaneous fission. The decay branching ratio is $3.092 \%$ for fission with an average of (3.75 $\pm 0.01)$ neutrons per fission[7]. The energy spectrum shows a peak at around $1 \mathrm{MeV}$ with few neutrons emitted with energies greater than $8 \mathrm{MeV}[8]$. 
The specified activity indicated a neutron production rate of $2.17 \times 10^{5}$ neutrons/second on the date stamped. An alternate measurement [9] comparing the activity of the source with calibrated Plutonium-Beryllium, Plutonium-Boron, Plutonium-Fluorine and Plutonium-Lithium sources suggests that the nominal activity of the source may be $20 \%$ higher than specified.

The calibration runs were performed with the source placed in what were designated as the "side" or the "top" positions (see Figure 5.3). In both cases, the outer polyethylene above the top of the icebox was removed. Polyethylene above the bottom of the icebox on the side facing the source was also removed for the calibration in the side position. This is done to ensure an event rate of $3-4 \mathrm{~Hz}$ on the detectors for the $8-12$ hour period of a typical calibration run.

Neutrons emitted by the ${ }^{252} \mathrm{Cf}$ source also lead to activation of the ${ }^{70} \mathrm{Ge}$ isotope in the germanium detectors. ${ }^{70} \mathrm{Ge}$ absorbs a neutron to form ${ }^{71} \mathrm{Ge}$. This isotope of germanium has a half-life of 11 days and decays to ${ }^{71} \mathrm{Ga}$ emitting a characteristic $10.4 \mathrm{keV}$ photon[10]. The duration of the runs needs to be carefully chosen to balance acquiring enough statistics to define well the signal region while minimizing such activation.

The runs proceeded at two ionization voltages $3 \mathrm{~V}$ and $6 \mathrm{~V}$. For the simulations, the geometry configuration of the data was replicated and the rates and spectra compared for data vs. the modelled output. The next few sections give particulars of representative calibration runs and the simulations that modelled them.

\subsection{Simulating the Calibrations}

The ${ }^{252}$ Cf calibrations were simulated using the GEANT 3 particle transport code assuming a source with the above-mentioned activity and a decay rate based 


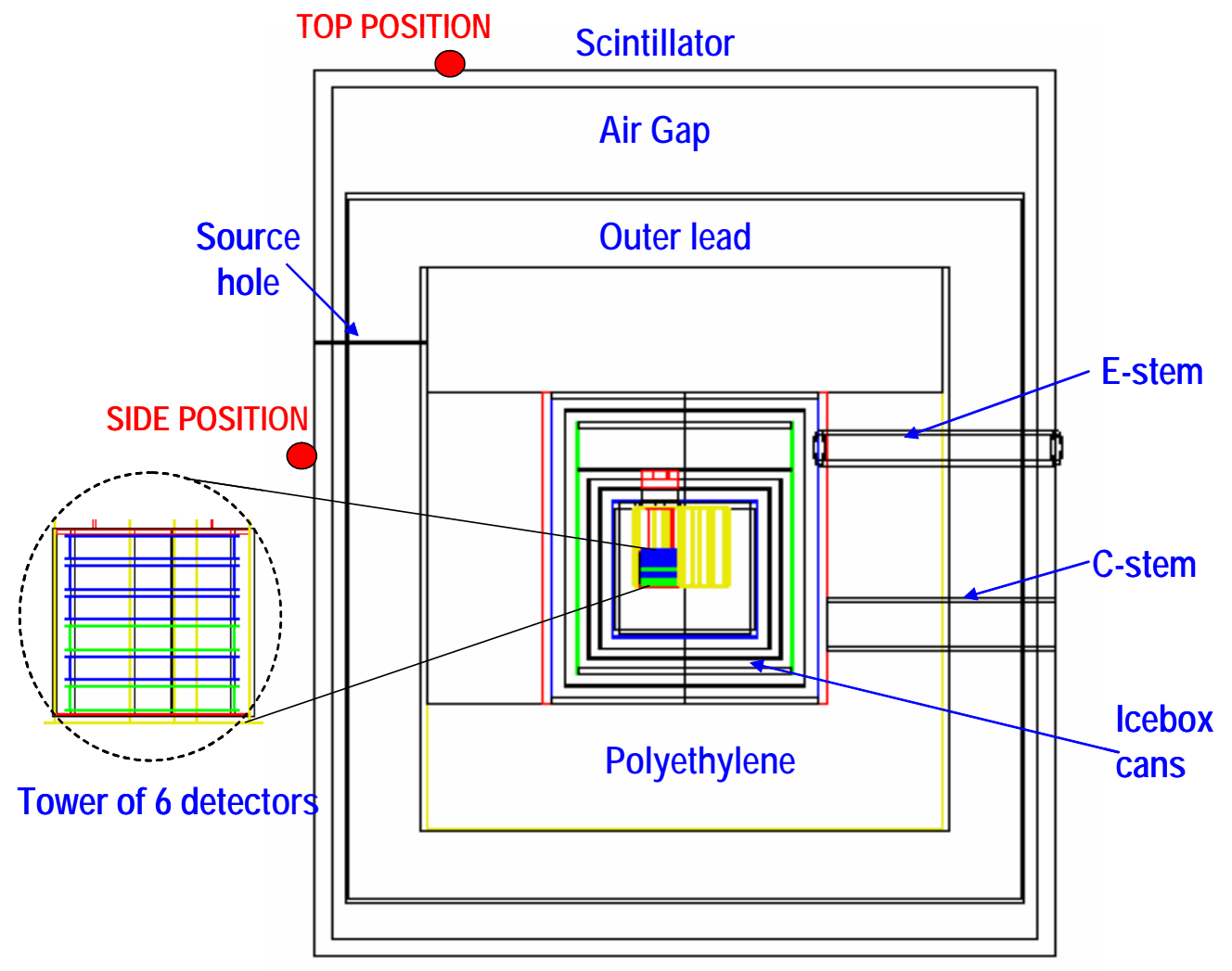

Figure 5.3: Source positions for the ${ }^{252}$ Cf calibration run 
on a half-life of 2.645 years[11]. The neutron production spectrum is given by the Maxwellian distribution[12]:

$$
\frac{d N}{d E}=\sqrt{E} e^{-E / 1.3}
$$

Only the primary neutron emitted by the source and its secondaries are propagated in the simulation. The simulation does not accurately model the case of more than one neutron arising from the same fission event producing recoil energies in the detectors. This may lead to an underestimate in the number of multiple-scatter events seen in a simulation run. The low interaction probability of neutrons ensures that this is not a considerable effect. In one of the simulations, of the $\sim 8.2 \times 10^{8}$ neutrons thrown, only 130286 produced recoil events in the detectors leading to a ratio of 6400 throws for every detector hit.

The case of a photon and a neutron from the same decay producing recoil events in the detectors is also not considered as photons emitted by the source are easily absorbed by the lead in the shield.

The simulations inform the operation of the experiment and the analysis of the data in various ways. Some of them include:

- Testing different source geometries to arrive at an optimal position of the ${ }^{252} \mathrm{Cf}$ source to increase the fraction of direct hits on the detectors and the ratio of neutron to gamma events. Towards this end, short simulation runs testing modifications to the shield such as adding or removing an extra layer of polyethylene and moving the source inside the veto were carried out prior to the end-of-the-run calibrations.

- Testing various shield configurations to check the effectiveness of particular layers in rejecting backgrounds. These kind of tests were done prior to the commissioning of the next stage of the experiment at Soudan. 
- Neutrons from the source multiple-scatter in the shield and eventually fall to thermal energies, releasing a $2.2 \mathrm{MeV}$ photon. This produces a gamma rate during the calibration run which can be quantified using simulations. The computer modelling helps quantify other backgrounds which can impact the event rate on the detectors.

- Calibration runs are utilized to define data cuts for selecting a particular event populations. Simulations of the calibration data provide a large sample of events on which to estimate, and test, the efficiency of the data cuts applied.

\subsection{V Neutron Calibrations}

Three neutron calibration runs were taken at an ionization voltage of $3 \mathrm{~V}$ over the duration of Run 21. The first one was discarded as it coincided with cryogenic problems. The other two runs were taken with the source in different positions, informed by the simulations as to optimal event rate on the detectors.

\subsubsection{First analysis using the flat rise time cut}

The ${ }^{252} \mathrm{Cf}$ source was placed to the side of the shield with the outer polyethylene on that side removed within line of sight of the detectors. The simulations recorded the nuclear and electron-recoil energies for each event that caused energy to be deposited in the detectors. Each detector is partitioned into an inner (85\% of the volume) and an outer region defined by the two charge electrodes that make up part of the sensor. As was explained in Chapter III, the data analysis accepts only the events recorded in the inner charge electrode. A subsequent study more accurately quantified the energy partition based on the fiducial-volume of a detector.

Before comparing with data, it is necessary to fold in the efficiencies of the various cuts applied to the actual output from the detectors. This was done as follows: 
- For each nuclear-recoil hit, the corresponding values of the ionization energies were computed from the centroids of the $3 \mathrm{~V}$ nuclear-recoil bands as formulated for the data. Noise was added to the ionization energies based on the base line noise resolution of $0.8-1.1 \mathrm{keV}$ FWHM for the ionization channels of the detectors. The noise in the outer electrode was taken as $80 \%$ that of the inner sensor, as evidenced from noise studies of the two charge channels in the data analysis.

- Charge and recoil threshold cuts were set as in the case of the data.

- The fiducial-volume was defined away from the edges of the crystal to ensure uniform ionization response.

- The efficiency of an event falling in the signal region was folded in (95\%) for the simulated output reflecting the $2 \sigma$ acceptance of the data cut for the nuclear-recoil band.

- In cases where a veto cut was applied, an $80 \%$ efficiency was folded in corresponding to the efficiency of the data cut. In the neutron calibration, the veto cut is not applied since almost half the events producing recoils in the detectors have interactions in the veto with about $10 \%$ leading to energies of $>2 \mathrm{MeV}$ being deposited in the veto. Had this cut been applied to the WIMP-search data, these events would have been vetoed out of the analysis, reducing the statistics on the calibration.

- A cut was imposed to ensure that events producing simultaneous electronrecoil energies in the detectors were excluded.

The neutron calibration data is shown by detectors in Figure 5.4. For each calibration, the source rate of the ${ }^{252} \mathrm{Cf}$ was calculated based on the stamped value of the source and subsequent activity till the date of the run. Figure 5.5 shows the comparison of data and simulations for all events and for events that have multiple-scatters. A multiple-scatter event requires two or more detectors to pass 
the fiducial-volume and nuclear-recoil band cuts. When the rise time cut was applied as in the case of the veto coincident low background data, the multiplescatter cut was modified as explained in Section 5.5. For the purpose of comparing data and simulations of the neutron calibration, only events with low-yield and lying in the signal region which pass the fiducial-volume cut for the data have been selected. Figure 5.6 shows a similar comparison for events that have singlescatters. A single-scatter event was defined as one occurring in the fiducial-volume and having low yield in the detector in question while all other detectors have less than $2 \mathrm{keV}$ energy deposited in them. The good agreement between data and simulation confirms the computer modelling of the detector response. To compare data and simulations, events are co-added in the four germanium and two silicon detectors as this is how the detector response is tabulated in the WIMP-search data. This helps us make a material wise comparison of the detection rates of neutrons as well as allow for comparison for similar analyses of the WIMP-search and the muon-coincident neutron population. Figure 5.7 and Figure 5.8 show how data compares with the modelled output for the co-added case for both singles and multiples in the detectors. The event rates for single and multiple-scatter events in all six detectors are given in Table 5.1.
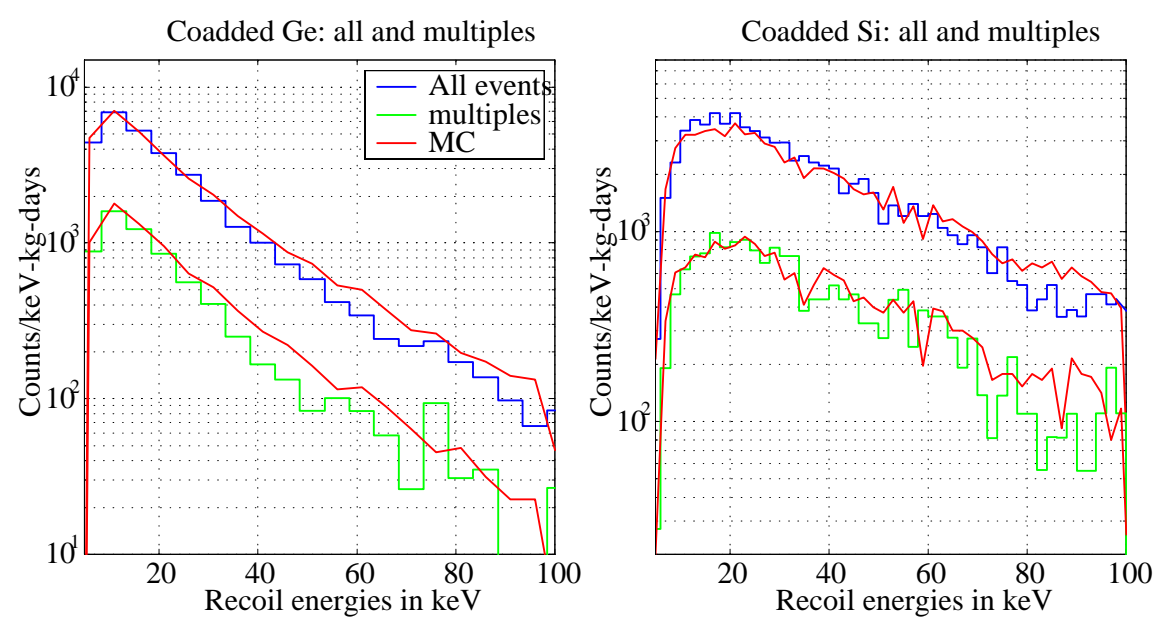

Co-added Ge and Si detectors: All and multiple events 

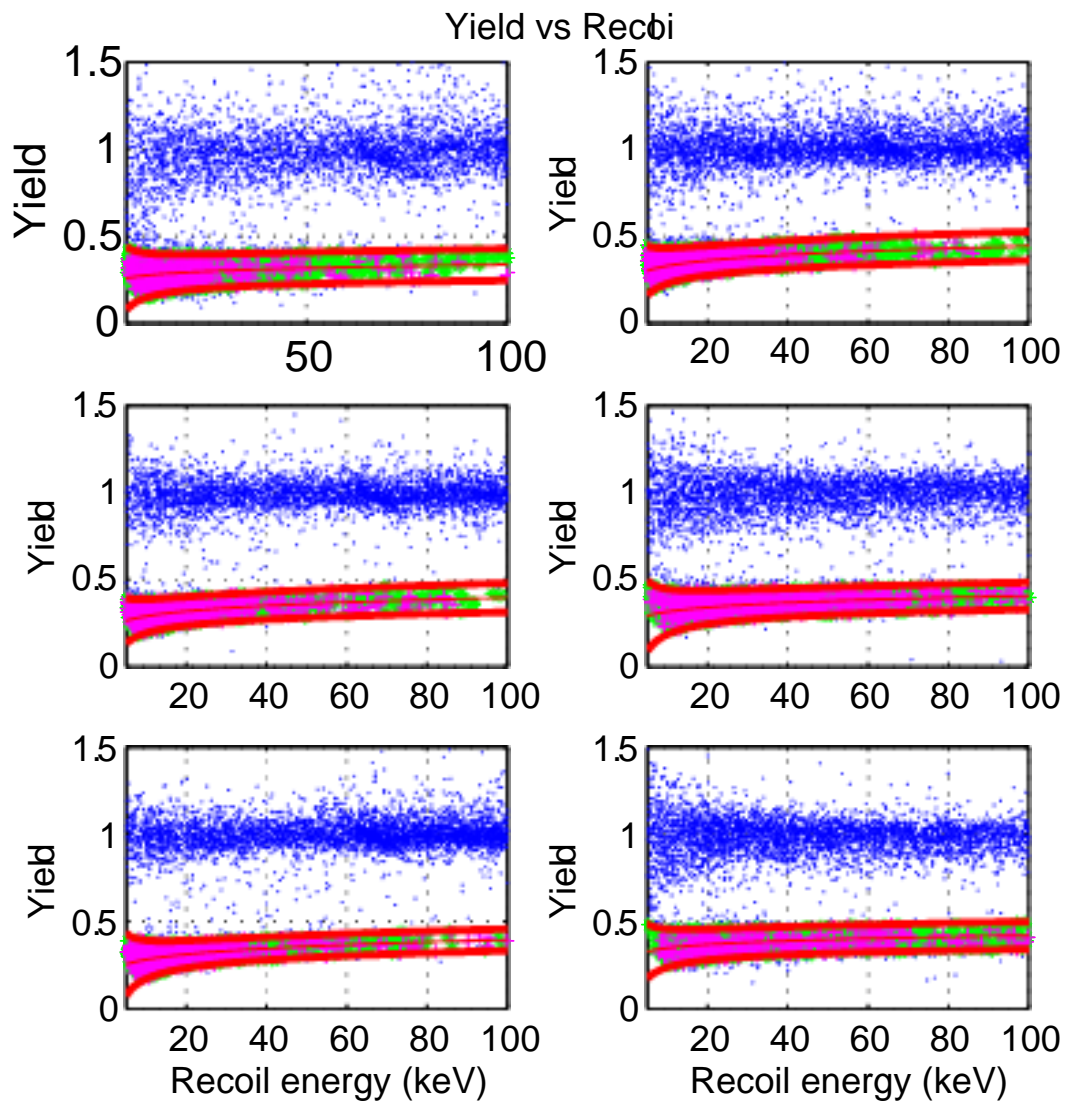

Figure 5.4: Yield vs. Recoil for the 3V Neutron Calibration with the source on side. Detectors Z1 through Z6 are shown from top left to bottom right. Z4 and Z6 are silicon detectors while the other four are germanium detectors. The top band is the background region while the lower band, bounded by red lines defines the signal region. The magenta crosses represent single scatter events in the signal region. 

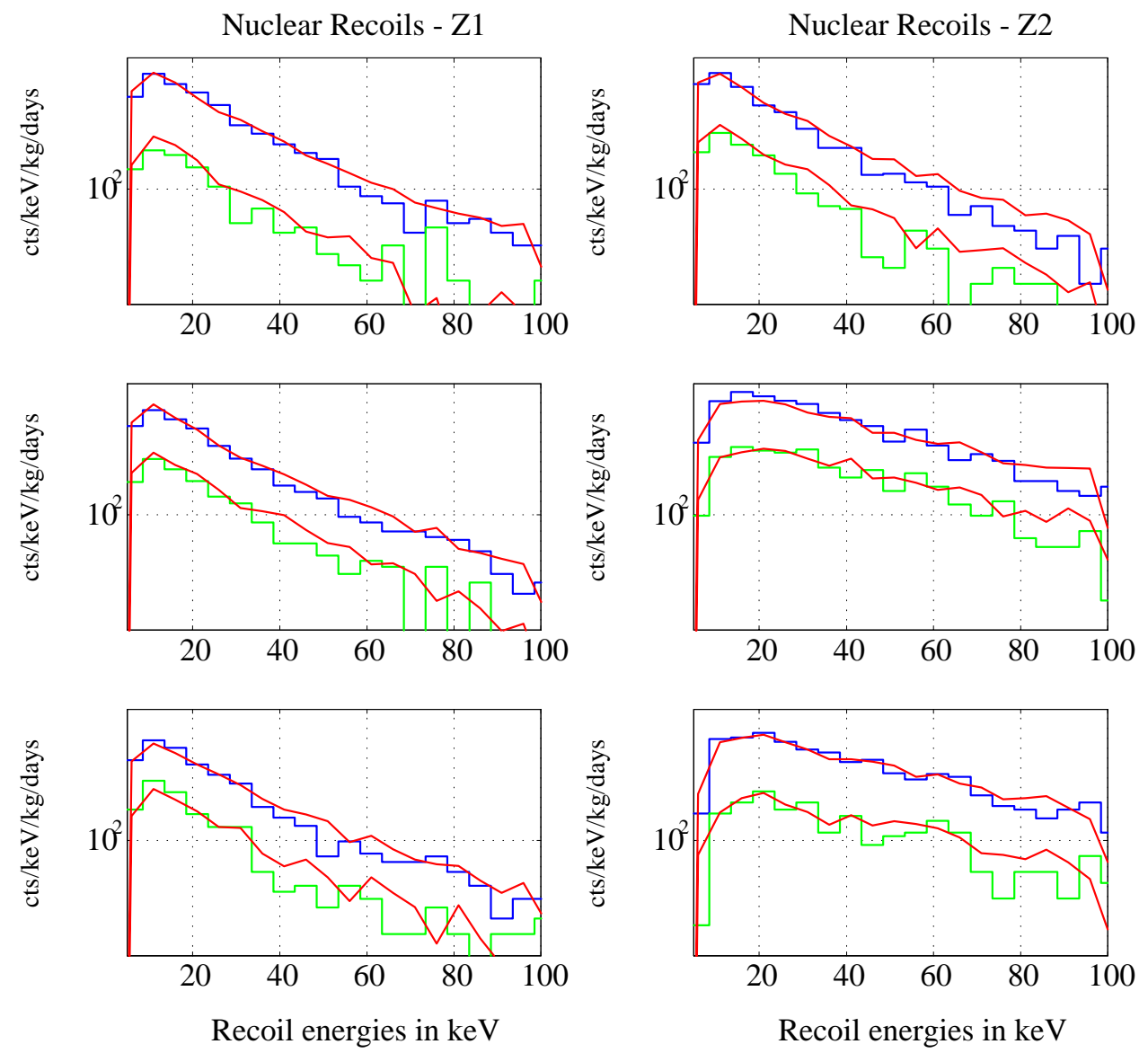

Figure 5.5: Comparison for data and simulation for all events (upper curves) and multiple scatter events (lower curves) for the 3V neutron calibration. The plots show the six detectors from top left to bottom right. The histograms show the data and simulations comparisons for all and single scatter events. 

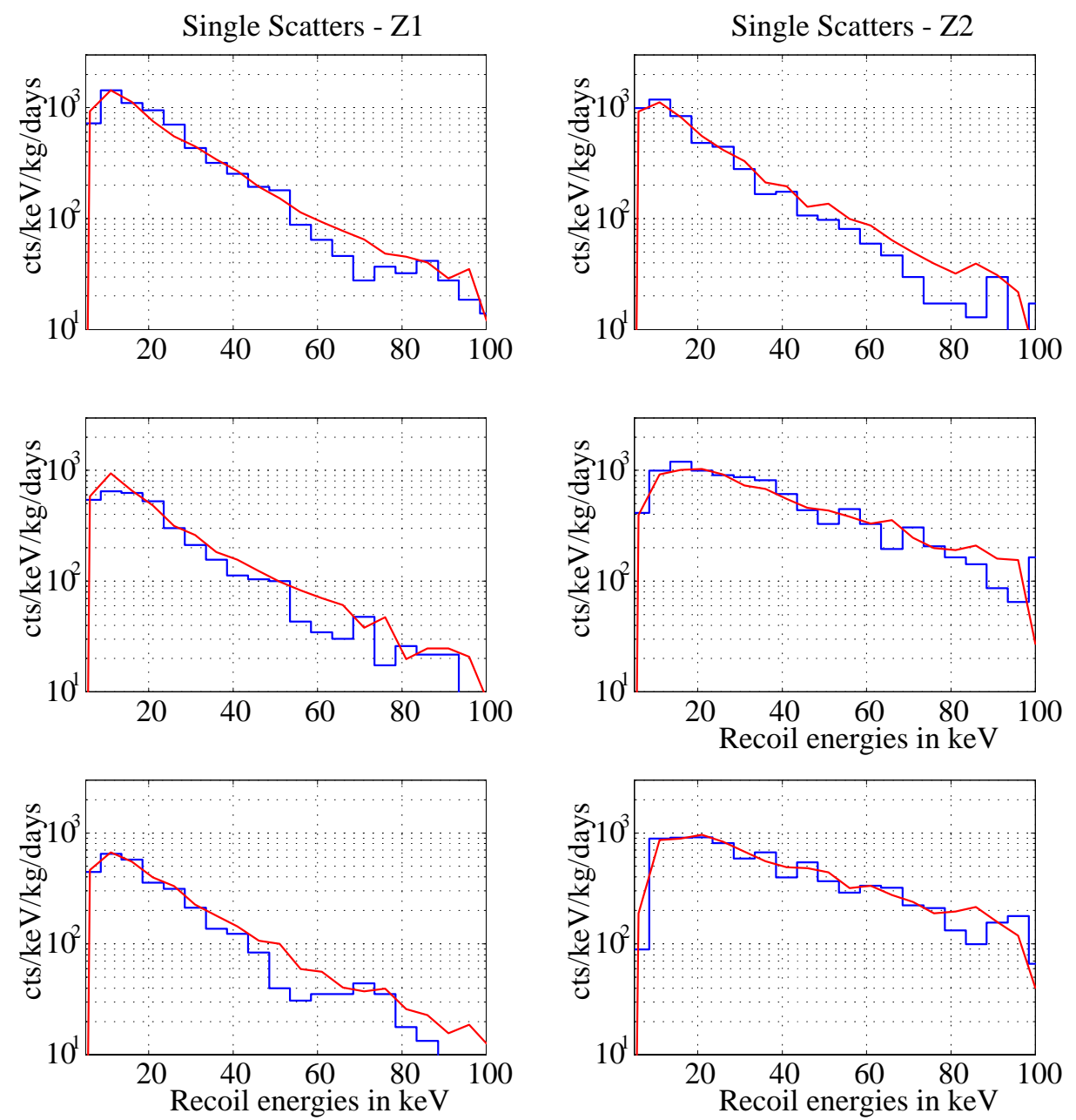

Figure 5.6: Comparison of data histograms and simulations for single scatter events in the $3 \mathrm{~V}$ neutron calibration data. Detectors Z1 to Z6 are shown top left to bottom right. Z4 and Z6 are silicon detectors while the other four are germanium detectors. 


\begin{tabular}{|c|c|c|}
\hline & Data & MC \\
\hline Singles rate in evts $/ \mathrm{kg} /$ day & $(2.63 \pm 0.03) \times 10^{4}$ & $(2.80 \pm 0.02) \times 10^{4}$ \\
\hline Ge singles rate evts/kg/day & $(2.27 \pm 0.03) \pm \times 10^{4}$ & $(2.47 \pm 0.02) \times 10^{4}$ \\
\hline Si singles rate evts/kg/day & $(4.42 \pm 0.01) \times 10^{4}$ & $(4.44 \pm 0.05) \times 10^{4}$ \\
\hline Si singles $/$ Ge singles & 0.389 & 0.359 \\
\hline
\end{tabular}

Table 5.1: Event rates for single scatter events in the four germanium and two silicon detectors for the Run21 3V neutron calibration. The efficiencies of the standard 3V cuts have been folded into the Monte Carlo simulations.
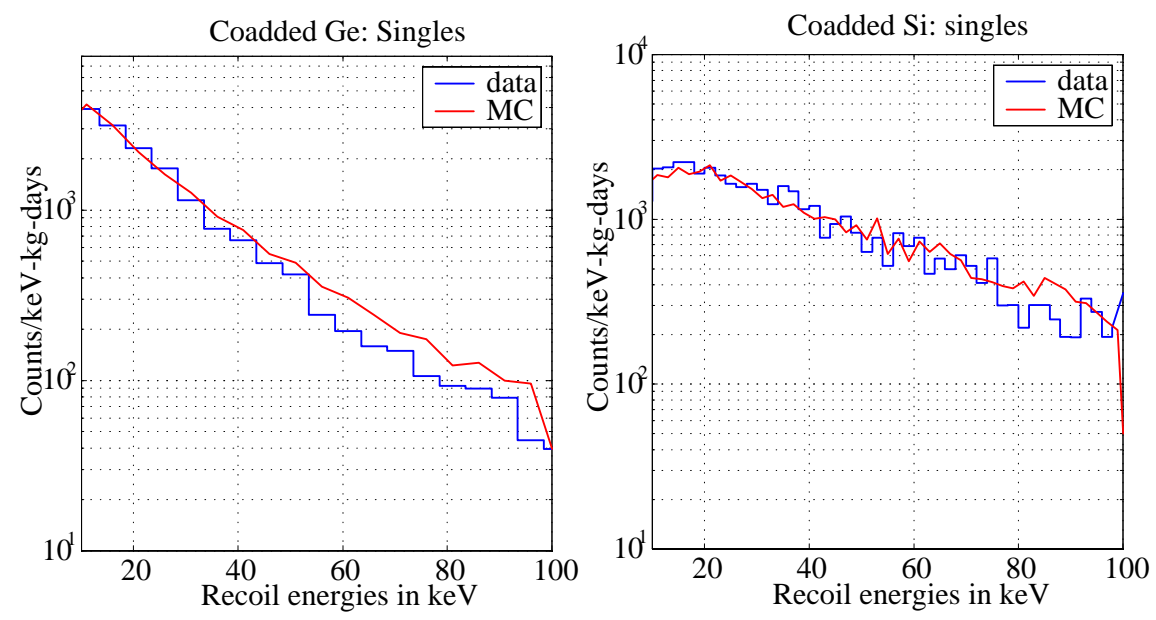

Co-added Ge and Si detectors: single-scatter events.

\subsubsection{Maximum Likelihood Analysis}

\section{V Neutron Calibration}

As explained in Chapter III, the Run 21 data analysis proceeded along two tracks: an initial analysis of just the $3 \mathrm{~V}$ data alone[13] and the combined $3 \mathrm{~V}$ and $6 \mathrm{~V}$ analysis[14]. The principal difference between the two analyses was in the discrimination techniques applied to the low-yield beta events that leak into the signal region and may be mistaken for neutrons or WIMPs. While the first analysis used 


\begin{tabular}{|c|c|c|c|c|}
\hline Evts/kg/day & All events(data) & All events(MC) & Singles(data) & Singles(MC) \\
\hline Z1 & $4.45 \pm 0.10 \times 10^{4}$ & $4.86 \pm 0.05 \times 10^{4}$ & $3.32 \pm 0.09 \times 10^{4}$ & $3.55 \pm 0.04 \times 10^{4}$ \\
\hline Z2 & $4.13 \pm 0.09 \times 10^{4}$ & $4.75 \pm 0.05 \times 10^{4}$ & $2.49 \pm 0.07 \times 10^{4}$ & $2.78 \pm 0.04 \times 10^{4}$ \\
\hline Z3 & $3.49 \pm 0.08 \times 10^{4}$ & $4.11 \pm 0.05 \times 10^{4}$ & $1.77 \pm 0.06 \times 10^{4}$ & $2.23 \pm 0.03 \times 10^{4}$ \\
\hline Z4 & $9.39 \pm 0.20 \times 10^{4}$ & $9.41 \pm 0.11 \times 10^{4}$ & $4.74 \pm 0.16 \times 10^{4}$ & $4.92 \pm 0.08 \times 10^{4}$ \\
\hline Z5 & $3.01 \pm 0.08 \times 10^{4}$ & $3.22 \pm 0.04 \times 10^{4}$ & $1.56 \pm 0.06 \times 10^{4}$ & $1.84 \pm 0.03 \times 10^{4}$ \\
\hline Z6 & $6.63 \pm 0.19 \times 10^{4}$ & $6.97 \pm 0.10 \times 10^{4}$ & $4.09 \pm 0.15 \times 10^{4}$ & $4.46 \pm 0.08 \times 10^{4}$ \\
\hline
\end{tabular}

Table 5.2: Event rates for all events and single scatter events detector-wise for the Run 21 3V neutron calibration. The efficiencies of the standard 3V cuts have been folded into the Monte Carlo simulations.

a flat cut in the rise time-yield plane to reject this background, the more extended study involved a maximum likelihood analysis of the possible beta contamination of the signal region.

The first analysis of the Run $213 \mathrm{~V}$ data constituted the thesis work of Tarek Saab. The analysis of the extended data set with the likelihood analysis was part of the dissertation of Don Driscoll. The use of maximum likelihood methods to estimate the beta leakage into the signal region was first developed by Vuk Mandic. The analysis techniques were further developed and fully applied to the entire Run 21 data set by Don Driscoll. My work involved extending this work to the neutron simulations to give them the same structure as the data in terms of the likelihood analysis. To the simulations, we sought to apply the efficiencies of the maximum likelihood ratio cuts in the data so as to be able to do a true comparison of data and modelled for the various calibration cases.

For the $(3 \mathrm{~V}+6 \mathrm{~V})$ analysis, a new cut was formulated that used the rise time, yield and recoil energy associated with each event to separate out the low-yield betas and neutrons in the various calibration data populations. The fiducial-volume of a detector was also redefined, accepting events that were $5 \sigma$ above the noise in the inner charge electrode region and consistent with noise in the outer region[19]. 
This cut was more restrictive in that it did not accept events shared between the two electrodes. The fiducial-volume cut takes into account the energy dependence in the cross-talk between the inner and outer electrodes. This cross-talk is both detector and bias voltage dependent.

The simulations of the calibration runs at the two voltages provided a means of verifying that the data cuts were well understood. Given below is a summary of the comparisons of data and Monte Carlo output for the 3V neutron calibrations using the maximum likelihood analysis cuts.

From the nuclear-recoil energies provided by the simulations, the charge energy distribution and the yield were quantified as explained in Section 5.4.1. The noise in the outer electrode was remodelled to take into account the energy dependence of the cross-talk in the data and the fiducial-volume cut specified for the Monte Carlo output[19]. A rise time distribution for the simulated neutrons was modelled based on fits provided by the data.

The rise time, yield and recoil energies of the events were then used to compute the likelihood function ratio for the distribution. Figure 5.7 shows a histogram of the maximum likelihood function ratio distribution for both data and simulations for detector Z2. The likelihood function cut selects all events to the left of the solid vertical line.

Events were defined as single-scatters when they passed all cuts, including the likelihood function ratio cut for the detector in question, while all other detectors had less than $2 \mathrm{keV}$ energy deposition in them.

Figure 5.8 shows the comparison of data and simulations for single-scatter events seen in the $3 \mathrm{~V}$ neutron calibration. In the data, the maximum likelihood cut was formulated for the single-scatter events to estimate the neutron survival percentage in the single-scatter events. The low neutron survival percentage for the two silicon detectors as well as for the germanium detector Z1 made it necessary to exclude them from the analysis [14]. Only the three germanium detectors 


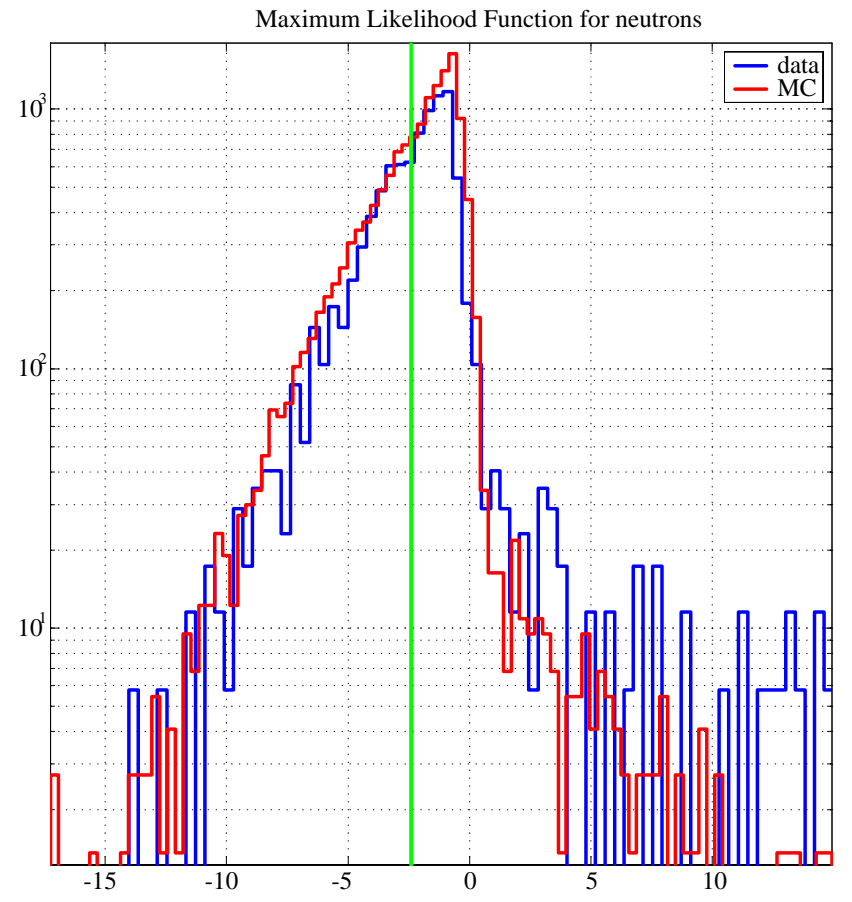

Figure 5.7: Maximum likelihood distribution function for data and simulations for the case of detector Z2. The solid green vertical line shows the cut. Events to the left of the line are likely neutrons according to the data distribution. Details of the analysis are in Chapter III 


\begin{tabular}{|c|c|c|}
\hline Evts/kg/day & Single scatters (data) & Single scatters (MC) \\
\hline Z2 & $(8.8 \pm 0.4) \times 10^{3}$ & $(11.0 \pm 0.2) \times 10^{3}$ \\
\hline Z3 & $(6.9 \pm 0.4) \times 10^{3}$ & $(9.5 \pm 0.2) \times 10^{3}$ \\
\hline Z5 & $(3.6 \pm 0.3) \times 10^{3}$ & $(4.5 \pm 0.1) \times 10^{3}$ \\
\hline
\end{tabular}

Table 5.3: Event rates for the Neutron Calibration using the Maximum Likelihood Ratio cuts. The table shows the single scatter events for the three germanium detectors Z2, Z3 and Z5.

Z2, Z3 and Z5 were considered for the comparisons. Table 5.3 compares rates in events $/ \mathrm{kg} /$ day for single-scatters in these three detectors for data and simulations. As can be seen for the spectra and rates, the modelled output shows a slight overestimate as compared to data over the entire energy range. This is suggestive of the fact that limited statistics in the distributions used to define the likelihood fitting functions could be the cause of the discrepancy in fitting the same functions to a modelled population of neutrons. The variation between data and simulation as a consequence of such statistical fluctuations were taken into account by making a conservative estimate of the efficiencies to be applied to the external neutron simulation for the neutron subtraction necessary for the dark matter analysis. This procedure has been explained in Sections 6.4.1 and 6.4.2

One of the principal motivations for the use of the maximum likelihood analysis method was to optimize the characterization of the beta contamination in the population of events that show double-scatters in neighboring detectors. As multiple-scatter events such as nearest-neighbor scatters are used to quantify and subtract the limiting neutron background in the final dark matter analysis, it is critical to ensure that the population consists of nuclear-recoil events and not due to leakage of betas into the signal region.

The beta events may arise due to surface electrons arising from contamination of the detectors or due to 'ejectrons'. The latter are ejected electrons that occur when photons scatter in neighboring detectors. These electrons in turn give 


\begin{tabular}{|c|c|c|}
\hline Events passing cuts/Raw LiveDays & Data & Simulations \\
\hline All Singles in Z2/3/5 & $(4.84 \pm 0.17) \times 10^{3}$ & $(6.41 \pm 0.09) \times 10^{3}$ \\
\hline Nearest Neighbor Doubles & $(0.82 \pm 0.07) \times 10^{3}$ & $(1.13 \pm 0.04) \times 10^{3}$ \\
\hline Non-nearest Doubles & $(1.16 \pm 0.08) \times 10^{3}$ & $(0.90 \pm 0.03) \times 10^{3}$ \\
\hline Triples & $(0.56 \pm 0.05) \times 10^{3}$ & $(0.43 \pm 0.02) \times 10^{3}$ \\
\hline
\end{tabular}

Table 5.4: Event rates for data and simulations for single and multiple scatter events in the $3 \mathrm{~V}$ Neutron Calibration

rise to surface scatters with low-yield that can be mistaken for nuclear-recoil events.

The extended analysis applied a maximum likelihood fit to discriminate against the betas in this population. Chapter III explained the details of the analysis techniques used. In this chapter, we present comparisons of the rates of nearest-neighbor double-scatters in the data and simulated event populations.

double-scatters that occur in non-nearest-neighbor detectors are not likely to be contaminated by low-yield betas. low-yield events that have three or more scatters are likely to be bona-fide nuclear-recoil events since such random events are infrequent and electrons are not likely to have that much penetration to be able to produce such scatters. For the population of non-nearest-neighbor doubles and triples, the analysis used the flat rise time cut to discriminate against lowyield events. Triples were identified as events for which at least three detectors had energies above the phonon energy threshold. The non-nearest-neighbor required two detectors to have energies above threshold and both detectors pass the data quality and fiducial-volume cut.

Table 5.4 compares rates in number of events/Raw Live Days for the various populations of multiple-scatter events seen in the data and simulations using both the standard rise time and the maximum likelihood analysis cuts.

Again, as may be seen from Tables 5.3 and 5.4, there is a slight overestimate in the event rates from the simulated output. This variation between data 
and Monte Carlo simulations was quantified for both the calibration and internal neutron event population, as shown in Section 6.4.1 and Section 6.4.2. This was used to make a conservative estimate of the efficiencies applied to the simulations of the limiting neutron background. This procedure has been detailed in Chapter VI.

\section{V Neutron Calibration}

While data for the silicon detectors was taken at $4 \mathrm{~V}$ ionization voltage throughout the run, the germanium detectors were operated at $3 \mathrm{~V}$ and $6 \mathrm{~V}$ charge biases. Operating at the two voltages helped decide the optimal bias for the WIMP-search data. While $6 \mathrm{~V}$ charge bias improves the yield for events arising from surface scatters of betas, operating at $3 \mathrm{~V}$ provided a better means of discrimination based on the rise time of the phonon pulse [15]. The maximum likelihood analysis was also applied to the 6V WIMP-search and calibration data. Cuts formulated based on the $6 \mathrm{~V}$ neutron calibration data were studied using the simulation of the calibration run in a manner similar to the $3 \mathrm{~V}$ data. A $6 \mathrm{~V}$ neutron calibration run with the ${ }^{252} \mathrm{Cf}$ source placed to the side of the shield was used as the basis for the comparison. Figure 5.9 shows the comparison of spectra for the single-scatter events for the $6 \mathrm{~V}$ Neutron calibration run. As before, the likelihood cuts are formulated for the simulations from the data representation. Table 5.5 and 5.6 compare the single and multiple-scatter rates for data and simulations for the $6 \mathrm{~V}$ calibration run. The rates for the simulated output are higher than the data which suggests that limited statistics in the distributions used to model the likelihood fitting functions may be the cause of the discrepancy. Again, the systematically higher rate of the simulations was accounted for by making a conservative estimate of the efficiencies of the calibration and veto-coincident cases, as has been explained in Chapter VI. There is also a considerable overestimate in the simulated event rate for the nearest-neighbor double events in the $6 \mathrm{~V}$ neutron calibration. This too was accounted for by making a conservative estimate on the efficiencies so as to ensure 

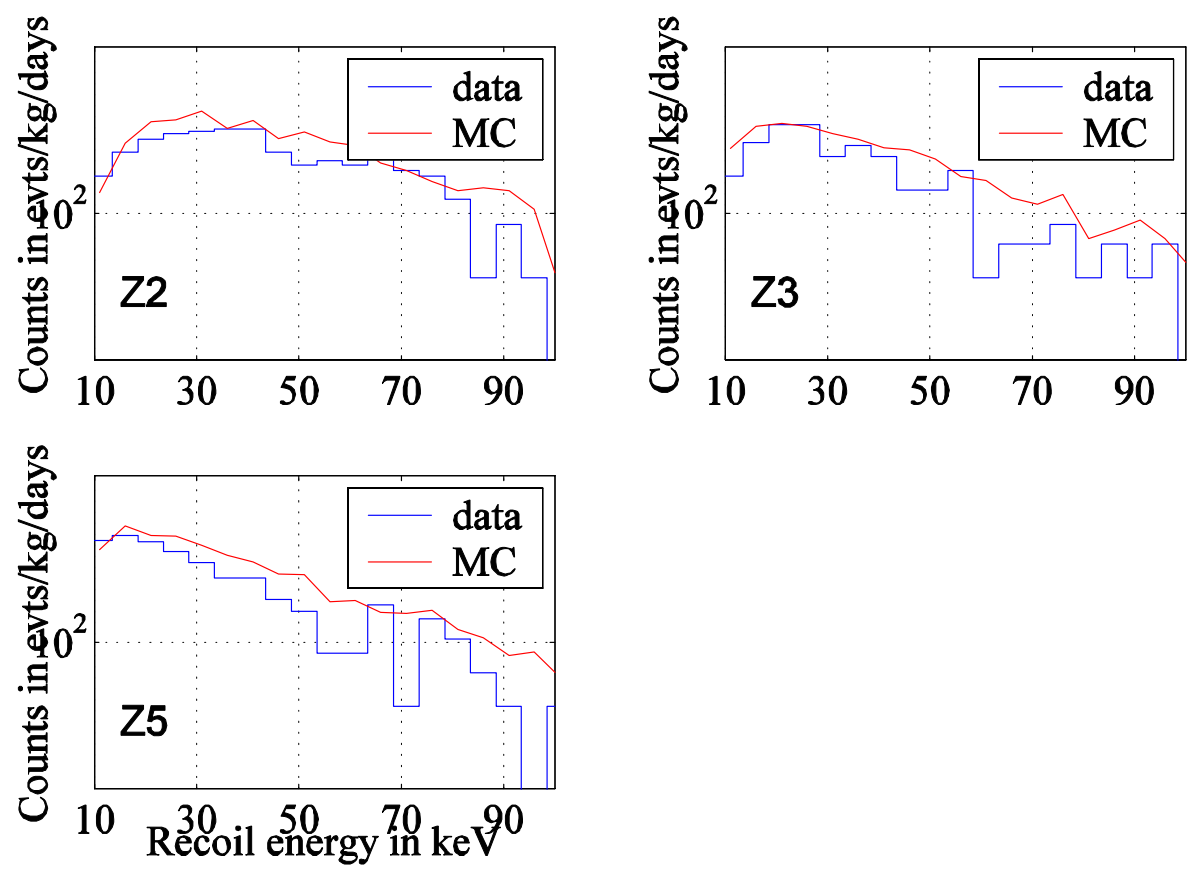

Figure 5.8: Comparison of spectra for data and simulation for the $6 \mathrm{~V}$ Neutron Calibration Run. The comparison uses the cuts defined by the maximum likelihood ratio analysis for comparing spectra with the simulated output for Z2, Z3 and Z5.

that the number of doubles were not overestimated in the neutron subtraction for the dark matter analysis.

\subsection{Muon coincident nuclear-recoil events}

In Run 21, of the 4.7 million events recorded with a $3 \mathrm{~V}$ ionization voltage bias, 3.6 million events occurred within $40 \mu \mathrm{s}$ of preceding activity in the muon scintillator veto, and are characterized as muon-coincident events[13]. Given the relatively shallow depth of the site, and consequently the high muon rate measured here, this is a particularly substantial population. Over $80 \%$ of the $\sim 50,000$ events that occurred daily during the WIMP-search data were a consequence of the muon flux $[13]$. 


\begin{tabular}{|c|c|c|}
\hline Evts/kg/day & Single scatters (data) & Single scatters (MC) \\
\hline Z2 & $(5.4 \pm 0.4) \times 10^{3}$ & $(6.9 \pm 0.2) \times 10^{3}$ \\
\hline Z3 & $(3.8 \pm 0.3) \times 10^{3}$ & $(5.4 \pm 0.2) \times 10^{3}$ \\
\hline Z5 & $(5.5 \pm 0.3) \times 10^{3}$ & $(7.4 \pm 0.2) \times 10^{3}$ \\
\hline
\end{tabular}

Table 5.5: Event rates for the 6V Neutron Calibration using the Maximum Likelihood Ratio cuts. Shown are the event rates for the single scatter events in Z2, Z3 and Z5.

\begin{tabular}{|c|c|c|}
\hline Events passing cuts/Raw LiveDays & Data & Simulations \\
\hline All Singles in Z2/3/5 & $(3.70 \pm 0.16) \times 10^{3}$ & $(4.93 \pm 0.08) \times 10^{3}$ \\
\hline Nearest Neighbor Doubles & $(0.39 \pm 0.01) \times 10^{3}$ & $(0.93 \pm 0.03) \times 10^{3}$ \\
\hline Non-nearest Doubles & $(0.53 \pm 0.06) \times 10^{3}$ & $(0.93 \pm 0.03) \times 10^{3}$ \\
\hline Triples & $(0.45 \pm 0.02) \times 10^{3}$ & $(0.55 \pm 0.06) \times 10^{3}$ \\
\hline
\end{tabular}

Table 5.6: Event rates for data and simulations for single and multiple scatter events in the 6V Neutron Calibration. Table shows rates of singles for detectors Z2, Z3 and Z5 and the rates of nearest-neighbor doubles, non-nearest neighbor doubles and triples for all detectors. The last two populations are defined using the flat rise time cut. 
Neutrons produced by interactions of cosmic ray muons with the material of the shield, principally the copper and lead layers, feature in this data set. As nuclear-recoils produced by them occur within $40 \mu \mathrm{s}$ of activity in the muon scintillator veto, they do not feature in the dark matter analysis. They, however, serve as another means of calibration as they occur over the entire period of the run. By providing a large population of nuclear-recoil events, this "internal" neutron set is an important measure of the stability of the detectors.

Chapter III gave specifics of the various event populations seen in coincidence with a trigger in the muon veto. This section details how veto-coincident events seen in the nuclear-recoil band may be used as another calibration measure to check the simulations.

\subsection{Simulating the Veto-coincident Neutrons}

Neutrons produced by muon interactions with the shield provide an important background. While these events are vetoed out of the dark matter analysis, their occurrence over the period of a run allows their use in monitoring the stability of the cut efficiencies.

At the $17 \mathrm{~m}$.w.e depth of SUF, the hadronic component of the muon flux is negligible. Neutrons arise principally from muon capture from slow muons or from low-energy photo-nuclear interactions or photo-fission interactions inside the shield. With the integrated muon flux at this site being $44 \mathrm{muons} / \mathrm{m}^{2} / \mathrm{s}[16]$, this provides a large sample population of nuclear-recoil events on which to further test the modelling of the neutron backgrounds.

Though muon interactions occur in the various shield layers, we consider principally the contribution from the lead and copper layers. Neutrons produced in the outer lead are easily moderated by the outer polyethylene and constitute around $3 \%$ of the total rate[5]. Neutrons produced in the outer polyethylene are 

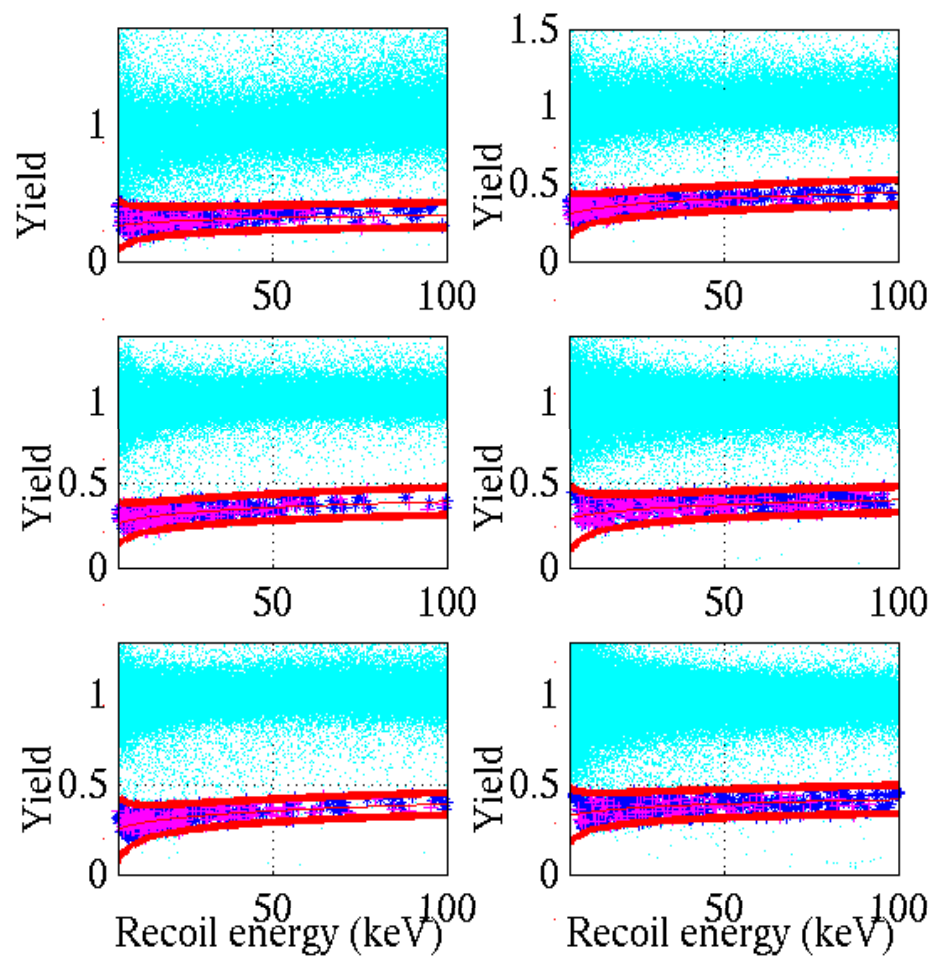

Figure 5.9: Yield vs Recoil for the 3V Muon coincident Low Background data set. The bands show events in the signal region passing the fiducial volume and rise time cut. The blue asterix indicates a single scatter while magenta crosses indicate events that multiply scatter in two or more detectors. 
also sufficiently suppressed by the passive shielding. The main contribution comes from the inner lead layer and the copper cans of the icebox.

The neutron production rate from muon capture and electromagnetic pro-

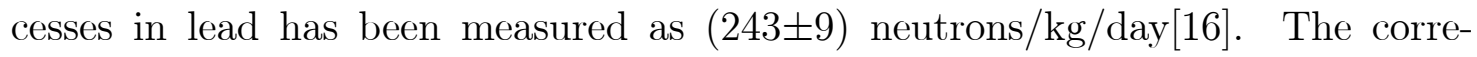
sponding rate for copper has been estimated as 100 neutrons/kg/day[16]. Based on measured production rates in lead and estimates in copper, the neutron rate has been estimated to be $1 \times 10^{4}$ /day from low-energy photo-nuclear interactions and $3.2 \times 10^{4}$ /day from muon capture in the inner lead and copper cans[17].

As explained at the beginning of the chapter, GEANT3.2 is not used to simulate the neutron production processes. Instead production rates of the neutrons in different material, as available from direct measurements or literature, are used as the inputs. In Chapter IV, we considered the various neutron production mechanisms and the spectral distribution of the neutrons produced. Neutrons produced by muon capture in lead follow the spectrum described by[16]

$$
\frac{d N}{d E}=\left\{\begin{array}{c}
0.812 E^{5 / 11} e^{\left(-\frac{E}{1.22}\right)} \text { for } \mathrm{E}<4.5 \mathrm{MeV} \\
0.018 e^{\frac{-E}{9}} \text { for } \mathrm{E}>4.5 \mathrm{MeV}
\end{array}\right\}
$$

The low-energy component of neutrons produced in electromagnetic and nuclear showers can be modelled by an energy spectrum similar to that of muon capture.

high-energy neutrons have low interaction cross sections in polyethylene and can punch through it easily to interact with other components of the shield and produce secondaries that cause recoils in the detectors. However, neutrons of high energies produced in the inner lead and copper cans do not have layers of polyethylene separating them from the detectors and do not provide as significant a contribution $(\sim 5 \%)$ to the event rate as the lower energy component. The rate of such neutrons produced in the material within the polyethylene is $\sim 4 / \mathrm{kg} / \mathrm{day}[5]$. This contribution is small in comparison to the more dominant rates $(\sim 100 / \mathrm{kg} /$ day 
in copper and $243 / \mathrm{kg} /$ day in lead) due to muon capture. Besides such neutrons have relatively small interaction cross-sections in the detectors.

high-energy neutrons produced in the outer lead may punch through the outer polyethylene in the manner described earlier. It is also estimated that $40 \%$

of the high-energy neutrons produced in the rock of the experimental cavern[18] trigger the veto and so find inclusion in the population of veto-coincident events. However, these contribution is small compared to the much larger event rate from muon capture neutrons in the veto coincident population. The high-energy part of the spectrum is not modelled in the simulations as the higher energy neutrons have a very small production rate as compared to the lower energy components and the contribution of the latter dominates the event rate in the detectors as was explained earlier.

The simulations also do not model the photons produced along with the neutrons in the muon induced processes. Photons produced from capture of neutrons of thermal energies in the polyethylene are also not propagated in the simulations. The only gammas that are propagated are secondaries produced by interactions of the neutrons with the material of the shield. Events with nuclear and electron-recoils occur in the same detector are thus underestimated by the Monte Carlo simulations. In the data, the reduction in the nuclear-recoil efficiency due to such events is less than $2 \%$ [5]. The simulations may thus slightly overestimate the number of single-scatter nuclear-recoil events seen during a run. The spectral distribution for neutrons produced in the copper is taken to be similar to lead, following the functional form described by Equation 5.2.

\subsubsection{First analysis using the flat rise time cut}

Efficiencies of the data cuts were applied to the simulated output of the internal neutrons is a manner similar to that of the neutron calibration data set. The ionization energies and the fiducial-volume cut were modelled as was done for the case of the calibration neutrons in Section 5.4.1. 
For the veto-coincident nuclear-recoil event populations, a cut on the rise time of the phonon pulse is made to ensure the non-inclusion of low-yield surface events that may be mistaken for nuclear-recoils. The rise time cut accepts events with a phonon pulse rise time of $12 \mu s$ for the germanium and $6 \mu s$ for the silicon detectors. The cut rejects $80 \%$ of the surface electron-recoils over the energy range of $10-100 \mathrm{keV}$ while accepting $50 \%$ of the bona-fide nuclear-recoil scatters[13].

The energy dependent efficiencies of the rise time cut are folded into the simulation output. Singles are identified as events passing all cuts including the fiducial-volume and rise time cut while the multiple cut requires at least one of the detectors pass the rise time cut.

Figure 5.11 shows the comparison of data and simulations for all events in the 10 - $100 \mathrm{keV}$ range in the 3V muon-coincident low background data set. Figure 5.12 shows the comparison of single-scatter events for the same energy range. There is good agreement between data and simulations confirming our understanding of the modelling of the neutron backgrounds. The event rates for the single-scatter events in the germanium and silicon detectors also show a good agreement for the data and simulations allowing us to confirm that the simulations are true to the experimental conditions. The spectra of the single-scatter events are shown separately as it is this population in the germanium detectors that provides the WIMP-search data in the veto anti-coincident nuclear-recoil event data set. 

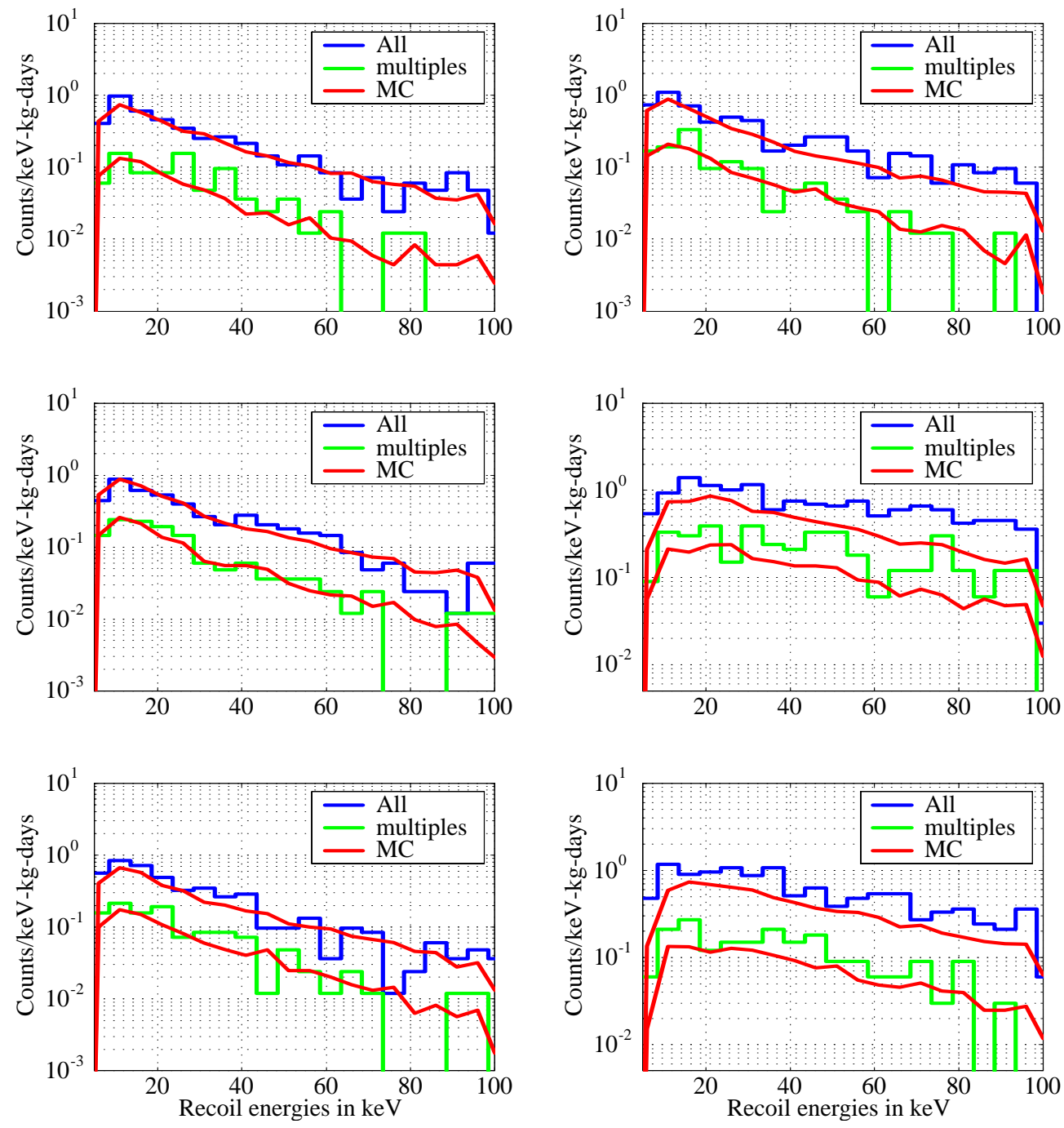

Figure 5.10: Comparison for data and simulation for all (upper curves) and multiple scatter events (lower curves) for the 3V Muon Coincident Low Background Data. The plots show the six detectors from top left to bottom right. 

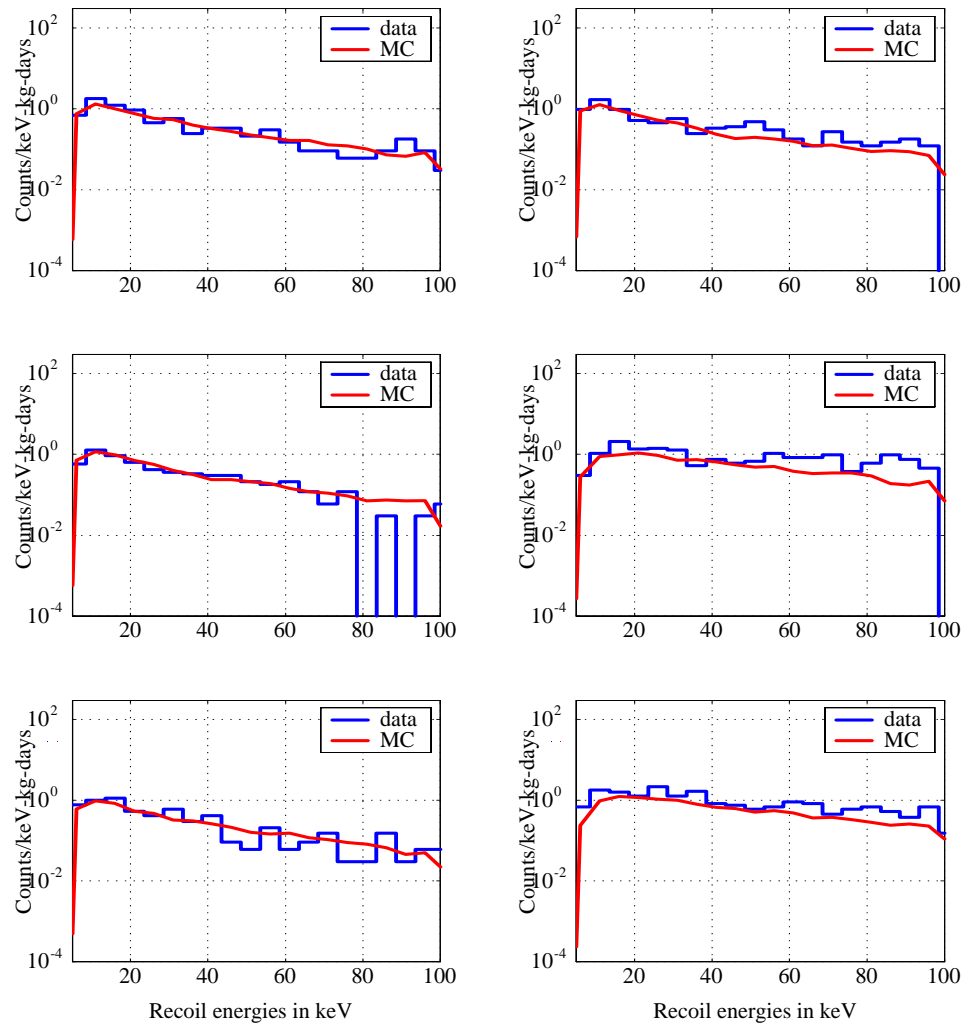

Figure 5.11: Comparison for data and simulation for single scatter events for the 3V Muon Coincident Low Background Data. The plots show the six detectors from top left to bottom right. 

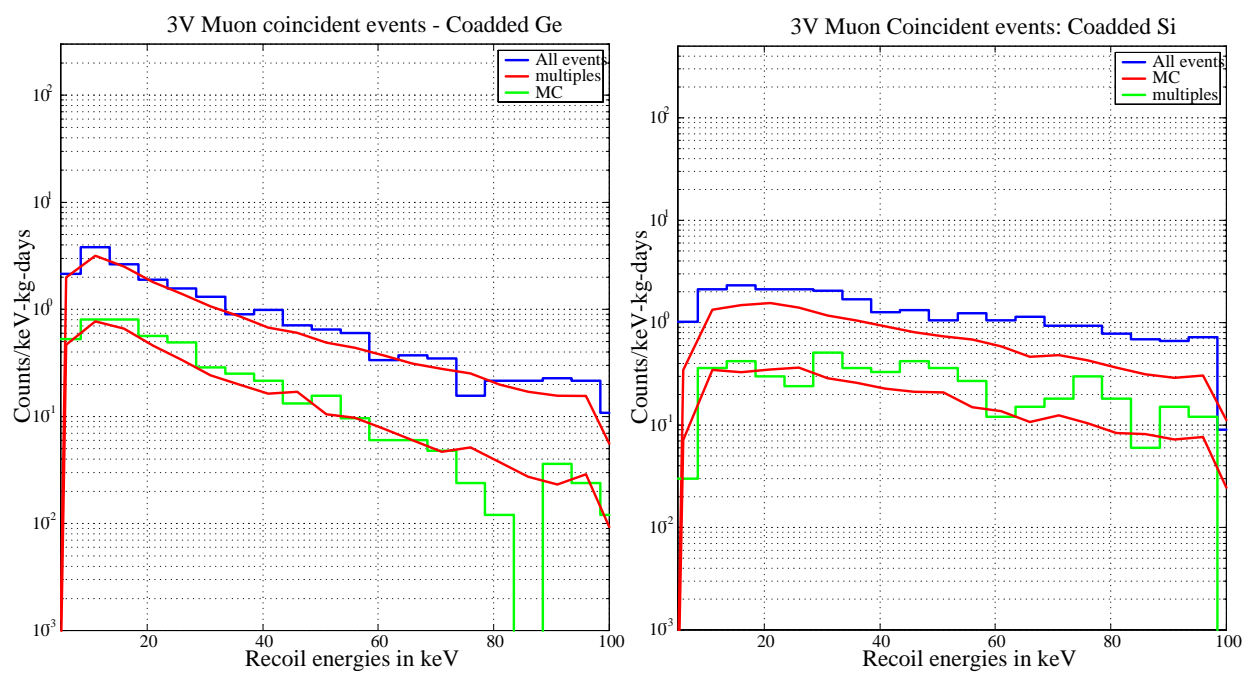

Co-added Ge and Si detector event rates for all and multiple-scatter events
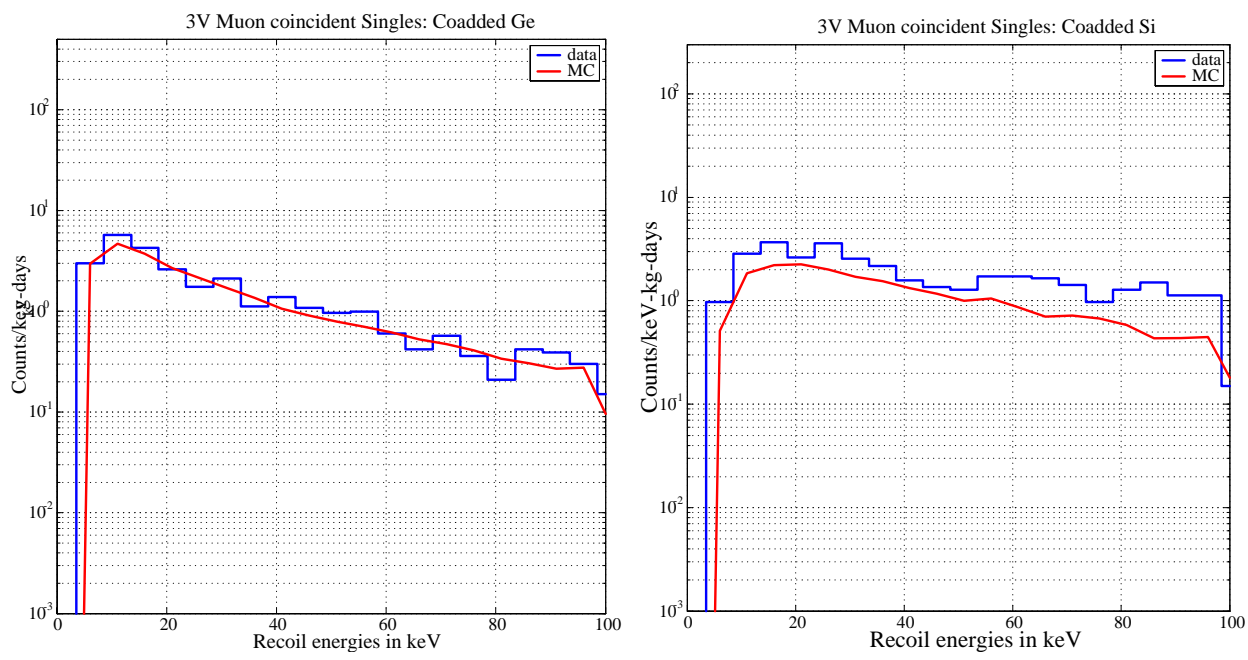

Co-added Ge and Si detector event rates for single-scatter events

\subsubsection{Maximum Likelihood Analysis}

\section{V Muon Coincident data}

In Section 5.4.2 we discussed the application of the maximum likelihood ratio cuts to the neutron calibration data and the comparisons of data and simulations 


\begin{tabular}{|c|c|c|}
\hline & Data & MC \\
\hline Singles rate in evts/kg/day & $(17.73 \pm 0.47)$ & $(17.25 \pm 0.15)$ \\
\hline Ge singles rate evts/kg/day & $(14.20 \pm 0.46)$ & $(14.69 \pm 0.15)$ \\
\hline Si singles rate evts/kg/day & $(35.26 \pm 1.63)$ & $(30.06 \pm 0.48)$ \\
\hline Si singles/Ge singles & 0.49 & 0.41 \\
\hline
\end{tabular}

Table 5.7: Event rates for single scatter events in the four germanium and two silicon detectors for the Run21 3V Veto Coincident data. The efficiencies of the standard 3V cuts have been folded into the Monte Carlo simulations.

for the rates and spectra of these neutrons. The same procedure is followed for the veto coincident data set. As before rise time, yield and recoil energies are computed for every event in the simulation and its likelihood ratio computed. A cut is applied selecting single-scatter events in the three germanium detectors Z2, Z3 and Z5. Events passing the fiducial-volume and likelihood ratio cuts which single-scatter in one detector are shown in Figure 5.19. As explained earlier, only the three germanium detectors are considered in this analysis. Table 5.8 shows the comparisons of the rates of singles for data and simulations. Good agreement is seen between data and simulations. Table 5.9 shows comparison of rates for all events passing the cuts and multiple-scatter nuclear-recoil events in the veto coincident data set. The nearest-neighbor double-scatters were analyzed using the maximum likelihood estimate method as this population may include events arising from surface beta contamination of the signal region. The non-nearest-neighbors and triples were examined using the standard rise time cut.

\section{V Muon Coincident Data}

A similar analysis was done for the 6V Muon Coincident Low Background Data. Figure 5.20 shows the comparison of spectra while Table 5.10 and 5.11 compare rates for the various event populations in the $6 \mathrm{~V}$ data using the maximum 

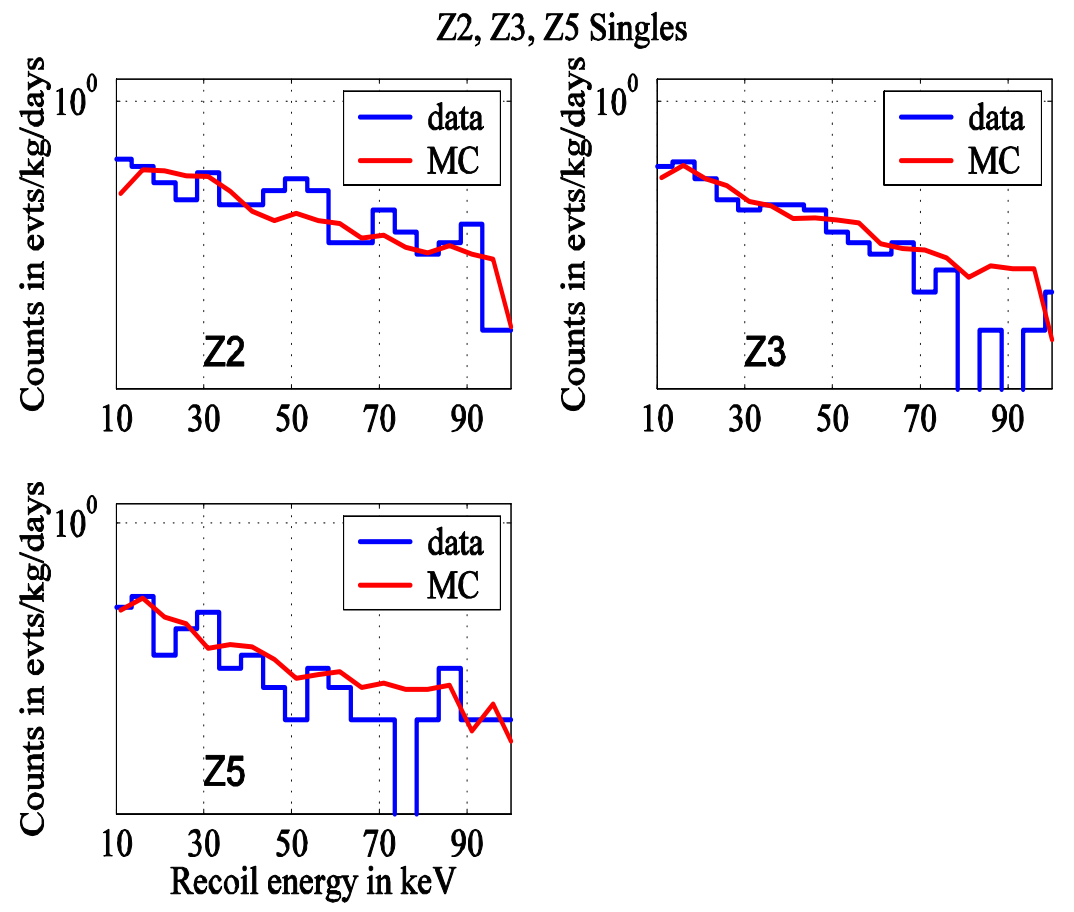

Figure 5.12: Single scatter nuclear recoil spectra in Z2, Z3 and Z5 for the veto coincident nuclear recoil event population. The data and simulations are in reasonable agreement. The rates are in event $/ \mathrm{kg} /$ days.

\begin{tabular}{|c|c|c|}
\hline Evts/kg/day & Single scatters (data) & Single scatters (MC) \\
\hline Z2 & $14.24 \pm 1.03$ & $13.18 \pm 0.29$ \\
\hline Z3 & $10.09 \pm 0.86$ & $11.19 \pm 0.27$ \\
\hline Z5 & $5.19 \pm 0.62$ & $4.5 \pm 0.19$ \\
\hline
\end{tabular}

Table 5.8: Event rates for the 3V Veto Coincident Neutrons using the Maximum Likelihood Ratio cuts. 


\begin{tabular}{|c|c|c|}
\hline Events passing cuts/Raw LiveDays & Data & Simulations \\
\hline All Singles in Z2/3/5 & $3.70 \pm 0.16$ & $7.56 \pm 0.11$ \\
\hline Nearest Neighbor Doubles & $0.57 \pm 0.10$ & $1.16 \pm 0.04$ \\
\hline Non-nearest Doubles & $1.26 \pm 0.15$ & $0.95 \pm 0.04$ \\
\hline Triples & $0.59 \pm 0.10$ & $0.43 \pm 0.03$ \\
\hline
\end{tabular}

Table 5.9: Event rates for data and simulations for single and multiple scatter events in the $3 \mathrm{~V}$ Veto Coincident data set.

\begin{tabular}{|c|c|c|}
\hline Evts/kg/day & Single scatters (data) & Single scatters (MC) \\
\hline Z2 & $(11.67 \pm 1.06)$ & $(9.22 \pm 0.24)$ \\
\hline Z3 & $(6.98 \pm 0.82)$ & $(6.79 \pm 0.21)$ \\
\hline Z5 & $(10.42 \pm 0.99)$ & $(9.38 \pm 0.24)$ \\
\hline
\end{tabular}

Table 5.10: Event rates for the 6V Veto Coincident Neutrons using the Maximum Likelihood Ratio cuts. Table shows the single scatter event rates in the three germanium detectors considered.

likelihood analysis cuts. Unlike the case of the $3 \mathrm{~V}$ data, there is not as good an agreement between the data and the simulations for the single-scatter rates and spectra. The recoil spectra for the single-scatters in the three germanium detectors are given in Figure 5.20.

\subsection{Use of the simulations}

This chapter discussed the method by which neutron backgrounds were simulated for Run 21. It also showed how inputs from the data serve as checks on the simulations and how comparison of rates and spectra need be done to ensure that the efficiencies of the data cuts are applied correctly to the simulated output. The process of applying cut efficiencies to the population of events generated 

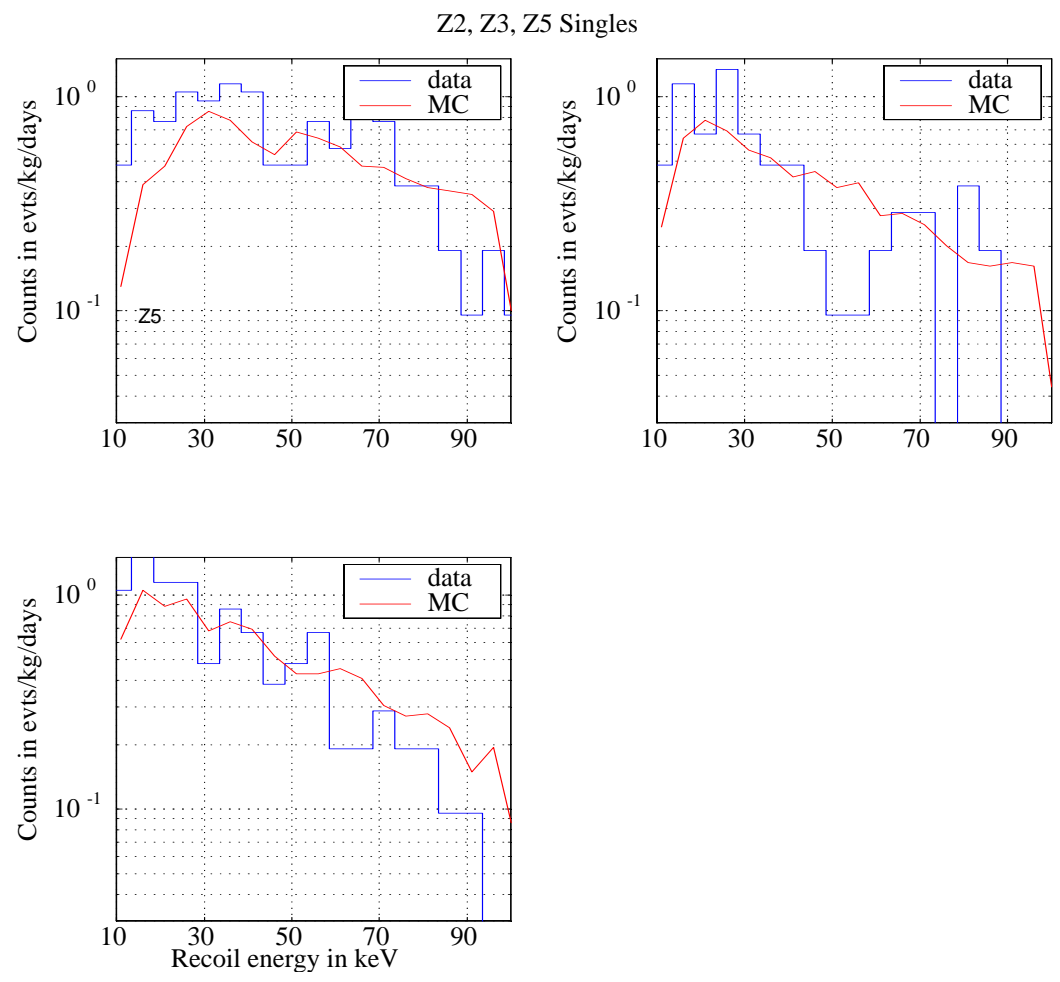

Figure 5.13: Recoil energy spectra for the single scatter events in Z2, Z3 and Z5 for the $6 \mathrm{~V}$ veto coincident neutrons. The simulations show a slight overestimate in the efficiencies over the energy range of $10-100 \mathrm{keV}$.

\begin{tabular}{|c|c|c|}
\hline Events passing cuts/Raw LiveDays & Data & Simulations \\
\hline All Singles in Z2/3/5 & $7.27 \pm 0.42$ & $6.35 \pm 0.10$ \\
\hline Nearest Neighbor Doubles & $0.57 \pm 0.12$ & $0.91 \pm 0.04$ \\
\hline Non-nearest Doubles & $1.46 \pm 0.19$ & $1.00 \pm 0.04$ \\
\hline Triples & $0.71 \pm 0.13$ & $0.44 \pm 0.44$ \\
\hline
\end{tabular}

Table 5.11: Event rates for data and simulations for single and multiple scatter events in the $6 \mathrm{~V}$ Veto Coincident data set. 
by a Monte Carlo run is then applied to the simulation of the external neutron background to provide the necessary inputs for the WIMP-search analysis. The procedure is explained in the next chapter.

The comparison of data and simulations for the first analysis of the $3 \mathrm{~V}$ data showed good agreement allowing for applying the efficiencies of the data cuts to the external neutron simulation in the same manner as was done in the case of the vetocoincident and calibration neutrons. For the analysis of data using the maximum likelihood analysis cuts, the disagreement between the recoil spectra in the case of the data and modelled output for the calibration and internal neutron case made it necessary for use to make a conservative estimate of the efficiencies of the cuts. The efficiencies were thus estimated from the two cases and the conservative value chosen applied to the external neutron Monte Carlo for the background subtraction necessary for the WIMP analysis. The procedure to estimate the limits given the variation in efficiencies has been outlined in Chapter VI. 


\section{References}

[1] GEANT Version 3.21.04, GEANT User's Guide, CERN, Geneva (1995).

[2] H. C. Fesefeldt III, Gheisha version 7.03 (1985).

[3] J. O. Johnson and T. A. Gabriel, MICAP: A Monte Carlo Ionization Chamber Analysis Package (1993).

[4] A. Fasso et al, Proc. of the $4^{\text {th }}$ Intl. Conf. on Calorimetry in high-energy Physics, 493 (1993).

[5] T. A. Perera, Ph.D. Thesis, Case Western Reserve University (2002), unpublished.

[6] PAW Version 2.13/08, Physics Analysis Workstation Reference Manual, CERN, Geneva (2002).

[7] E. J. Axton and A. G. Bardell, Metrologia, 21, 59 (1985).

[8] D.H. Stoddard and H.E. Hootman, ${ }^{252}$ Cf Shielding Guide, Savannah River Laboratory Internal Report, DP-1245 (1971).

[9] W. Rau, CDMSNote 0206004 (2003).

[10] R. C. Reedy, J. R. Arnold and D. Lal, Ann. Rev. Nucl. Part. Sci., 33, 505 (1983).

[11] Table of Radioactive Isotopes, http://nucleardata.nuclear.lu.se 
[12] E Segre, Nuclei and Particles, Second Edition W A Benjamin Inc. (1977).

[13] T. Saab, Ph.D. Thesis, Stanford University (2002), unpublished.

[14] D. D. Driscoll, Ph.D. Thesis, Case Western Reserve University (2004), unpublished.

[15] D. S. Akerib et al., Phys. Rev. D, 68, 082002 (2003).

[16] A. da Silva, Ph. D. Thesis, The University of British Columbia, (1996).

[17] S. R. Golwala, Ph.D. Thesis, University of California at Berkeley (2000), unpublished.

[18] S. Yellin, private communication.

[19] D.D. Driscoll, Run 21 Ebook Note 305 (2003). 


\section{Chapter 6}

\section{Improved WIMP Limits at the Shallow Site}

A limiting neutron background due to high-energy neutrons generated from muon-induced processes in the rock at the Stanford Underground Facility necessitated the move deeper underground to Soudan. The presence of this background had been indicated by the results of Run 19, the earlier WIMP-search run at this facility. Run 21 served to confirm that the events seen after all cuts were consistent with a neutron background, as well as demonstrating the performance and scientific reach of these new ZIP detectors in anticipation of the Soudan phase of the experiment.

The simulation of the external neutron background seen at the shallow site was used to predict the ratios of single-scatter events in the germanium detectors (possible WIMP or neutron candidates) to single-scatter events in the silicon detectors and multiple-scatter events in all the detectors (neutrons). As noted in Section 4.1.1, WIMPs have a higher interaction rate per kilogram in germanium as compared to silicon while neutrons interact more often in silicon than in germanium. Single-scatter events in the silicon detectors are thus likely to be neutrons while germanium single-scatters have a higher possibility of being WIMP-candidate 
events. This provides a means of estimating the expected number of neutron events in the single-scatter nuclear-recoil population that constitutes the WIMP-search data. After statistically subtracting this background, and accounting for leakage of high-yield electrons in the signal region, what events remain, if any, are possible WIMPs.

Chapter V showed how the calibration data may be used to inform the simulations. This chapter indicates how the simulations can, in turn, be used to inform the WIMP-search data. It outlines details of the simulations of the external neutron background at SUF and explains how the predicted ratios from the Monte Carlo were used in conjunction with the results of the analysis of the $3 \mathrm{~V}$ data to exclude new parameter space for WIMPs of masses between $8-20 \mathrm{GeV} / \mathrm{c}^{2}$ and set limits on the WIMP-nucleon cross section that were lower than previous CDMS results for higher WIMP masses[1].

The first analysis of the Run 21 data taken at 3V ionization voltage [2] involved the application of a 'flat' rise time cut to discriminate against surface electron-recoil events that leak into the signal region. The combined analysis of the $3 \mathrm{~V}+6 \mathrm{~V}$ data[3] used maximum likelihood distribution techniques to optimize the selection of bona-fide nuclear-recoil events amongst the population of single-scatter and nearest-neighbor double-scatter events in the signal region.

In the case of both the analyses, the efficiencies of the data cuts were applied to the output of the simulation of the limiting neutron background to estimate the neutron contribution to the WIMP-search data, and set new exclusion limits to the WIMP-nucleon cross section. Statistical tests helped confirm the hypothesis that events seen after all cuts in the WIMP candidate data set were consistent with a limiting neutron background. 


\subsection{Simulating the limiting neutron background}

Neutrons produced in the rock of the experimental cavern can serve as a significant background source to the WIMP-search. These neutrons may either be produced from the natural radioactivity of the rock or as a consequence of muon interactions in the material of the rock.

Alpha decay in the ${ }^{238} \mathrm{U}$ and ${ }^{232} \mathrm{Th}$ chains lead to $(\alpha, \mathrm{n})$ reactions that produce neutrons. The low-energy neutron flux also has a contribution from the spontaneous fission of ${ }^{238} \mathrm{U}$ and ${ }^{232} \mathrm{Th}$. At the shallow site, the production rate from both processes is expected to be $(34 \pm 3)$ neutrons/kg/day in rock [4]. This flux, however, is easily suppressed by the use of passive hydrocarbon shielding.

high-energy neutrons ( $>50 \mathrm{MeV}$ ) produced from muon-nuclear interactions in the material of the rock surrounding the cavern provide the limiting background to the experiment at the shallow site. While the lower energy component arising from bremsstrahlung, pair production and muon capture processes is well moderated by the outer polyethylene layers, the high-energy particles have large interactions lengths (of the order of $\sim 100 \mathrm{~cm}$ ) in the polyethylene and are able to punch through it causing scatters in the inner lead that produce secondaries which give rise to $10-100 \mathrm{keV}$ recoils in the detectors. The high-energy neutron flux has been described in detail in Chapter IV. In Chapter VIII, we will consider a greatly reduced limiting neutron background seen at the deep site and how much exposure would be necessary for the flux to begin to limit sensitivity to the detection of dark matter at this site.

The simulation of this background was performed using the GEANT3.2 particle transport code. The GEANT3 simulations do not model the production of neutrons from muon induced processes, relying on literature for a measure of the neutron flux seen at a site. Based on earlier measurements and analytical studies, the high-energy neutron distribution was modelled using [5]: 


$$
\frac{d N}{d E}=\left\{\begin{array}{c}
6.05 e^{\left(-\frac{E}{77}\right)} \text { for } 50<\mathrm{E}<200 \mathrm{MeV} \\
e^{\frac{-E}{250}} \text { for } \mathrm{E}>200 \mathrm{MeV}
\end{array}\right\}
$$

An earlier measurement had estimated the neutron flux at SUF as (1.07 $\left.{ }_{-0.30}^{+0.41}\right) \times 10^{-6}$ neutrons $/ \mathrm{cm}^{2} / \mathrm{s}$ in the $11.5-50 \mathrm{MeV}$ range[6]. However, this flux included both the high-energy hadronic component and the low-energy contribution from muon capture and secondaries from electromagnetic showers. For the measured flux, it was estimated that $62 \%$ of the contribution came from the high-energy component[6].

Neutrons arising from hadronic cascades can have energies greater than 50 $\mathrm{MeV}$ so only about $30 \%$ of these neutrons may lie in the 11.5 - $50 \mathrm{MeV}$ energy range. Additional uncertainty arises from the fact that a certain fraction of these neutrons $(\sim 40 \%)$ trigger the veto. Charged particles in the hadronic showers associated with the neutron production can also trigger the veto, causing the data analysis to reject such events. There is thus a factor of 3 uncertainty associated with the measurement of the neutron flux at this depth, based on the Run 19 analysis by Thushara Perera [7].

Based on the flux measurements, the rate of neutron production is taken as $(4.14 \pm 0.3)$ neutrons $/ \mathrm{kg} /$ day of $\operatorname{rock}[7]$.

Neutrons with the given production rate and spectra were allowed to propagate through 5 meters of rock and the emergent spectrum and flux used as the incident flux and spectrum on the detecting apparatus. Based on the normalization cited above, the external neutron flux incident on the shield was given by $(2.14 \pm$ $0.02) \times 10^{-6}$ neutrons $/ \mathrm{cm}^{2} / \mathrm{s}$. We define a $220 \times 220 \times 220 \mathrm{~cm}$ cube surrounding the shield and allow neutrons with the rate and flux specified to be thrown from the surface. The source rate incident on the shield is 0.621 neutrons/s. 


\subsubsection{Using the Run 19 results to normalize the external neutron flux}

Given the uncertainties associated with the incident external neutron flux, it was decided to use the fact that an earlier data run at the same site, Run 19, had arrived at a set of candidate events that was consistent with all events seen being neutrons[8][9]. The results of the earlier run were used to normalize the simulations of the external neutron background. The procedure adopted is given below.

The previous WIMP-search run, Run 19, employed four Berkeley Large Ionization and Phonon mediated (BLIP) detectors to search for dark matter. Each detector consisted of a $165 \mathrm{~g}$ disk consisting of a germanium crystal with neutron transmutation doped thermistors. A particle interaction gave rise to a temperature change which was recorded by the thermistors for the phonon measurement. The charge was measured using electrodes deposited on the top and bottom surface of each detector.

The data run recorded 27 nuclear-recoil neutrons candidates after all cuts had been applied for an exposure of $15.8 \mathrm{~kg}$-days[8]. This included 23 single-scatter events in the germanium detectors and 4 multiple-scatter events.

Based on the predictions of the ratio of germanium single-scatters for each multiple-scatter event from a Monte Carlo simulation of the external neutron background[7], all the 23 single-scatters were consistent with a neutron background. The expected rate due to the limiting neutron background was also consistent with the recorded data event rate of 1.71 events $/ \mathrm{kg}$-days[8].

The GEANT3 libraries used for the simulations were subsequently updated to include the angular dependence of the cross-sections of germanium [10]. The external neutron background simulations using the Run 19 geometry were re-run with the addition of the new cross-section libraries for germanium. This had the

effect of making the recoil spectra in the detectors softer at higher energies, reducing the event rate in the detectors between 10-100 keV. 
The simulations with the angular dependence of the cross-sections folded in predicted 43.36 single-scatter events and 3.86 multiples[11], giving an event rate of 2.99 events $/ \mathrm{kg}$-day for the $15.8 \mathrm{~kg}$-days exposure. From the data values, the event rate was determined as 1.71 events/kg-days. We estimated the neutron flux incident on the shielding assembly necessary to achieve the event rate as seen by the data in the Run 19 Monte Carlo simulations, i.e., a re-normalization factor of $\frac{1.71}{2.99}$ was applied to the estimate the incident flux. This was based on the assumption that all events seen in Run 19 were neutrons and that there was negligible leakage of low-yield surface electrons into the WIMP candidate data set. This was used as the input flux for the simulations with the Run 21 geometry.

\subsubsection{Addition of an inner polyethylene layer}

The limiting neutron background seen in Run19 led to an optimization of the shield geometry for the SUF experimental set-up. It was decided to add an additional layer of polyethylene within the inner lead inside the icebox cans to suppress this background. Holes cut into the poly allowed the placement of the tower with its complement of six detectors.

Monte Carlo simulations done prior to the installation had predicted a $(2.3 \pm 0.1)$ reduction in the neutron event rate due to the additional polyethylene[7]. Comparisons of simulations with the Run19 and Run21 detector and shielding assembly affirmed this result. Simulations run with and without the inner polyethylene layer for the Run 21 geometry indicated that the event rate fell from 2.9 events $/ \mathrm{kg} /$ days to 1.28 events/kg-days after adding the polyethylene. The factor of 2.26 decrease was consistent with the earlier simulations done at the time of the design of the experiment and indicated that the inner polyethylene, the one major difference in the shielding configuration between the two runs at the shallow site was instrumental in further suppressing the limiting neutron background.

According to the simulations, the single-scatter event rate in the germanium detectors fell from 2.71 events $/ \mathrm{kg} /$ day to 1.28 events $/ \mathrm{kg} /$ day in Run 21, a decrease 
of 2.21. Run 19 saw 23 germanium single-scatter events in 15.8 kg-days, giving an event rate of 1.45 events $/ \mathrm{kg} /$ day. The Run 21 data recorded 20 single-scatter events in 28.3 days for an event rate of 0.71 events $/ \mathrm{kg} /$ day. Based on the data, the event rate fell by 2.06 from Run 19 to Run 21 .

Both data and Monte Carlo are in agreement with the expected fall in rate due to the addition of the inner polyethylene. It must be noted, however, that the two runs had two different types of detectors and, while Run 19 had a $10 \mathrm{keV}$ energy threshold, Run 21 saw the threshold fall to $5 \mathrm{keV}$. This decrease in the threshold for the Run 21 data results in an efficiency normalized increase in the neutron recoil spectrum, leaving the change in the number of neutron events consistent with the increased shielding.

\subsection{Veto Anti-coincident Data at 3V charge bias}

After applying the data cuts as specified in Chapter III, data taken at 3V charge bias was reduced to a subset of veto anti-coincident nuclear-recoil events as shown in Figure 6.1. All cuts including the fiducial-volume and rise time cuts have been applied. A total of 20 single-scatter nuclear-recoil events were recorded in the four germanium detectors. These constitute the WIMP candidate data set. A phonon energy threshold of $5 \mathrm{keV}$ was imposed on all detectors except for Z1 whose larger noise required a $20 \mathrm{keV}$ threshold for better discrimination. In the case of the silicon detectors, only Z4 was considered as Z6 had a history of ${ }^{14} \mathrm{C}$ contamination of the detector.

\subsubsection{Efficiency of the fiducial-volume cut}

The neutron simulations provide a large sample of nuclear-recoil events which can be used to estimate the efficiencies of the various cuts applied to the data. The

energy dependence of the efficiencies of the data cuts may also be studied using 

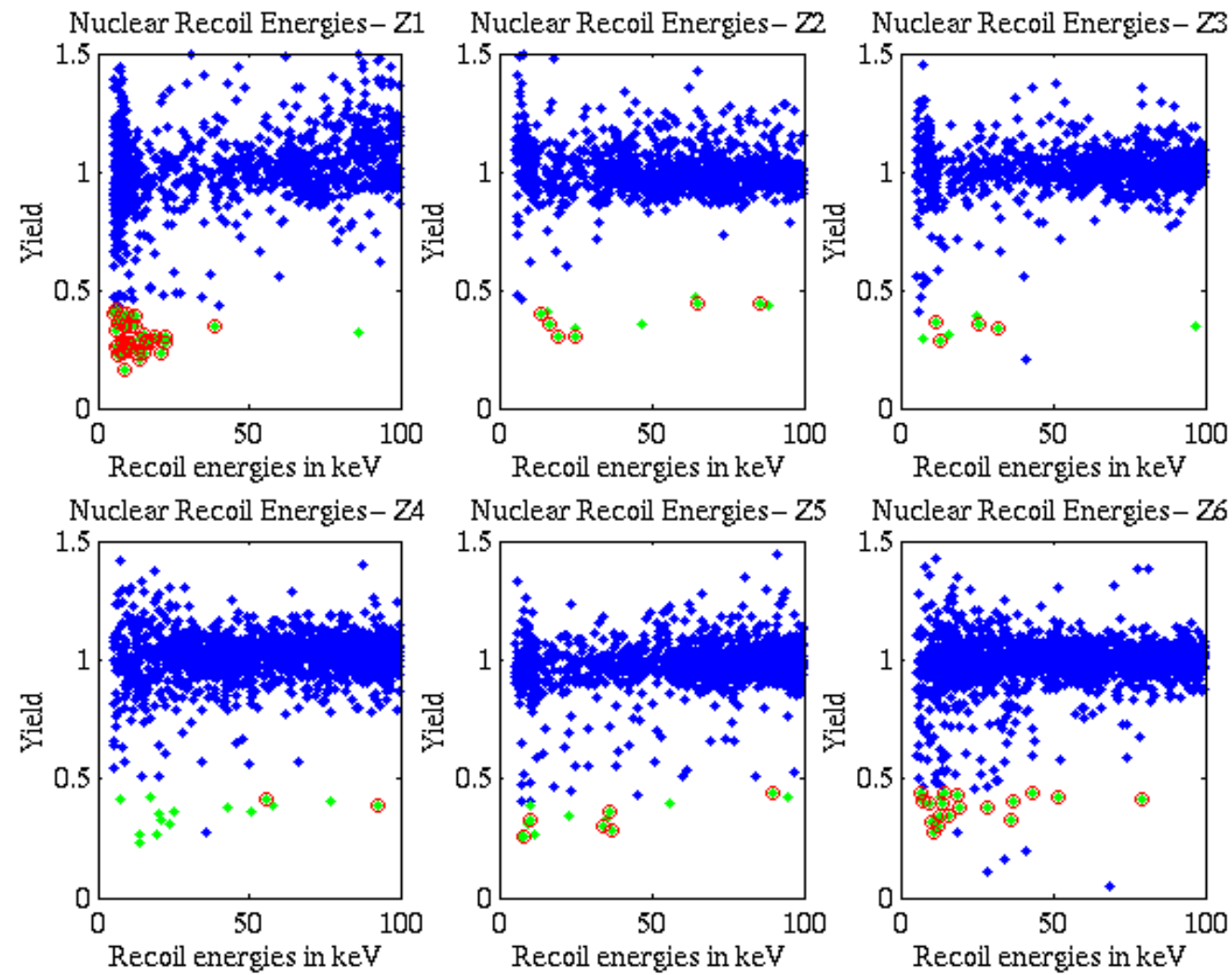

Figure 6.1: Veto Anti-coincident Data taken at 3V charge bias. Events in the signal region are shown in green. Single scatter nuclear recoil events are shown as red circles. As indicated, 20 single scatter events were seen in the four germanium detectors. The detectors are labelled from top left to bottom right where Z4 and Z6 are silicon detectors and Z1, Z2, Z3 and Z5 are germanium detectors. Detector Z1 had a higher energy threshold of $20 \mathrm{keV}$ while the other three germanium detectors had thresholds of $5 \mathrm{keV}$. The events in $\mathrm{Z} 6$ were not considered as Z6 was contaminated with ${ }^{14} \mathrm{C}$. 
the Monte Carlo simulations. Chapter V showed how, given the recoil energies, the charge energies and yield information may be extracted from the Monte Carlo. We examine the variation of cut efficiencies over the $5-100 \mathrm{keV}$ range using the Monte Carlo simulations.

As charge yield is poor for events that occur in the outer electrode of the detector, electron-recoil events that occur in this part of the device may suffer from poor charge collection and be mistaken for nuclear-recoil events. It is for this reason that only events which took place within the inner region of the detector are included in the data analysis. The neutron calibration data and the simulations of the calibration runs are used to estimate the efficiency of the fiducial-volume cut.

For the veto anti-coincident data set we define efficiency of the cut as the ratio of events passing the cut to all events examined. Figure 6.2 shows comparison of the energy variation of the cut efficiency for both data and simulations. For the data, the efficiency of the cut is calculated from the neutron calibration data. As the mean free path of neutrons in both silicon and germanium is of the order of a few centimeters, this ensures that the detector is uniformly illuminated by the source. This is necessary given our understanding that the WIMP flux is incident uniformly on the detecting apparatus.

The fall in efficiency between $5-20 \mathrm{keV}$ in detector $\mathrm{Z} 1$ is because the ionization noise becomes more important in determining the partition for the lower energies[2]. This detector also suffered from a large variation in the phonon signal due to a large gradient in the transition temperatures of its sensing elements. As both data and Monte Carlo indicate, the efficiency of the cut tends towards $85 \%$ at higher energies, reflecting the geometry of the detector where the inner electrode occupies $85 \%$ of the total volume. Figure 6.2 shows the variation of the efficiency of the fiducial-volume cut across the 10-100 keV energy range for both data and simulations. As the Monte Carlo output indicates, at high energies the efficiency tends towards $85 \%$, the geometrical area of the inner electrode. 

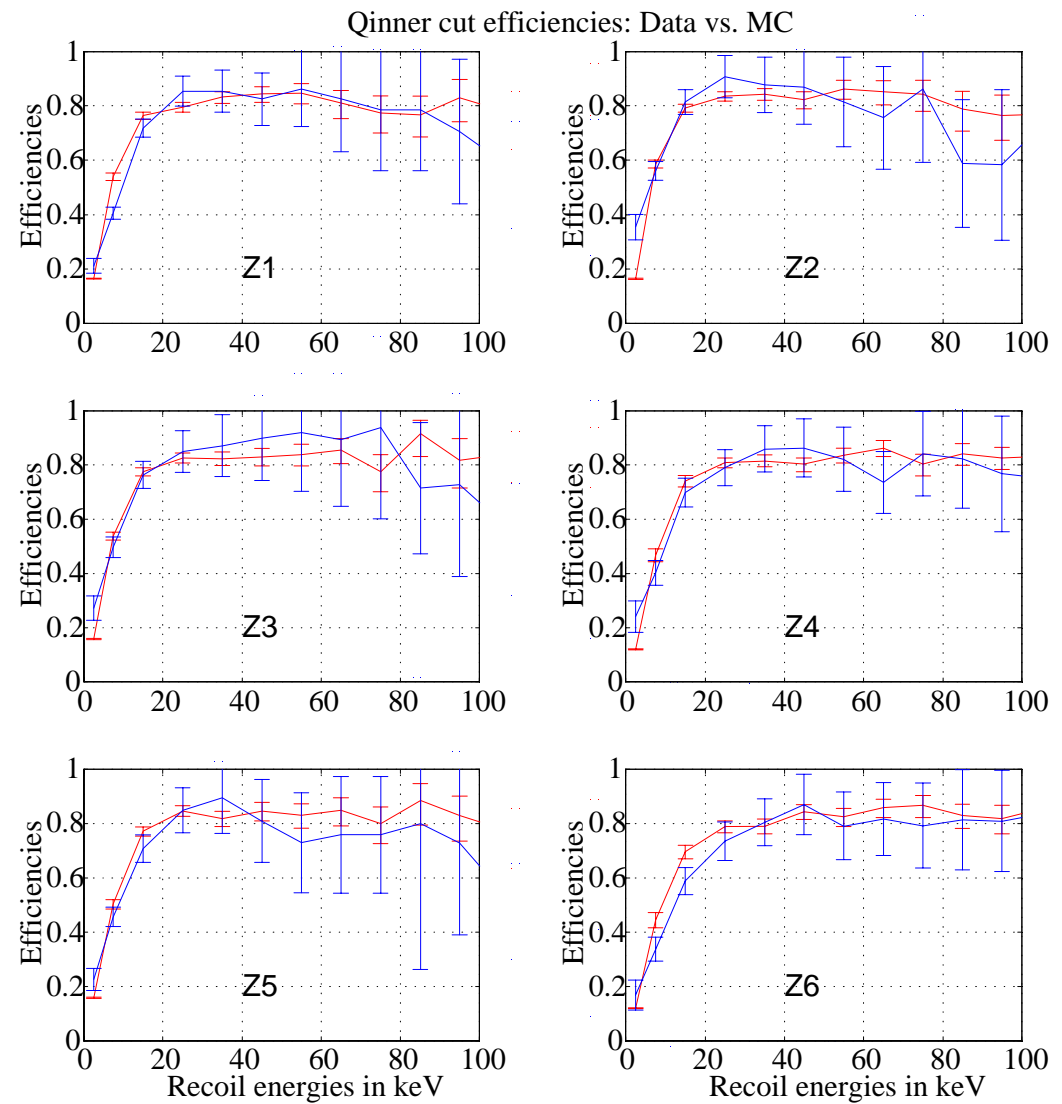

Figure 6.2: Energy dependence of the efficiency of the fiducial volume cut. The cut selects events which lie in $0.8<$ qpart $<1.2$ as explained in the text. The efficiency is given by the ratio of events passing the cut to all events. Cleaning cut have been applied to the data to select neutron candidates. The red curve is the simulations while the blue is the data. 


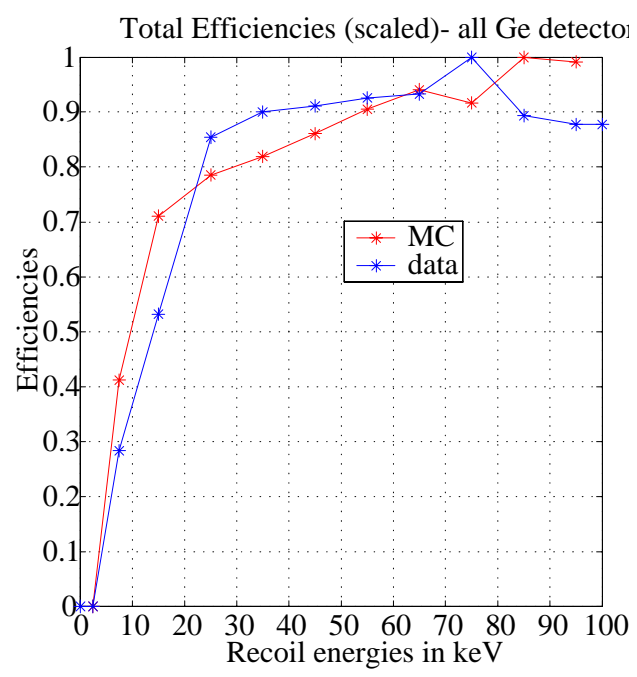

Figure 6.3: Total relative efficiency for single scatter events in the four germanium detectors for both data and simulated output.

\subsubsection{Total efficiency}

The nuclear-recoil band cut selects events within $\pm 2 \sigma$ of the nuclear-recoil band mean and thus has a $95 \%$ efficiency across the energy range of interest. The rise time cut is energy dependent rising from $10-15 \%$ at $5 \mathrm{keV}$ to $40-45 \%$ at 10 $\mathrm{keV}$ to $50-60 \%$ at higher energies[1][12]. The cut selecting events that are not in coincidence with a trigger in the veto cut is a flat $80 \%$ across the entire energy range given that the veto trigger rate is $5.6 \mathrm{kHz}$ and the dead time for each trigger is $40 \mu s[2]$.

Figure 6.3 shows the energy dependence of the total efficiency after all cuts for both data and simulations for the single-scatter events in the germanium detectors. The efficiencies have been scaled so that the maximum efficiency is 1 . Figure 6.4 shows a similar comparison for the silicon detectors. The resulting exposure of the Run is determined from the maximum value of the efficiency over the 5-100 keV range for the germanium detectors. 


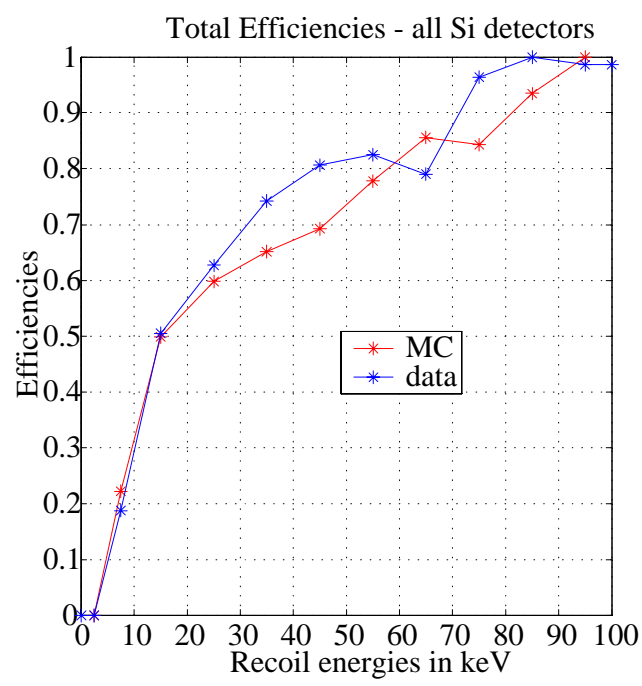

Figure 6.4: Total relative efficiency for single scatter events in the two silicon detectors for both data and simulated output.

\subsubsection{Results of the first analysis of the 3V WIMP-search data}

The first analysis of the WIMP-search data taken at 3V charge bias had a raw exposure of $66.6 \mathrm{~kg}$-days which reduced to $28.3 \mathrm{~kg}$-days after cuts for the four germanium detectors. The veto anti-coincident data set was reduced to 20 singlescatter events in the germanium detectors, 2 single-scatters in the silicon detector Z4 and 8 multiple-scatter events after the application of all cuts. While events in the silicon detector and multiple-scatters arise from neutrons, the 20 single-scatters in germanium may be neutrons or possible WIMPs, or could result from low-yield surface electrons leaking into the signal region.

Eight multiple-scatter events were seen in the first analysis of the Run 21 $3 \mathrm{~V}$ data. Events that produce multiple-scatters that fall in the signal region of the two (or more) detectors are clearly neutrons. Some of the single-scatters, thus, are also neutrons with the ratio of single-scatters to each multiple being provided by the 
simulations of the external neutron background. The next section outlines how the simulations helped arrive at the conclusion that all the germanium single-scatters seen in Run 21 were consistent with a neutron background.

\subsection{Consistency of the Neutron hypothesis}

\subsubsection{V WIMP-search data}

Run 21 saw twenty single-scatters in germanium, two singles in silicon and eight multiple-scatter events in the veto anti-coincident WIMP-search data. Of the multiples, there were two triple scatter events, one double-scatter between two non-nearest-neighbor detectors and five double-scatters between nearest-neighbor detectors. While the triples and non-nearest-neighbor doubles were clear nuclearrecoil events with yield plots indicating a clean separation between electron-recoil and nuclear-recoil events, the possibility of significant contamination by low-yield surface electrons in the nearest-neighbor doubles, as seen in Figure 6.5 did not allow for a similar conclusion about this population. As we were unable to quantify the leakage of low-yield betas resulting from surface contamination or ejectrons into this subset of multiple-scatter events, is was decided to exclude these events from the dark matter analysis to set limits on the WIMP-nucleon cross section.

\subsubsection{Predictions of the external neutron simulations}

After applying the efficiencies of the data cuts for the given run exposure, the simulations predicted 13.3 single-scatter events in the germanium detectors, 2.7 single-scatters in Z4 and 5.6 events which scatter in more than one detector based on the 66.6 raw live days of data. Here all detectors had a $5 \mathrm{keV}$ phonon energy threshold except Z1 which had a $20 \mathrm{keV}$ threshold. The multiples consisted of 0.5 triples, 2 non nearest-neighbor doubles and 3.1 nearest-neighbor doubles. 


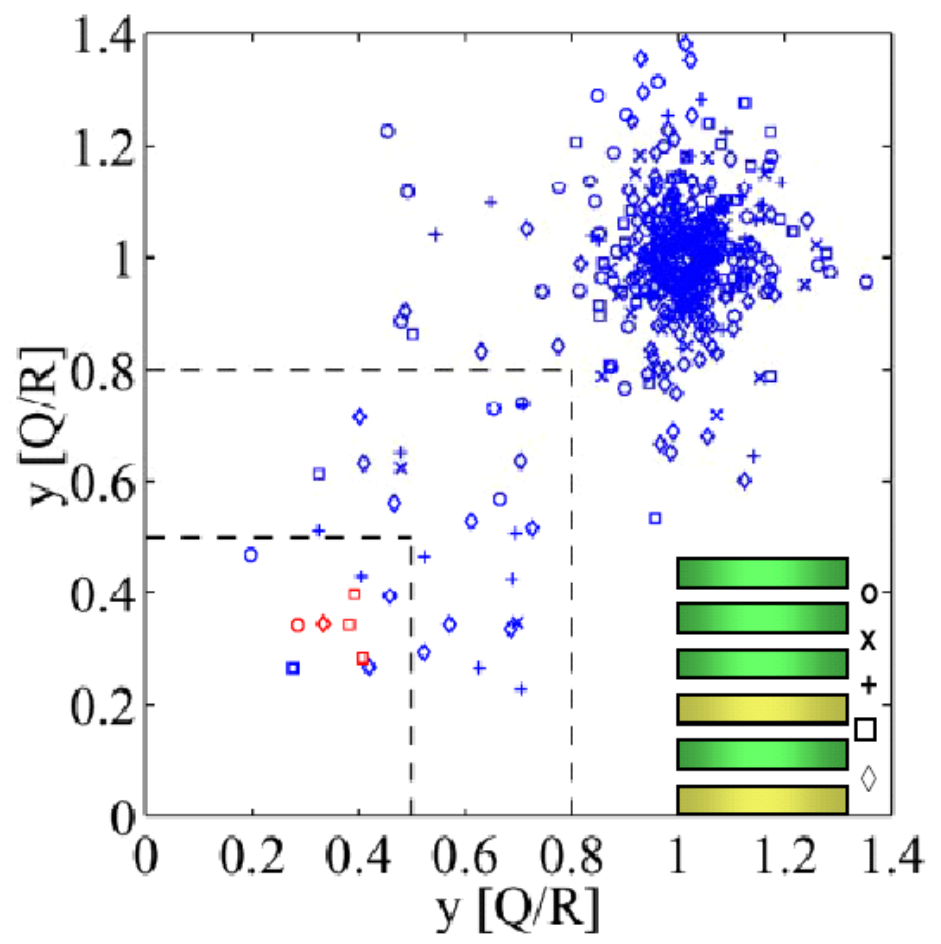

Figure 6.5: Yield vs. yield plots for the population of double scatter events between nearest neighbor detectors in the WIMP search veto anti-coincident data set. As may be seen, there is poor separation between events in the signal and background bands. The cartoon of the detector stack shows the detectors in which these scatters took place (taken from [14]). 


\begin{tabular}{|c|c|c|}
\hline Events & Expected number of neutrons & Data \\
\hline Total singles & $(16.0 \pm 0.2)$ & 22 \\
\hline Single scatters in Ge & $(13.3 \pm 0.2)$ & 20 \\
\hline Single scatters in $\mathrm{Si}$ & $(2.70 \pm 0.08)$ & 2 \\
\hline Nearest neighbor doubles & $(3.10 \pm 0.08)$ & 5 \\
\hline Non-nearest neighbor doubles & $(2.0 \pm 0.1)$ & 1 \\
\hline Triples & $(0.50 \pm 0.03)$ & 2 \\
\hline
\end{tabular}

Table 6.1: Expected Number of Neutrons for the 3V Veto Coincident Data for data and simulations. Event rates give ratio of events passing all cuts to raw livedays of the simulations. The expected number is for 66.6 live days.

Table 6.1 shows the comparison of events seen in the detectors to the predictions of the simulations of the external neutron background. The simulations are normalized from the Run 19 results to factor out the uncertainty in the production rate. Event rates are expressed in raw live days and expected numbers determined for 66.6 live days.

For the dark matter analysis, the simulations are used to predict the relative rates of single to multiple-scatter events and the relative rates of scatters in the silicon and germanium detectors so as to make the predictions normalization independent. The silicon singles and multiples provide a measure of the neutron background. Based on the simulations, the ratio of germanium singles to a multiplescatter was 2.29 .

However, the presence of contamination in the population of nearest-neighbor double-scatters and the inability to correct for false events due to double-scattering of surface electrons made it necessary to exclude this data set from the WIMP limits analysis. The number of germanium singles to each multiple (triples and non-nearest-neighbor doubles) alone was predicted to be 5.32 by the simulations. Coupled with the prediction of a leakage of $(1.2 \pm 0.3)$ surface events into the signal region for the germanium detectors and $(0.8 \pm 0.6)$ events for the silicon scatters[1], 
this suggested that all the WIMP candidate events seen resulted from backgrounds. As noted previously, the phonon energy threshold considered was $20 \mathrm{keV}$ for Z1 and $5 \mathrm{keV}$ for the other detectors. Only $\mathrm{Z} 4$ was considered for the silicon detectors with Z6 being excluded due to the ${ }^{14} \mathrm{C}$ contamination on the detector surface.

\subsubsection{Testing the neutron hypothesis}

The 20 single-scatters seen in the Run 21 were consistent with a neutron background, confirming the hypothesis that the sensitivity to WIMP detection at SUF was limited by the existence of an external neutron background. A comparison of the recoil spectra for the single-scatter nuclear-recoil events in the WIMP-search data shows good agreement with the spectrum generated by the simulations of the external neutron background, as shown in Figure 6.7.

A maximum likelihood ratio test on the number of nuclear-recoil events seen in Runs 19 and 21 indicated that a single neutron background would result in a less likely combination of Ge Singles, Ge (triple and non-nearest-neighbor doubles) multiples and Si singles $36 \%$ of the time.

We can also use the Kolmgorov-Smirnov statistical test to determine if the veto anti-coincident nuclear-recoil events seen in the data were consistent with an external neutron background. By comparing the cumulative distribution function of the data with that of a hypothetical source producing the events, we can estimate the probability that a random set of events taken from this hypothetical distribution is in worse disagreement than the data. The D statistic, as shown in Figures 6.10 and 6.11 indicate the largest variation in the two spectra. The significance points to the agreement between the two spectra with $5 \%$ significance or higher indicating a high possibility of the two spectra arising from the same sources.

Figure 6.7 shows the results of the K-S test for the single-scatter nuclearrecoil events in the four germanium detectors indicates that the deviation between 


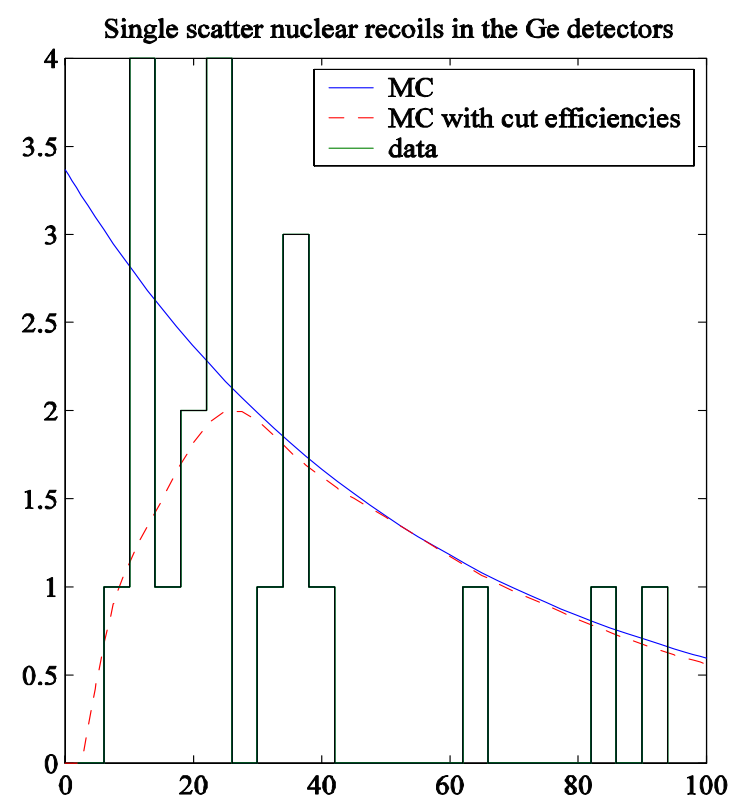

Figure 6.6: Comparison of the nuclear recoil spectra for the single scatter events in the Ge detectors with the spectrum of the external neutron background as generated from simulations. 


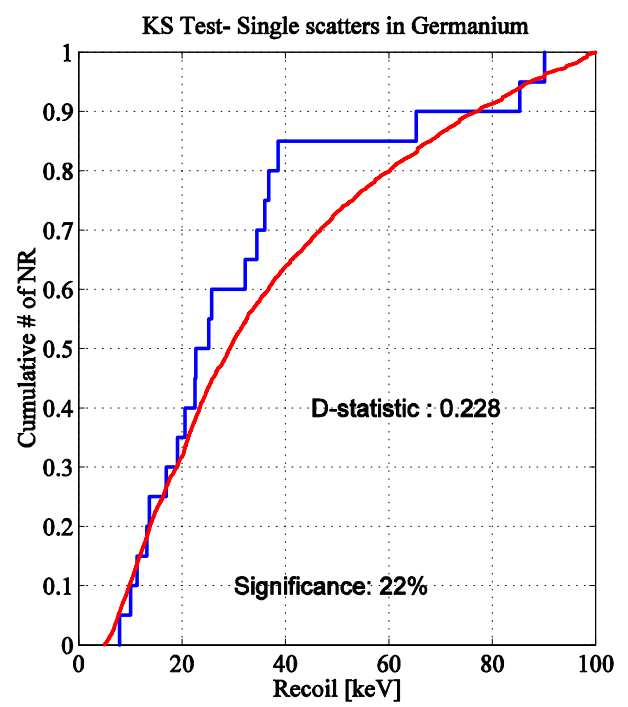

Figure 6.7: K-S test of the single scatter nuclear recoil events in the four Germanium detectors. The test compares the recoil spectra of the distribution generated by the external neutron background and the events seen in the veto anti-coincident data. As can be seen, the test shows a high value of significance between the histogram, which constitutes the data, and the smooth curve, which is the simulated output. This serves to confirm that the events seen in the WIMP search data are consistent with those produced by an external neutron background.

the observed and simulated nuclear-recoil spectral shapes would be larger $22 \%$ of the time. A similar K-S test for both the single and multiple-scatter nuclearrecoil events in the data set indicates that the deviation between the observed and simulated nuclear-recoil spectra would be larger $36 \%$ of the time.

Figure 6.8 shows a similar K-S test for the agreement between the singlescatter events seen in the germanium detectors and the single-scatter events in the simulations of the veto coincident and anti-coincident neutron background. The test of the observed nuclear-recoil spectrum in the data and the expected spectra from the simulations yields $23 \%$ for the externals and $68 \%$ for the internals, indicating good agreement between the shapes. 


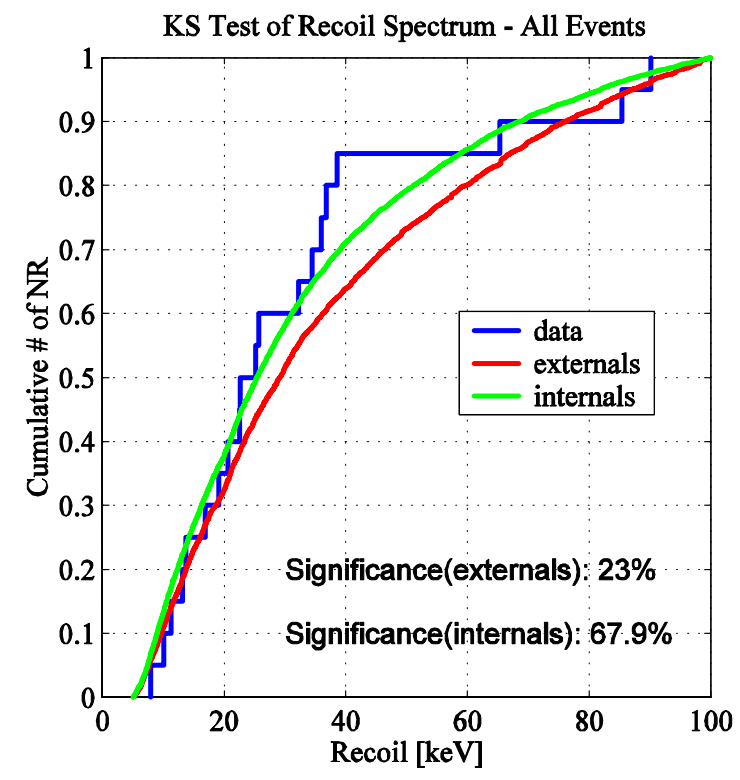

Figure 6.8: K-S test of the agreement between the observed recoil spectra of the single scatter nuclear recoil events seen in the veto anticoincident data set and the internal and external neutron simulations. 
The presence of a limiting background as indicated by the occurrence of neutron events in the WIMP candidate data necessitates a statistical subtraction of the neutron background to be able to compute the sensitivity to the detection of WIMP dark matter by the experimental data. The first analysis of the Run $213 \mathrm{~V}$ data was used to set new limits on the WIMP nucleon cross section as is explained in the next section.

\subsubsection{Improved Limits}

Figure 6.9 shows the limits on the spin independent WIMP nucleon elastic scattering cross section set by the first analysis of the experimental data of Run $21[1]$.

The plot shows the variation of the WIMP mass with the WIMP-nucleon cross section. Each point in the parameter space is representative of the possibility of WIMPs of a particular mass and interaction rate which may be explored by experimental observations. The shaded regions represent WIMP parameter space as predicted by theoretical models. Each curve represents an experimental probe with the area above any particular curve representing the excluded region of parameter space as evinced by the non-observation of WIMPs in an experimental data set at the $90 \%$ Confidence Level. Each curve thus shows the 90\% Confidence Level upper limits on the WIMP nucleon cross section for a range of WIMP masses from $10-500 \mathrm{GeV}$. The regions below the curve represent parameter space that becomes available for experimental probes as better background rejection techniques increase sensitivities to WIMP detection. The curves are normalized[13] using the Helm spin-independent form factor $\mathrm{A}^{2}$ scaling values for a characteristic WIMP velocity of $220 \mathrm{~km} / \mathrm{s}$. The mean velocity of the Earth is taken as $232 \mathrm{~km} / \mathrm{s}$ and a local halo density of $0.3 \mathrm{GeV} / \mathrm{c}^{2} \mathrm{~cm}^{-3}$.

The limits exclude new parameter space for WIMPs of masses 8 - 20 $\mathrm{GeV} / \mathrm{c}^{2}[3]$ and give limits on the spin-independent WIMP interactions that are 


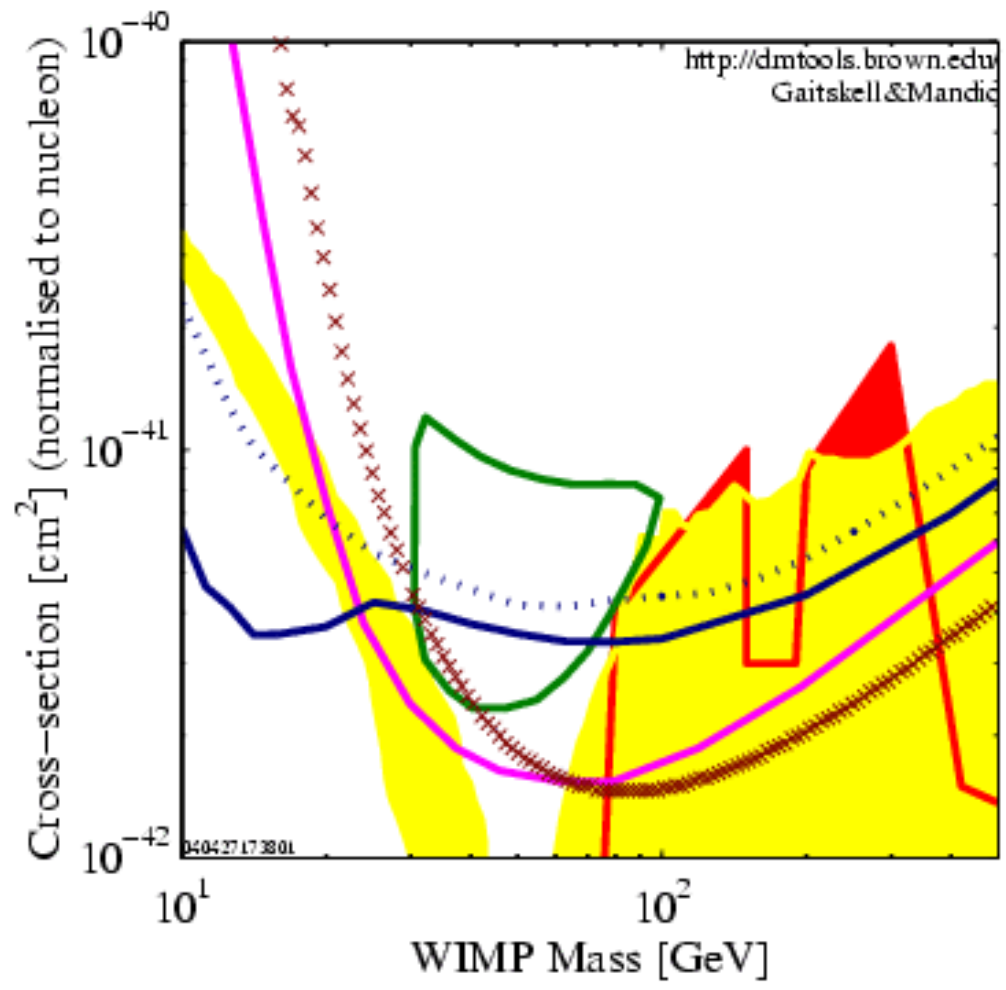

Figure 6.9: Exclusion Limits on the spin-independent WIMP-nucleon elastic scattering cross section for WIMP masses of $10-100 \mathrm{GeV}$. The solid blue line represents the limit set by the first analysis of the Run 21 data which includes the statistical subtraction of the neutron background. The brown crosses line are the limits set by Edelweiss while the magenta represent those set by ZEPLIN. The yellow and red shaded regions represent allowed regions of SUSY parameter space. The green closed circle represents the DAMA $3 \sigma$ allowed region. 
lower than previous limits set by the experiment at the same site for higher WIMP masses. The $5 \mathrm{keV}$ threshold of the data resulted in better limits for WIMPs of low masses. The dip seen in the limit curve arises from the fact that the many detected events seen between $30-40 \mathrm{keV}$, as seen in Figure 6.12, could not have been produced by interactions of low mass WIMPs.

The limits are incompatible with the model independent annual modulation signal of the DAMA dark matter experiment at $99.98 \%$ Confidence Level[1] provided we consider standard WIMP interaction and halo models.

\subsection{Using Maximum Likelihood Analysis Tech- niques for beta rejection}

The first analysis of the Run $213 \mathrm{~V}$ used a flat cut on the rise time of the phonon pulse to discriminate against low-yield surface electrons leaking into the signal region and being mistaken for nuclear-recoil events. The WIMP-search data analysis, however, indicated that there was significant beta contamination into the nuclear-recoil band, more specifically among the population of nearest-neighbor double-scatters. As it was necessary to better quantify the double-scattering of surface electrons in the nuclear-recoil band, it was decided to exclude this event population from the results of the first analysis of the Run 21 data.

The exclusion of the nearest-neighbor double-scatter events resulted in a fewer number of multiple-scatter events. The number of multiples and silicon singles are employed in estimating the neutron background and doing a statistical subtraction of the neutron contribution to the WIMP candidate data set. The small number of these events reduced the statistical power gained by making estimates of the neutron contribution to the single-scatter events in the germanium detectors[1].

The analysis of the combined $3 \mathrm{~V}+6 \mathrm{~V}$ data sought to improve on the number of multiples seen from a larger data set, and thus reduce the statistical error on 
the multiples which dominates the effectiveness of the neutron subtraction. The need for a proper estimate of the leakage of low-yield surface electrons into the nuclear-recoil band was the basis of defining an optimal cut to separate out the $\beta$ and neutron contribution in the signal region for the analysis of the combined $3 \mathrm{~V}$ $+6 \mathrm{~V}$ data from the run[3]. While the first analysis of the Run21 data constituted the thesis work of Tarek Saab, optimizing the discrimination against beta leakage into the signal region for the entire Run21 data was part of the dissertation of Don Driscoll. In both the cases, the data analysis had to be complemented by an analysis of the limiting neutron background as evinced by simulations. My work focussed on simulating the limiting neutron background seen by the experiment. The efficiencies of the data cuts were applied to the simulations and estimates made of the neutron contribution to the WIMP-search data based on the modelled output. The efficiencies of the data cuts were also tested against the simulations as they provide a large sample of nuclear-recoil events. The analysis was repeated for the analysis using the maximum likelihood distribution function as these cuts were optimized to characterize the beta discrimination in the WIMP-search data.

In Chapter V, we showed how the maximum likelihood ratio cuts were applied to the simulation of the neutron calibration. As described therein, the comparisons of rates and spectra of data and Monte Carlo indicated a slight overestimation in rates for the simulations. This was particularly evident in the case of the single-scatters and the nearest-neighbor doubles population. The slight disagreement in the recoil spectra for data and simulations in the case of the neutron calibration may be a consequence of poor statistics in the high-energy regions affecting the fitting functions generated for the likelihood analysis. The analysis had laid out a procedure to define maximum likelihood ratio parameters using the calibration data and define fitting functions that give the likelihood of an event being a neutron or a beta. These fitting functions would then be applied to a population of events in the WIMP-search data to arrive at an estimate of the beta leakage into the signal region. Statistical limitations in the defining of these parameters could affect the nature of our ability to assess the efficiency of the cuts. In such a case, 
we make a conservative estimate of the efficiencies as applied to the simulations to extract the neutron subtraction parameters necessary for the dark matter analysis.

\subsubsection{Estimating the efficiencies of the data cuts}

As similar procedures of applying the efficiencies of the data cuts are followed for the external neutron simulation which provides the ratios necessary for the neutron subtraction, it is necessary to characterize the difference in the rates for these two event populations and arrive at a conservative means of estimating efficiencies and exposure for the WIMP-search data.

\section{Calibration Neutrons at $3 \mathrm{~V}$ charge bias}

We begin by quantifying the variation for the $3 \mathrm{~V}$ neutron calibrations. The efficiencies cuts were applied to the simulations of the $3 \mathrm{~V}$ calibration and the spectral distribution of the single-scatter nuclear-recoil events passing all cuts was obtained. A similar distribution was obtained from the data. Figure 6.10 shows the comparison of spectra for $3 \mathrm{~V}$ single-scatter nuclear-recoil events in germanium for data and Monte Carlo output. A Kolmgorov-Smirnov test to check the agreement between the two data sets indicated a significance of $<1 \%$. This does not indicate a good agreement between the two recoil spectra and suggests that limited statistics while defining the fitting functions may be the reason for this discrepancy. The differential distribution of the nuclear-recoil spectra over $10-100 \mathrm{keV}$ suggest an overestimate in the efficiencies for the modelled output. This is accounted for in the analysis of the external neutron simulation by making a conservative estimate of the efficiencies as specified in Section 6.5.

To estimate the efficiencies, the events in the signal region were pre-selected using the fiducial-volume and the nuclear-recoil band cuts. We choose energy bins with edges given by [5 1020304050607080 100] where the energies are in keV. 


\section{KS Test of Recoil Spectrum}

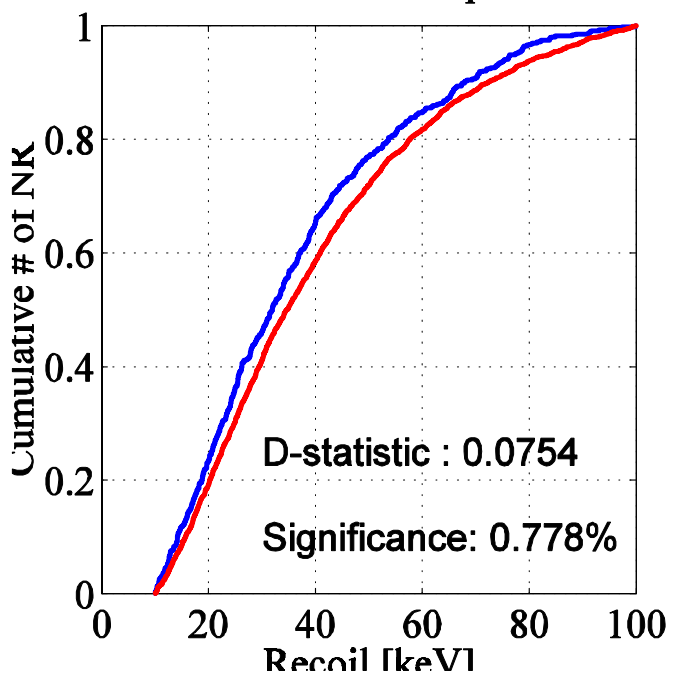

Figure 6.10: KS test of the single scatter nuclear recoil energy distribution for the case of the $3 \mathrm{~V}$ calibration data and simulated output using the likelihood ratio analysis. There is not very good agreement between data and simulations which could arise due to statistical limitations while defining the fitting functions for the cuts. The recoil spectra represent single scatter events in the three germanium detectors Z2, Z3 and Z5. 
The ratio of events passing all cuts including the single-scatter and the maximum likelihood cuts for singles to those passing the pre-selected events is used to estimate the efficiency of the likelihood function ratio cuts. For the Monte Carlo distributions, the ionization energies are modelled as specified in Chapter $\mathrm{V}$ and events are pre-selected based on occurrence in the inner electrode and within the 5 -100 $\mathrm{keV}$ energy range. After selecting single-scatter events and applying the likelihood function ratio for the single-scatters, we are able to estimate efficiencies using the simulated distribution.

Figure 6.11 shows the energy distribution of the efficiencies for both data and simulations for the single-scatter events in the three germanium detectors. It is apparent that the efficiencies for the data output are consistently lower than the simulations over the energy range of $10-100 \mathrm{keV}$. The black solid line shows a fit to the data efficiencies. The fall in efficiency above $70 \mathrm{keV}$ is due to poor statistics in events seen in the data. Again, this discrepancy is accounted for by making a conservative estimate of the efficiencies over the energy bins for both the calibration and the veto coincident data.

\section{Veto Coincident Neutrons at $3 \mathrm{~V}$ charge bias}

The large veto coincident neutron population seen in Run 21 provides another means of determining agreement between data and simulations. Chapter $\mathrm{V}$ described a similar comparison of data and simulations for the various muoncoincident nuclear-recoil event populations. We perform a K-S test to see if the single-scatter nuclear-recoil distributions seen in both cases are significantly different from each other. As indicated in Figure 6.12, there is good agreement with the significance $=18.1 \%$. We then estimate the efficiencies of passing the likelihood ratio cuts for the two cases in a manner similar to the calibration analysis above. The efficiency distribution as a function of energy in the $10-100 \mathrm{keV}$ scale is shown and the variation between the data and simulations values is recorded. As is indicated in the efficiency plot in Figure 6.13, the data efficiencies are consistently 


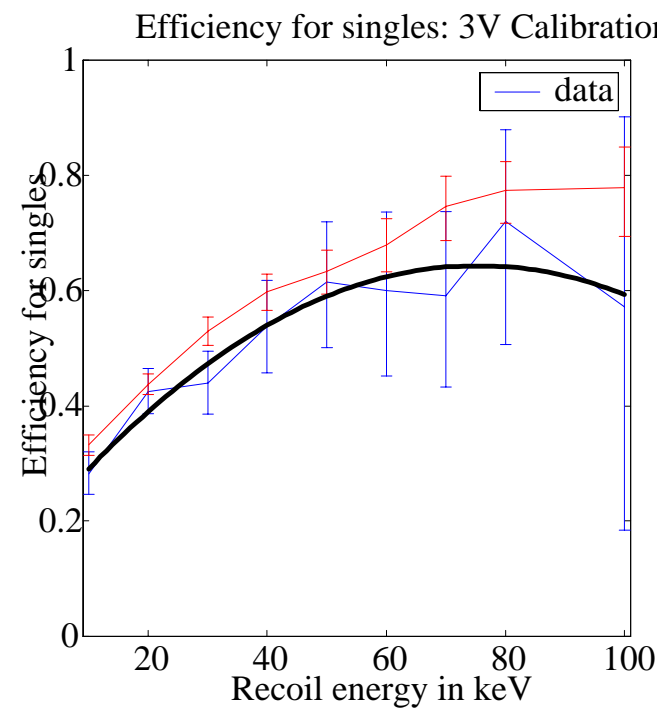

Figure 6.11: Varation of cut efficiencies with energy for both data and simulations. There is a slight overestimate in the efficiencies of the Monte Carlo simulations. The efficiencies are considered for single scatter events in the three germanium detectors Z2, Z3 and Z5. 


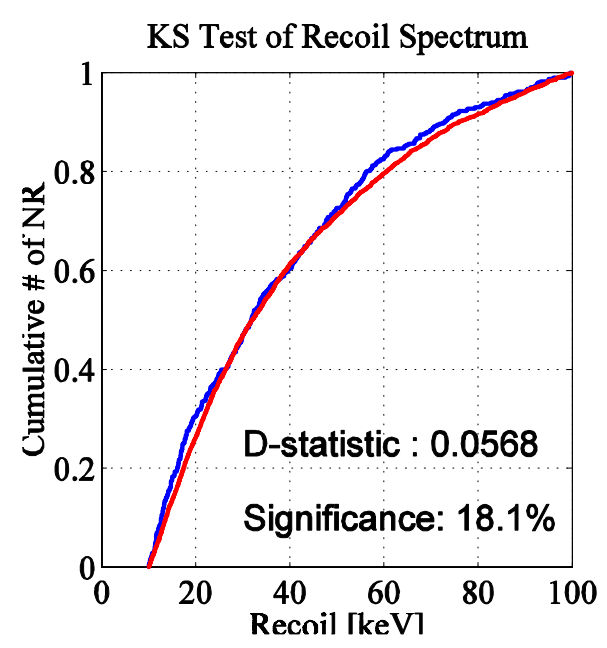

Figure 6.12: K-S test of the single scatter nuclear recoil energy spectrum for the case of $3 \mathrm{~V}$ veto coincident neutrons. The test compares the distribution of data and simulated output. The recoil spectra represent single scatter events in the three germanium detectors Z2, Z3 and Z5.

higher than those modelled for the Monte Carlo distributions over the entire 10 $100 \mathrm{keV}$ energy range. We make a conservative estimate of the efficiency over each energy bin by considering the data values for both the calibration and the internal neutron cases. The fitting function arrived at is then used applied to the veto anti-coincident nuclear-recoil single-scatter events as explained in the next sections.

\section{Neutron calibration at $6 \mathrm{~V}$ charge bias}

A similar analysis was undertaken for the calibration and veto-coincident data taken at $6 \mathrm{~V}$ ionization voltage. For the calibration data set at $6 \mathrm{~V}$ bias, the K-S test indicated a significance of $<1 \%$ indicative of the fact that there is a slight disagreement between data and simulations. The efficiencies were studied over the 10-100 keV recoil energy range for both data and simulations. The dip in the efficiency curve above $80 \mathrm{keV}$ is related to poor statistics in the data. 


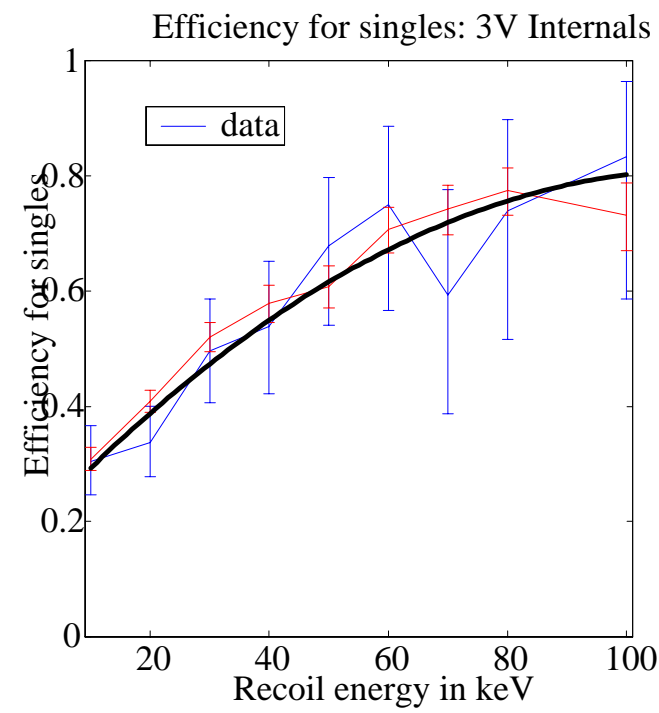

Figure 6.13: Variation of the cut efficiencies as applied to data and Monte Carlo as a function of energy. The data efficiencies are consistently lower than the simulations over the entire energy range. The efficiencies are considered for single scatter events in the detectors Z2, Z3 and Z5. 


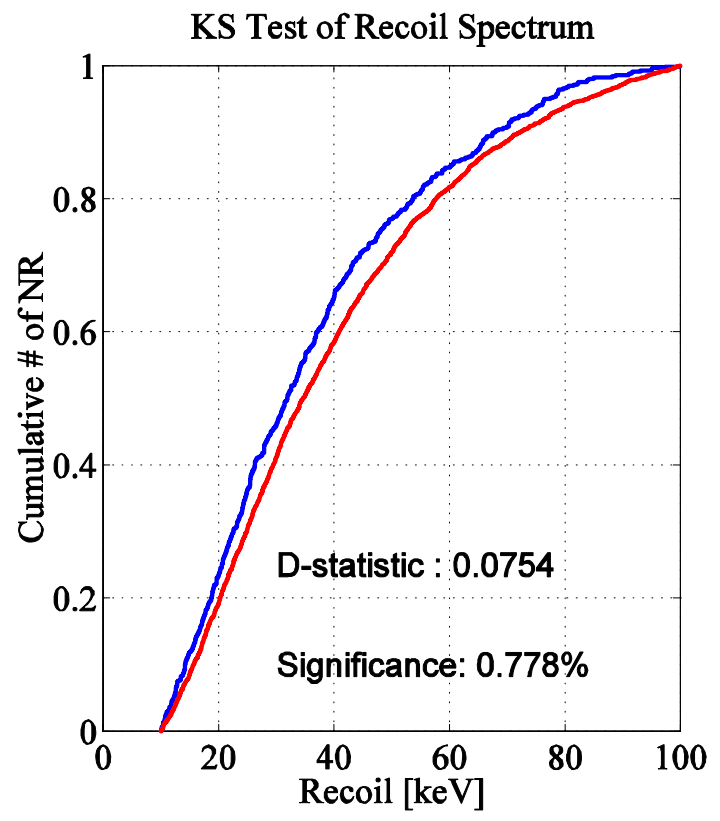

Figure 6.14: K-S test for the single scatter nuclear recoil candidates for the $6 \mathrm{~V}$ Neutron calibrations. As indicated, there is not very good agreement between the two spectral distributions. The recoil spectra are for single scatters in the three germanium detectors used for the analysis. 


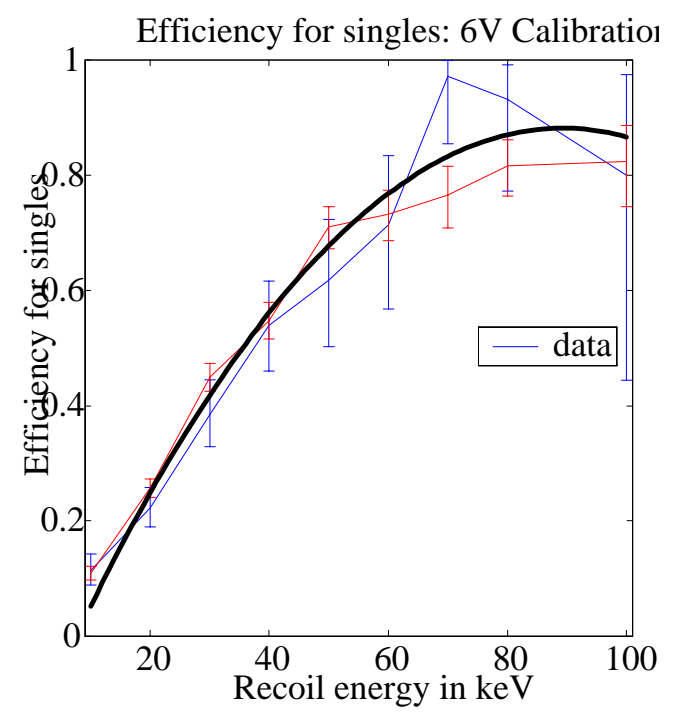

Figure 6.15: Variation of efficiency over $10-100 \mathrm{keV}$ for both data and simulations. The drop in effiiciency above $80 \mathrm{keV}$ comes from poor statistics in the data. The efficiencies are plotted for Z2, Z3 and Z5.

\section{Veto coincident neutrons at $6 \mathrm{~V}$ charge bias}

The muon-coincident neutron data set was examined for agreement in the single-scatter nuclear-recoil energy spectra for both data and simulations. The efficiency variation is as shown in Figure 6.17. A fit is made to the efficiency over the $10-100 \mathrm{keV}$ range as is indicated in the representation. As in the case of the $3 \mathrm{~V}$, we make a conservative estimate of the efficiencies over each energy bin by considering the values for both the calibration and internal neutron data sets. The data efficiencies and the fit to the values are chosen for this estimate as they are lower than the Monte Carlo values for the energy distribution.

These are the cut efficiencies for the data taken at $6 \mathrm{~V}$ charge bias. These efficiencies are then applied to the simulation of the veto anti-coincident neutrons to estimate the rates and expected number of neutron candidates after all efficiency cuts. The procedure is outlined in the next section. 


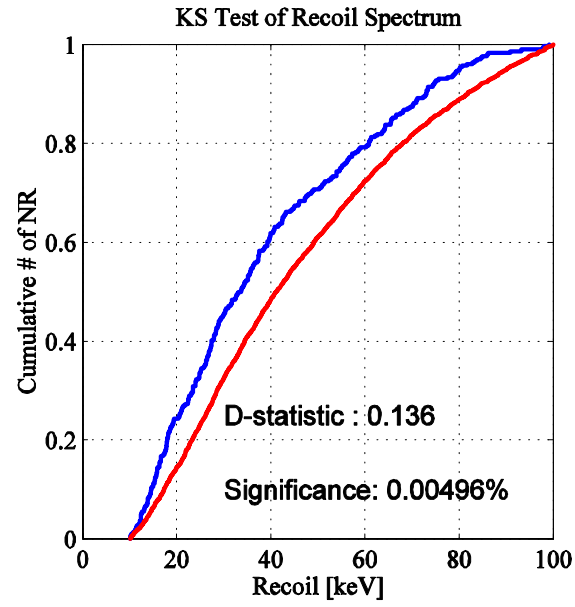

Figure 6.16: K-S test for the veto coincident single scatter nuclear recoil candidates at $6 \mathrm{~V}$ charge bias. The figure shows the comparison of recoil spectra for the data and simulations. The spectra are for Z2, Z3 and Z5.

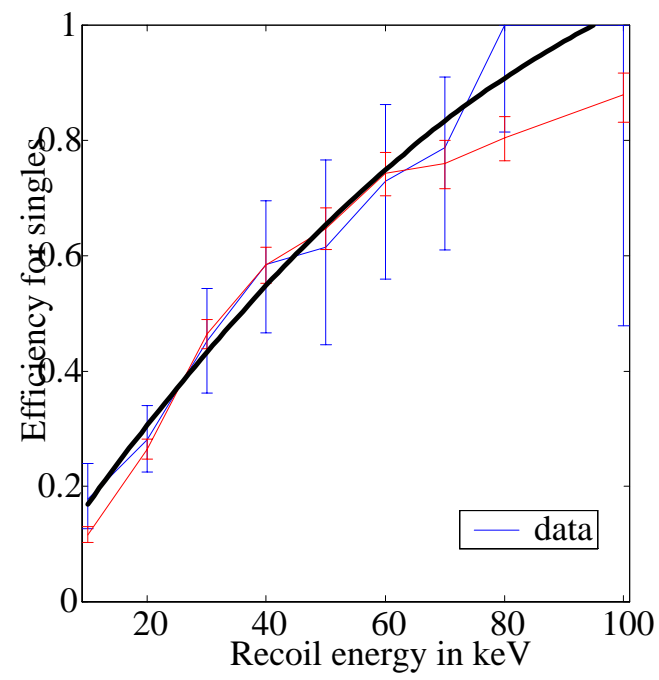

Figure 6.17: Variation of efficiency for the 6V veto-coincident neutrons between 10-100 keV for both data and modelled output. The data efficiencies are lower than the Monte Carlo over the energy range and the detectors considered are Z2, Z3 and Z5. 


\subsubsection{Estimating the neutron background}

The neutron subtraction method relies on applying the efficiencies of the data cuts to the external neutron simulations to make estimates on the limiting neutron background. The slight discrepancy between data and simulations for the two test cases of the neutron calibration and the veto-coincident neutrons is accounted by making a conservative estimate of the efficiencies applied to the external neutron simulations. Over the given energy bins, we chose the most conservative value of total efficiency from the case of the calibration and internal neutrons. The cut efficiencies were applied individually to the simulations of the external neutrons for both the $3 \mathrm{~V}$ and $6 \mathrm{~V}$ charge biases.

\subsubsection{Limits on the WIMP-nucleon cross section}

After applying the efficiencies of the data cuts at $3 \mathrm{~V}$ and $6 \mathrm{~V}$ bias to the simulations of the external neutron background, we arrive at a prediction of the estimated neutron contribution to the WIMP-candidate data set. The ratio of single-scatter events in the three germanium detectors to the multiple-scatter events provided by the simulations was used in the neutron subtraction for calculating the limits on the WIMP-nucleon cross section set by this data set.

The likelihood ratio analysis method sought to determine a better way of quantifying the beta leakage into the signal region and evolve a means of better discriminating between the bona-fide nuclear-recoil events and the beta-induced population in the WIMP-search data. The use of the neutron simulations lay in providing a sample population of nuclear-recoil events on which to test the effectiveness of the cuts. The cuts were tested on the simulations of the neutron calibration and the veto coincident neutron population for the $3 \mathrm{~V}$ and $6 \mathrm{~V}$ data. Based on the results of the cross checks and analysis shown in the Sections 6.4.1, we understand the limitations of this method.

Based on the maximum likelihood ratio analysis and on applying the effi- 
ciencies of the cuts formulated from this technique to the simulations of the external neutron background, we set new limits on the WIMP-nucleon cross section for WIMP masses between 10-100GeV. Given below are limits on the WIMP-nucleon cross section set by the extended analysis of the Run 21 data. The fit of the energy spectrum of the 19 single-scatter nuclear-recoil events seen in the data to the spectrum of the external neutron background is not very good. This suggesting either the possibility of leakage of another source of backgrounds into the WIMPcandidate data set or that we do not discount the possibility of detecting low mass WIMPs. We immediately rule out the second option, on account of the fact that a more sensitive measurement, described in Chapters VII and VIII, rule out the presence of WIMPs in the same parameter space. This analysis, therefore, explores possible limitations of the method which may have arisen due to statistical limitations in the defining of these parameters.

To try and understand the disagreement in the spectra, we examine the a-priori assumptions made in the calculations. We make the assumption that we have zero leakage into the signal region. This implies that if one or more of the events seen in the data are actually leakage events, then they are contributing to the incorrect exclusion of higher mass WIMPs. The same effect can occur if we are underestimating our efficiencies at low energies. This could be a valid assumption given that we made a conservative estimate of the efficiencies based on the comparisons of the veto-coincident and calibration neutron data and simulations.

Figure 6.18 shows the calculation of the limits after excluding the four low-energy events on the assumption that these can be attributed to background leakage. This provides an upper limit on the WIMP-nucleon cross section for all WIMP masses. Figure 6.19 shows the case when the four events are included and we assume zero background leakage. As is clear from the figure, this discounts the possibility of an upper limit for low mass WIMPs. To be conservative, we need to take the upper limit of the two methods for all WIMP masses.

As indicated in the efficiency plots shown in Section 6.4.1, there is a sharp drop in the efficiency of the data cuts applied. This is a consequence of the relatively 
poor separation between events in the signal and background region at low recoil energies. As stated earlier, underestimating the efficiencies at low energies could be responsible for this case. There is the possibility that the low efficiencies of the data cuts in the 10-20 keV can cause an artificial reduction in the expected neutron spectrum at low energies which in turn leads to an apparent excess of nuclear-recoil events at low energies.

The current experimental run at the deep site indicated the absence of background events in the WIMP-candidate data set. We plan to explore fully the likely limitations of the analysis outlined in the above sections when potential backgrounds start reappearing in the experiment at the deep site. 


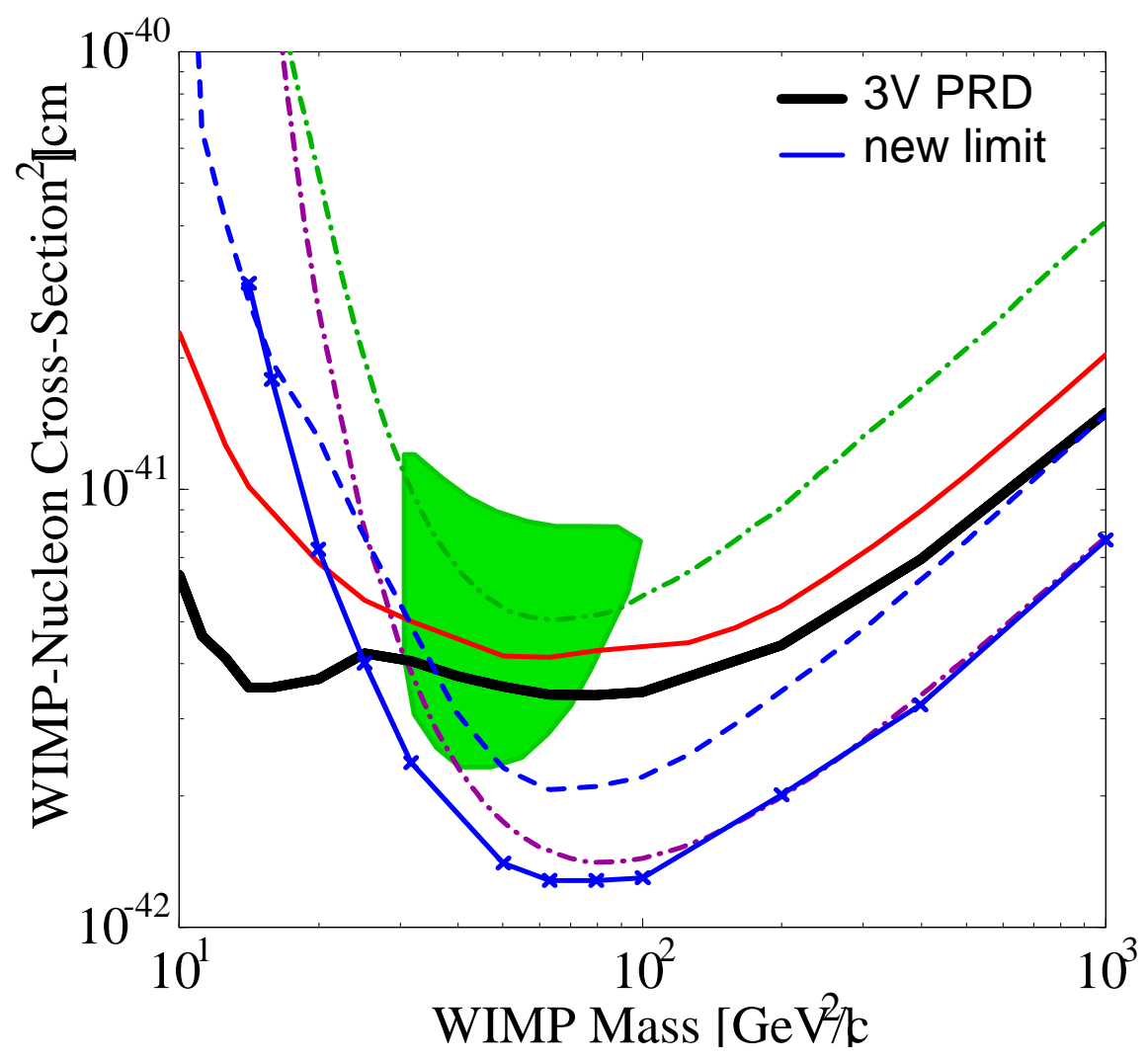

Figure 6.18: Exclusion Limits on the spin-independent WIMP-nucleon elastic scattering cross section for WIMP masses of $10-100 \mathrm{GeV}$. The solid blue line represents the limit set by the extended analysis of the Run 21 data which includes the statistical subtraction of the neutron background and under the assumption that four low energy events seen may be attributed to background leakage. The blue dashed curves shows the limits without subtraction. The black solid curve is the Run 21 $3 \mathrm{~V}$ limits after subtraction and the red solid line is the $3 \mathrm{~V}$ limit before subtraction. The purple line is the Edelweiss limit and the green closed circle represents the DAMA $3 \sigma$ allowed region. 


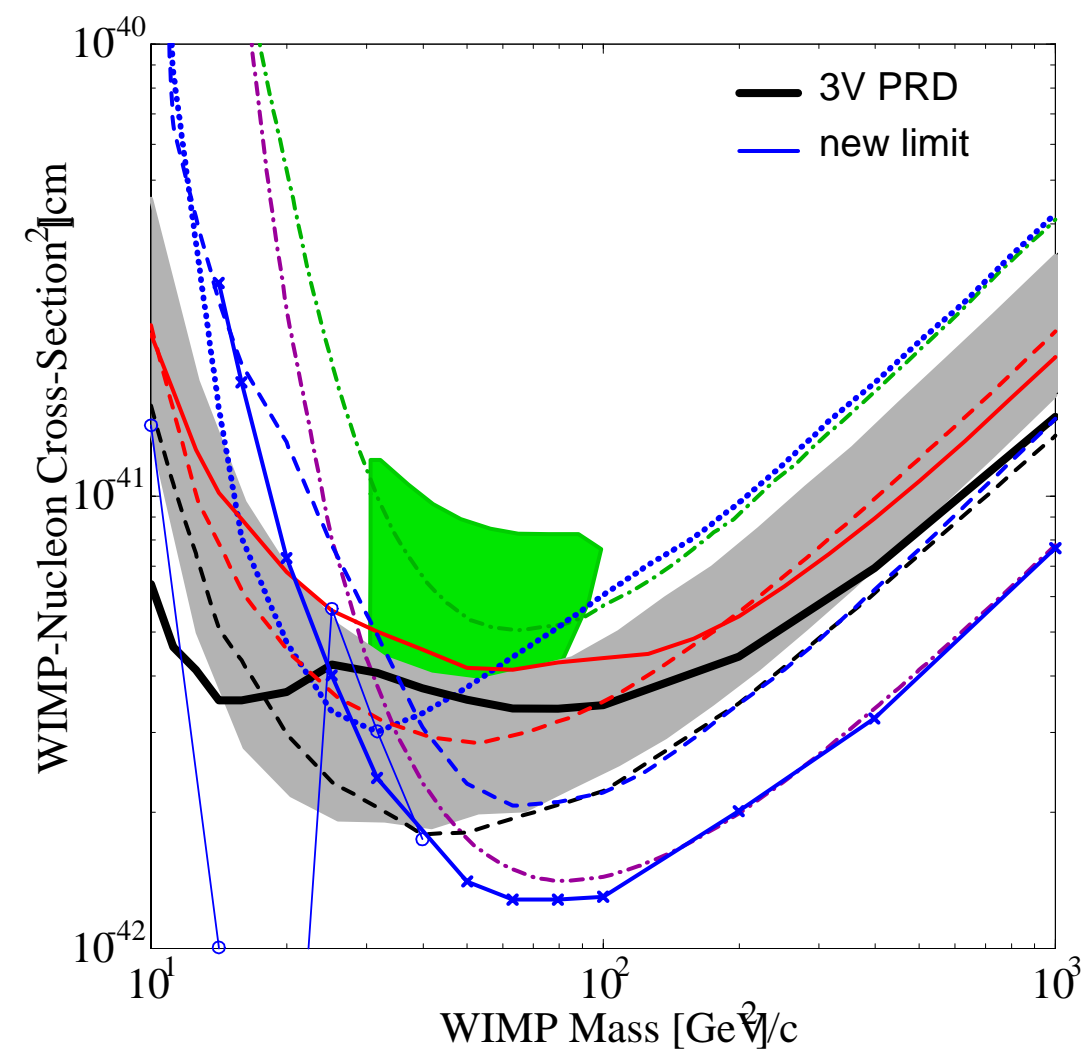

Figure 6.19: Exclusion Limits on the spin-independent WIMP-nucleon elastic scattering cross section for WIMP masses of $10-100 \mathrm{GeV}$. The solid blue line represents the limit set by the extended analysis of the Run 21 data which includes the statistical subtraction of the neutron background and under the assumption that four low energy events seen may be attributed to background leakage. The blue lines with open circles represent the limits in the event of including the four low energy events and assuming zero background leakage. The blue dashed curves shows the limits without subtraction. The black solid curve is the Run $213 \mathrm{~V}$ limits after subtraction and the red solid line is the $3 \mathrm{~V}$ limit before subtraction. The purple line is the Edelweiss limit and the green closed circle represents the DAMA $3 \sigma$ allowed region. 


\section{References}

[1] D. S. Akerib et al., Phys. Rev. D, 68, 082002 (2003).

[2] T. Saab, Ph. D. Thesis, Stanford University (2002), unpublished..

[3] D. D. Driscoll, Ph.D. Thesis, Case Western Reserve University (2004), unpublished.

[4] A. da Silva, Ph. D. Thesis, The University of British Columbia, (1996).

[5] F. F. Khalchukov et al, Il Nuovo Cimento C, 6, 320 (1983).

[6] M. Chen et al, Nucl. Instrum. E3 Meth. in Phys. Res. A, 336, 232 (1993).

[7] T. A. Perera, Ph.D. Thesis, Case Western Reserve University (2002), unpublished.

[8] D. Abrams et al, Phys. Rev. D, 66, 122003 (2002).

[9] R. Abusaidi et al, Phys. Rev. Lett., 84, 5699 (2000).

[10] http://wswww.physik.uni-mainz.de/zeitnitz/gcalor/gcalor.html

[11] Richard Schnee, private communication.

[12] V. Mandic, CDMS Run 21 Ebook Note 191.

[13] J. D. Lewin and P. F. Smith, Astropart. Physics, 6,87 (1996).

[14] R. Schnee, Run 21 Ebook Note 275 (2003). 


\section{Chapter 7}

\section{The WIMP-search at a deeper site}

A total of 968680 events were recorded by six dark matter detectors during the first CDMS WIMP-search at the 2090 m.w.e. Soudan Underground Laboratory. None of them seemed a likely WIMP, being consistent with the backgrounds seen at the site.

This chapter gives particulars of the first dark matter run - Run 118 - which took place at Soudan from October 11, 2003 to January 11, 2004. It describes the detectors used, the data acquisition techniques adopted and the data taking strategies implemented in the WIMP-search run.

It also explains how calibration runs with test sources helped define cuts that were applied to the low background data to zero in on potential WIMP candidate events and set what are currently the best ever limits on WIMP dark matter detection.

\subsection{Run 118 at the Soudan Underground Facility}

Following a year long battle with the cryogenic set-up at the 2090 m.w.e. Soudan Underground Laboratory in Northern Minnesota, the first CDMS WIMPsearch at a deep site began in October 2003. Though two towers of six detectors 
each had been installed in the Icebox prior to the cool-down to temperatures of $\sim 50$ $\mathrm{mK}$, it was decided to only run Tower 1 which had just completed a successful low background data taking run at the shallow site.

The previous chapter had shown how the presence of a limiting neutron background at the shallow site limited our sensitivity to the detection of WIMPs. The need to go deeper down persuaded the move to the 713 meter deep Soudan Underground Facility. Once an iron ore mine, the site was subsequently converted into an underground laboratory for rare event searches.

At this depth, the cosmic ray muon flux is reduced from the integrated muon flux of 44.4 muons $/ \mathrm{m}^{2} / \mathrm{s}[1]$ seen at SUF to $(2.21 \pm 0.03) \times 10^{-3} / \mathrm{m}^{2} / \mathrm{s}[2]$ here. The considerable decrease in the cosmic ray muon flux reflects in an altered profile of the backgrounds seen by the experiment at this site. While the gamma and beta rates did not show much decrease ( in fact, they registered a rise due to the increased Radon levels in the mine as explained in the next section), the neutron backgrounds fell considerably. The move underground reduced the incident neutron flux from $1 / \mathrm{kg} /$ day to $1 / \mathrm{kg} /$ year causing the background rate to fall from 1 to 0.01 events/kg-day from the shallow site to Soudan.

\subsubsection{Backgrounds seen at Soudan}

Most of the overburden of rock above the Soudan facility is made of the 3.6 million year old Ely Greenstone. The rock, which has relatively low radioactivity, has an average density of $2.75-2.8 \mathrm{~g} / \mathrm{cm}^{3}[3]$. The dominant source of backgrounds is the natural radioactivity of the rock of the experimental cavern and the materials surrounding the detector assembly. Photon backgrounds arise from the decay chains

of ${ }^{238} \mathrm{U},{ }^{232} \mathrm{Th}$ and ${ }^{40} \mathrm{~K}$. Table 4.1 in Chapter IV summarized the concentration of radioactive isotopes in the Soudan rock.

The decay of ${ }^{238} \mathrm{U}$ present in the rock produces the airborne ${ }^{222} \mathrm{Rn}$ which emanates from the walls of the cavern producing an increased gamma rate seen by 
the detectors. The measured radon levels at Soudan are $681 \mathrm{~Bq} / \mathrm{m}^{3}$, considerably higher than the $\sim 100 \mathrm{~Bq} / \mathrm{m}^{3}$ seen at the shallow site[4]. Radon levels quite typically vary significantly over time, showing a seasonal variation that causes the levels to be much higher in summer than in winter. The effect of this background is reduced by purging the surrounding with radon free old air.

The increased radon levels at the Soudan facility was partly responsible for the almost 50\% higher gamma rates seen at Soudan as compared to SUF and the consequent increase in the rate of ejectrons arising from gamma interactions with the detectors[5].

The decrease in the muon flux translates into a corresponding reduction in the muon-induced backgrounds seen at the deep site. As stated in Chapter IV, at this depth, the neutron flux is dominated by neutrons produced from the natural radioactivity of the rock and the materials of the experimental assembly. The flux due to muon-induced neutrons is more than 2 orders of magnitude below the $(2.1$ $\pm 0.2) \times 10^{-8}$ neutrons $/ \mathrm{g} / \mathrm{s}$ of the neutrons arising from natural radioactivity $[3]$. The latter, however, have a very soft spectrum with typical energies $<5 \mathrm{MeV}$ and are easily moderated by the polyethylene layers in the shielding assembly.

\subsubsection{Shielding the detectors}

The layout and composition of the shield was a consequence of the decrease in the muon flux incident on the detecting assembly and of the desire to achieve an event rate of less than 0.01 events $/ \mathrm{kg} / \mathrm{keV} /$ day from all backgrounds incident on the detectors $[6]$.

The outermost layer is a $5 \mathrm{~cm}$ thick octagonal shaped scintillator veto with 40 paddles, rejecting muons with $99.98 \pm{ }_{0.03}^{0.02}$ efficiency[7]. Each paddle is made of a plastic scintillator piece, an acrylic light guide and a Hamamatsu photo-multiplier tube. Within it is a $40 \mathrm{~cm}$ layer of polyethylene which attenuates the low-energy neutron flux arising from natural radioactivity by $1 \times 10^{6}$. Inner to this layer is 22.5 
$\mathrm{cm}$ of lead to shield the detectors against the ambient photon flux. The lead layer is a additional $5 \mathrm{~cm}$ thicker than at SUF in view of the more stringent background requirements at Soudan. It has two components - an outer layer of lead and an inner layer of low radioactivity ancient lead. This ancient lead is obtained from sunken shipwrecks as it is comparatively free of ${ }^{210} \mathrm{~Pb}$ contamination.

The lead was made internal to the polyethylene as it was a more costeffective option, and since the muon-induced neutron production in lead was much reduced here. Internal to the lead is $8.6 \mathrm{~cm}$ of inner polyethylene which, as simulations of possible shield configurations[6][8] in the early stages of the experiment indicated, effectively moderates the flux of neutron produced by muon interactions in the shield. Figure 7.1 shows a representation of the shielding assembly at Soudan while Figure 7.2 shows the geometrical configuration of the various shield layers.

\subsubsection{The Detecting Assembly}

The tower of six $250 \mathrm{~g}$ germanium and two $100 \mathrm{~g}$ silicon ZIP detectors used for Run 21 at SUF was redeployed for dark matter detection at the deep site. As noted earlier, the topmost detector in the stack suffered from poor energy resolution necessitating a higher energy threshold cut while the bottom-most detector was treated mainly as a shield given the evidence of ${ }^{14} \mathrm{C}$ contamination in it.

Run 118 saw data taken only from Tower 1 used at SUF while the current Run which began in early January at Soudan, Run 119, employs a second tower consisting of four silicon and two Germanium detectors.

\subsubsection{Cryogenics and Data Acquisition}

An Oxford Kelvinox 400-S dilution refrigerator was used to cool down the icebox and the detectors towers. The cryostat provides $400 \mu \mathrm{W}$ at $100 \mathrm{mK}$. An 


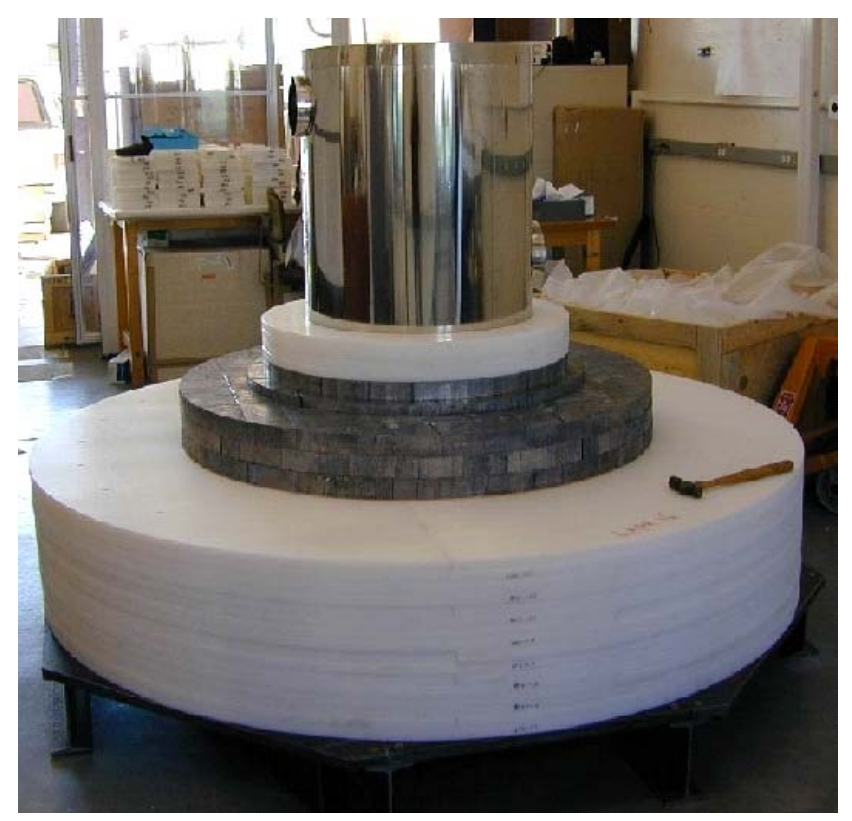

Figure 7.1: The Icebox and Shield used at the Soudan Underground Facility. The outermost layer shown is the polyethylene followed by two layers of lead, an inner polyethylene and the icebox cans. The scintillator muon veto which is external to the outer polyethylene is not shown in the diagram. 


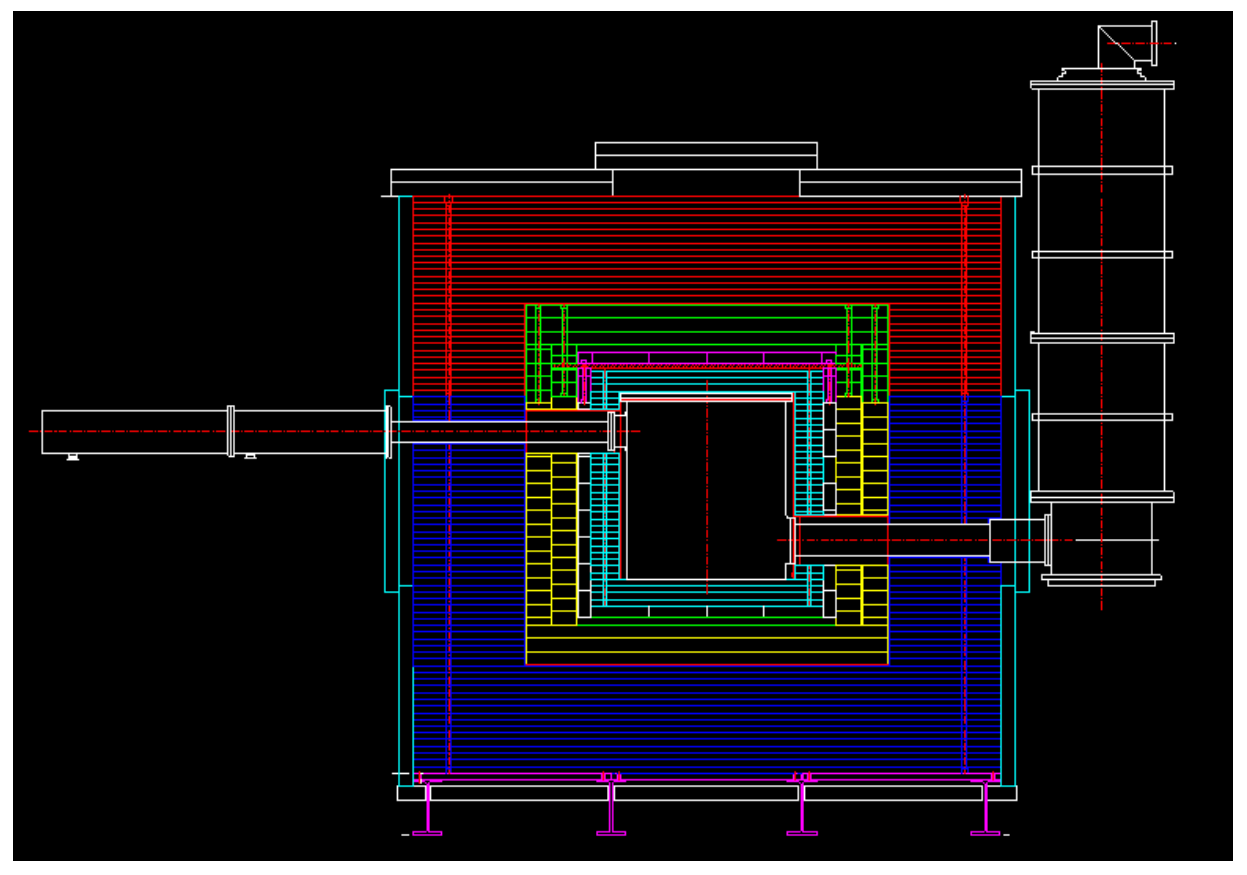

Figure 7.2: Icebox and shield with cryostat. Shown in the sketch is the scintillator muon veto, the polyethylene and lead layers of the shield, the icebox and cryostat. Also shown are the E-stem that connects the icebox to the electronics and the C-stem that links it to the dilution refrigerator unit shown. 
Icebox consisting of concentric copper cans cooled to progressively lower temperatures of $4 \mathrm{~K}, 600 \mathrm{mK}, 50 \mathrm{mK}$ and $10 \mathrm{mK}$ house the detector assembly in a radio-pure environment.

Once the system is cooled down to temperatures of $\sim 50 \mathrm{mK}$, regular Liquid Helium and Liquid Nitrogen transfers are necessary to keep the system at these very low temperatures. Since access to the mine is limited, the process is automated using a computer based control and monitoring unit with an 'Intellution' (Intelligent Solution) software interface. This allows for cryogenic operations to be conducted by computer control inside the mine or at the surface when it is not possible to be underground. An Intelligent Gas Handling System controls the cryogenics associated with the dilution unit that cools the system below $4 \mathrm{~K}$.

The data acquisition system (DAQ) in operation at Soudan is geared to handle the full complement of seven towers with 42 detectors that was part of the initial WIMP-search plan at the underground facility. Like the cryogenic handling systems, the DAQ was designed to be remotely operated given the constraints on mine access. It has a higher bandwidth and is capable of handling data throughput at the designed rate of $10 \mathrm{~Hz}[9]$. Like the cryogenic systems, the DAQ, too, has the facility of being remote controlled for detector tuning, monitoring and data acquisition either from the mine or from the surface or from hundreds of miles away from the experimental set-up.

Data was acquired on a regular basis for the entire duration of the run except during cryogen transfers and in the event of cryostat related problems. This data was then analyzed using the data analysis software package called DarkPipe on a Linux cluster consisting of 10 analysis nodes. The cluster can analyze up to 10 events/s during a typical run. DarkPipe analyses the data, producing reduced quantities (RQs) such as amplitudes of the phonon and charge pulses of an event, the start time and rise time of such phonon pulses, amplitudes of the veto pulses, trigger information and other parameters necessary for the dark matter analysis. Software routines such as PipeCleaner pick up these RQs and generate RRQs (reduced reduced quantities) which provide complete information of an event in terms 
of energy deposited in $\mathrm{keV}$, position information in terms of delay parameters in $\mu s$ and rise time of the pulses, also in $\mu s$.

\subsubsection{Time line of the Run}

It took approximately three years for the cryogenic systems to be made fully operational before the start of the cool-down in April 2003. After a couple of cryogenic hiccups along the way, the detecting assembly was cooled down to a temperature of $<50 \mathrm{mK}$. Over 968,000 events were taken during the first WIMPsearch run which amounted to 52.6 Live Days of data after cutting out periods of poor noise, cryogenic fills and detector related problems. Figure 7.3 indicates the efficiency of the accumulation of data during the period of the run. The efficiency recorded averaged $67 \%$ over the entire run period, with the last six weeks recording over $85 \%$ efficiency.

\subsubsection{Neutralizing the detectors}

The beginning of the data run at Soudan coincided with noise debugging and tuning the detectors for the dark matter search. Initial studies included neutralization to fill impurity sites in the detectors that can lead to poor charge collection which in turn affects the event discrimination. The detector physics necessitating the neutralization was explained in Chapter III. For the Soudan set-up, neutralization proceeded by periodic flashing of LED with the flashing occurring for about a minute every 12 hours followed by a waiting period to allow the detectors to cool back down to base temperatures.

\subsubsection{Calibration runs with the ${ }^{133} \mathrm{Ba}$ source}

The ionization voltage bias used for the germanium detectors was $-3 \mathrm{~V}$ while $-4 \mathrm{~V}$ was used for the silicon detectors. The choice of the bias was dictated by the 


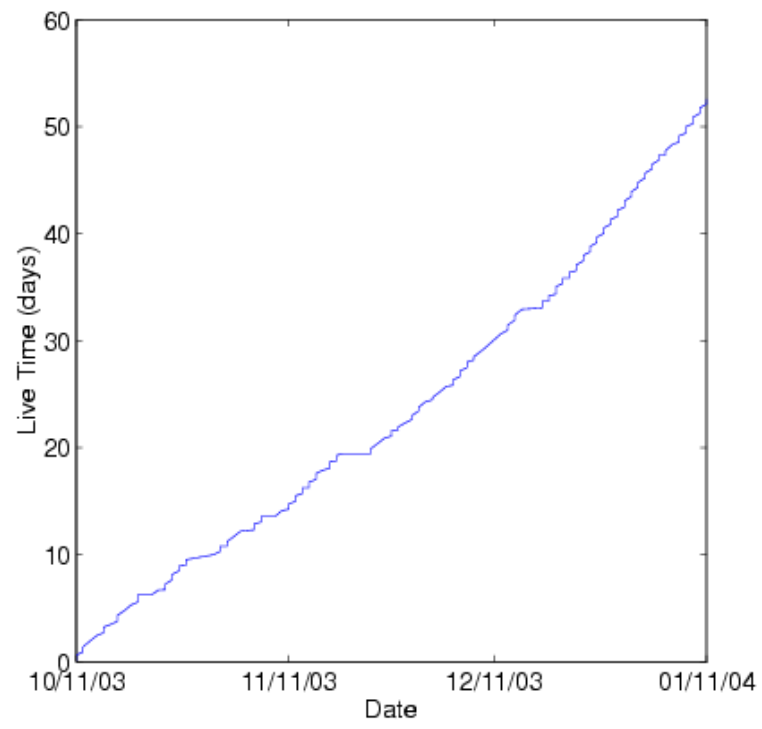

Figure 7.3: Efficiency of the accumulation of WIMP search data for the duration of the first Soudan run. Here Live Time of the run is plaotted against real time (taken from [10]).

performance of a ZIP detector G31 in a test facility run when different charge biases were tested for optimal discrimination against surface beta events[10].

A ${ }^{133} \mathrm{Ba}$ source was used both for setting the energy calibration scale for the electron-recoil events as well as providing a large sample population of electronrecoil events to define the background regions for the detectors. The $2 \mu \mathrm{Ci}{ }^{133} \mathrm{Ba}$ source was inserted in position through a special tube along the E-stem of the Icebox to allow the source to penetrate the lead layers in the shield. The source gives distinct lines at 356 and $384 \mathrm{keV}$, allowing for its use to set the energy calibration scale for the charge channels of the detector. The energy scale was set comparing the output of the detectors to a Geant3 Monte Carlo simulation of the gamma calibration run.

Figures 7.4 and 7.5, taken from [11] shows the comparison of data and simulations for the ${ }^{133} \mathrm{Ba}$ calibration run. The comparison is shown for the charge channels which show a linear response in this energy range, unlike the phonon 


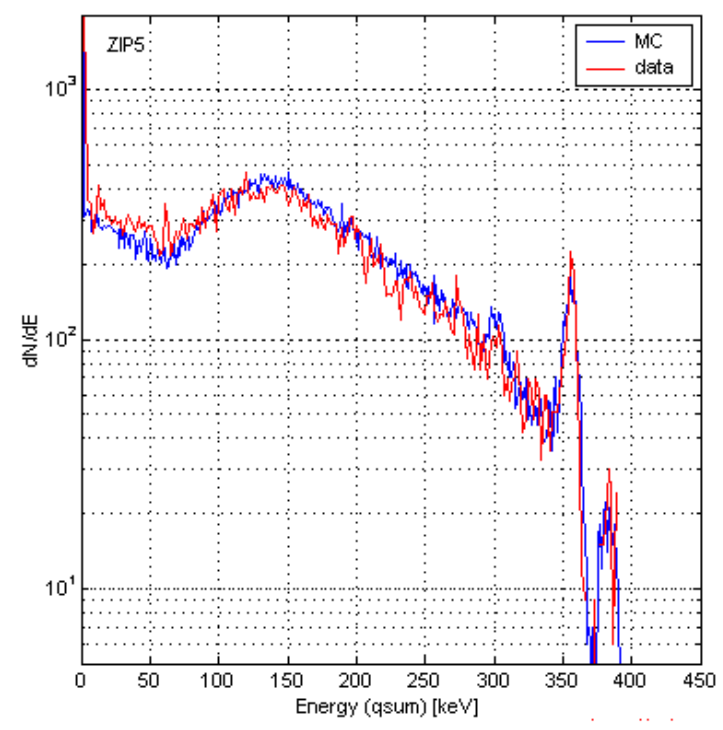

Figure 7.4: Comparison of data and simulations for the ${ }^{133} \mathrm{Ba}$ calibration run. The comparison for the ionization channel energies show clear peaks at $356 \mathrm{keV}$ and 384 $\mathrm{keV}$. The detector is Z5, a germanium detector.

channels which show non-linear behavior above $200 \mathrm{keV}$. For the charge channels, the germanium detectors show clear peaks at $356 \mathrm{keV}$ and $384 \mathrm{keV}$. The lines are less discernible in the silicon detectors on account of the larger penetration depth of photons in silicon as compared to germanium. In the case of silicon, therefore, the comparison in done in terms of the spectral shapes in the absence of peaks. Once the charge channels are calibrated, they in turn are used to calibrate the phonon channels.

\subsubsection{Event discrimination}

The ZIP detectors demonstrated $99.98 \%$ rejection capability against gamma and bulk electron events in Run 21[12]. For the Soudan run of the same tower, there was excellent discrimination against electron-recoil events arising from photons and 


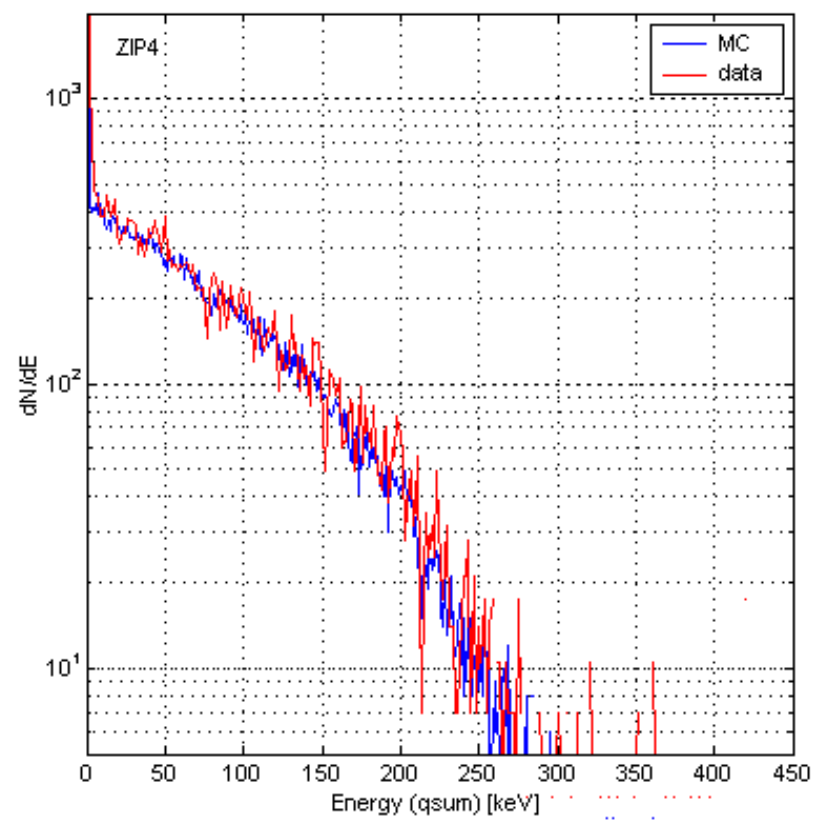

Figure 7.5: Comparison of data and simulations for the ${ }^{133} \mathrm{Ba}$ calibration run. The comparison is for the ionization channel energies for the silicon detector Z4. 
electrons interacting in the bulk of the crystal. The gamma leakage for the 52.6 live days of Run 118 is estimated at $<0.25$ events at $90 \%$ CL[10].

Electrons which interact within a few $\mu m$ of the detector's surface where there is poor charge collection give rise to low-yield events that may be mistaken for nuclear-recoil candidates. Most of such events arise from low-energy electrons produced either from contaminants on the detector surface or as a consequence of gammas interactions with a detector ejecting electrons that have surface interactions with the neighboring detector. This second class of events has been called

ejectrons and studies have sought to evolve optimal methods to discriminate against them. This included a calibration run with a ${ }^{109} \mathrm{Cd}$ beta source and a germanium detector G31 in a test facility run[13] to determine optimal charge bias and better rejection methods against this class of events.

Timing parameters such as the variation of the rise time of the phonon pulse and the delay between the charge and phonon pulses are used to develop data cuts to reject surface electrons. The discussion on the veto anti-coincident nuclear-recoil data set explains how such cuts helped reject this contribution to the signal region

\subsection{Defining the fiducial-volume cut}

The fiducial-volume cut selects events that fall in the inner electrode region alone as events which may occur in the outer guard electrode may suffer from poor charge collection affecting the event discrimination of the signal. For Run 118, the fiducial-volume cut was defined by examining the charge energies in the qinnerqouter plane. Here qinner and qouter refer to the amplitudes of the charge pulses in the inner and outer charge electrode regions.

After folding in the corrections for the cross talk and position dependence in the charge energies, energy bins of [10 20], [30 40], [50 60], [70 80] and [90 100] were selected in qinner and histograms plotted of these energies[14]. Gaussian fits 
were applied to the histograms and the mean and standard deviation of each fit determined.

The fiducial-volume region was selected as a band in the qinner-qouter plane where the upper bound of the band was defined by (mean $+\sigma+0.04 \mathrm{x}$ qinner) for each bin and the lower bound given by (mean $-3 \sigma$ ). Figure 7.6 illustrates how the fiducial-volume cut is defined. The $3 \sigma$ band is adequate for defining the lower bound. For the upper bound, however, we select the band as (mean $+\sigma+0.04 * q i)$ to ensure that we do not cut off genuine inner electrode events at higher energies and to reject shared events in the lower energy regions. A similar $3 \sigma$ band for the upper bound would have included such shared events at low energies.

The efficiency of the cut was then tested for neutrons using the neutron calibration data. The efficiency was also determined using the output of neutron simulations for the calibration runs. Poor statistics in the neutron calibration data at higher energies and leakage of gamma events into the signal band in the qouter region leads to a fall in the efficiency of the cut at higher energies. For the data, this cut is applied selecting events in the signal region which pass the data quality cuts and the ratio of events passing the qinner cut to all such events determined over the entire energy range. Leakage of gamma events in the signal region do not allow for a genuine pre-selection of nuclear-recoil events, and reflects in a decreased efficiency at higher energies, as indicated in Figure 7.7.

Determining the efficiency of the cuts using the simulated output of a neutron calibration run allows for a pre-selection of bona-fide nuclear-recoil candidates on which the efficiency of the fiducial-volume cut may be determined and allows for making a conservative estimate of the efficiency for determining the exposure of the run. This procedure is explained in the next section. 

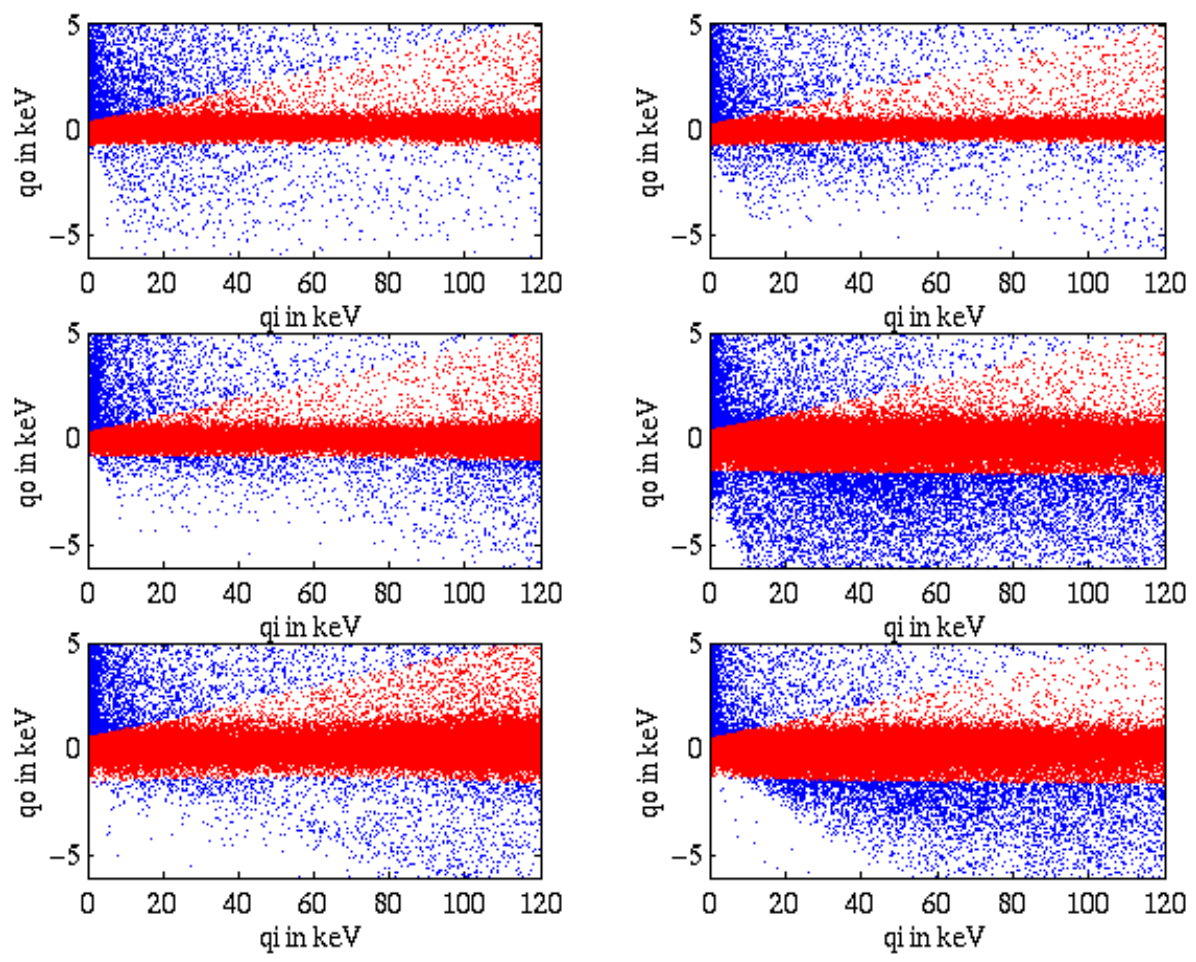

Figure 7.6: Defining the fiducial volume cut for the Run 118 WIMP search run. Events in red are those selected to lie in the fiducial region. The choice of the upper bound of the band is to allow for the inclusion of bonafide qinner events at higher energies while rejecting likely shared events at low energies. 

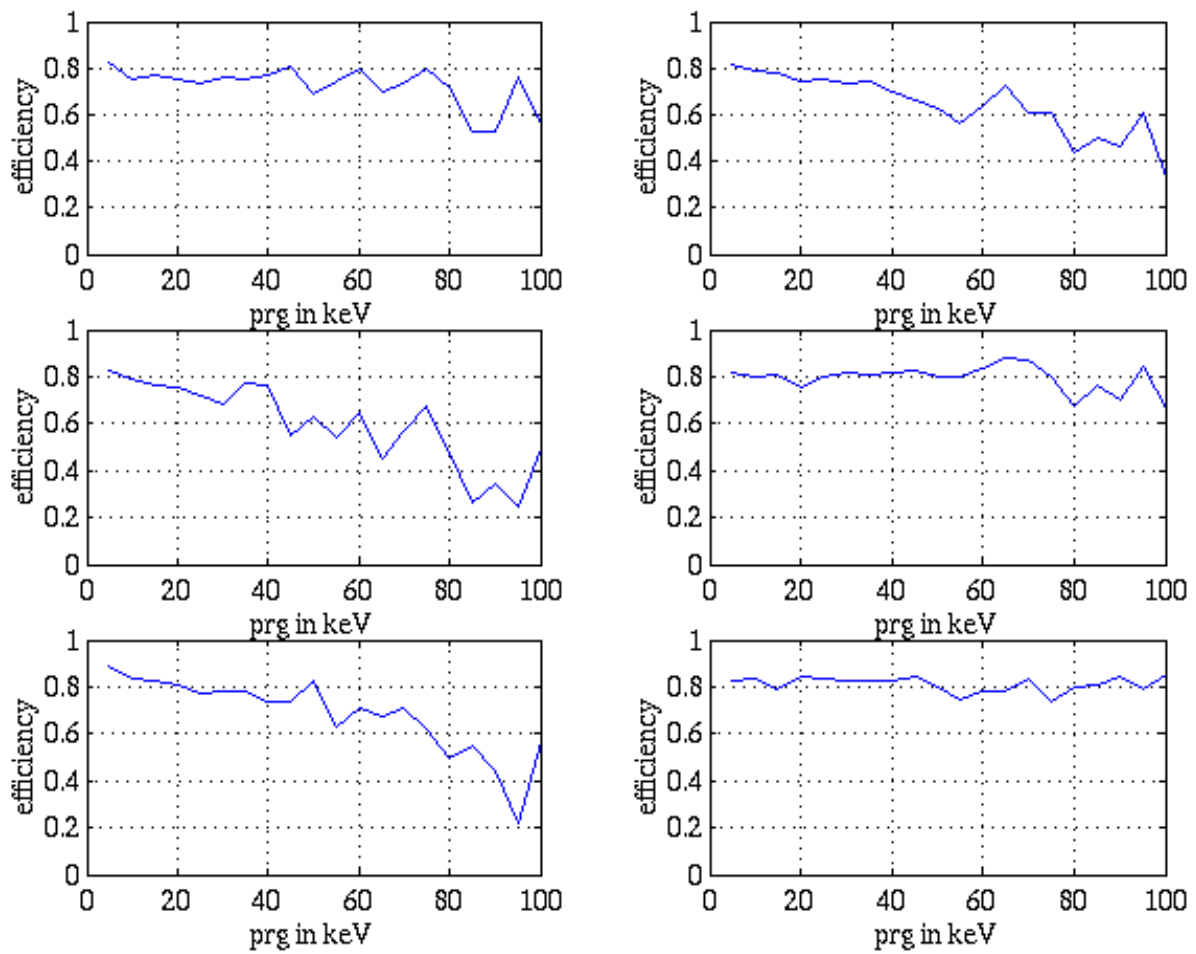

Figure 7.7: Efficiency of the qinner cut as determined from the neutron calibration data. The fall at higher energies in the germanium detectors is on account of gamma leakage in the outer region contaminating the sample of bonafide nuclear recoil events coupled with poor statistics in the calibration runs at higher energies. The effect is most pronounced in Z5. 


\subsection{Neutron calibrations with the ${ }^{252} \mathrm{Cf}$ source}

The neutron calibration runs define a signal region where potential WIMP candidates may be detected. For the first data run at Soudan, the signal region was defined by calibrations with a $5 \mu \mathrm{Ci}$ neutron source. The source emits 22,940 neutrons/s in a typical fission spectrum with a peak energy of $\mathrm{MeV}$ as specified in Chapter V.

For the Soudan analysis, GEANT 3 simulations were undertaken for two purposes. Prior to the start of the run, the simulations sought to identify optimal positions of the source to achieve a good event rate in the detectors and collect enough statistics to define the signal regions while minimizing possible activation of the detectors due to the source. Once the source position was decided, and calibration runs undertaken, the simulations were used to compare rates and spectra for data and modelled output.

Unlike the case of the shallow site, making sure there was reasonably good agreement between data and simulations was not as critical here. The absence of a discernible neutron background made it unnecessary to make a statistical subtraction of the neutron contribution as in the case of Run 21.

The simulations of the calibration runs were used to check the efficiencies of the various data cuts. In particular, the Monte Carlo simulations were used to make estimates of the efficiency of the fiducial-volume cut applied to the Soudan data as will be detailed in a subsequent section.

\subsubsection{Coding in the geometry}

The shield surrounding the Soudan Icebox had a different configuration as compared to the one used for Run 21 in view of the different background profiles at the two experimental sites. Unlike the case of the shallow site, there were no layers of the shielding internal to the copper cans of the icebox due to the reduction in 
the incident neutron flux and the need to put a full complement of seven towers (subsequently scaled down to five) inside the icebox. As can be seen, two towers were installed though only one was operational during Run 118. The electronics stem that connects the icebox to the readout systems (E-stem) and the cold stem that allows for the cooling of the icebox by the dilution refrigerator (C-stem) are shown.

The source hole for the neutrons is the same as that for the ${ }^{133} \mathrm{Ba}$ source, consisting of the plastic tube running parallel to the length of the E-stem as indicated. The neutron source is housed in a double sealed stainless steel capsule with a stainless steel wire welded to it. The source is inserted into the plastic tubing and moved along the length of the tubing using the steel wire. We are thus allowed only one degree of latitude for changing source position vis-a-vis the detector assembly.

\subsubsection{Identifying optimal source positions}

Simulation of test runs with the source in different positions were tried out to arrive at an optimal event rate in the detectors for a short duration neutron calibration run. Placing the source very close to the detectors increases the fraction of direct scatters as compared to interactions from neutrons that have bounced around before hitting the detectors. The direct scatters give higher characteristic energies. The spectrum of these initial neutrons is harder with average energies of the order of $2.3 \mathrm{MeV}$ as compared to neutrons that scatter first in the shield which have average energies around $700 \mathrm{keV}$.

The simulations used the GEANT 3 code with neutrons thrown at the source rate of 22,940 neutrons/s and a spectrum that follows the Californium fission spectrum. At the end of the source hole tubing is the closest possible position of the source. The event rate, with no energy cuts is over 100 events/sec while those with recoil energies $5-100 \mathrm{keV}$ occur at a rate of $70 / \mathrm{s}$, clearly too high a rate for the data acquisition system to handle. Figure 7.10 shows the results of simulations 


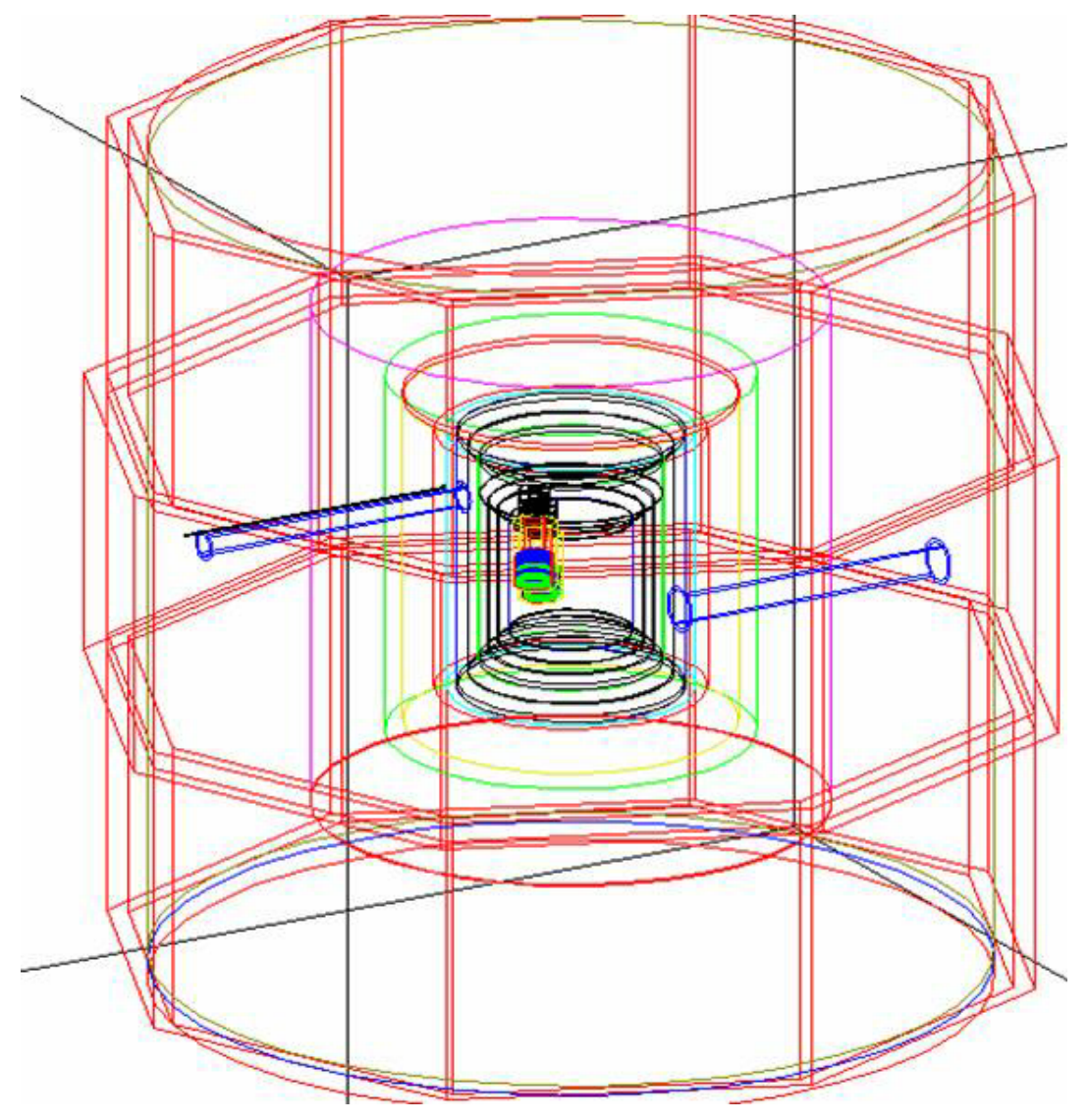

Figure 7.8: Monte Carlo representation of the shield and detecting assembly used for Run 118 at Soudan. Moving inwards is the octagonal scintillator muon veto with 40 paddles, the outer polytheylene, the two lead layers, the inner polyethylene, the copper cans of the icebox and the tower and detector assembly. 


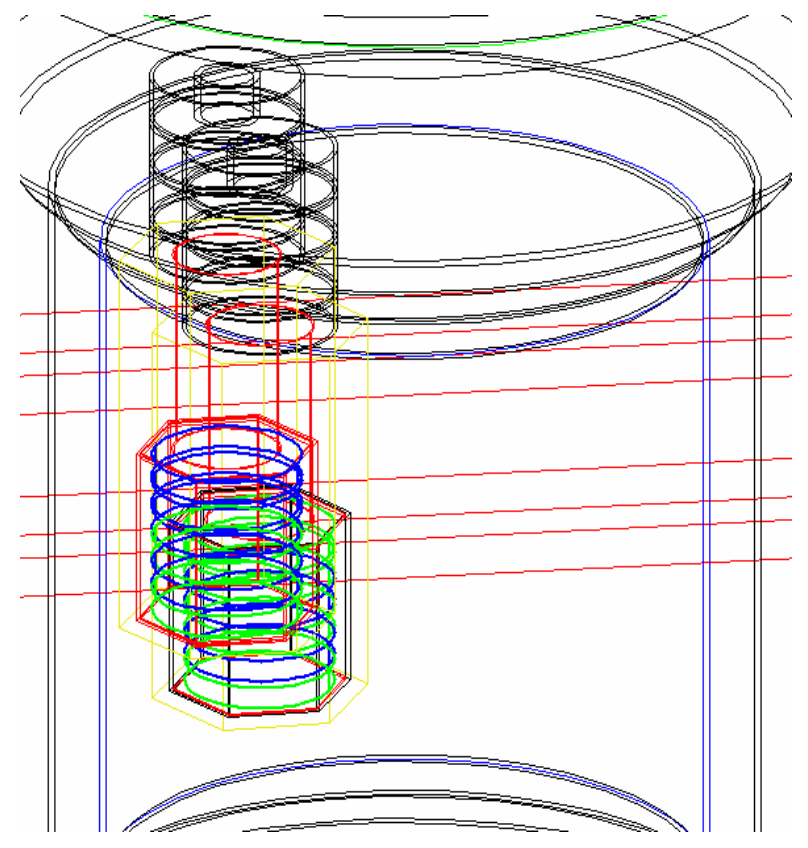

Figure 7.9: Close-up of the towers and detecting assembly

to study the variation in the event rate on the detectors for different distances of the source from the detector assembly.

Based on the simulations studies, a source position was chosen such that the total event rate without any energy cuts was 6.8 events/s and fell to 3.8 events/s for energies between $10-100 \mathrm{keV}$. Coupled with the gamma rate either from direct gammas emitted by the source ( a negligible value) and $2.2 \mathrm{MeV}$ photons produced from neutrons falling below the $10 \mathrm{keV}$ thermal energy threshold in the polyethylene, this provided an event rate within the upper limit of $10 / \mathrm{s}$ for the DAQ to handle. For the Run 118, this was the source position chosen for the ${ }^{252} \mathrm{Cf}$ source.

\subsubsection{Defining the signal region}

Three neutron calibration runs took place during the course of Run 118. We use the calibrations of December 10, 2003 and January 5, 2004 to define the signal 


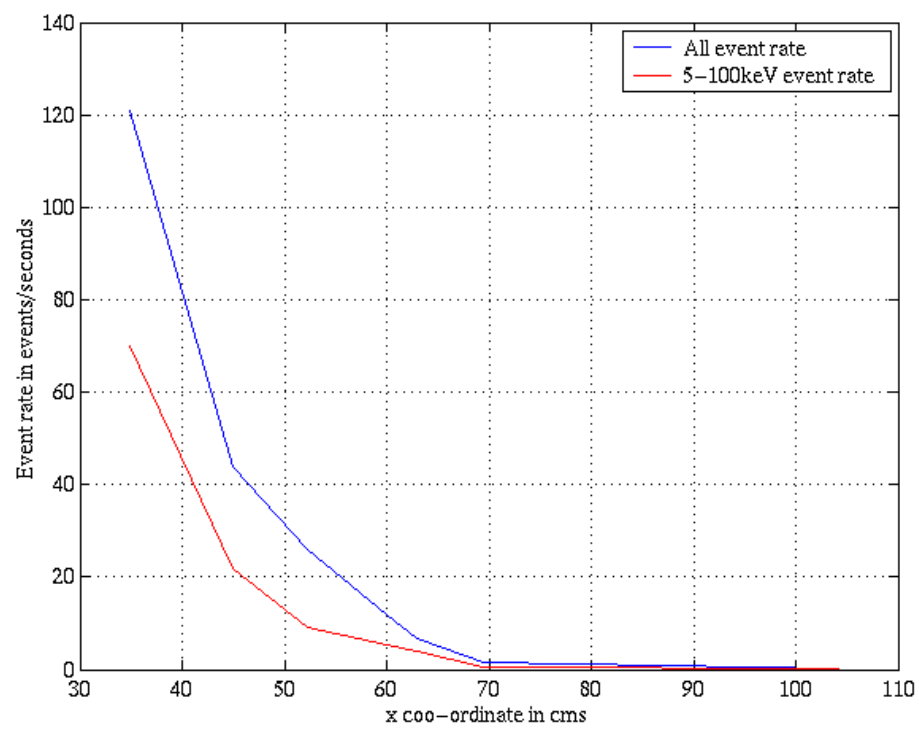

Figure 7.10: Variation of the event rate seen in the detectors with the distance of the ${ }^{252} \mathrm{Cf}$ source from the detecting assembly.

regions and select events passing the nuclear-recoil band. Appendix A gives details on the band fitting routines used in selecting the signal region. Figure 7.11 shows yield as a function of energy for the second and third neutron calibration data runs indicating the bands that define the signal regions.

\subsubsection{Data cuts}

To compare data and simulations for the two neutron calibration runs considered, we had to fold in the cut efficiencies of the data to the output of the simulations. Cuts were formulated selecting events based on the neutron and gamma calibration data. Defining the cuts for the first Soudan data was a combined effort of the collaboration. For the data, the following cuts were used:

- A cut rejecting all bad data, i.e., events triggered by noise glitches, with poor signal to noise in particular phonon channels, with no global trigger recorded 

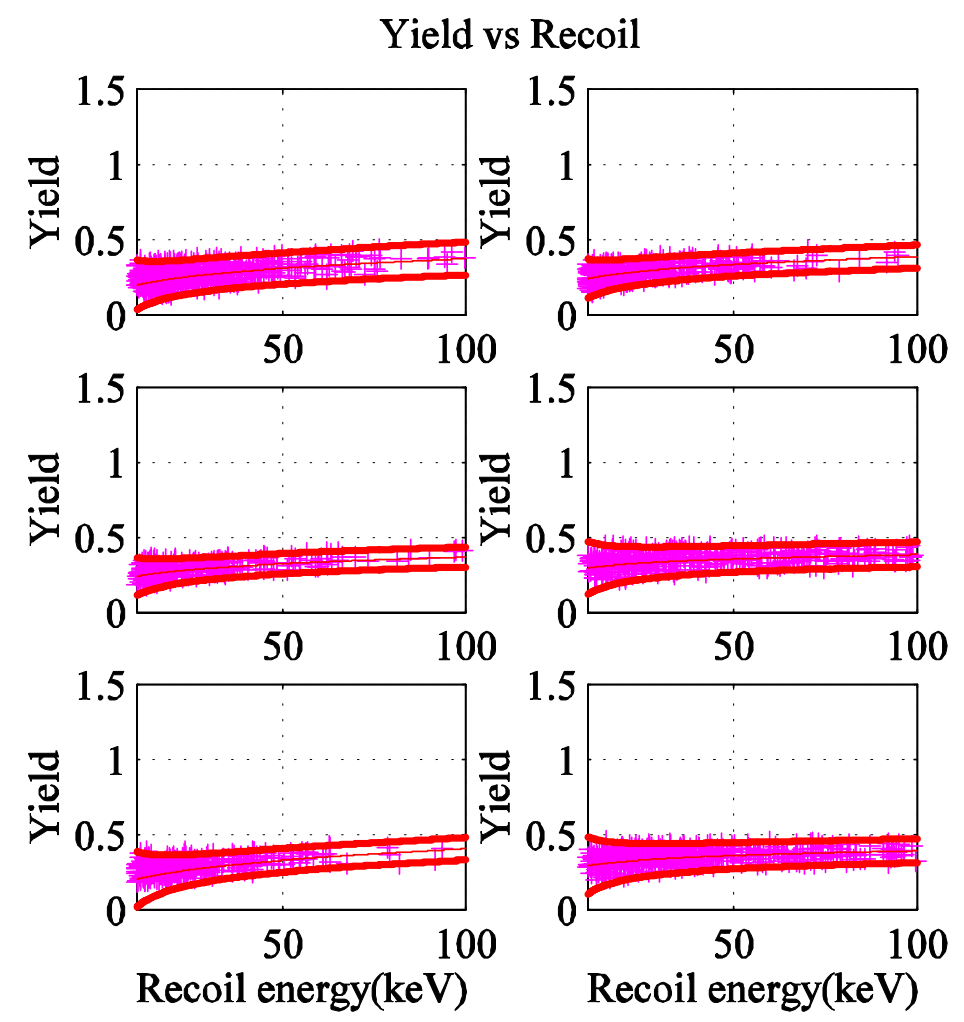

Figure 7.11: Yield as a function of recoil energy for the neutron calibration. The red crosses indicate single scatter nuclear recoil events in the detectors 
in the history buffer and events occurring during cryo transfers.

- Cuts rejecting pile up events and those triggered by noise glitches as defined by the $\chi^{2}$ of the fit to the charge channels and the standard deviation of the pre-trigger part of the phonon pulses.

- Cuts to define the threshold energies for the charge and phonon channels. The charge threshold cut ensure that events with no charge signal are not mistaken for low-yield events by selecting events with a measurable pulses in the charge channels. Analysis thresholds of $10 \mathrm{keV}$ were set for all but the top-most detector $\mathrm{Z} 1$ which was given a higher threshold of $20 \mathrm{keV}$ on account of its known poor energy resolution.

- The fiducial-volume cut rejecting events that fall in the outer guard electrode of the detectors. The procedure for selecting these events was explained earlier.

- The nuclear-recoil band cut selecting events that fall in the signal region.

- The singles cut selects events which have phonon signals $6 \sigma$ above the noise in only one detector.

- The multiples cut defines an event as a multiple if all detectors pass the data quality cuts and two or more detectors pass the phonon threshold and fiducial-volume cuts. For the case of nuclear-recoil candidates, two or more scatters should also fall in the signal band.

In addition, there were other data cuts formulated which were applied to the WIMP-search data. They include:

- A rise time cut that depends on the rise time of the phonon pulse and the start time of the phonon pulse relative to the ionization signal. This cut aims at rejecting surface electron-recoil events that can be mistaken for signal events 
due to their low charge yield. The rise time cut cRT2S minimizes (Po90/ $\alpha$ ) where Po90 is the Poisson 90\% upper limit on the expected number of surface events likely to leak into the signal region after applying the phonon timing cut to the WIMP-search data[10]. For surface events, both parameters are much shorter as opposed to genuine nuclear-recoil events.

- A muon veto cut that selects all events in the detectors for which the time to the most recent trigger in the muon veto scintillator is more than $50 \mu \mathrm{s}$.

The simulations of the neutron calibration runs produce a population of nuclear-recoil events in the detectors. The corresponding charge energies and yield are determined in a manner similar to that outlined in Chapter V. The recoil spectra were compared detector wise for all events passing the nuclear-recoil band cut and then for the single-scatter events. Figures 7.12 and 7.13 show the comparisons and Table 7.1 gives the event rates for single-scatter nuclear-recoil events in all six detectors. 

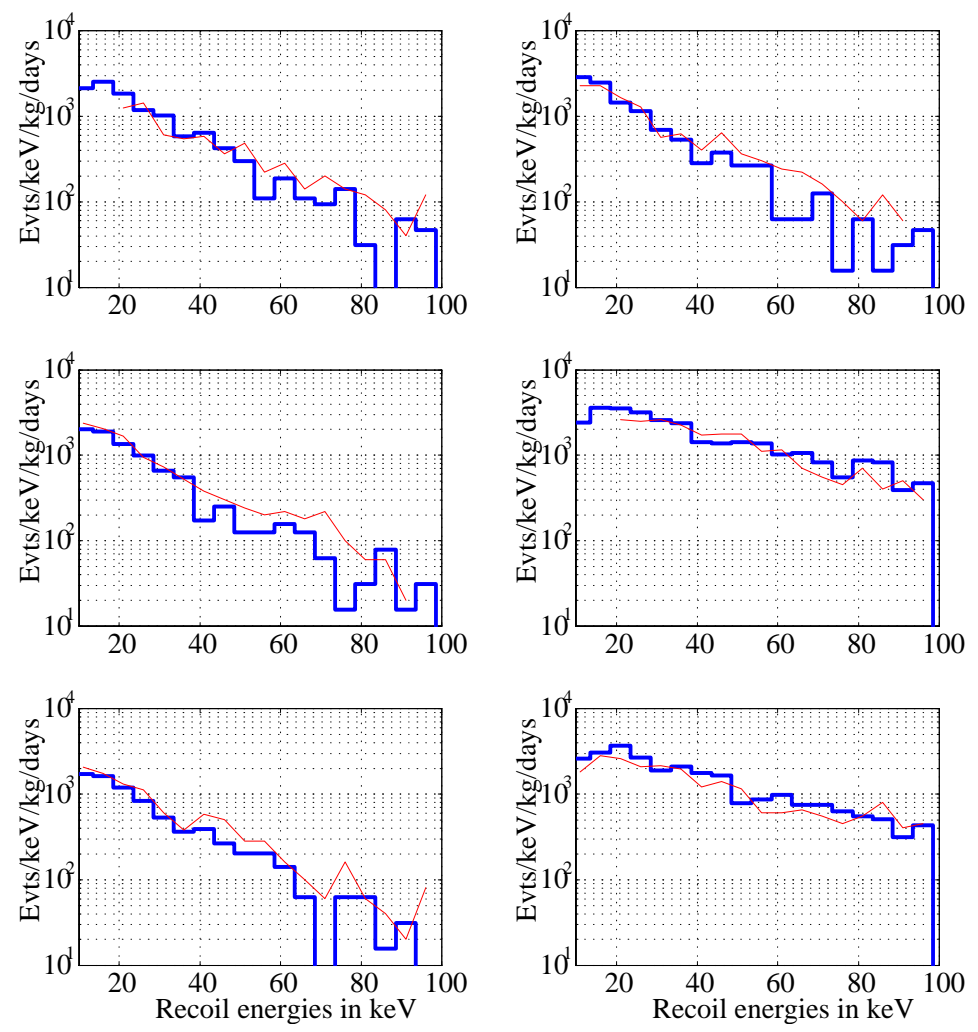

Figure 7.12: Comparisons of data and simulations for the neutron calibration run. Case of all events passing the nuclear recoil band cut. 

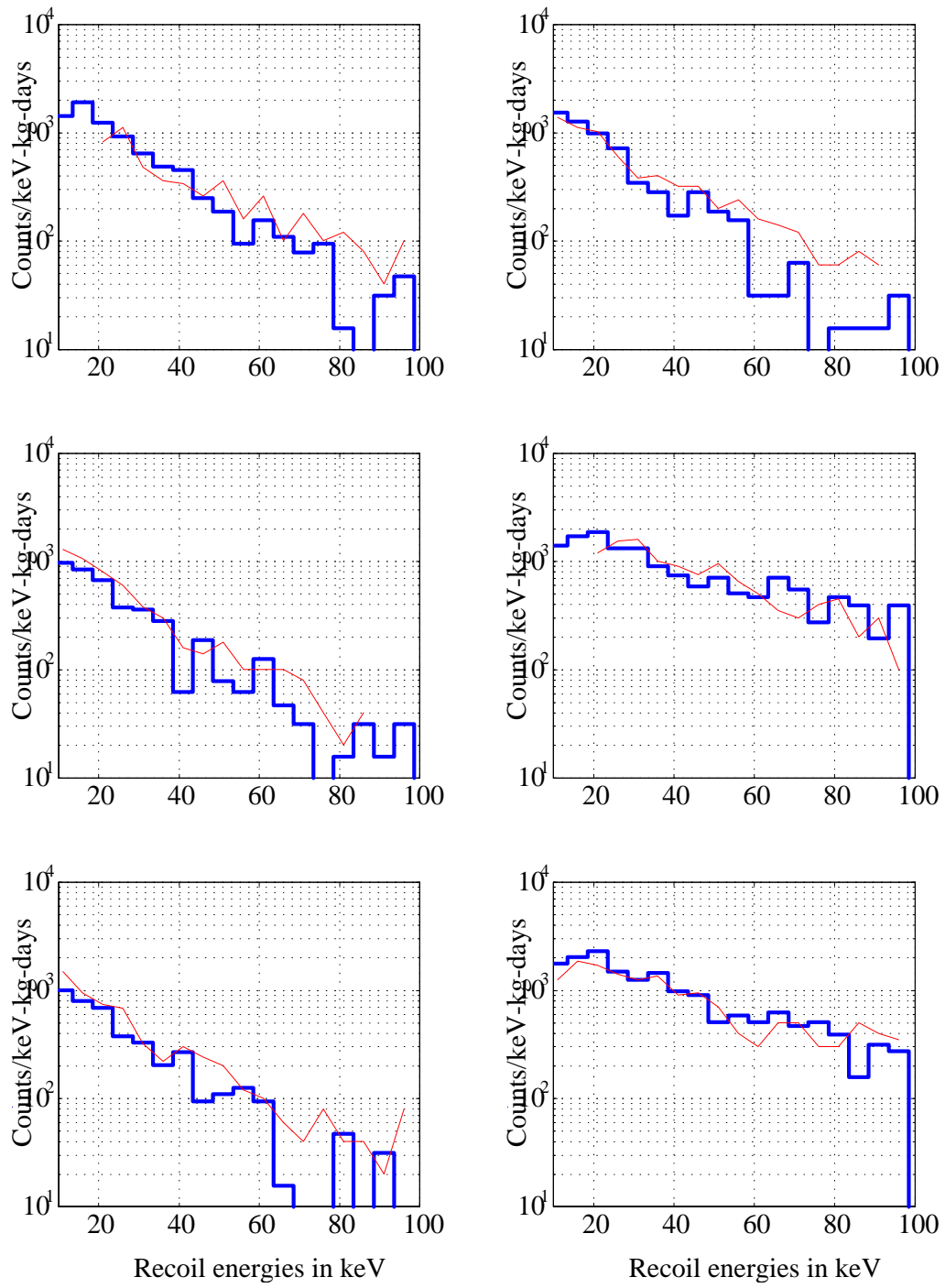

Figure 7.13: Comparisons of data and simulations for the neutron calibration run. Case of single scatter events passing the nuclear recoil band cut. 


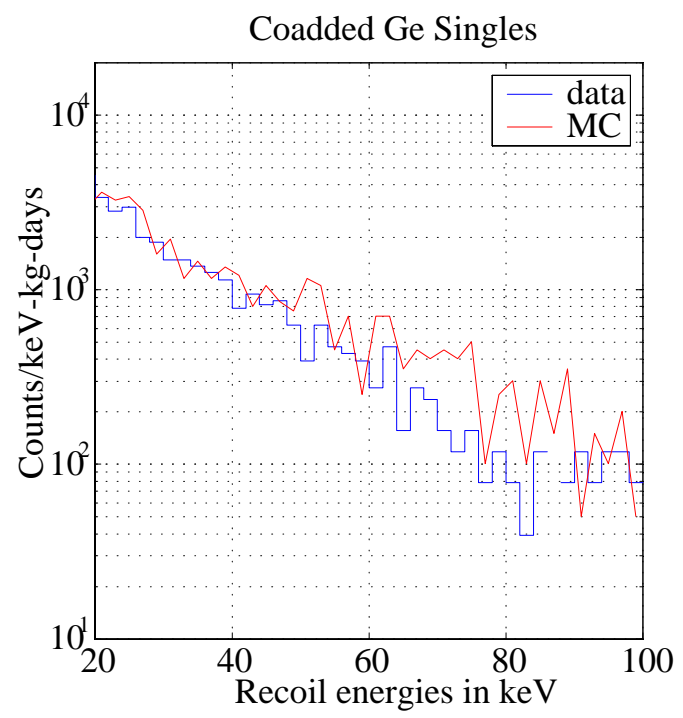

Comparison of single scatters for the coadded Ge detectors

Coadded Si Singles

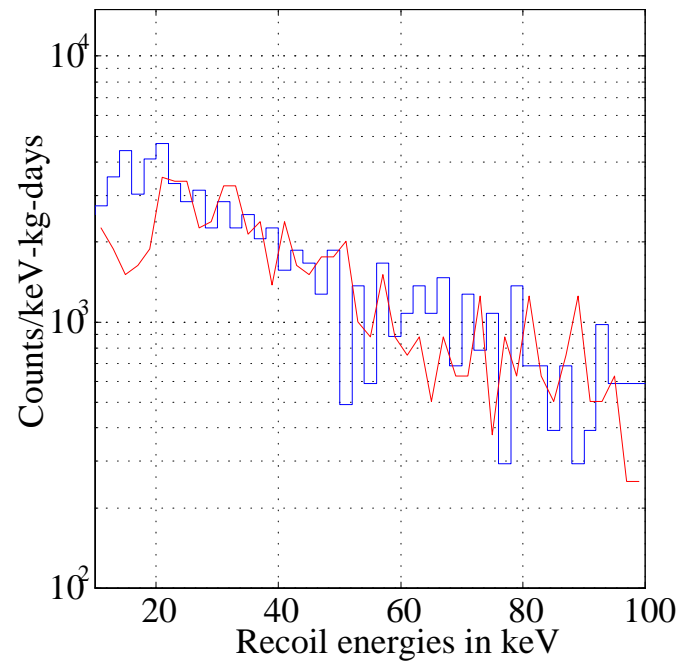

Comparison of single scatters for the coadded silicon detectors.

\subsection{Analysis of data from the first Soudan WIMP- search run}

A WIMP-search run consists essentially of reducing an entire data set of events taken under low background conditions to a subset of dark matter candidates. For 


\begin{tabular}{|c|l|l|}
\hline Evts/kg/day & singles(data) & singles $(\mathrm{MC})$ \\
\hline Z1 & $2.2 \pm 0.1 \times 10^{4}$ & $2.6 \pm 0.2 \times 10^{4}$ \\
\hline Z2 & $2.8 \pm 0.1 \times 10^{4}$ & $3.5 \pm 0.2 \times 10^{4}$ \\
\hline Z3 & $1.9 \pm 0.1 \times 10^{4}$ & $2.8 \pm 0.2 \times 10^{4}$ \\
\hline Z4 & $5.5 \pm 0.3 \times 10^{4}$ & $5.9 \pm 0.4 \times 10^{4}$ \\
\hline Z5 & $1.9 \pm 0.1 \times 10^{4}$ & $3.0 \pm 0.2 \times 10^{4}$ \\
\hline Z6 & $8.0 \pm 0.4 \times 10^{4}$ & $7.9 \pm 0.5 \times 10^{4}$ \\
\hline
\end{tabular}

Table 7.1: Event rates for all events and single scatter events detector-wise for the Run 21 3V neutron calibration. The efficiencies of the standard 3V cuts have been folded into the Monte Carlo simulations.

the Run 118, it was decided to do a blind analysis. This meant that the signal region in the WIMP-search data was not examined until all the data cuts had been defined on the gamma and neutron calibration data. The rationale behind this is that possible bias in setting cuts is eliminated by such an approach. The cuts defined for the WIMP-search analysis in Run 118 were specified in the previous section. The cuts were applied to the WIMP-search data to zero in on potential WIMP-candidate events

\subsubsection{WIMP candidate events}

The WIMP_candidate data set refers to the single-scatter nuclear-recoil events seen in the four germanium detectors. When this data set was thrown open to purview, after application of all the cuts set on the calibration data sets, we found that no single-scatter nuclear-recoil event in the germanium detectors passed the data cuts. Shown below is the plot for the anti-coincident events in all six detectors with the rise time cut applied. 
Veto-anticoincident single scatter nuclear recoils - after rise time cut
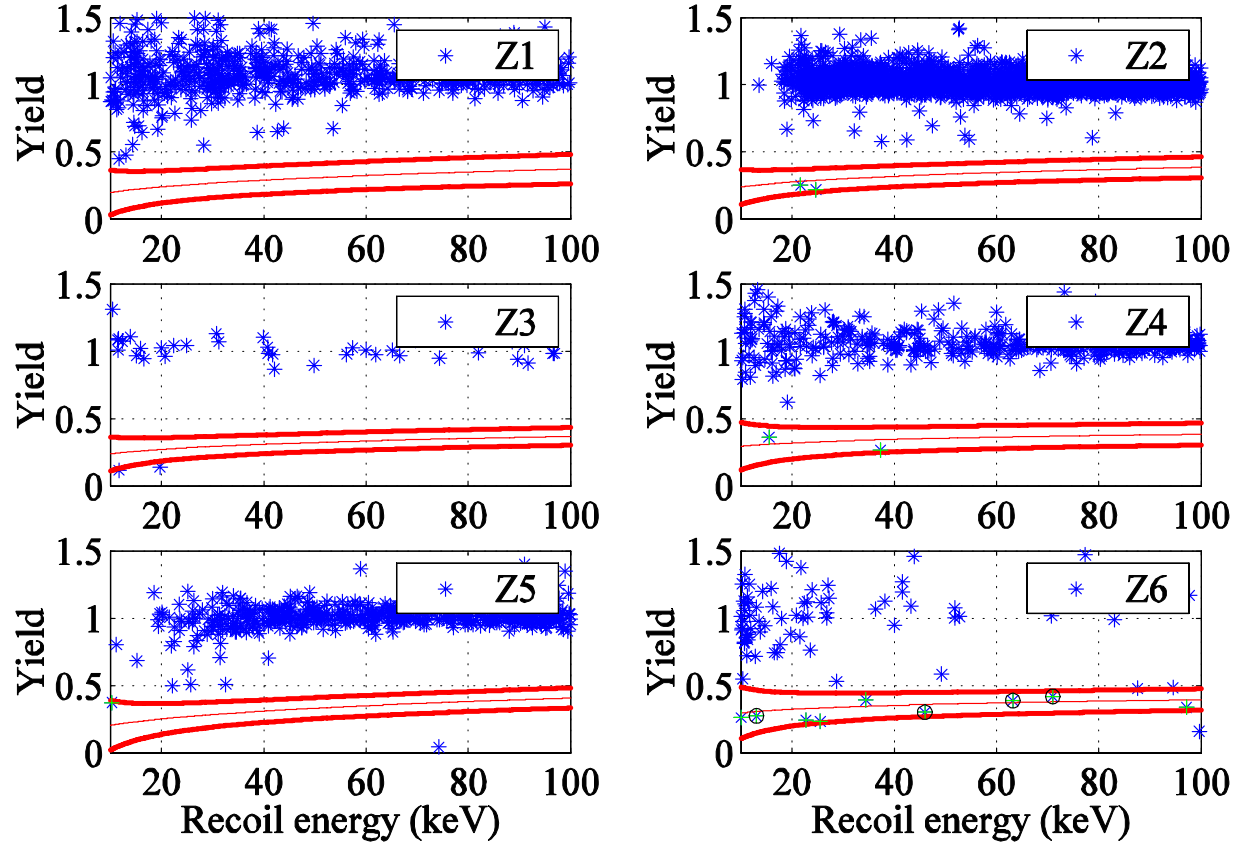

Figure 7.14: Veto anti-coincident events in all six detectors after the application of the rise time cut. The circled events in the signal band indicate the single scatter nuclear recoil events. As can be seen, no single scatter nuclear recoils survive the cut in the germanium detectors. 


\subsubsection{The unblinded analysis}

Following the unblinding of the data, we realized that we had inadvertently used the saturating pulse fitting algorithm for the main analysis instead of using the optimal filter algorithm. The CDMS Analysis package, DarkPipe, uses the F5 algorithm to analyze the data and apply the fits only in the event of saturation of charge pulses. A bug in the code had caused even the unsaturated pulses to be fit by the F5 algorithm. As this is not as optimal a fit as the intended algorithm, the effect of the bug was to cause some events to fail the fiducial-volume cut which in the normal case would have passed the cuts. It also led to a slight broadening of the band after the correction was made.

As a result of redoing the analysis in the non-blind case, we arrived at one event passing all cuts in the single-scatter nuclear-recoil population in the germanium detectors. Figure 7.17 shows the event seen at the edge of the signal region in Z5 with a recoil energy of $64 \mathrm{keV}$. The event seems consistent with out estimate of $0.7 \pm 0.35$ leakage of low-yield surface beta backgrounds in the signal region. The event thus seen seems consistent with our beta leakage events.

\subsection{Limits on the WIMP-nucleon cross section}

Figure 7.18 shows the limits on the WIMP-nucleon cross section set from the first WIMP-search run at Soudan. The solid blue curve sets the limits set from the blind analysis and the dashed blue curve the limits from the unblinded analysis. As can be seen, the limits are a little worse for the second case as we cannot rule out the possibility of the event being a WIMP, even though it is consistent with our estimates of the surface leakage into the signal region for this data set. The Run 118 data sets an upper limit of $4 \times 10^{-43}$ on the WIMP-nucleon cross section at 90\% Confidence Level for a WIMP mass of $60 \mathrm{GeV} / \mathrm{c}^{2}$. The limits are currently the world's most sensitive for WIMP dark matter detection, and are a factor of four below the best previous limits for WIMP masses of $60 \mathrm{GeV} / \mathrm{c}^{2}$ set by the Edelweiss 


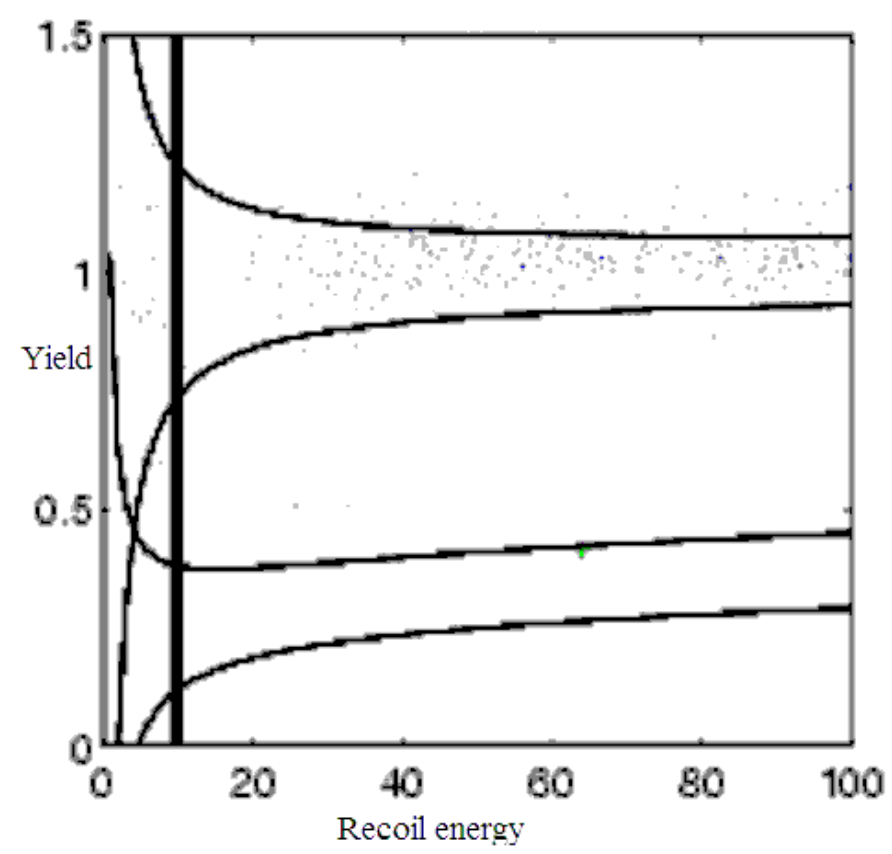

Figure 7.15: The single event passing all cuts in the WIMP-candidate data set in the non-blinded analysis. The event has a recoil energy of $64 \mathrm{keV}$ and seems consistent with the surface leakage estimates for this data set. 
experiment[15]. They are a factor of 8 below the CDMS limit at the shallow site and exclude large regions of WIMP-parameter space. 


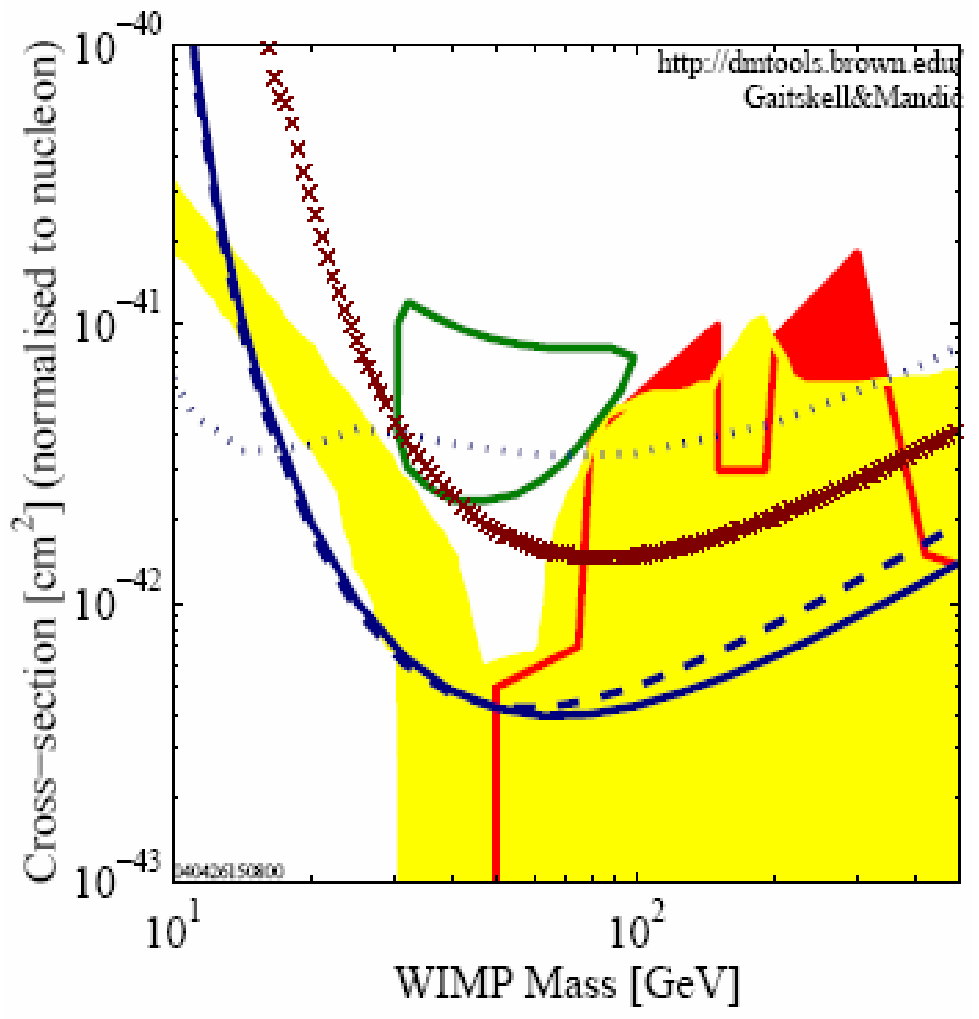

Figure 7.16: Limits on the WIMP-nucleon cross section set from the first WIMPsearch run at Soudan. The solid blue curve sets the limits set from the blind analysis and the dashed blue curve the limits from the unblinded analysis. As can be seen, the limits are a little worse for the second case. The limits set are currently the world's most sensitive for WIMP dark matter detection. The dashed blue line is the limit set by the first analysis of the Run 21 data which includes the statistical subtraction of the neutron background. The brown crosses line are the limits set by Edelweiss. The yellow and red shaded regions represent allowed regions of SUSY parameter space. The green closed circle represents the DAMA $3 \sigma$ allowed region. 


\section{References}

[1] A. da Silva, Ph. D. Thesis, The University of British Columbia, (1996).

[2] R. Nelson, B.S. Dissertation, University of California at Santa Barbara (2003), unpublished.

[3] K. Ruddick, MINOS Internal Note, NuMI-L-210 (1996).

[4] D. Bauer, CDMS Internal Note (2001).

[5] D. S. Akerib et al, astro-ph/0405033 (2004).

[6] S. Yellin, CDMSNote 9704003 (1997).

[7] J. Sander, CDMS Run 118 Ebook note 226 (2004).

[8] T. A. Perera, Ph.D. Thesis, Case Western Reserve University (2002), unpublished.

[9] D. S. Akerib et al, Nucl. Instrum. E3 Meth. in Phys. Res. A, 520, 116 (2004).

[10] V. Mandic, Ph.D. Thesis, University of California at Berkeley (2004), unpublished.

[11] D. Day and L. Baudis, CDMS Run 118 Ebook note 108 (2004).

[12] D. S. Akerib et al, Phys. Rev. D, 68, 82002 (2003).

[13] V. Mandic et al., Nucl. Instr. Meth. A 520, 171 (2004). 
[14] G. Wang and S. Kamat, CDMS Run 118 Ebook note 125 (2004).

[15] A. Benoit et al., Phys. Lett. B 545, 43 (2002). 


\section{Chapter 8}

\section{Improved Sensitivity at a Deeper site}

As the discrimination capabilities of detectors improve, allowing WIMP-searches to extend their sensitivities to WIMP-nucleon cross-sections of $10^{-44}$ and beyond for WIMP masses of $60 \mathrm{GeV} / \mathrm{c}^{2}$, the need for achieving very low background conditions becomes critical. Tagging and isolating neutrons seen at experimental sites is part of such an exercise.

In Chapter IV, I had given a brief summary of the neutron flux seen at the Soudan Underground Facility. In this chapter, I will elaborate on the various contributions to the neutron backgrounds, their relative importance vis-a-vis rate of detection and the simulations that were performed to get a measure of them.

This chapter follows from the discussion of the data analysis of the first Soudan run in Chapter VII and draws inferences from the results of the analysis detailed therein. We use these results to inform our estimates of the muon-coincident and muon-anti-coincident neutron backgrounds seen at the deep site.

The relatively short exposure of Run 118 allowed for a data set that saw no veto-coincident neutrons or possible veto anti-coincident neutrons. We were thus able to set an upper limit on the detection of muon induced neutrons from the 
shield. This upper limit may be used as a cross-check, both on the inferred muon rate and, in turn, on the prediction of the likely number of veto anti-coincident neutrons.

With increasing run time, and a corresponding increase in exposure, we should expect to start seeing a neutron background, from muon interactions both in the shield and rock. The last few sections draw on the results of current analyses to make estimates on the kind of exposure necessary to record neutron events in our detectors.

\subsection{The Neutron Flux at Soudan}

The reduced muon flux translates into a much reduced neutron flux at Soudan. The dominant neutron background here arises from natural radioactivity of the rock and the materials surrounding the detectors. This contribution has been calculated, with $(\alpha, \mathrm{n})$ reactions giving rise to a neutron rate of $(2.1 \pm 0.2) \times 10^{-8}$ neutrons $/ \mathrm{g} / \mathrm{s}$ while spontaneous fission contributes $2.7 \times 10^{-9}$ neutrons/g/s[1].

The incident neutron flux due to these two processes is estimated as 2.0 x $10^{-6}$ neutrons $/ \mathrm{cm}^{2} / \mathrm{s}[2]$. Simulations done while designing the shield indicated that this would yield an interaction rate of $5 \times 10^{-6}$ neutrons $/ \mathrm{kg} / \mathrm{keV} /$ day on the detectors[3], well below the upper limit of $3 \times 10^{-4}$ nuclear-recoil events $/ \mathrm{kg} / \mathrm{keV} /$ day which is the target sensitivity for CDMS II at Soudan.

As explained in Chapter IV, the muon-induced neutron rate at this depth in the rock is more than two orders of magnitude below those arising from natural radioactivity and is estimated as $7.29 \times 10^{-11}$ neutrons/g/s [1].

However, as in the case of the shallow site, the limiting background is expected to arise from very high-energy (energies between $50-600 \mathrm{MeV}$ ) neutrons produced in hadronic cascades that result from inelastic muon-nuclear interactions. Such neutrons are able to punch through the polyethylene and cause scatters in the 
lead that give rise to secondaries that can produce recoils in the energy range of interest.

The following sections discuss the various neutrons fluxes incident on the detectors. Besides simulations, and existing literature on the subject, I have drawn on earlier studies done by Steve Yellin and other members of the CDMS collaboration while designing the shield at Soudan.

\subsubsection{Neutron production from muons at deep sites}

At deep sites, neutron production arises from the direct interaction of the electromagnetic field of the muon with nuclei (primary muon spallation), and the secondaries produced in hadronic cascades generated from this, and from photonuclear interactions of real photons generated from bremsstrahlung, pair production and $\delta$ electrons. Neutrons produced in the first case can have energies of the order of $50-600 \mathrm{MeV}$, sometimes even up to a few $\mathrm{GeV}$. A representative spectrum of these high-energy neutrons is shown later in Figure 8.5. These neutrons have a low interaction rate in the outer polyethylene shield of the detectors, allowing them to punch through this material and cause scatters in the lead, producing secondary neutrons that cause recoils in the energy range of $5-100 \mathrm{keV}$ in the germanium and silicon detectors.

At high energies, the first process is dominated by secondaries in the resulting nuclear showers. The average number of neutrons produced in the nuclear cascade increases with the mean muon energy at that depth, in proportion to $E_{\mu}^{0.75 \pm 0.05}[4]$.

With increasing depth, and hence greater mean muon energy, more neutrons are produced per muon in hadronic cascades. Figures 8.1 and 8.2 illustrate the dependence on depth and the mean muon energy at a site of the contribution of each of these processes to the total neutron yield. 


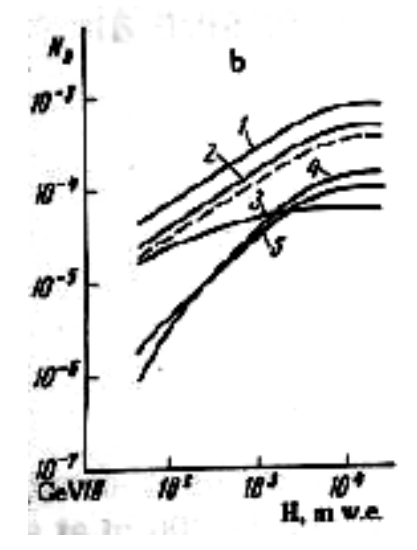

Figure 8.1: Neutron yield per muon per unit path length $\left(\mathrm{g} / \mathrm{cm}^{2}\right)$ of lead as a function of depth: Curve 2) Neutrons produced by virtual photons in hadronic cascades; Curves 3) 5) Neutrons produced by real photons in electromagnetic showers induced by $\delta$ electrons, pair production and bremstrahlung respectively; and Curve 1) Total neutron yield from all processes (taken from [5])

At a mean muon energy of $280 \mathrm{GeV}[6]$, it has been found that $75 \%$ of the neutrons in liquid scintillator arise from hadronic cascades, $20 \%$ from electromagnetic showers and 5\% from the primary muon spallation. This spallation contribution increases to $9 \%$ if secondaries resulting from collision of the primary spallation neutrons are included here.

As the cross-section of electromagnetic muon interactions shows a $\frac{Z^{2}}{A}$ dependence, where $\mathrm{A}$ is the atomic weight of the material, the proportion of neutrons produced in electromagnetic showers increases with heavier target materials.

For lead, at the same depth, the electromagnetic showers rises from $20 \%$ to $42 \%$ while the hadronic cascades give $55 \%$ of the total leaving the remaining $3 \%$ of the neutrons to arise from primary muon spallation processes [6]. Muon capture processes are negligible at depths $>100$ m.w.e [7].

The above-mentioned fractional contributions of the various processes to the total muon-induced neutron production has been drawn from results of FLUKA simulations of muon-induced neutron production in liquid scintillator, lead and 


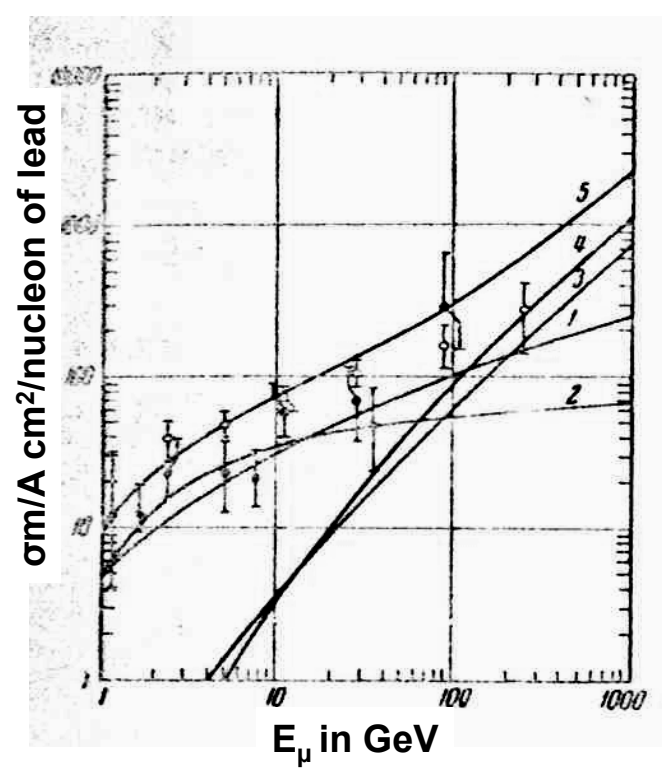

Figure 8.2: Neutron yield per muon per unit path length of lead as a function of depth, and hence mean muon energy: Curve 1) Neutrons produced by virtual photons in hadronic cascades; Curves 2) - 4) Neutrons produced by real photons in electromagnetic showers induced by $\delta$ electrons, pair production and bremstrahlung respectively; and Curve 5) Total neutron yield from all processes. The open circles are experimental data from[22] and the closed circles data from [23] (taken from [16]). 
other materials[6]. We compare these percent contributions in lead with the earlier studies[16] of neutron production from different muon processes at depths corresponding to a mean muon energy of $280 \mathrm{GeV}$. The measurements suggest that the contribution of direct muon interactions is $20 \%$ of the total neutron yield while the rest of the neutrons are produced from pair production, $\delta$ electrons and bremsstrahlung. This discrepancy between the $20 \%$ predicted by analytical studies and measurements of [16] and FLUKA simulations suggest a means of quantifying the systematic uncertainties associated with the predictions of neutron yield in lead at different depths, including that of Soudan.

The primary spallation neutron production spectrum can be considered to have a high-energy (50-600 MeV) and a low-energy (<50 MeV) component. The high-energy component can be represented by the parametrization[8]:

$$
\frac{d N}{d E}=\left\{\begin{array}{c}
6.05 e^{\left(-\frac{E}{77}\right)} \text { for } 50<\mathrm{E}<200 \mathrm{MeV} \\
e^{\frac{-E}{250}} \text { for } \mathrm{E}>200 \mathrm{MeV}
\end{array}\right\}
$$

where $\mathrm{E}=$ neutron energy in $\mathrm{MeV}$. The spectrum does not depend on the nature or energy of the initial projectile provided the latter has energy $>2 \mathrm{GeV}[9]$.

An energy spectrum of neutrons produced by a FLUKA simulation of the propagation of muons in $1 \mathrm{~cm}$ of lead at a depth of $2450 \mathrm{~m}$.w.e shows good agreement with this parametrization[10]. In the simulation, the neutrons produced are the primary spallation neutrons as nuclear showers are yet to develop in such a small thickness of lead.

At low energies, muon-nuclear interactions proceed through the giant dipole resonance (described in Chapter IV) yielding neutrons with the evaporation spectrum used to describe muon capture processes at low depths. The lower energy component of the primary spallation spectrum can thus be given by the functional form[11]: 


$$
\frac{d N}{d E}=\left\{\begin{array}{c}
0.812 E^{5 / 11} e^{\left(-\frac{E}{1.22}\right)} \text { for } \mathrm{E}<4.5 \mathrm{MeV} \\
0.018 e^{\frac{-E}{9}} \text { for } 50 \mathrm{MeV}>\mathrm{E}>4.5 \mathrm{MeV}
\end{array}\right\}
$$

where $\mathrm{E}$ is the neutron energy in $\mathrm{MeV}$. The same FLUKA simulation described above produces a low-energy muon induced neutron spectrum that agrees with this functional form[10].

Neutrons arising from $(\gamma, n)$ reactions involving real photons in electromagnetic showers have relatively smaller energies, going up to $\sim 50 \mathrm{MeV}$. As the spectrum of shower photons falls off as $\frac{1}{E_{\gamma}^{2}}[12]$, neutrons in these showers are generated mainly by low-energy photons. These neutrons follow the evaporative spectrum, represented by Equation 8.2., by the time they reach the inner cans of the icebox containing the detectors.

The high-energy primary neutrons generate secondaries that eventually produce a spectrum similar to the evaporative spectrum shown in Equation 8-2.

To estimate and cross-check the neutron production rate, we make use of the current understanding of the yield, both from simulations and direct measurements. The measurements used include upper limits set from the results of the data analysis of the muon coincident and anti-coincident nuclear-recoil event populations seen in Run 118 at Soudan. Various studies of muon induced neutron production at different depths have made the assumption that neutrons are produced by muons, all having the energy equal to the mean muon energy at that depth. FLUKA[13] simulations of the total neutron yield per muon per unit path length in a material for a beam of mono-energetic muons incident on liquid scintillator indicate a dependence [14] on muon flux given by:

$$
N_{\mu}=4.14 \times\left(E_{\mu}^{0.74}\right) \times 10^{-6} \mathrm{n} / \mu / \mathrm{g}^{-\mathrm{cm}^{-2}} .
$$

Here $E_{\mu}$ may be taken as the mean muon energy. Neutron production from the differential muon energy spectra has been estimated[4] to be smaller by $10-15 \%$ 
for depths from surface to $3 \mathrm{~km}$.w.e. below and for mean muon energies between 100 - $300 \mathrm{GeV}$ compared with considering an incident muon spectrum containing muons, all of which have energies equal to the mean muon energy at that depth.

In the following sections, I will look first at the muon induced neutron background in the material of the shield, and subsequently, at such neutron production in the rock of the experimental cavern.

\subsection{Neutrons produced in the shield}

Muon-induced interactions in the lead, copper and polyethylene layers of the shield can give rise to neutrons. Though vetoed out of the final dark matter analysis, their study can serve as an important measure of the neutron production processes in different materials at this depth.

The main contributors are the $18 \mathrm{~cm}$ of outer lead and the $4.5 \mathrm{~cm}$ of ancient lead. A large number of the low-energy neutrons produced in the lead are moderated by the $7.6 \mathrm{~cm}$ of inner polyethylene.

Neutrons produced in the copper of the tower housing and icebox cans also add to the flux, though this neutron yield is expected to be substantially lower, owing to the smaller thickness and lower atomic weight of the material.

The GEANT3[15] routines used start from the neutron flux and do not simulate the muon-induced neutron production. The production rate of neutrons from muon-induced processes in lead and other materials at deep sites has been studied in detail in recent times, specifically in the context of the impact of this background on dark matter searches. I will draw on this vast body of literature to base my conclusions primarily with regard to the appropriate muon-induced neutron yield.

There have been several measurements of the muon induced neutron production in lead at different depths. The average neutron production per nucleon in 
lead has been measured [16][17][18] as $\frac{m \sigma}{A}=(290 \pm 50) \times 10^{-29} \mathrm{~cm}^{2} /$ nucleon for lead where $\mathrm{m}=$ multiplicity, $\mathrm{A}=$ atomic weight and $\sigma$ is the cross-section for a depth of 800 m.w.e. where the mean muon energy is $110 \mathrm{GeV}$. The total neutron yield in lead at $110 \mathrm{GeV}$ is thus $(1.8 \pm 0.3) \times 10^{-3}$ neutrons $/ \mathrm{muon} /\left(\mathrm{g} / \mathrm{cm}^{2}\right)$.

Both FLUKA simulations and direct measurements at different depths have affirmed the $\mathrm{E}_{\mu}^{0.75 \pm 0.05}$ dependence[4] of the neutron production per muon per unit path length of material on the mean muon energy at a site. Assuming this $\mathrm{E}_{\mu}^{0.75 \pm 0.05}$ dependence, and noting that the mean energy at the Soudan depth is $210 \mathrm{GeV}[1]$, we arrive at $(2.9 \pm 0.5) \times 10^{-3}$ neutrons $/ \mu /\left(\mathrm{g} / \mathrm{cm}^{2}\right)$ of lead for the Soudan depth.

The corresponding value for copper at Soudan has been estimated from [16][20] as $(8.3 \pm 0.9) \times 10^{-4}$ neutrons $/ \mu /\left(\mathrm{g} / \mathrm{cm}^{2}\right)$.

As noted earlier, the contribution of neutrons produced in hadronic cascades to the total yield depends both on the target material and the depth, or the mean muon energy of the muon inducing these processes. To estimate the contribution of the hadronic interactions (the high-energy component) to the total neutron yield, I will use two alternate methods based on direct measurements and simulations.

In the first case, I will use the relation of mean muon energy with the total neutron yield as given in [16]. The dependence is arrived at both through analytical calculations and measurements of the total yield in lead at different depths for a detector having a lead thickness of $20 \mathrm{~cm}$, comparable to the $22.5 \mathrm{~cm}$ of lead used in our shield geometry. The comparable thickness of material allows us to assume a similar development in secondary cascades though we will be extrapolating from the case of $\mathrm{E}_{\mu}=110 \mathrm{GeV}$ to the Soudan equivalent of $210 \mathrm{GeV}$.

The direct muon interaction contribution cited includes both the muon spallation mechanism and that from the hadronic cascades. We make use of the literature to estimate the contribution of each of these processes to the total yield from hadronic interactions, the details of which are given in the subsequent paragraphs. In the second case, we use results from FLUKA simulations for total 
neutron yield in lead from the various muon induced processes. The simulations quantify the contribution of the direct muon spallation, the hadronic cascades and the electromagnetic showers to the yield.

At the depth of Soudan (2090 m.w.e.), the first method predicts $25 \%$ of the total neutron yield in lead to arise from direct muon interactions while those arising from $\delta$ electron production, bremsstrahlung and pair production in electromagnetic showers make up the rest[16]. This is inferred from Figures 8.1 and 8.2 where the yield variation with depth and muon energy respectively is shown.

The $25 \%$ contribution from direct interactions due to virtual photons includes both the primary muon spallation process as well as the secondary cascades that arise from it. Assuming this to be the production rate for the high-energy component of the neutron spectrum produced in lead is an overestimate as the secondaries include neutrons of energies $<50 \mathrm{MeV}$. We, therefore, use this as a means of getting an upper bound on our predictions.

Using the second method, we can set lower bounds on our predictions of the veto-coincident rate by using results from the simulations of the neutron yield in lead. The simulations indicate that the primary spallation mechanism contributes $5 \%$ of the total neutron yield in lead at depths comparable to ours[10]. This compares well with $3 \%$ of neutrons from primary spallation at higher mean muon energies as indicated by FLUKA and other simulations for other deep sites [6][19], as was noted in Section 8.1. Experimental observations also affirm that the contribution of the cascades increases with increasing depth, causing neutrons arising from the primary spallation process to have a reduced contribution[9].

Assuming a 5\% contribution to the total yield from FLUKA simulations, the high-energy component can be estimated as $1.4 \times 10^{-4}$ per muon per $\left(\mathrm{g} / \mathrm{cm}^{2}\right)$ for lead and $4.1 \times 10^{-5}$ per muon per $\left(\mathrm{g} / \mathrm{cm}^{2}\right)$ for copper. This component can be represented by Equation 8.1 which, at energies $>200 \mathrm{MeV}$, agrees well with the $e^{\frac{-E}{230 \mathrm{MeV}}}$ dependency of these neutrons arrived at by an alternate study at Soudan[1].

To get an upper bound on the systematic uncertainties involved, we consider 
the case wherein the contribution from direct interactions is entirely made of the high-energy component. The yield is then estimated as $(7.2 \pm 1.9) \times 10^{-4}$ neutrons per muon $/\left(\mathrm{g} / \mathrm{cm}^{2}\right)$ for lead and $(3.3 \pm 0.3) \times 10^{-4}$ neutrons per muon $/\left(\mathrm{g} / \mathrm{cm}^{2}\right)$ for copper. However, as noted earlier, this includes contribution from cascade neutrons which contain a low-energy component. We integrate the spectra described by Equation 8.1 and Equation 8.2 from $0.1-600 \mathrm{MeV}$ and determine that the highenergy component represents $58 \%$ of the contribution from direct muon interactions as specified earlier. That is, the appropriate neutron yield from lead for the highenergy component is $58 \%$ of the $25 \%$ of the total muon-induced neutron yield.

The final value of the yield of the high-energy component by combining the two methods and using the discrepancy to establish a systematic error is found to be $(2.8 \pm 1.4) \times 10^{-4}$ neutrons $/ \mu /\left(\mathrm{g} / \mathrm{cm}^{2}\right)$ for lead and $(1.2 \pm 0.7) \times 10^{-4}$ neutrons $/ \mu /\left(\mathrm{g} / \mathrm{cm}^{2}\right)$ for copper.

The lower energy neutrons assume the functional form given by Equation 8.2. The neutron yield, after folding in the systematic errors in the same way as the above case, is $(2.6 \pm 0.5) \times 10^{-3}$ neutrons $/ \mu /\left(\mathrm{g} / \mathrm{cm}^{2}\right)$ for lead and $(7.1 \pm 1.1)$ $\mathrm{x} 10^{-4}$ neutrons $/ \mu /\left(\mathrm{g} / \mathrm{cm}^{2}\right)$ for copper.

GEANT3 simulations of these two components with the above-mentioned normalizations were run with the full Run 118 detector and shielding assembly in place. The results of the simulations are described in the subsequent sections. The event rate on the detectors in the energy range of $10-100 \mathrm{keV}$ was obtained. The recoil spectra for single-scatter and multiple-scatter events in the detectors were also recorded.

The predictions of the simulations were then compared against muon coincident single and multiple-scatter events in the nuclear-recoil band. This allows us to compare the accuracy of the rates, and expected number of events from the simulations, with what was seen in the data.

The number predicted in the simulations were found to be $1.94 \pm 0.44$. Within the systematic uncertainties, they were well within the upper limit of 2.3 
events at 90\% CL set by the observation of zero muon-coincident nuclear-recoil events in the data run.

The muon induced neutrons in the shield are vetoed and do not feature in the WIMP-search. They however provide a means of setting an upper limit on the number of "external" neutrons seen in a data run. As explained earlier, these high-energy neutrons are produced in the rock and are able to enter the shielding assembly without detection. They scatter inside the shield, yielding secondaries that mimic WIMPs.

The $\mathrm{E}_{\mu}^{0.75}$ dependence of both neutron populations on mean muon energy provided a means of using the data and simulations output of the shield neutrons to make predictions on the rate of the veto-anti-coincident neutron population. The procedure is explained in Section 8.8.

The simulations also provide a means of making conservative estimates of the vetoed and un-vetoed neutron events in Run 118 and for subsequent longer exposures. Based on the simulation of the internal neutrons and the upper limit on the veto-coincident rate as obtained from the data, an upper limit on the expected number of punch through neutrons seen at Soudan is detailed in Section 8.8.

\subsection{Neutrons produced in the rock}

As before, we can consider the neutron production in the rock to be made of the high-energy component arising from hadronic cascades and neutrons of lower energies from electromagnetic showers. The almost $50 \mathrm{~cm}$ of polyethylene between this external flux and the detectors moderate all but the high $(50-600 \mathrm{MeV})$ energy component. These neutrons have interaction lengths of $\sim 100 \mathrm{~cm}$ in the polyethylene allowing them to punch through the outer polyethylene with ease to produce secondaries in the shield that produce recoils of energies similar to the expected WIMP signal. As was the case at SUF, this is expected to be our limiting external background. 
Equation 8.1 represents the energy spectrum of these punch through neutrons. The neutron yield in rock at Soudan has been measured as $(3.3 \pm 1.0) \mathrm{x}$ $10^{-4}$ neutrons $/ \mu /\left(\mathrm{g} / \mathrm{cm}^{2}\right)[1]$. Extrapolating from the $3.56 \times 10^{-4}$ neutrons $/ \mathrm{muon} /\left(\mathrm{g} / \mathrm{cm}^{2}\right)$ from FLUKA simulations[19] of the neutron yield in rock at a mean muon energy of $270 \mathrm{GeV}$ using the $\mathrm{E}_{\mu}^{0.75}$ dependence gives us $2.95 \times 10^{-4}$ neutrons $/ \mu /\left(\mathrm{g} / \mathrm{cm}^{2}\right)$ at the Soudan energy of $210 \mathrm{GeV}$ which is well within the uncertainties quoted above.

Using the functional form for yield from Equation 8.3 for liquid scintillator at $210 \mathrm{GeV}$ mean muon energy and folding in the $\mathrm{A}^{0.76 \pm 0.01}[6]$ dependency on the atomic weight of rock yields $3.94 \times 10^{-4}$ neutrons $/ \mu /\left(\mathrm{g} / \mathrm{cm}^{2}\right)$, again within the uncertainties quoted above.

The yield quoted above is from all muon-induced processes in the rock. As explained earlier, the contribution from the electromagnetic showers as well as the low-energy neutrons from the hadronic cascades have energies $<50 \mathrm{MeV}$ and are well moderated by the $40 \mathrm{cms}$ of outer polyethylene. It is the very high-energy (50 - $600 \mathrm{MeV}$ ) component of the cascade that punches through the polyethylene to generate secondaries that mimic the WIMP signal.

At Soudan depth, there are almost equal contributions from the hadronic and electromagnetic showers [12]. We use the measured neutron yield of (3.3 1.0$)$ x $10^{-4}$ neutrons $/ \mu /\left(\mathrm{g} / \mathrm{cm}^{2}\right)$ for high-energy neutrons from the Soudan rock.

Two varying measurements exist for the muon flux at the site. The flux has been measured as $1.8 \times 10^{-3} / \mathrm{m}^{2} / \mathrm{s}$ by the Soudan II detector studies at the same site and as $(2.21 \pm 0.03) \times 10^{-3} / \mathrm{m}^{2} / \mathrm{s}[20]$ using a muon telescope by our experiment. For our purposes, we interpret this discrepancy as a systematic error and take the value of $(2.0 \pm 0.2) \times 10^{-3} / \mathrm{m}^{2} / \mathrm{s}$ for the muon flux. The production rate is determined as $(6.6 \pm 2.1) \times 10^{-8}$ neutrons $/ \mathrm{kg} / \mathrm{s}$ on the production rate of neutrons from the rock.

Simulations using GEANT3 were run assuming the production rate in rock as specified and the high-energy spectrum as described by Equation 8.1. The neutrons were generated in a 10 meter shell of rock and the emergent flux and spectrum 
were used as the inputs to the Run 118 simulation. This emergent flux and spectrum were thrown from the surface of a $220 \times 220 \times 220 \mathrm{~cm}$ cube surrounding the outer shielding assembly. The neutrons were allowed to propagate through the configuration of the shield and the event rate in the detectors was recorded.

The expected number of external neutrons seen in Run 118 was (0.052 \pm 0.024$)$. An estimated exposure of $500 \mathrm{~kg}$-days is needed to start seeing neutron events due to the external neutrons from the rock.

\subsection{Simulating the vetoed neutron population}

In this section, I will examine the results of the simulations of the muon induced neutrons in the material of the shield. As explained earlier, neutrons from the lead dominate this population. Neutrons from the copper of the icebox are also considered as, unlike the lead, no polyethylene shields the detectors from these neutrons. The total neutron yield, based on literature, has been estimated as $(2.9 \pm 0.5)$ x $10^{-3}$ neutrons $/ \mu /\left(\mathrm{g} / \mathrm{cm}^{2}\right)$ for lead and $(8.3 \pm 0.9) \times 10^{-4}$ neutrons $/ \mu /\left(\mathrm{g} / \mathrm{cm}^{2}\right)$ for copper, as described in Section 8.2.

The term "internal" neutrons is used to refer to this neutron population vis-a-vis "externals" for the veto-anti-coincident neutrons produced from the rock. About 40\%[24] of the so-called external neutrons trigger the veto and thus populate the internal neutron distribution in the data. This may lead to an overestimate at the $5-10 \%$ level in the predictions of the internal neutrons based on the analysis outlined earlier.

To simulate the high-energy component, neutrons were thrown from the lead and copper of the shield assuming a functional form given by Equation 8.1 and a yield of $(2.8 \pm 1.4) \times 10^{-4}$ neutrons $/ \mu /\left(\mathrm{g} / \mathrm{cm}^{2}\right)$ for lead. For copper the yield was taken as $(1.2 \pm 0.7) \times 10^{-4}$ neutrons $/ \mu /\left(\mathrm{g} / \mathrm{cm}^{2}\right)$. Using the value of the muon flux at Soudan as specified earlier, we get a production rate of $(6.2 \pm 3.1) \times 10^{-8}$ 
neutrons $/ \mathrm{kg} / \mathrm{s}$ in lead. For copper, the corresponding production rate was $(2.6 \pm 1.5)$ x $10^{-8}$ neutrons $/ \mathrm{kg} / \mathrm{s}$.

The event rates were determined, first in raw live days and then with the data efficiencies folded in. An estimate was made of the expected number of vetocoincident neutrons seen for the Run 118 exposure based on this analysis. The results are presented in Table 8.1

To simulate the low-energy component, neutrons were thrown from the lead and copper of the shield assuming the functional form given by Equation 8.2 and a yield of $(2.6 \pm 0.5) \times 10^{-3}$ neutrons $/ \mu /\left(\mathrm{g} / \mathrm{cm}^{2}\right)$ for lead. For copper, the value was $(7.1 \pm 1.1) \times 10^{-4}$ neutrons $/ \mu /\left(\mathrm{g} / \mathrm{cm}^{2}\right)$. The production rate is similarly estimated as $(5.7 \pm 1.1) \times 10^{-7}$ neutrons $/ \mathrm{kg} / \mathrm{s}$ in lead. The value was taken as $(1.6 \pm 0.2) \times 10^{-7}$ neutrons $/ \mathrm{kg} / \mathrm{s}$ for copper.

As before, the event rates were determined, both with and without the efficiencies folded in. An estimate was made of the likely number of neutrons in Run 118 due to the low-energy component of the input neutron flux from the shield. Table 8.1 summarizes the event rates for single-scatter events for the four germanium and two silicon detectors for both energy contributions based on Monte Carlo simulations of these neutrons in the material of the shielding. The energy range is $10-100 \mathrm{keV}$ for ZIP detectors Z2, Z3, Z5 and Z6 and $20-100 \mathrm{keV}$ for detectors $\mathrm{Z} 1$ and Z4. Table 8.2 gives the expected number of neutrons in the entire Run 118 data set based on the simulations and the efficiencies of the data cuts as applied to the simulations.

The simulations predict $1.94 \pm 0.44$ single-scatter neutron events in the four germanium detectors and $0.89 \pm 0.18$ such events in the two silicon detectors for the exposure of Run 118. This is with the efficiencies of the data cuts folded in. As we will see in the subsequent section, this predicted rate is consistent with the upper limit set by results of the data analysis of the muon coincident nuclear-recoil events. 


\begin{tabular}{|l|l|l|l|l|}
\hline $\begin{array}{l}\text { Rates } \\
(\text { cts/kg/day }\end{array}$ & $\begin{array}{l}\text { Coadded } \\
\text { Ge (no } \\
\text { effcs) }\end{array}$ & $\begin{array}{l}\text { Coadded } \\
\text { Ge (effcs) }\end{array}$ & $\begin{array}{l}\text { Coadded } \\
\mathrm{Si} \\
\text { effcs })\end{array}$ & $\begin{array}{l}\text { Coadded } \\
\text { Si (effcs) }\end{array}$ \\
\hline $\begin{array}{l}50<\mathrm{E}< \\
600 \mathrm{MeV}\end{array}$ & $\begin{array}{l}(3.6 \pm 1.8) \\
\mathrm{x} 10^{-2}\end{array}$ & $\begin{array}{l}(1.4 \pm 0.7) \\
\mathrm{x} 10^{-2}\end{array}$ & $\begin{array}{l}(7.6 \pm 3.8) \\
\mathrm{x} 10^{-2}\end{array}$ & $\begin{array}{l}(2.5 \pm 1.2) \\
\mathrm{x} 10^{-2}\end{array}$ \\
\hline $\begin{array}{l}\mathrm{E}<50 \\
\mathrm{MeV}\end{array}$ & $\begin{array}{l}(5.5 \pm 0.1) \\
\mathrm{x} 10^{-2}\end{array}$ & $\begin{array}{l}(2.2 \pm 0.4) \\
\mathrm{x} 10^{-2}\end{array}$ & $\begin{array}{l}(1.6 \pm 0.3) \\
\mathrm{x} 10^{-1}\end{array}$ & $\begin{array}{l}(5.9 \pm 1.1) \\
\mathrm{x} 10^{-2}\end{array}$ \\
\hline
\end{tabular}

Table 8.1: Event rates of the internal neutrons based on Monte Carlo simulations assuming the spectral representations in Equation 8.1 and Equation 8.2 for the high energy and low energy component of the neutron production in the shield. The rates are in events $/ \mathrm{kg} /$ day in the energy range of $10-100 \mathrm{keV}$ (see text for details). The expected number of neutron candidate events in the four germanium and two silicon detectors for the duration of Run 118 with efficiencies folded in is given in the last row.

\begin{tabular}{|c|c|c|}
\hline Expected Number of neutrons & Ge detectors & Si detectors \\
\hline $50 \mathrm{MeV}<\mathrm{E}<600 \mathrm{MeV}$ & $0.77 \pm 0.38$ & $0.27 \pm 0.13$ \\
\hline $\mathrm{E}<50 \mathrm{MeV}$ & $1.17 \pm 0.22$ & $0.62 \pm 0.12$ \\
\hline Total & $1.94 \pm 0.44$ & $0.89 \pm 0.18$ \\
\hline
\end{tabular}

Table 8.2: Expected number of neutrons based on Monte Carlo simulations assuming the spectral representations in Equation 8.1 and Equation 8.2 for the high energy and low energy component of the neutron production in the shield. The expected number of neutron candidate events in the four germanium and two silicon detectors for the duration of Run 118 with all efficiencies folded in is given. 


\subsection{Analysis of veto-coincident nuclear-recoil events in the data}

The veto coincident nuclear-recoil events data set of Run 118 was studied for possible single and multiple events that were likely neutrons produced in the shield. The analysis applied the following cuts (explained in detail in Chapter VII) :

- Data cleaning cuts to remove data sets with trigger bursts, excess noise and events without global trigger.

- Charge and phonon threshold cuts.

- A nuclear-recoil band cut selecting events in the signal region.

- A fiducial-volume cut selecting events consistent with noise at $3 \sigma$ in the outer charge-electrode region. The upper bound of the band selected was modified to exclude noise events in the lower energy region and include real events in the inner electrode region at higher energies.

- A veto cut to include events consistent with a hit in the scintillator muon veto, i.e., where the time to the most recent veto hit is $<50$ us.

- A singles cut that defines events as single-scatter events if only one detector has a phonon signal larger than $6 \sigma$ of the noise.

- A multiples cut that defined events as multiple-scatter events if all detectors pass the cleaning cuts and at least two detectors also pass the fiducial-volume cut.

Figures 8.3 and 8.4 show the muon-coincident nuclear-recoil events seen in the low background data set for Run 118. The first figure is without the rise time cut applied and the second applies the rise time cut. 


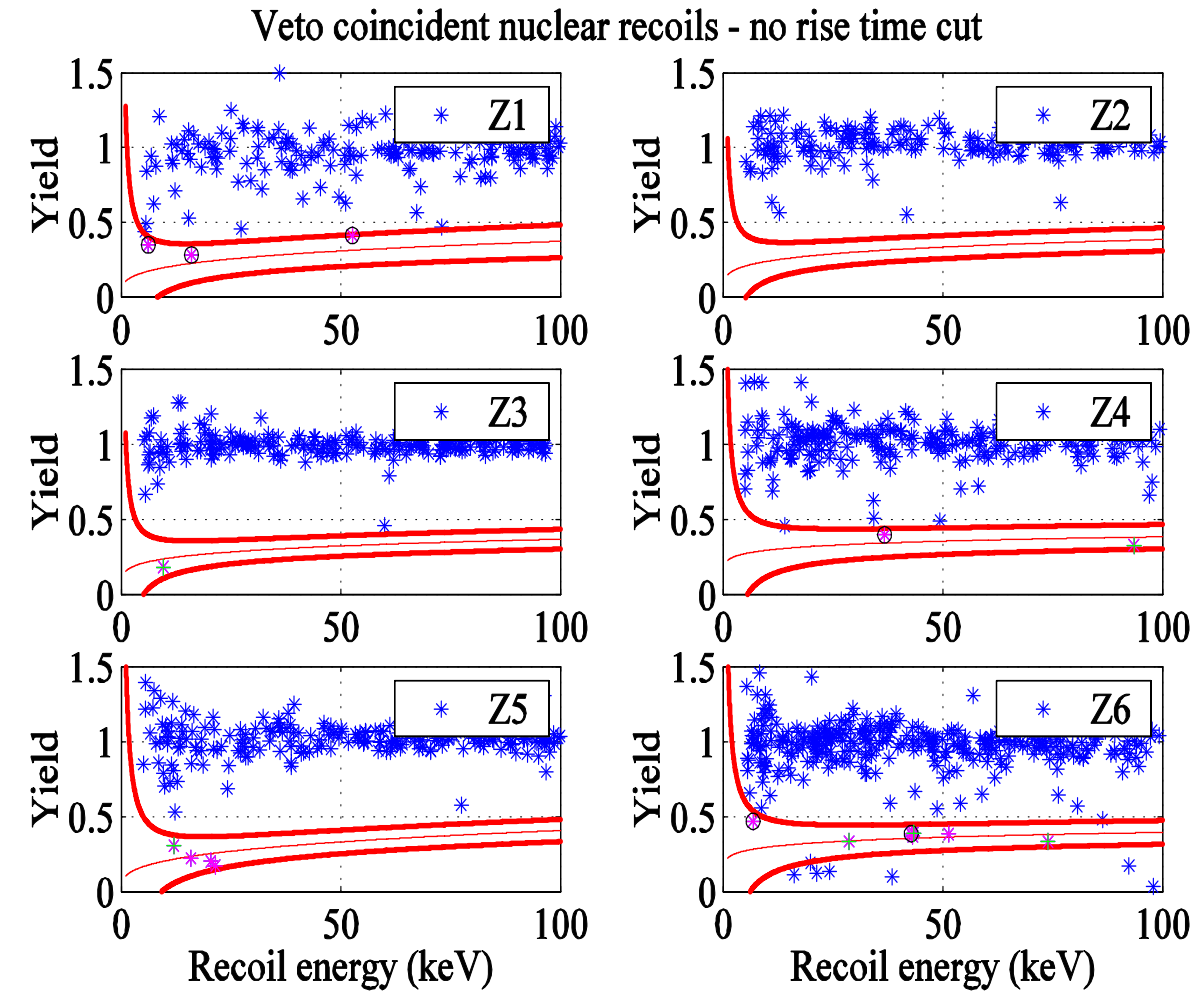

Figure 8.3: Veto coincident nuclear recoil events in the six detectors for the entire low background data set. The signal region is defined within the nuclear recoil bands as shown. The blue crosses are events passing cleaning and fiducial volume cuts. The circled events are single scatter nuclear recoil events and the crosses are multiple scatters in the signal region. No rise cut has been applied 

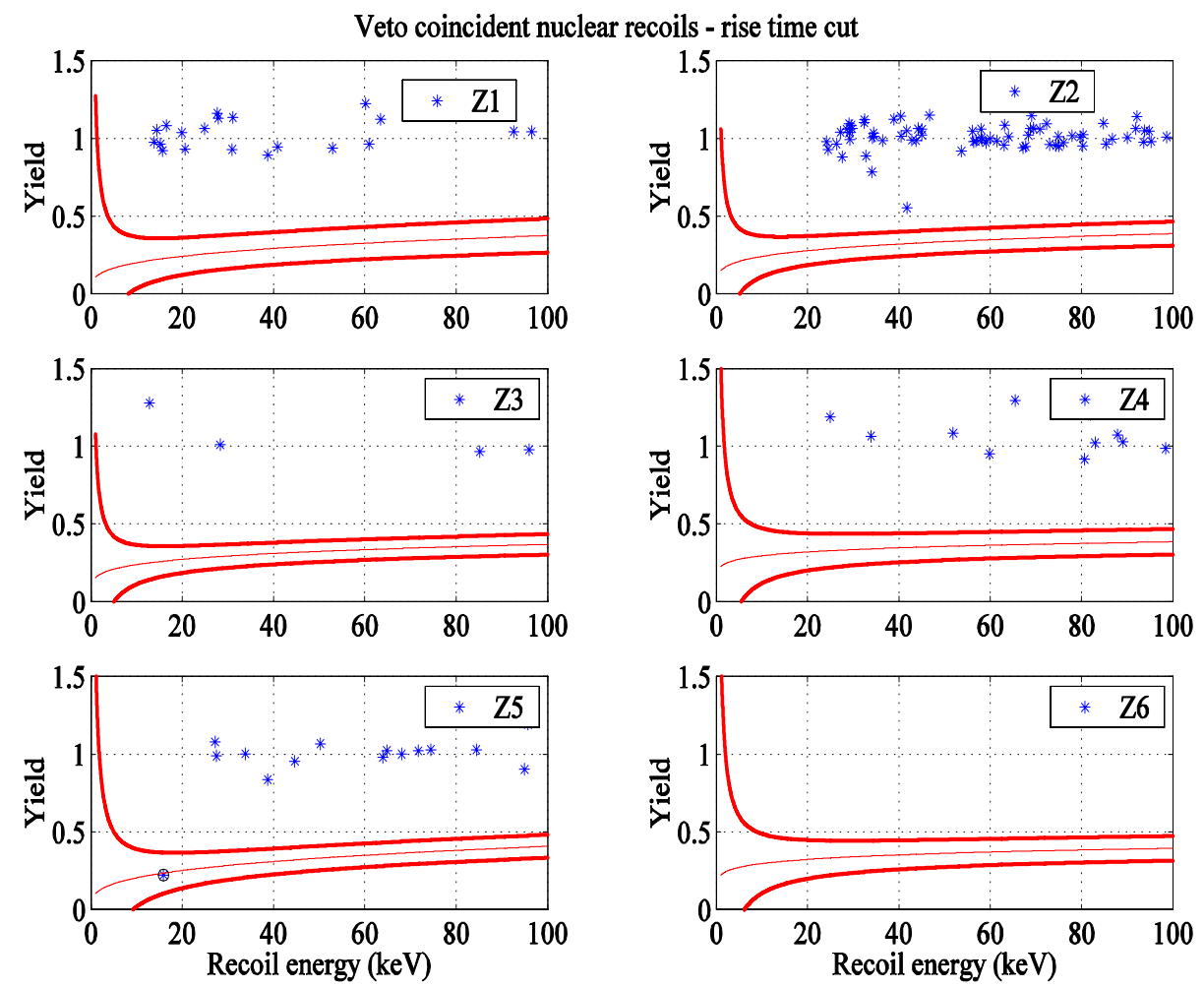

Figure 8.4: Veto coincident nuclear recoil events in the six detectors for the entire low background data set. The signal region is defined within the nuclear recoil bands as shown. The circled event in Z5 is the only event passing the rise time cut. It is a multiple scatter in Z5 and Z6 that fails the fiducial volume cut in Z6 and so fails the multiples cut. A rise cut has been applied 


\begin{tabular}{|c|c|}
\hline Data & Simulations \\
\hline$<2.3$ @ 90\% CL & $1.94 \pm 0.44(\mathrm{sys})$ \\
\hline
\end{tabular}

Table 8.3: Comparison of data and simulations for the veto-coincident nuclear recoil events.

Only one event in ZIP5 survived the rise time cut selecting events consistent with neutrons and rejecting events with low-yield like surface betas. The event was a multiple-scatter event in ZIP5 and ZIP6 which failed the fiducial-volume cut in ZIP6. It left a signature in the muon veto that was consistent with a gamma - lower energy deposition and just one of the scintillator paddles was triggered - raising the possibility that this might not be a true veto coincident neutron but more likely a surface beta that arrived in coincidence with a gamma triggering the veto.

The likelihood of an external neutron passing through the veto in coincidence with a gamma particle can also be discounted based on the time between the occurrence of the event and a trigger in the veto. The timing information discounts the likelihood $\theta$ of a prompt occurrence of an external neutron recoil in the detector in accidental coincidence with a gamma triggering the veto.

We, therefore, conclude that there were no single or multiple veto-coincident events that appeared likely neutrons in the low background data of Run 118. We set an upper limit of 2.3 events at $90 \%$ confidence level for this data set.

\subsubsection{Veto coincident neutrons - comparing data and sim- ulations.}

Here is a comparison of the data and simulations for the different neutron populations. The absence of likely neutrons in the data is compared with the expected number of such events from the Monte Carlo simulations of the propagation of muon-induced neutrons in the shield. Table 8.3 shows the comparisons. 
The absence of neutron candidates in the muon coincident nuclear-recoil event data allows us to set an upper limit of seeing 2.3 events at the $90 \% \mathrm{CL}$. The simulations predict $1.94 \pm 0.44$ events in the four germanium detectors. A Poisson distribution centered at 1.94 fluctuates to zero events $15 \%$ of the time. The expected number of events as predicted by simulations are therefore reasonably consistent with the non-observance of events in the data.

This prediction is based on production rates derived from direct measurements and analytical calculations taken from literature. Where it was possible, we included the known systematic uncertainties associated with them, as stated above. Such uncertainties will be better quantified when the neutron spectrum is generated from the simulation of the incident muon flux as is planned for the next stage of the CDMS experiment.

\subsection{Simulating the un-vetoed neutron population}

From the yield of $(3.3 \pm 1.0) \times 10^{-4}$ neutrons $/ \mu /\left(\mathrm{g} / \mathrm{cm}^{2}\right)$ in rock at Soudan and the measured muon flux of $(2.0 \pm 0.2) \times 10^{-3} / \mathrm{m}^{2} / \mathrm{s}$, we get a production rate of $(6.6 \pm 2.1) \times 10^{-8}$ neutrons $/ \mathrm{kg} / \mathrm{s}$. These high-energy neutrons were generated using the functional form in Equation 8.1 and allowed to propagate through a 10 meter shell of rock. The emergent spectrum and flux were used as the input neutron flux to the Run 118 geometry. The flux emerging from the rock surface was found be $(2.9 \pm 0.9) \times 10^{-9}$ neutrons $/ \mathrm{cm}^{2} / \mathrm{s}$. The high-energy neutron flux emerging from the rock is shown in Figure 8.5.

The spectrum was incident on a cube of sides of length $220 \mathrm{~cm}$ external to the shielding assembly. The neutron rate incident on the detectors was $(8.4 \pm 2.6) \mathrm{x}$ $10^{-4}$ neutrons/s. Using GEANT3, the neutrons were propagated in the shield and the event rate in the detectors recorded. Figure 8.6 shows the recoil spectra for the detectors due to the external neutron flux. 
High energy neutrons from the rock

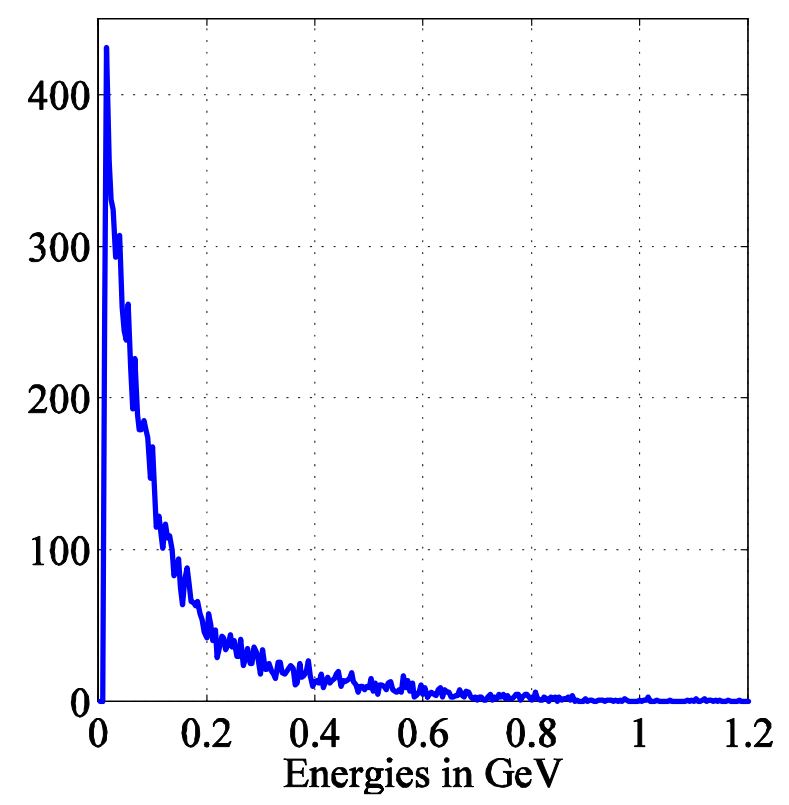

Figure 8.5: Flux of the external neutrons emerging from the rock and entering the experimental cavern. 

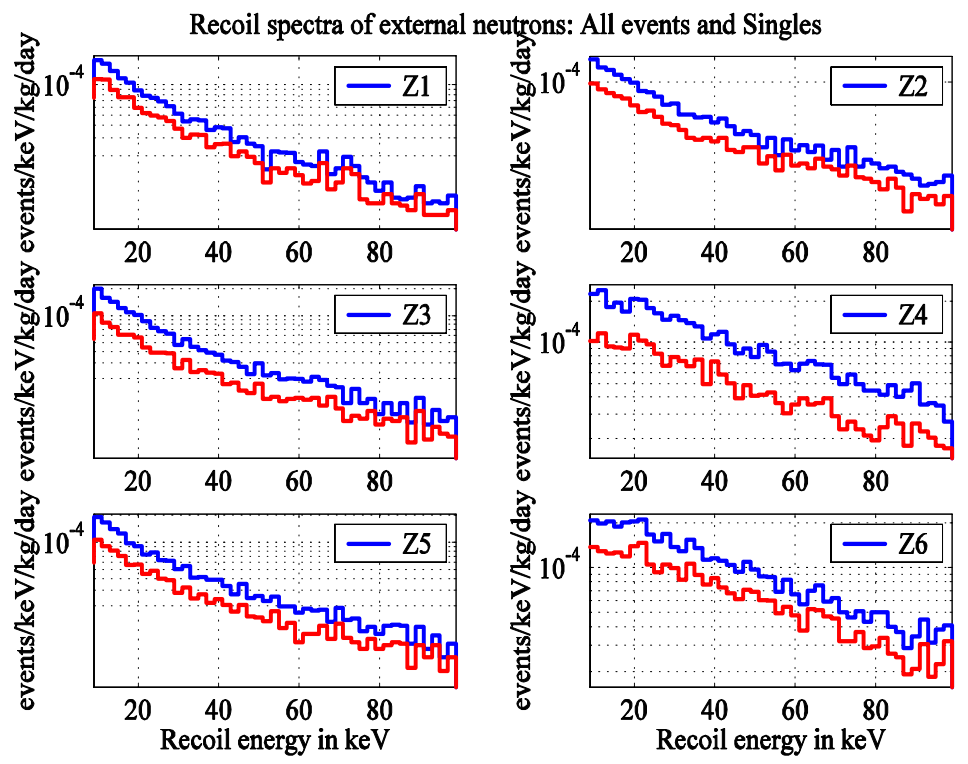

Figure 8.6: Recoil spectra for all events and single scatters (upper and lower curves respectively) from the external neutron flux.

Table 8.4 summarizes the event rates for single-scatter events for the four germanium and two silicon detectors based on Monte Carlo simulations of the external neutrons. The energy range is $10-100 \mathrm{keV}$ for ZIP detectors Z2, Z3, Z5 and Z6 and $20-100 \mathrm{keV}$ for detectors Z1 and Z4.

The event rate for single-scatter events in all four germanium detectors is thus tabulated above. This is the neutron population that can lead to WIMP-like nuclear-recoil candidates in the low background data set. For the four germanium detectors, this translates to an expected number of $0.051 \pm 0.024$ events for the duration of Run 118, with all the data efficiencies folded in.

By assuming a production rate obtained from literature based on actual measurements and counter-checked with the output of simulations of the muoninduced neutron production processes in the rock, we arrive at an expected number of $0.051 \pm 0.024$ events in the germanium detectors in Run 118. This number is then compared with the analysis of the veto-anti-coincident nuclear-recoil events 


\begin{tabular}{|c|c|c|c|}
\hline Rates $(\mathrm{cts} / \mathrm{kg} /$ day $)$ & Without effcs & With effcs & Expected Number \\
\hline Coadded Ge & $(2.5 \pm 1.2) \times 10^{-3}$ & $(1.5 \pm 0.5) \times 10^{-3}$ & $0.051 \pm 0.024$ \\
\hline Coadded $\mathrm{Si}$ & $(8.0 \pm 3.8) \times 10^{-3}$ & $(2.4 \pm 1.2) \times 10^{-3}$ & $0.024 \pm 0.011$ \\
\hline
\end{tabular}

Table 8.4: Event rates and expected number of neutrons based on Monte Carlo simulations assuming the spectral representations in Equation 8.1 and Equation 8.2 for the high energy and low energy component of the neutron production in the rock. The rates are in events $/ \mathrm{kg} /$ day. The expected number of neutron candidate events in the four germanium and two silicon detectors for the duration of Run 118 with efficiencies folded in is given in the last row.

in Run 118. Clearly, a one-to-one comparison cannot be made as the data events may include leakage of low-yield electron-recoil events in the signal region, neutrons and possible WIMP candidates. Of course, knowledge of the neutron contribution is essential to our capability of recognizing a WIMP signal. The multiple-scatter events in the veto-anti-coincident data set may include possible neutrons. The next section indicates results of the analysis of the muon veto-anti-coincident data.

\subsection{Analysis of veto-anti-coincident nuclear-recoil events in the data}

Events in the signal region that are not consistent with a trigger in the muon veto and single or multiple-scatter in two or more detectors are possible neutrons, WIMPs or low-yield electrons leaking into the signal region. The possibility of WIMPs multiple-scattering is negligible, so studying the multiple-scatter events provides a means of quantifying the neutron contribution to the WIMP-search data.

In this section, we will examine nuclear-recoil candidates in the un-vetoed population of the low background data. First, we will search for multiple-scatter candidates and subsequently for single-scatter candidates in the data 

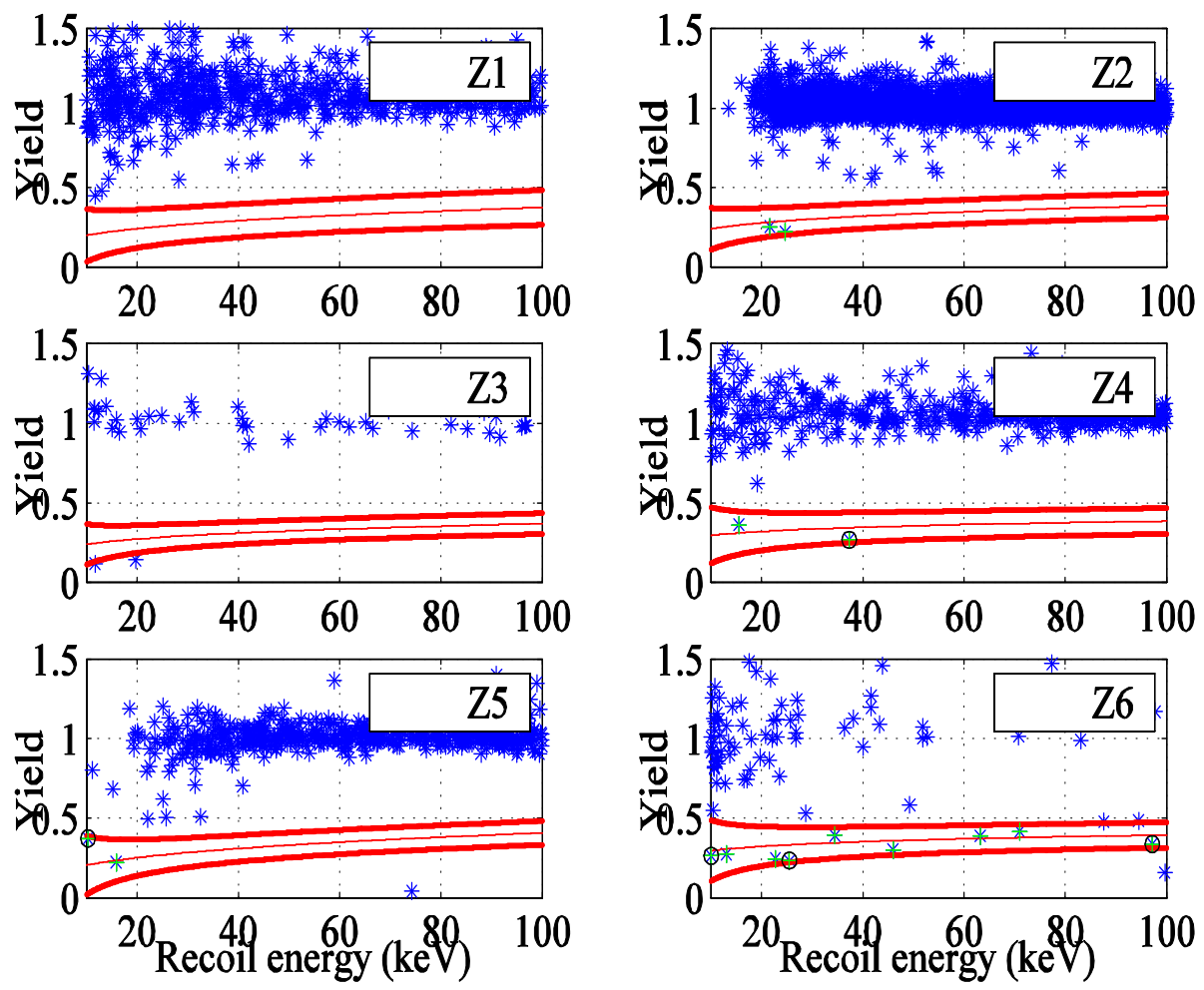

Figure 8.7: Veto anti-coincident multiple scatter nuclear recoil events in the six detectors for the entire low background data set. The signal region is defined within the nuclear recoil bands as shown. The crosses indicate multiple scatter events in the signal region. A rise cut has been applied

\subsubsection{Veto anti-coincident multiple-scatter events}

Figure 8.7 shows the results of the analysis identifying multiple-scatter nuclearrecoil events in the data. There is a rise time cut applied. The circled events are multiple-scatters passing all cuts in one detector and failing the signal region cut in the other detector. No likely nuclear-recoil candidates passed the cuts in this data set.

The above plots show the events in the signal region after applying the rise time cut. The circled events in Figure 8.7 are multiple-scatters passing both 
the nuclear-recoil band and rise time cuts. However, none of these events could be considered as neutron double-scatters as they fail to pass the cuts in the other detector(s). The event in $\mathrm{Z} 4$ is a multiple with an event failing the nuclear-recoil band cut in Z3. The event in Z5 likewise is a multiple-scatter with an event in Z4 which fails the nuclear-recoil band in the second detector. The three events passing all cuts in Z6 also are multiple-scatters with the second scatter being a high-yield event. This indicates that we do not see clear multiple-scatters with both scatters occurring in the nuclear-recoil band. The likelihood of these scatters arising from a gamma scatter in one detector and a low-yield beta in another detector is high considering the possibility of beta leakage due to 'ejectrons'. As was explained in Chapter VII, ejected electrons, or ejectrons as they are called, arise from a gamma recoil in one detector knocking off an electron which scatters on the surface of the neighboring detector. There could also be a likelihood of neutron-gamma multiplescatter events though this has a low probability.

\subsubsection{Veto-anti-coincident single-scatter nuclear-recoil events}

The analysis of veto anti-coincident single-scatter events has already been covered in Chapter VII in reference to the WIMP-search data and the exclusion limits set on the WIMP-nucleon cross-section. As specified earlier, the WIMP-search data was first analyzed blind, i.e., the low background signal region was examined only after all the data analysis cuts had been finalized using the calibration runs with external sources.

However, it was subsequently realized that we had inadvertently been using an inferior pulse fitting algorithm, the F5 pulse fits, meant for saturated pulses for the main analysis. We redid the analysis using the correct pulse fitting algorithm

- the Optimal Filter fits. This caused the signal region to be redefined as well as changed the nature of the events passing the fiducial-volume cuts.

Figures 8.8 shows the WIMP-search data after applying the rise time cuts. The single-scatters in the nuclear-recoil region are identified by circles. This is a 
representation of the blind analysis.

The blind analysis arrived at no candidate events for the single-scatter nuclear-recoil events passing all cuts including the rise time cut. The non-blind analysis had one event in Z5 passing all cuts. This has been documented in Chapter VII. The event, however, is consistent with our estimate of the leakage of lowyield electrons into the signal region and seems likely to be a mis-identified beta. The analysis concluded that we saw no WIMPs or neutrons in the single-scatter population.

\subsection{Predicting the neutron backgrounds}

This chapter summarized our current state of knowledge of the neutron backgrounds at Soudan. Neutrons produced in the shield were simulated using production rates estimated from literature and using GEANT 3.2 simulations to propagate these neutrons from an energy distribution of this incident background. The simulations predicted that we should have seen $1.94 \pm 0.44$ events in the four Germanium detectors which is consistent with the upper limit of 2.3 events set based on the non-observance of such events in the muon coincident data.

The production rates used for the normalization of the output of the simulations were based on the $\mathrm{E}_{\mu}^{0.75}$ dependence of the neutron yield on the mean muon energy at a certain depth. The neutron yield estimated from literature was consistent with the relation of yield with depth as predicted from FLUKA simulations for different materials.

The simulations of the external neutrons also used production rates from literature which were in good agreement with the $\mathrm{E}_{\mu}^{0.75}$ dependence on mean muon energy and relation of yield with depth from FLUKA results. The computer modelling predicted we should have seen $0.051 \pm 0.024$ events in Run 118. 
Veto-anticoincident single scatter nuclear recoils - after rise time cut
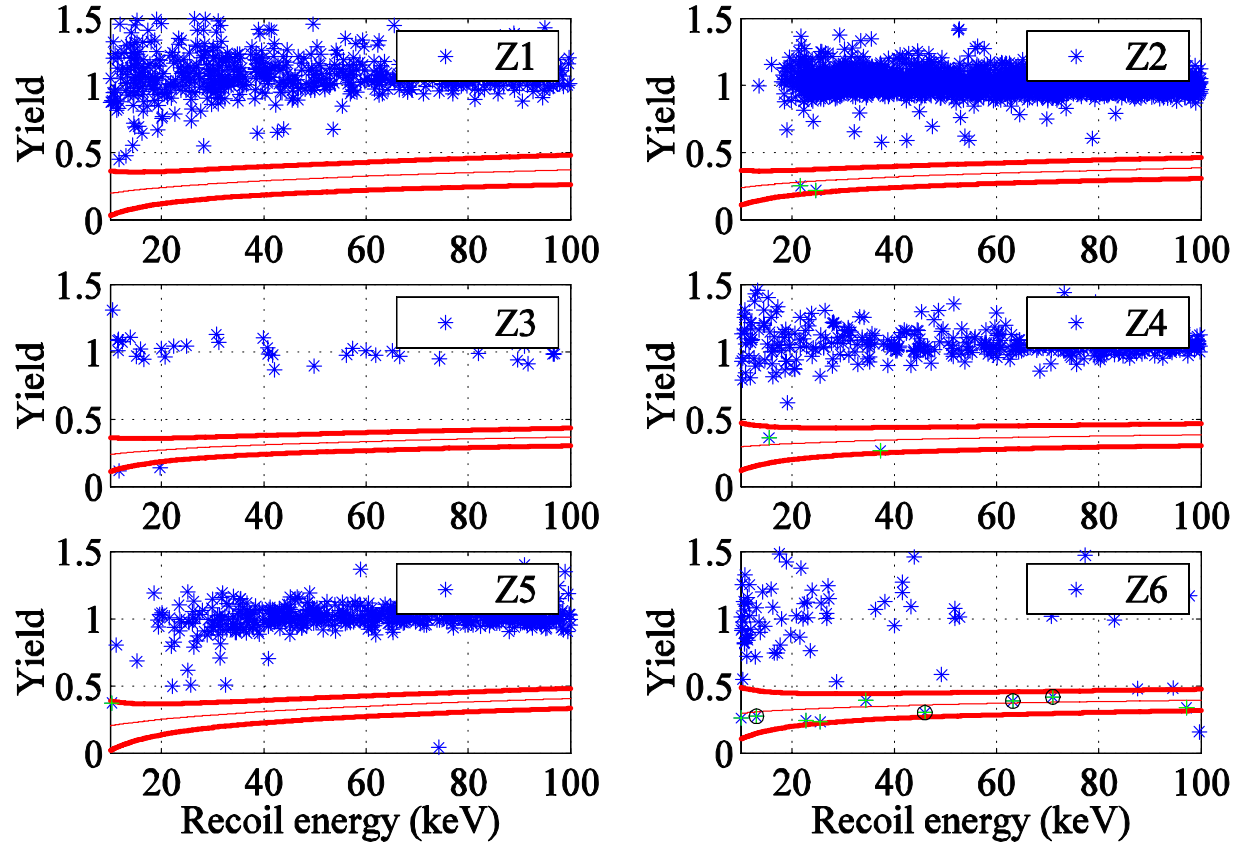

Figure 8.8: Veto anticoincident dataset with the rise time cut applied. The bands indicate the signal region with the green asterix events passing the rise time cut in the signal region. The circled events are single scatters passing all cuts. Only Z6 shows evidence of such events. However, Z6 has a history of ${ }^{14} \mathrm{C}$ contamination which could result in a large surface electron population in the signal region of the detector. This is the blind analysis with the F5 quantities. 
Though the production rates of both the vetoed and un-vetoed neutron populations have the same $\mathrm{E}_{\mu}^{0.75}$ dependence on mean muon energy, the systematic uncertainties associated with the measured production rates of the two data sets as given in the literature do not allow for a reliable prediction of the expected number of punch through neutrons for a more prolonged exposure based on our knowledge of the shield neutrons.

We can, however, use the prediction of event rates from simulations to estimate the number of veto coincident and veto-anti-coincident neutrons we expect to see in the $2500 \mathrm{~kg}$ exposure planned with the full contingent of five towers of six detectors each in the next stage of the CDMS experiment. For the shield neutrons, based on the predicted rates of $(3.6 \pm 0.8) \times 10^{-2}$ evts $/ \mathrm{kg} /$ day, we expect to see

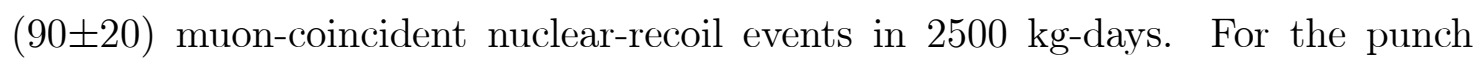
through population, the event rate was determined as $(1.5 \pm 0.5) \times 10^{-3} \mathrm{evts} / \mathrm{kg} /$ day leading to an expectation of $(4 \pm 1)$ neutrons for the same exposure.

With their ability to mimic the dark matter signal in our detectors, the external neutrons are a particularly troublesome background for the CDMS experiment. The short duration of Run 118 coupled with the depth of the new CDMS site ensured that we saw no neutrons in this data set. However, as exposures increase, these neutrons may start to appear in the signal region and may with very large exposures become once again be our limiting background. This is because while gamma and surface electron rates may be considerably higher, the neutrons are the only backgrounds that the detectors do not have the capability of discriminating against and need other means such as simulations of estimating and rejecting them.

Future work on the neutron background will include simulating the production of the neutrons from the muon flux. Simulations will also estimate the effectiveness of the scintillator veto in tracking these neutrons or other particles that are produced and whether there is a need for an additional neutron veto to shield the detectors against such backgrounds. 


\section{References}

[1] K. Ruddick, MINOS Internal Note, NuMI-L-210 (1996).

[2] S. Eichblatt, CDMSNote 9701011 (1997).

[3] S. Yellin, CDMSNote 9704003 (1997).

[4] A. S. Mal'gin et al, JETP Lett., 36, 376 (1983).

[5] L. B. Bezrukov et al, Sov. J. of Nucl. Phys. 17, 51 (1973).

[6] V. A. Kudryavtsev et al, Nucl. Instrum. \& Meth. in Phys. Res. A, 505, 688 (2003).

[7] M. Aglietta et al, Il Nuovo CimentoC, 12, 467 (1989).

[8] Steve Yellin, private communication.

[9] F. F. Khalchukov et al, Il Nuovo Cimento C, 6, 320 (1983).

[10] J. M. Carmona et al, hep-ex/0403009 (2004).

[11] A. da Silva et al, Nucl. Instrum. \&3 Meth. in Phys. Res. A, 354, 553 (1995).

[12] F. F. Khalchukov et al, Il Nuovo Cimento C, 18, 517 (1995).

[13] A. Fasso et al, Proc. of the $4^{\text {th }}$ Intl. Conf. on Calorimetry in high-energy Physics, 493 (1993). 
[14] Y-F. Wang et al, Phys. Rev. D, 64, 013012 (2001).

[15] GEANT Version 3.21.04, GEANT User's Guide, CERN, Geneva (1995).

[16] G. V. Gorshkov et al, Sov J. Nucl. Phys. 12, 187 (1971).

[17] G. V. Gorshkov et al, Sov J. Nucl. Phys. 18, 57 (1974).

[18] G. V. Gorshkov et al, Sov J. Nucl. Phys. 7, 470 (1968).

[19] H. Wulandari et al, hep-ex/0401032.

[20] R. Nelson, B.S. Dissertation, University of California at Santa Barbara (2003), unpublished.

[21] G. V. Gorshkov et al, Sov J. Nucl. Phys. 13, 450 (1971).

[22] O. C. Allkofer and R. D. Andersen, Nucl. Phys. B 8, 402 (1968).

[23] M. A. Meyer et al, Proc. Phys. Soc., 83, 253 (1964).

[24] S. Yellin, private communication. 


\section{Chapter 9}

\section{Towards a Detection}

This thesis described the CDMS search for the elusive dark matter particles called WIMPs. As explained earlier on, the success of a rare-particle search depends greatly on the ability to suppress backgrounds. The move from the 713 meter shallow site at SUF in the first stage of the CDMS WIMP-search to the deep underground site at Soudan, Minnesota was part of the effort to reduce backgrounds, in particular backgrounds arising due to neutrons.

The CDMS experiment plans to scale up to five towers of six detectors each for a total of $1200 \mathrm{~kg}$-days exposure. Two additional towers will be installed in the CDMS III phase of the experiment scaling the exposure to $3400 \mathrm{~kg}$-days by the end of 2007. The current data run saw no neutron backgrounds in part because of the relatively short duration of the run. As exposures increase, such backgrounds will once again be a source of concern. The experiment plans to tackle this by implementing new shielding schemes. Some of them include having an additional layer of scintillator to allow for the muon veto to better track external neutrons incident on the shield. This is expected to improve the neutron rejection by a factor of 2 -3 over the present case. As external neutrons produced by muons interacting in the rock are also accompanied by hadronic showers. Devising a means of tracking down the showers will allow us to discriminate against this limiting background. 
Better background reduction and rejection techniques and greater exposures are now allowing dark matter searches to explore greater region of WIMP parameter space and aim for sensitivities of the order of $10^{-9}-10^{-10} \mathrm{pb}$ to the WIMP-nucleon cross section for WIMP masses of $40-80 \mathrm{GeV} / \mathrm{c}^{2}$. The advances in the detection techniques allow us to be hopeful of a possible WIMP detection. Coupled with the accelerator searches at the upcoming Large Hadron Collider, this holds promise for defining new physics as well as a better understanding of the composition of the Universe. 


\section{Bibliography}

[1] D. Abrams et al., Phys. Rev. D, 66, 122003 (2002)

[2] R. Abusaidi et al, Phys. Rev Lett., 84, 5695 (2000).

[3] M. Aglietta et al, Il Nuovo CimentoC, 12, 467 (1989).

[4] S. Agostinelli et al, Nucl. Instrum. 83 Meth. in Phys. Res. A, 506, 250 (2003).

[5] J. Ahrens et al., Phys. Rev. D 66, 032006 (2002).

[6] D. S. Akerib et al., Phys. Rev. D, 68, 082002 (2003).

[7] D. S. Akerib et al., astro-ph/0405033 (2004).

[8] D. S. Akerib et al, Nucl. Instrum. 63 Meth. in Phys. Res. A, 520, 116 (2004).

[9] O. C. Allkofer and R. D. Andersen, Nucl. Phys. B 8, 402 (1968).

[10] P. Antonioli et al., Astropart. Phys., 7, 357 (1997).

[11] http://www.astrographics.com/GalleryPrintsIndex/GP0017.html.

[12] E. J. Axton and A. G. Bardell, Metrologia, 21, 59 (1985).

[13] N. Bahcall et al, Astrophys. J., 447, 81 (1995).

[14] J. C. Barton, Proceedings of the 19th International Cosmic Ray Conference, 8, 98 (1985). 
[15] S. W. Barwick et al., Phys. Rev. Lett. 75, 390 (1995).

[16] E. Battaner and E. Florido, Fund. Cosmic Physics, 21, 1 (2000).

[17] D. Bauer, CDMS Internal Note (2001).

[18] C. L. Bennett, M. Halpern et al, Astrophys. J. Suppl. 148, 1 (2003).

[19] A. Benoit et al., Phys. Lett. B 545, 43 (2002).

[20] V. S. Berezinsky, A. Bottino and V. de Alfaro, Phys. Lett. B 274, 122 (1992).

[21] L. Bergstrom, J. Edsjo and P. Ullio, Astropart.Phys. 9, 137 (1998).

[22] R. Bernabei et al., Phys. Lett. B 480, 23 (2000).

[23] L. B. Bezrukov et al., Sov. J. Nucl. Phys., 17, 51 (1973).

[24] S. Burles et al. Astrophys. J., 483, 778 (1997).

[25] B Cabrera, Proc. of the VIII Intl. Workshop on Low Temp. Detectors, Dalfsen, The Netherlands (1999).

[26] R.G. Carlberg et al., Astrophys. J., 462, 32 (1996).

[27] J. M. Carmona et al, hep-ex/0403009 (2004).

[28] J. Carson et al, hep-ex/0404042 (2004).

[29] Castagnoli, C. et al., Astropart. Phys., 6, 187 (1997).

[30] G. Chardin and G. Gerbier, Proc. of the IV Internat. Workshop on Identification of Dark Matter, 470 (2002).

[31] V. Chazal et al, Nucl. Instrum. ES Meth. in Phys. Res. A, 490, 334 (2002).

[32] M. Chen et al, Nucl. Instrum. \&3 Meth. in Phys. Res. A, 336, 232 (1993).

[33] E. Corbelli and P. Salucci, MNRAS, 311, 441 (2000). 
[34] A. da Silva, Ph. D. Thesis, The University of British Columbia, (1996).

[35] Davis, D. S., and White, R. E., III., Astrophys. J., 470, 35 (1996).

[36] D. Day and L. Baudis, CDMS Run 118 Ebook note 108 (2004).

[37] D. D. Driscoll, Ph.D. Thesis, Case Western Reserve University (2004).

[38] D.D. Driscoll, Run 21 Ebook Note 305 (2003).

[39] S. Eichblatt, CDMSNote 9701011 (1997).

[40] O. Elgaroy et al, Phys. Rev. Lett., 89061301 (2002).

[41] J. R. Ellis et al, Nucl. Phys. B, 238, 453 (1984).

[42] A.E. Evrard et al, Astrophys. J., 469, 494507 (1996).

[43] A. C. Fabian and S. W. Allen, Proc. of the XXI Texas Symposium on Relativistic Astrophysics, Dec. 9-13 (2002).

[44] A. Fasso et al, Proc. of the $4^{\text {th }}$ Intl. Conf. on Calorimetry in high-energy Physics, 493 (1993).

[45] J. Feng, hep-ph/0405215 (2004).

[46] H. C. Fesefeldt III, Gheisha version 7.03 (1985).

[47] T. Florkowski et al, Nuclear Geophysics, 2, 1 (1988).

[48] GEANT Version 3.21.04, GEANT User's Guide, CERN, Geneva (1995).

[49] H. Goldberg, Phys. Rev. Letts., 50, 1419 (1983).

[50] S. R. Golwala, Ph.D. Thesis, University of California at Berkeley (2000), unpublished.

[51] M. W. Goodman and E. Witten, Phys. Rev. D 31, 3059 (1985). 
[52] G. V. Gorshkov et al., Sov. J. Nucl. Phys., 18, 57 (1974).

[53] G. V. Gorshkov et al., Sov. J. Nucl. Phys., 7, 470 (1968).

[54] G. V. Gorshkov et al., Sov. J. Nucl. Phys., 12, 187 (1971).

[55] G. V. Gorshkov et al., Sov. J. Nucl. Phys., 13, 450 (1971).

[56] Gurentsov, V.I. et al., Sov. J. Nucl. Phys., 23, 527 (1976).

[57] N.W. Halverson et al., Astrophys. J., 568, 38 (2002).

[58] A. Habig et al., hep-ex/0106024.

[59] S. Hart, Nucl. Phys. B Proc. Suppl. 110, 91 (2002).

[60] Heisinger, B., Ph.D. Thesis, Technical University of Munich (1998), unpublished.

[61] G. Heusser, Ann. Rev. Nucl. Part. Sci. 45, 543 (1995).

[62] G. Huey, R. H. Cyburt and B. D. Wandelt, astro-ph/0307080 (2003).

[63] K. D. Irwin, Ph.D. Thesis, Department of Physics, Stanford University (1995).

[64] K.D. Irwin, Appl. Phys. Lett., 66, 1998 (1995).

[65] K.D. Irwin et al, Rev. Sci. Instrum., 66, 10 (1995).

[66] J. O. Johnson and T. A. Gabriel, MICAP: A Monte Carlo Ionization Chamber Analysis Package (1993).

[67] G. Jungman et al, Physics Reports, 267, 195373 (1996).

[68] F. F. Khalchukov et al, Il Nuovo Cimento C, 6, 320 (1983).

[69] F. F. Khalchukov et al, Il Nuovo Cimento C, 18, 517 (1995). 
[70] G.F. Knoll, Radiation Detection and Measurement., John Wiley and Sons, Inc., (1999).

[71] V. A. Kudryavtsev et al, Nucl. Instrum. E Meth. in Phys. Res. A, 505, 688 (2003).

[72] D. Lal and B. Peters, Handbuchder Physik, 46/2, 551 (1967).

[73] B.W. Lee and S. Weinberg, Phys. Rev. Letts., 39, 165 (1977).

[74] J.D. Lewin and P. F. Smith, 6Astropart. Phys., 6, 87 (1996).

[75] M. Loewenstein and R. E. White, Astrophys. J., 518, 50 (1999).

[76] P. Luke, J. Appl. Phys., 64, 6858 (1988).

[77] B. Macdonald et al. Phys. Rev.B, 139, 5 (1965).

[78] A. S. Mal'gin et al, JETP Lett., 36, 376 (1983).

[79] V. Mandic, CDMS Run 21 Ebook Note 191.

[80] V. Mandic et al., Nucl. Instr. Meth. A 520, 171 (2004).

[81] V. Mandic, Ph.D. Thesis, University of California at Berkeley (2004), unpublished.

[82] M. A. Meyer et al, Proc. Phys. Soc., 83, 253 (1964).

[83] B. Neganov and V. Trofimov, Pis'ma v Zhurnal Experimental'noi $i$ Teoreticheskoi Fiziki, 28, 356 (1978).

[84] R. Nelson, B.S. Dissertation, University of California at Santa Barbara (2003), unpublished.

[85] C.B. Netterfield et al., Astrophys.J., 571, 604 (2002).

[86] J. H. Oort, Bull. Astron. Inst. Neth. 6, 249 (1932). 
[87] J. P. Ostriker and P. J. E. Peebles, Ap. J., 186, 467 (1973).

[88] PAW Version 2.13/08, Physics Analysis Workstation Reference Manual, CERN, Geneva (2002).

[89] J. A. Peacock, Cosmological Physics, Cambridge University Press (1999).

[90] P. J. E. Peebles, Principles of Physical Cosmology. Princeton University Press (1993).

[91] T. A. Perera, Ph.D. Thesis, Case Western Reserve University (2002), unpublished.

[92] M. Persic, P. Salucci, and F. Stel, Mon. Not. Roy. Astron. Soc. 281, 27 (1996).

[93] http://wswww.physik.uni-mainz.de/zeitnitz/gcalor/gcalor.html

[94] http://pupgg.princeton.edu/ martin/Ross_Nathaniel_JP.pdf

[95] W. Rau, CDMSNote 0206004 (2003).

[96] R. C. Reedy, J. R. Arnold and D. Lal, Ann. Rev. Nucl. Part. Sci., 33, 505 (1983).

[97] V. C. Rubin and W. K. Ford, Ap. J., 159, 379 (1970).

[98] K. Ruddick, MINOS Internal Note, NuMI-L-210 (1996).

[99] O. G. Ryajskaya and G. T. Zatsepin, Proc. of the IX Internat. Conf. on Cosmic Rays, London, 3, 987 (1966).

[100] T. Saab, Ph.D. thesis, Stanford University (2002).

[101] J. Sander, CDMS Run 118 Ebook note 226 (2004).

[102] R. Schnee, Run 21 Ebook Note 275 (2003).

[103] W. U. Schröder, Ph.D. Thesis, Technische Hochschule Darmstadt (1971). 
[104] E. Segre, Nuclei and Particles, Second Edition W A Benjamin Inc. (1977).

[105] E. P. S. Shellard and R. A. Battye, astro-ph/9802216.

[106] T. Shutt et al., Nucl. Instr. and Meth. A, 444, 340344 (2000).

[107] P. Singer, Springer Tracts in Modern Physics, 71, 39 (1974).

[108] I. Smail et al. Astrophys. J., 479, 70 (1996).

[109] S. Smith, Astrophys J., 83, 23 (1936)

[110] D. N. Spergel et al, Astrophys.J.Suppl., 148, 175 (2003).

[111] D.H. Stoddard and H.E. Hootman, ${ }^{252}$ Cf Shielding Guide, Savannah River Laboratory Internal Report, DP-1245 (1971).

[112] R. Stompor et al., Astrophys. J., 561, 7 (2002).

[113] Table of Radioactive Isotopes, http://nucleardata.nuclear.lu.se

[114] D. Tytler et al., Physica Scripta, T85, 1231 (2000).

[115] T. Van Duzer, Principles of Superconducting Devices and Circuits, PrenticeHall, New Jersey, 1999.

[116] H. Wulandari et al, hep-ex/0401032 (2004).

[117] H. Wulandari, Ph.D. Thesis, Technical University of Munich (2003).

[118] G. Wang and S. Kamat, CDMS Run 118 Ebook note 125 (2004).

[119] Y-F. Wang et al, Phys. Rev. D, 64, 013012 (2001).

[120] S. Yellin, CDMSNote 9704003 (1997).

[121] F. Zwicky, Helv. Phys. Acta, 6, 124 (1933). 